\title{
Geophysical Pattern Formation of Salt Playa
}

\author{
for the award of the degree \\ "Doctor rerum naturalium" (Dr. rer. nat.) \\ Division of Mathematics and Natural Sciences \\ of the Georg-August-Universität Göttingen
}

submitted by

Jana LASSER

from Graz, Austria

Göttingen, March 7, 2019 



\section{Members of the Thesis Committee}

Lucas Goehring (reviewer)

School of Science and Technology, Nottingham Trent University

Andreas Tilgner (reviewer)

Geophysical fluid dynamics, Georg-August-Universität Göttingen

\section{Eberhard Bodenschatz}

Fluid physics, pattern formation and biocomplexity, Max Planck Institute for Dynamics and Self-Organization Göttingen

Date of the oral examination: February $12^{\text {th }}, 2019$ 



\section{Declaration of Authorship}

I, Jana LASSER, declare that this thesis titled, "Geophysical Pattern Formation of Salt Playa" and the work presented in it are my own. I confirm that:

- This work was done wholly or mainly while in candidature for a research degree at this University.

- Where any part of this thesis has previously been submitted for a degree or any other qualification at this University or any other institution, this has been clearly stated.

- Where I have consulted the published work of others, this is always clearly attributed.

- Where I have quoted from the work of others, the source is always given. With the exception of such quotations, this thesis is entirely my own work.

- I have acknowledged all main sources of help.

- Where the thesis is based on work done by myself jointly with others, I have made clear exactly what was done by others and what I have contributed myself.

Signed:

Date: 

"Mathematics began to seem too much like puzzle solving. Physics is puzzle solving, too, but of puzzles created by nature, not by the mind of man. "

Maria Göppert-Mayer 



\title{
Abstract
}

\author{
Jana LASSER
}

Geophysical Pattern Formation of Salt Playa

Patterns, such as the hexagonal salt ridges that emerge in salt deserts around the world, are a common phenomenon in geophysical settings. They are similar to other natural phenomena such as fairy circles, columnar joints and patterned ground, whose origin can be explained by an instability in a dynamical system. So far, the origin of polygonal salt patterns in salt deserts is unknown, even though they are a common landform and their understanding makes an important contribution to climate modeling and the understanding of the emission of atmospheric dust from their surface. In this work I provide a model that explains the emergence of the salt ridge hexagons. I present evidence, which confirms the predictions made by my model. To explain the driving mechanism of salt polygons, I couple the surface expression of ridges on the salt crust to the dynamics in the underground and model the process as the buoyancy driven convection of the saline water in the porous medium below the salt crust. I investigate the driving mechanism by means of mathematical modeling, numerical simulation, analogue experiments and a field study. I find the conditions for instability of the stratified saline water and present direct hydrological evidence for the existence of plumes of high salinity which are co-located with the patterns visible at the surface. With my model I am able to explain the length - and timescale of pattern emergence as well as the robustness of pattern wavelengths against changing natural circumstances. The results from my investigation of buoyancy driven convection in porous media can be connected to other research of similar systems, such as $\mathrm{CO}_{2}$ sequestration. Last but not least, with my work I hope to make a contribution to the better understanding of these otherworldly landscapes that draw the eye and fascinate the observer, in the hope that a better understanding of nature will lead to increased efforts to preserve it. 



\section{Acknowledgements}

I thank my supervisor Lucas Goehring for the possibilities and advice he gave me throughout the work on my project and the freedom to pursue my own ideas. I would also like to thank Stephan Herminghaus for giving my research project a home in his department and supporting me in many ways.

I want to thank the Georg August University Göttingen, the Max Planck Society and the Göttingen Graduate school for Neurosciences, Biophysics and Molecular Biosciences for the funding and learning opportunities they gave me.

I would like to thank my colleagues at the MPI for Dynamics and Self-Organization: Fabian, Felix, Jéremy and Chamkor for many good scientific discussions and lots of fun over coffee. I also want to thank Kris, Markus, Wolf, Monika and Thomas for the administrative and technical support.

I want to thank my Thesis Advisory Committee members Andreas Tilgner and Eberhard Bodenschatz and acknowledge the scientific input to my work they gave me in the yearly meetings. I would also like to thank the members of my defense committee: Karen Alim, Volker Karius and Andreas Dillmann.

I want to thank Jo Nield from the University of Southampton for her support during the two field campaigns and her contributions to the data collection and analysis with the terrestrial laser scanner. I want to thank Grace Holder from the Los Angeles Department for Land ans Water, for ground support and valuable information at Owens Lake. I would also like to thank Maike Lorenz from the Göttingen algae collection for giving the algae I sampled a new home and providing me with stunning pictures of the microorganisms, inhabitating Owens Lake.

I want to thank Birte Thiede and Marcel Ernst, whom I had the pleasure to work with during their Bachelor's and Master's thesis respectively. Birte made the 3-D proof-of-principle experiments possible, whereas without Marcel's work on the theory and numerical simulations, a large part of my work would not have been possible.

Last but not least, I would like to thank my colleagues and friends from the PhDnet: Angela, Maria, Greta, Julia, Mohamed, Severin, Leo, Gabe, Lisa, Rafa, Teresa, Filippo. They gave me motivation when my science did not motivate me and worked with me on many projects outside the small microcosm of sand, salt and water that my project was. 



\section{Contents}

Declaration of Authorship $\quad$ i

Abstract $\quad$ v

Acknowledgements vii

1 Introduction $\quad 1$

1.1 Salt deserts: a common landform . . . . . . . . . . . . . . . . . 3

1.2 Importance of salt pans for climate and human livelihood . . . . . . . . 4

1.2.1 Climate-surface interactions . . . . . . . . . . . . . . . 4

1.2.2 Human-surface interactions . . . . . . . . . . . . . . 5

Owens Lake history $\ldots \ldots \ldots \ldots$

Toxic dust . . . . . . . . . . . . . . 6

Surface management . . . . . . . . . . . . 6

1.2.3 Aesthetics and human curiosity . . . . . . . . . . . . . . . 9

1.3 Salt polygons as instability in a dynamical system . . . . . . . . . . . 10

1.3.1 From description to model . . . . . . . . . . . . . 11

1.3 .2 Existing approaches . . . . . . . . . . . . . . . 12

1.4 Predictions . . . . . . . . . . . . . . . . . . . . . . 14

1.5 Summary . . . . . . . . . . . . . . . . . . . . 15

1.6 Scope of this thesis . . . . . . . . . . . . . 15

2 Theory 17

2.1 Momentum balance of fluid flow . . . . . . . . . . . . . . . . 17

2.2 Flow in a porous medium . . . . . . . . . . . . . 18

2.3 Coupling to a dissolved quantity . . . . . . . . . . . . . . . 19

2.4 Coupled equations for fluid and salt transport in a porous medium . . 21

2.5 Non-dimensionalization . . . . . . . . . . . . . . . . . 21

2.6 Boundary Conditions . . . . . . . . . . . . . . . . . 23

2.6 .1 Top boundary . . . . . . . . . . . . . . . . . . . . . 24

2.6.2 Steady state of the salinity distribution . . . . . . . . . . . . . 25

2.6 .3 Other boundaries . . . . . . . . . . . . . . . . . . . 25

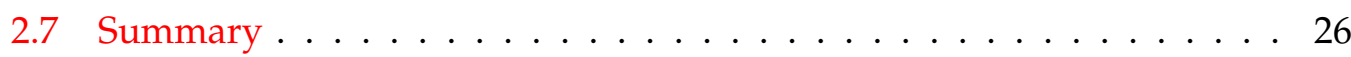


3 Materials and Methods $\quad 27$

3.1 Simulation . . . . . . . . . . . . . . . . . . 28

3.1 .1 Numerical Approach . . . . . . . . . . . . . . . . . . . . . 29

3.1 .2 Dimensionality . . . . . . . . . . . . . . . 30

3.1 .3 Differential Equations . . . . . . . . . . . . . . 30

3.1.4 Compact Finite Difference Scheme . . . . . . . . . . . . . . . . 31

3.1 .5 Pseudo-Spectral Approach . . . . . . . . . . . . 33

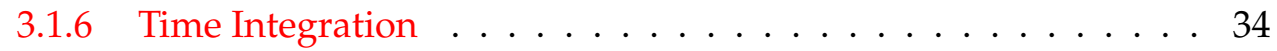

3.1 .7 Simulation Parameters . . . . . . . . . . . . . . . . . . 34

System size, resolution, duration . . . . . . . . . . . 34

Rayleigh Number . . . . . . . . . . . . . . . . . 36

Evaporation modulation wavelength and amplitude . . . . 36

Initial conditions . . . . . . . . . . . . . . . 36

Validation of growth rates . . . . . . . . . . . 37

3.1 .8 Measurements . . . . . . . . . . . . . . . . . 38

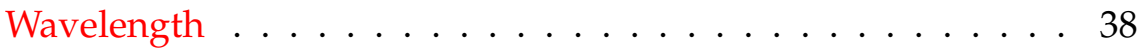

Plume alignment . . . . . . . . . . . 38

Velocity scaling . . . . . . . . . . . . . . . 39

3.1 .9 Summary . . . . . . . . . . . . . . . . . . . 39

3.2 Experiment. . . . . . . . . . . . . . . . . . 39

3.2.1 Two dimensional Hele-Shaw Setup . . . . . . . . . . . . . . 40

Experiment Duration . . . . . . . . . . . . . . . . . 42

Flow Visualization . . . . . . . . . . . . . . . . 43

Wavelength measurements . . . . . . . . . . . . . . 44

3D Setup . . . . . . . . . . . . . . . . . . 45

3.2 .2 Sand characteristics . . . . . . . . . . . . . 46

Grain size distribution . . . . . . . . . . . . . . . . . . 46

Sauter diameter . . . . . . . . . . . . . . . 4 47

Permeability . . . . . . . . . . . . . . . 48

Porosity . . . . . . . . . . . . . . . . 49

3.2.3 Evaporation Rate . . . . . . . . . . . . . . . . . . . . 50

Controlling evaporation rate . . . . . . . . . . . . 50

Measuring evaporation rate . . . . . . . . . . . . 51

3.2.4 Salt Concentration . . . . . . . . . . . . . . . . 53

Controlling salt concentration in the experiment . . . . . . 53

Measuring salt concentration in the experiment and the field . . 54

3.2 .5 Summary . . . . . . . . . . . . . . . . . 56

3.3 Field Research . . . . . . . . . . . . . . . . . . . . . 56

3.3.1 Subsurface sampling . . . . . . . . . . . . . 58

Coring . . . . . . . . . . . . . . . . 5 59

Trenching . . . . . . . . . . . . . . . 59

Salt concentration measurements . . . . . . . . . . . 60 
Pore water extraction . . . . . . . . . . . . . . . . 61

3.3.2 Pattern length scale characterization . . . . . . . . . . . . . 61

Terrestrial laser surface scans . . . . . . . . . . . . . . . 61

TLS Data processing . . . . . . . . . . . . . . . . 62

Crust thickness and ridge height . . . . . . . . . . . . . . . 63

Timelapse imaging . . . . . . . . . . . . . . . . 63

3.3.3 Salt crust characterization . . . . . . . . . . . . . . . . 64

X-ray diffraction analysis of crust minerals . . . . . . . . 65

Spectrometry analysis of crust minerals . . . . . . . . . . . 66

Biological sample components . . . . . . . . . . . 66

3.3 .4 System parameters . . . . . . . . . . . . . . . . . . . 68

Permeability . . . . . . . . . . . . . . 68

Evaporation rate . . . . . . . . . . . . . 68

Weather at the field sites . . . . . . . . . . . . . . 69

3.3 .5 Field site geology . . . . . . . . . . . . . . . 70

Owens Lake geology . . . . . . . . . . . . . . . . 70

Badwater Basin geology . . . . . . . . . . . . . 70

Bristol Playa and Soda Dry Lake . . . . . . . . . . . . 72

3.3 .6 Summary ........................ . . 72

$\begin{array}{lll}4 & \text { Results } & \mathbf{7 5}\end{array}$

4.1 Introduction . . . . . . . . . . . . . . . . . 75

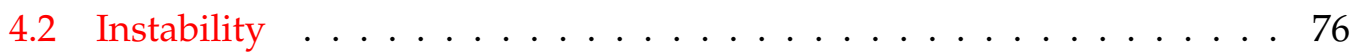

4.2.1 Linear stability analysis . . . . . . . . . . . . . 76

4.2 .2 Instability under field conditions . . . . . . . . . . . 78

Site nomenclature . . . . . . . . . . . . . . . 79

Grain size, permeability and porosity . . . . . . . . . . . . . 80

Salinity gradient . . . . . . . . . . . . . . 82

Field Rayleigh numbers . . . . . . . . . . . . . . . 82

4.2 .3 Instability under laboratory conditions . . . . . . . . . . 83

4.2 .4 Summary . . . . . . . . . . . . . . . . . . . 84

4.3 Density stratification . . . . . . . . . . . . . . . 84

4.3 .1 System age . . . . . . . . . . . . . . . . 85

4.3.2 Properties of the salinity gradient . . . . . . . . . 86

4.3.3 Salinity gradients in the experiment . . . . . . . . . . . 87

4.3 .4 Salinity gradients in the field . . . . . . . . . . . . . 88

Effective diffusivity in the field . . . . . . . . . . . . . . 88

Salinity gradient length scale . . . . . . . . . . . . . . 89

4.3 .5 Summary . . . . . . . . . . . . . . . . . . . 92

4.4 Convection . . . . . . . . . . . . . . . . . 93

4.4.1 Convection in experiments . . . . . . . . . . . . 93

Flow dynamics . . . . . . . . . . . . . . . . . 93 
Salt concentration distribution . . . . . . . . . . . . 94

3D convection . . . . . . . . . . . . . . . . . 95

4.4 .2 Convection in the field . . . . . . . . . . . . . . . . . 96

Reliability of field results . . . . . . . . . . . . . 97

Salinity profiles of trench sites . . . . . . . . . . . . 97

Statistical significance . . . . . . . . . . . . . . 999

4.4 .3 Summary . . . . . . . . . . . . . . . . . 100

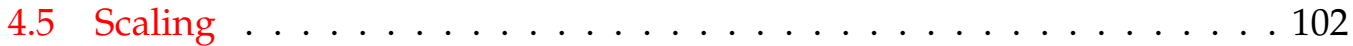

4.5.1 Scaling of wavenumber with Rayleigh number . . . . . . . . . . 102

Qualitative coarsening . . . . . . . . . . . . . . . 103

Quantifying coarsening in simulations . . . . . . . . . . . . . 104

4.5.2 Wavenumbers in experiments . . . . . . . . . . . . 106

4.5.3 Pattern length scales in the field . . . . . . . . . . . . . . . 107

4.5 .4 Summary . . . . . . . . . . . . . . . . . . . . . 109

4.6 Feedback . . . . . . . . . . . . . . . . . . . . . . . . . 112

4.6.1 Non-uniform boundary condition . . . . . . . . . . . . 112

4.6 .2 Pinning . . . . . . . . . . . . . . . . . . . . . 113

4.6 .3 Feedback in the field . . . . . . . . . . . . . . . . . 115

4.6 .4 Summary . . . . . . . . . . . . . . . . . . . 116

4.7 Ridge growth . . . . . . . . . . . . . . . . . . 117

4.7.1 Indirect evidence for ridge growth . . . . . . . . . . . . . . 117

4.7.2 Direct evidence for ridge growth . . . . . . . . . . . . 118

4.7 .3 Summary . . . . . . . . . . . . . . . . . . . . . 120

4.8 Conclusion . . . . . . . . . . . . . . . . . . . . . . 121

5 Discussion $\quad 125$

5.1 Approximations . . . . . . . . . . . . . . . . . . 125

5.1 Diffusion and Dispersion . . . . . . . . . . . . . 125

5.1.2 Dynamic viscosity and evaporation rate . . . . . . . . . . . . 126

5.2 Simulation . . . . . . . . . . . . . . . . . . . 127

5.2.1 Comparison to literature . . . . . . . . . . . . . . . . . . 127

5.2.2 Limitations of the simulation . . . . . . . . . . . . . . . 129

5.3 Experiments . . . . . . . . . . . . . . . . . . . 130

5.3 .1 Cell dimensions . . . . . . . . . . . . . . . . . . . . 130

5.3 .2 Direct observation of crust growth . . . . . . . . . . . . . 131

5.4 Field results . . . . . . . . . . . . . . . . . . . 132

5.4.1 Instability and salinity profiles . . . . . . . . . . . . . 133

5.4.2 Redundant samples and measurements . . . . . . . . . . . . 134

5.4 .3 Salt crusts . . . . . . . . . . . . . . . . . . . . . 137

5.4.4 Biological sample content . . . . . . . . . . . . . . 138

5.5 Why hexagons? . . . . . . . . . . . . . . . . . . . . 138

5.6 Summary . . . . . . . . . . . . . . . . . . . . . . . 139 
6 Conclusion 141

6.1 Summary . . . . . . . . . . . . . . . . . . . . . . . . . 141

6.1 .1 Instability . . . . . . . . . . . . . . . . . 141

6.1 .2 Density stratification . . . . . . . . . . . . . . . . . 142

6.1 .3 Convection . . . . . . . . . . . . . . . . . . . 142

6.1 .4 Scaling . . . . . . . . . . . . . . . . . 143

6.1 .5 Feedback . . . . . . . . . . . . . . . . . . . 143

6.1 .6 Concluding remarks . . . . . . . . . . . . . . . . . . . 144

6.2 Outlook . . . . . . . . . . . . . . . . . . . . . . . . . . . 144

6.2 .1 Simulations . . . . . . . . . . . . . . . . . . . . 144

6.2 .2 Experiment . . . . . . . . . . . . . . . . . 147

6.2 .3 Field . . . . . . . . . . . . . . . . . . . 148

$\begin{array}{ll}\text { A Field site descriptions } & 151\end{array}$

A.1 Field site locations . . . . . . . . . . . . . . . . . . . . . . . . . 151

A.2 Site description Badwater Basin (1) . . . . . . . . . . . . . . 152

A.3 Site description Badwater Basin (2) . . . . . . . . . . . . . . . . . 153

A.4 Site description Badwater Basin (3) . . . . . . . . . . . . . . . . 154

A.5 Site description Badwater Basin (4) . . . . . . . . . . . . . . . . . 155

A.6 Site description Badwater Basin (5) . . . . . . . . . . . . . . . 156

A.7 Site description Bristol Playa . . . . . . . . . . . . . . . . . . . 157

A.8 Site description Owens Lake T10-3 . . . . . . . . . . . . . . . . 158

A.9 Site description Owens Lake T16 . . . . . . . . . . . . . . . . . . 159

A.10 Site description Owens Lake T2-4 . . . . . . . . . . . . . . . . . . . 160

A.11 Site description Owens Lake T2-5 (1) . . . . . . . . . . . . . . . . . 161

A.12 Site description Owens Lake T2-5 (2) . . . . . . . . . . . . . . . . 162

A.13 Site description Owens Lake T2-5 (3) . . . . . . . . . . . . . . . . . 163

A.14 Site description Owens Lake T25-3 (1) . . . . . . . . . . . . . . . . . 164

A.15 Site description Owens Lake T25-3 (2) . . . . . . . . . . . . . . . . . 165

A.16 Site description Owens Lake T27-A (1) . . . . . . . . . . . . . . 166

A.17 Site description Owens Lake T27-A (2) . . . . . . . . . . . . . . . 167

A.18 Site description Owens Lake T27-A (3) . . . . . . . . . . . . . . 168

A.19 Site description Owens Lake T27-S . . . . . . . . . . . . . . . . . . 169

A.20 Site description Owens Lake T29-3 (1) . . . . . . . . . . . . . . . . . 170

A.21 Site description Owens Lake T29-3 (2) . . . . . . . . . . . . . . . . . 171

A.22 Site description Owens Lake T32-1-L1 (1) . . . . . . . . . . . . . . . 172

A.23 Site description Owens Lake T32-1-L1 (2) . . . . . . . . . . . . . . . . 173

A.24 Site description Owens Lake T32-1-L1 (3) . . . . . . . . . . . . . . . . 174

A.25 Site description Owens Lake T36-3 (1) . . . . . . . . . . . . . . . . . 175

A.26 Site description Owens Lake T36-3 (2) . . . . . . . . . . . . . . 176

A.27 Site description Owens Lake T36-3 (3) . . . . . . . . . . . . . . . . 177

A.28 Site description Owens Lake T8-W . . . . . . . . . . . . . . 178 
xiv

$\begin{array}{lr}\text { B Mineral quantification } & \mathbf{1 7 9}\end{array}$

B.1 Pore water minerals . . . . . . . . . . . . . . . . . . . . 179

B.2 Crust minerals . . . . . . . . . . . . . . . . . . . . . 180

B.3 Quantification of dissolved ions . . . . . . . . . . . . . . . . 181

$\begin{array}{ll}\text { C Owens Lake microbiology } & 183\end{array}$

$\begin{array}{ll}\text { Bibliography } & 185\end{array}$ 


\section{List of Figures}

1.1 Schematic representation of salt playa as a geographical sink. . . . . . 1

1.2 Exemplary salt crust patterns found in salt pans at the west coast of the US. Panels (a), (b) and (c) show fingers, squares and small ridges respectively. Panels (d), (e) and (f) show a dome, small bumps and winding precipitation patterns respectively. Dollar coins were placed in the images for scale. . . . . . . . . . . . . . . . . . .

1.3 Salt polygons found in several salt deserts all over the world: (a) Badwater Basin in the Death Valley, California, USA, (b) Salar de Uyuni, Chile, (c) Namak Lake, Iran and (d) the Dead Sea, Israel, image courtesy Lucas Goehring. Polygons visible in the photographs have a diameter of $1-2$ meters. . . . . . . . . . . . . . . . . 3

1.4 Implementation of the dust management project (source: Google Earth). 5

1.5 The surface of Owens Lake is heavily managed to prevent dust emission by (a) shallow flooding, (b) plantation of salt resistant vegetation, (c) coverage with gravel, (d) flooding with brine and subsequent formation of an artificial and more stable salt crust and (e) trenching. (f) shows the sharp transition between a cell managed with vegetation and a brine pool and (g) shows the polygonal structures emerging from a brine pool. The red color is due to a bloom of the algae Dunaliella Salina, which finds a natural habitat in the salt brine. Images (e), (f) and (g) courtesy David Meisel. . . . . . . . . . . . . .

1.6 Exemplary polygons from Owens Lake. (a) and (b) polygons and continuous crust at an older managed site. (c) polygons at an unmanaged site near the east shore of the lake. (d) tepee-like ridge at a recently flooded site. The handle of the rock hammer shown for scale has a length of $31 \mathrm{~cm} \ldots \ldots \ldots \ldots \ldots \ldots \ldots$

1.7 Image from the Movie Star Wars: the Last Jedi [39], featuring the planet "Crait" with its otherworldly looking landscape. The movie was shot in 2017, the depicted scene is inspired the Salar de Uyuni, Chile. The iconic salt polygons of the Salar are visible in the bottom half of the

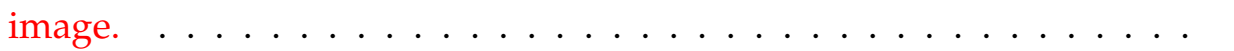


1.8 Examples of patterns found in nature which are the result of an instability in a dynamical process. (a) columnar joints, (b) ice-wedge polygons, (c) Kinneyia fossil mat, (d) salt polygons, (e) dunes and (f) fairy circles. Images (a), (b) and (c) courtesy Lucas Goehring. Image (f) adapted from [46] and courtesy Stephan Getzin. . . . . . . . . . . . . 10

1.9 Illustration of the advection-diffusion dynamics below the salt polygons. Large red arrows indicate underground liquid movement whereas yellow arrows indicate evaporation through the surface. Downwellings are co-localized with salt ridges at the surface. . . . . . . . . . . . 12

2.1 Schematic representation of a volume $V$ of porous medium with volume $V_{s}$ occupied by the solid matrix and volume $V_{f}$ occupied by the fluid. . . . . . . . . . . . . . . . . . 18

2.2 Images recorded in the first half of 2018 by a camera installed at Owens Lake. (a) the image from March $13^{\text {th }}$ shows the flooded surface corresponding to scenario (1). In this case the underlying crust has not completely dissolved. (b) the image from May $12^{\text {th }}$ shows that the brine has completely evaporated, leaving behind a skeleton crust. (c) the image from July $6^{\text {th }}$ shows the crust with pronounced ridges after significant growth has happened. . . . . . . . . . . . . .

3.1 Illustration of the simulated domain with width $W$, depth $H$ and resolution $\Delta x=\Delta z$. The domain is periodic in the $X$-direction. At the top, the vertical velocity $U_{Z}(Z=0)$ is set. . . . . . . . . . . . . . 29

3.2 Exemplary snapshots of simulations for $R a=20,100$ and 1000 at times $\tau_{i}$. Note the different times $\tau_{i}$ and system sizes. . . . . . . . 36

3.3 (a) Initial condition for the salinity $S$. Configuration of $U_{Z}(X, Z)$ at time $\tau=\Delta \tau$ for (b) a uniform top boundary condition and (c) a modulated top boundary condition. . . . . . . . . . . . . . 37

3.4 Detection of maxima (downwelling plumes) in the salinity profile $S(X, 1,1)$ along a line at $Z=-1$ and at time $\tau=1$ for a simulation run at

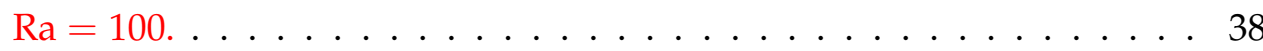

3.5 Sketch of the Hele-Shaw cell setup used in the experiments. Salty water is supplied from the bottom, flows through the cell and evaporates at the top, leaving the salt behind, driving convection in the cell. . . . . . 41

3.6 Experimental setups of sand-filled Hele-Shaw cells. (a) Water invasion front at setup of a cell with $40 \mathrm{~cm}$ width. (b) Installation of two cells with $60 \mathrm{~cm}$ width with water inlets. (c) Salt crust that formed at the top of the right cell. . . . . . . . . . . . . . . . . . 42

3.7 Visualization of flows in the Hele-Shaw cell using three different techniques: (a) coloring from the bottom inlets using fluorescein. (b) coloring from the cell top using rhodamine. (c) coloring from the coloring tube embedded in the middle of the cell using methyl blue. . . . . . . . 43 
3.8 Displaced line of color $175 \mathrm{~min}$ after the start of the coloring experiment. Measured wavelength is indicated in red. . . . . . . . . . . . . 44

3.9 (a) Experimental setup for the 3D experiments with experiment in the middle, water reservoir and fan to the right and camera for timelapse photography to the left. (b)Top-view of the crust after about two weeks of experimental duration. Images courtesy Birte Thiede. . . . .

3.10 Grain size diameters of the artificial sand used in the experiment measured by LPS. . . . . . . . . . . . . . . . . . . . .

3.11 Linear fit to the empirical relationship of porosity to grain size for dry and wet packs of sand determined by [146] . . . . . . . . . . . 50

3.12 (a) Evaporation rates were measured by tracking the water levels in the reservoirs using manometers.(b) Single manometer with black marker strip at the top and colored water in pink. (c) Exemplary fit to a measurement of water level in the reservoir. . . . . . . . . . . . . . . . 52

3.13 Schematic map of Owens Lake in central California, USA. . . . . . . 56

3.14 Schematic map of Badwater Basin in the Death Valley, central California, USA. Sampling sites are indicated as red dots. . . . . . . . . . . 57

3.15 Sampling by (a) digging a trench and then collecting samples from one trench wall and (c) drawing a soil core and then collecting samples from the core. (b) image of the terrestrial laser scanner (TLS) used to record the surface relief and measure the pattern wavelength. . . . .

3.16 Tool to extract pore water from unconsolidated sand saturated with water. . . . . . . . . . . . . . . . . . . . .

3.17 One of three cameras which were installed at Owens Lake in January $2018 \ldots \ldots \ldots \ldots \ldots \ldots \ldots \ldots$

3.18 Exemplary illustration of different types of salt crust and sampling procedure. Sampling locations are indicated with black circles. . . . . .

3.19 Sampling of red Dunaliella Salina from brine and salt crust at several sites at Owens Lake, CA. . . . . . . . . . . . . . . . . . . . . . 67

3.20 Geologic map of Owens Valley, adapted from [3]. . . . . . . . . . . . 71

3.21 Exemplary polygons encountered at Badwater Basin during my field campaign in 2016. (a) and (b) polygons about $500 \mathrm{~m}$ south of the Badwater Basin tourist entrance. (c) small (about $1 \mathrm{~cm}$ high) ridges and (d) old (brown) ridges from before the 2015 flash flood interwoven with younger (white) ridges at the Badwater Basin tourist entrance. . .

4.1 Onset of instability (dots), neutral stability curve (solid line) and most unstable mode (dotted line) for scenario (1), the "salt lake" condition at uniform pressure (red) and scenario (3), the "salt pan" condition at uniform flow rate (blue) . . . . . . . . . . . . . . . . . . 
4.2 Exemplary grain size distributions for samples at different heights $z$ measured by LPS for (a) Badwater Basin and (b) Owens Lake. Images to the right show the original cores from which samples were collected. 80

4.3 Double logarithmic plot of the time $\tau_{S}$ when $S(Z=1) \geq 0.5$ for the first time, over $\mathrm{Ra}-\mathrm{Ra}_{c}$ for $\mathrm{Ra}=20-2000$. Data points (gray) are averages and standard deviations over ensembles with 5-10 runs. Red line: fit $\tau_{S}\left(\mathrm{Ra}-\mathrm{Ra}_{c}\right)=(62.3 \pm 4.1)\left(\mathrm{Ra}-\mathrm{Ra}_{c}\right)^{-1.28 \pm 0.04}$ to ensemble averages weighted by the standard deviations. . . . . . . . . . . 85

4.4 (a) Salinity distribution $S(Z)$ for system ages $\tau_{S}=0.1$ to $\tau_{S}=10$. (b) Length scales $L^{\prime}$ and background salinities $C_{0}$ from fits to the salinity distributions between $Z=0$ and $Z=-0.8$. Simulations were conducted at $\mathrm{Ra}=10^{3}$, measurements are ensemble averages and standard deviations over 8 simulation runs. . . . . . . . . . . . . . 86

4.5 (a) Salinity distribution $S(Z)$ for four different Ra at $\tau=5 \tau_{S}$. (b) Length scale $L^{\prime}$ and background salinity $C_{0}$ for a range of different Ra. Values are ensemble averages and standard deviations over 7-9 simulation runs. . . . . . . . . . . . . . . . . . . 8 87

4.6 Salinity measurements from a Hele-Shaw setup. Values are averages and standard deviations from 9 samples taken at the same depth each.

4.7 Salinity (red dots) measured in the field and fits to determine $L^{\prime}$ and $C_{0}$ for the field sites. Values for $L^{\prime}$ and $C_{0}$ are listed in Tab. 4.5. Field measurements are averages over all samples taken at a specific depth. Error bars are standard deviations. . . . . . . . . . . . . . . . . 91

4.8 Images of four coloring experiments in Hele-Shaw cells visualizing the upwellings and downwellings in the cell. Grain size in the experiments was 100-200 $\mu \mathrm{m}$, Rayleigh numbers at the time of the coloring were (a) $R a=457$, (b) $R a=220$, (c) $R a=1034$ and (d) $R a=563$. Images were taken $125 \mathrm{~min}, 104 \mathrm{~min}, 208 \mathrm{~min}$ and $120 \mathrm{~min}$ after color injection respectively. . . . . . . . . . . . . . . . . . . . . . 94

4.9 Direct measurement of salt concentration in a Hele-Shaw experiment. The cell was dyed with rhodamine first and later colored with fluorescein from below to the visualize flow patterns (background image). Water inflow was then stopped and the cell was dissected layer by layer, taking samples along up- and downwellings. The overlaid colormap shows salinity at each sampling location. . . . . . . . . . . 95

4.10 Two 3D experiments stained with rhodamine and fluorescin and dissected layer by layer to visualize the flows inside the porous medium. Panels (a) and (d) show the layer at $z=18 \mathrm{~cm}$, panels (b) and (e) show the layer at $z=10 \mathrm{~cm}$ and panels (d) and (f) show the layer at $z=2 \mathrm{~cm}$. Upwellings in the middle layer are indicated with green circles, some downwellings have been marked with blue circles for reference. Images courtesy to Birte Thiede. . . . . . . . . . . . . . . 96 
4.11 Salt concentration profile of cross section under a polygon at site T27S. Ridges were situated at $x=30 \mathrm{~cm}$ and $x=210 \mathrm{~cm}$ respectively. The first two rows of samples are not shown, due to $C>50 \mathrm{wt}$.\%, which is unphysical and indicates contamination with salt crystals from the crust. . . . . . . . . . . . . . . . . . . . . .

4.12 Salt concentration profile of cross section under a polygon at site T321-L1 (2). Ridges were situated at $x=30 \mathrm{~cm}$ and $x=195 \mathrm{~cm} . \ldots \ldots$

4.13 Salt concentration profile of cross section under a polygon at site T321-L1 (3). White pixels indicate missing measurements. Ridges were situated at $x=30 \mathrm{~cm}$ and $x=225 \mathrm{~cm} \ldots \ldots \ldots \ldots$

4.14 Salt concentration $C$ normalized by average salt concentration $\langle C\rangle$ for sites T27-S, T32-1-L1 (2) and T32-1-L1 (3). Positions of ridges are indicated with arrows and data points belonging to set 1 are indicated by a blue background. . . . . . . . . . . . . . . . . . . . . . . . . 101

4.15 Wavenumber $a$ and standard deviation $\sigma_{a}$ measured for simulations with $\mathrm{Ra}=20$ - 4000 at short times $\tau=\tau_{\text {s }}$. Measurements are ensemble averages over 5-10 runs for every Ra. . . . . . . . . . . . . . . . . 103

4.16 Wavenumber development over time in a simulation at $\mathrm{Ra}=100$. Wavenumbers were measured at different depths, depths are colorcoded from yellow $(Z=-1)$ to purple $(Z=-90) \ldots \ldots \ldots$. . . . 104

4.17 Development of the salinity distribution at $Z=-1$ for (a) $\mathrm{Ra}=100$ and $(b) \mathrm{Ra}=38$. Coarsening is clearly visible for longer times. . . . . . 105

4.18 Wavenumbers and standard deviations for simulations for a large range of $\mathrm{Ra}=2 \cdot 10^{1}-2 \cdot 10^{3}$ and $\tau=\tau_{S}$ (yellow), $\tau=10 \tau_{S}$ (red) as well as $\tau=50 \tau_{S}$ (gray). Measurements are ensemble averages over 5-10 runs for every Ra. . . . . . . . . . . . . . . . . . . . . 105

4.19 Wavenumber $a$ measured at $Z=-5$ for simulations at $\mathrm{Ra}=200$ and relative system ages $\tau=\tau_{S}$ to $\tau=99 \tau_{S}$. Measured values (red line) and uncertainty (shaded red area) are averages and standard deviations of an ensemble of 9 runs. The blue line is a linear fit to the wavenumbers measured between $\tau=50 \tau_{S}$ and $\tau=99 \tau_{S} . \ldots . . .106$

4.20 (a) Wavenumbers measured in the experiment over Rayleigh numbers color coded by the logarithm of the relative system age $\tau$ expressed in $\tau_{S}$. (b) Wavenumbers over the logarithm of the relative system age $\tau / \tau_{S} .107$

4.21 (a) Wavenumbers over Rayleigh numbers measured in the field at Owens Lake (blue), Badwater Basin (red) and Bristol Playa (yellow). (b), (c) Surface relief measured at two of the trench sites. . . . . . . . . 108

4.22 Ridge height and polygon wavelength measured by TLS in the field. . 110 
4.23 Overview plot of dimensionless wavenumbers measured in the field (blue squares, Owens Lake and Bristol Playa, orange squares Badwater Basin), the experiments (green triangles) and the simulations at early (yellow dots) and late (red dots) times. The lines indicate the theoretically predicted neutral stability curve (black solid line) and the most unstable mode (gray dotted line) . . . . . . . . . . . . . . 110

4.24 Salinity spacetime diagrams for simulations with nonuniform boundary $U_{Z}(X, Z=0)$ at $\mathrm{Ra}=100, A_{\bmod }=0.4$ for different modulation wavenumbers. Positions of the minima and maxima in the modulation are indicated above the diagrams in gray. . . . . . . . . . . . . 113

4.25 Alignment times $T_{A}$ over modulation wavenumbers $a_{\text {mod }}$ for simulations at $\mathrm{Ra}=100$, amplitudes $A_{\text {mod }} \in\{0.01,0.1,0.5,1.0\}$ and wavenumbers $a_{\mathrm{mod}}=0.3-3.76$. Measurements are averages over ensembles of five simulations each, uncertainties are standard deviations. The wavenumber of the most unstable mode $a_{\Omega}(\mathrm{Ra}=100)$ is indicated as dotted gray line. . . . . . . . . . . . . . . . . . . . . . 114

4.26 Alignment times $T_{A}$ over modulation wavenumbers $a_{\bmod }$ for simulations at $\mathrm{Ra}=100$, amplitudes $A_{\text {mod }}=0.01-1.0$ and wavenumbers $a_{\text {mod }}=0.31-3.76 . T_{A}$ from short alignment (light green) to long alignment (dark blue). Data points are $T_{A}$ measurements for single runs for each set of $\left(A_{\text {mod }}, a_{\text {mod }}\right)$. Note that the modulation amplitudes $A_{\bmod }$ on the $y$-axis do not follow a linear scale. . . . . . . . . . . . 114

4.27 (a) Temperature recorded from two sensors placed inside a ridge (dark red) and in the middle of a polygon (light red) at Owens Lake, site

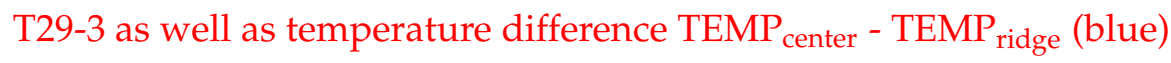
and melting region of Mirabilite (gray area). (b) Relative humidity recorded below a ridge at site T29-3 and in the middle of a polygon at site T36-3 and relative humidity difference $\mathrm{RH}_{\text {center }}-\mathrm{RH}_{\text {ridge }}$ (blue). Measurements were taken every two minutes in a time period of six days between November 26th and December 2nd, 2016 . . . . . . . . 116

4.28 Images of salt polygons at Badwater Basin in winter 2016, about a year after a flood submerged the polygons. The black camera cap used as scale bar is about $5.5 \mathrm{~cm}$ in diameter. (a) Brown ridges are older (potentially from before the flooding of the previous year) and covered in sand. Ridges grow both from the inside of old ridges outwards (b) as well as in between older ridges (c). . . . . . . . . . . . . . 117

4.29 Overnight change from November 25th - 26th 2018. TLS scans were normalized by their mean value. (a) $11 \mathrm{~m} \times 11 \mathrm{~m}$ section of crust at cell T27-A, (b) $11 \mathrm{~m} \times 11 \mathrm{~m}$ section of crust at cell T29-3, (c) $0.4 \mathrm{~m} \times 0.8 \mathrm{~m}$ containing a single ridge from cell T29-3. . . . . . . . . . 118 
4.30 (a) and (b): Site T16 in January and July 2018. (c), (d) and (e): Zoom into bottom left field of view of the camera. (c) crust before flooding, (d) crust completely flooded, (e) ridge emerging from the water. (f), (g) and (h): Evidence for the growth of a polygon ridge by about $5 \mathrm{~cm}$ over the course of 20 days from May $25^{\text {th }}$ to July $4^{\text {th }} 2018 \ldots \ldots \ldots$

4.31 Images of a $2 \times 4$ section of salt crust at Owens Lake at (a) January $17^{\text {th }}$ and (b) July $7^{\text {th }}$. (c) Indication of ridge location before (red) and after (blue) flooding. . . . . . . . . . . . . . . . . . 120

5.1 Illustration of the different regimes of the dynamics. The panels show the salinity field $S \in[0,1]$ with $S=0$ in yellow and $S=1$ in purple for a simulation at $\mathrm{Ra}=100$ with a system size of $40 \times 40 \mathrm{~L}$. (a) Onset of instability at $\tau=0.1 \tau_{S}$, (b) flux growth regime at $\tau=2 \tau_{S}$, (c) merging regime at $\tau=6.5 \tau_{S}$, (d) re-initiation regime at $\tau=15.5 \tau_{S}-$ note the small nascent plumes at the top boundary and (e) increasing saturation of the system at $\tau=125 \tau_{s} \ldots \ldots \ldots \ldots \ldots$. . . . . . 128

5.2 Content of samples taken at site T27-S. Panel (a) shows the salinity. Panel (b) shows pore water content in wt.\%. Panel (c) shows sand content per sample in grams. . . . . . . . . . . . . . . . . 135 135

5.3 Samples from site T36-3 (3) collected during the first field campaign in 2016 were split into two equal parts before being processed in the laboratory. Panels (a) and (b) show the salinity values measured for each of the two sample groups and allow for a comparison of the accuracy of salinity measurements. Color code indicates salinity in wt.\% of pore water. . . . . . . . . . . . . . . . . . 137

5.4 Organic matter proportions for the salt (red) and sand (orange) part of samples from site T32-1-L1 (1) over depth. . . . . . . . . . . . . 138

6.1 Snapshots of planforms at $Z=-1$ in three-dimensional simulations of the buoyancy driven porous media convection at $\mathrm{Ra}=100$, conducted by Cédric Beaume. The colormap indicates salinity $S \in[0,1]$. (a) Early stage of the simulation shortly after the onset of convection. (b) Simulation after some coarsening showing convection cells very similar to the ridge patterns observed in nature. (c) and (d) late stages of the simulation, showing a transition to square convection cells. Images courtesy to Cédric Beaume, University of Leeds. . . . . . . . . . . 146

6.2 Ideas for improved and novel experimental setups. (a) A circular Hele-Shaw cell and (b) modulation of the evaporation rate with polystyrene blocks. . . . . . . . . . . . . . . . . . . . . . . 147 
C.1 Eucaryotic Algae and Cyanobacteria: (a) Filamentous Cyanobacteria of genus Leptolyngbya (Leptolyngbya sp.) and green algae of genus Dunaliella (Dunaliella sp.) (b) Diatoms of genus Nitzschia (Nitzschia sp.); Green Algae Dunaliella sp. and Nannochloris sp. (c) Dunaliella sp. and Nitzschia sp. (Bacillariophyta) (d) Nannochloris sp., Dunaliella sp. and Nitzschia sp. (e) Dunaliella sp. (f) Nannochloris sp. and Dangeardinella sp. Images courtesy Maike Lorenz, Göttingen Algae Collection. . . . . . . . . . . . . . . . . . . . . . 183

C.2 Eucaryotic Algae, Cyanobacteria and Protistan predators: (a) Halothece californica (Cyanobacteria) (b) Synechococcus sp (Cyanobacteria) (c) Halothece Californica (d) Cysts of cyanobacterium Nostoc sp. (e) Ciliophora (formerly called Ciliata (Wimpertierchen)) feeding on algae (f) Amoeba feeding on algae. Images courtesy Maike Lorenz, Göttingen Algae Collection. . . . . . . . . . . . . . . . . . . . . . . . . . . 184 


\section{List of Tables}

3.1 Boundary conditions for the salinity $S$ stream function $\psi$ and vertical velocity $U_{Z}$ in case of a uniform (left) and non-uniform (right) flow rate at the top. . . . . . . . . . . . . . . . . . 31

3.2 System dimensions $H \times W$ and spatial and time resolutions $\Delta X$ and $\Delta T$ respectively of simulations for different Ra . . . . . . . . . . . 35

3.3 Nomenclature of soil with different grain diameters from the UddenWentworth scale [136]. . . . . . . . . . . . . . . . . . . . 46

3.4 Permeabilities measured for the sand beads used in the Hele-Shaw cell experiments measured by $[135] . \ldots \ldots \ldots \ldots$. . . . . . . .

4.1 Critical Rayleigh number and wavenumber for the "salt lake" and "salt pan" boundary conditions corresponding to scenarios constant pressure and constant flux described in Sec. 2.6. . . . . . . . . . . . . . 78

4.2 Background and maximum densities measured from pore water extracted at the field sites at Owens Lake and measured using a vibratingtube densimeter. . . . . . . . . . . . . . . . . . . .

4.3 Sauter diameter $d_{S}$, permeability $\kappa$, porosity $\phi$ and Rayleigh number Ra measured or calculated for each of the field sites at Owens Lake, Badwater Basin and Bristol Playa. Samples stemming from a trench are indicated as $(*) \ldots \ldots \ldots \ldots \ldots \ldots$

4.4 Sauter diameter $d_{S}$, permeability $\kappa$, porosity $\phi$ Rayleigh number Ra for each of the experimental setups.

4.5 Length scale and background salinity determined from fits to the measured salinity gradient for nine field sites. Ra is given for reference (see Tab 4.3 for error ranges) and $N_{X}$ denotes the number of samples for each depth $Z . \ldots \ldots \ldots \ldots \ldots \ldots \ldots$

4.6 Values for $E, L, \tau$ and Ra and measurement of the wavenumber $a$ for the four exemplary experiments shown in Fig. 4.8.

4.7 Pattern wavelength $\lambda$, dimensionless wavenumber $a$ and ridge height $h$ measured by TLS for most of the field sites at Owens Lake (filled squares) and Badwater Basin and Bristol Playa (open squares). . . . . . 109

5.1 Evaporation rate and Péclet number calculated following Eq. (5.1) for the minimum, average and maximum velocity in the experiment as well as Owens Lake and Badwater basin. . . . . . . . . . . . . . . 126 
5.2 Redundant samples collected at site T16 by coring. (V) indicates measurements from samples taken for validation purposes. . . . . . . . . 136

A.1 Sites visited in 2016 were consistently sampled with a lower resolution but more sites were covered. The second campaign focused on fewer experiments but with a much higher resolution. . . . . . . . . . 151

A.2 Particle size and water content for site Badwater Basin (1). Layer is specified as distance from surface. . . . . . . . . . . . . . . . 152

A.3 Particle size and water content for site Badwater Basin (1). Layer is specified as distance from surface. . . . . . . . . . . . . . 153

A.4 Particle size and water content for site Badwater Basin (1). Layer is specified as distance from surface. . . . . . . . . . . . . . . . 154

A.5 Particle size and water content for site Badwater Basin (1). Layer is specified as distance from surface. . . . . . . . . . . . . . . 155

A.6 Particle size and water content for site Badwater Basin (1). Layer is specified as distance from surface. . . . . . . . . . . . . . . 156

A.7 Particle size and water content for site Bristol Playa. Layer is specified as distance from surface. . . . . . . . . . . . . . . . . 157

A.8 Particle size and water content for site T10-3. Layer is specified as distance from surface. . . . . . . . . . . . . . . . . 158

A.9 Particle size and water content for site T16. Layer is specified as distance from surface. . . . . . . . . . . . . . . . . . . . 159

A.10 Particle size and water content for site T2-4. Layer is specified as distance from surface. . . . . . . . . . . . . . . . . . 160

A.11 Particle size and water content for site T2-5 (1). Layer is specified as distance from surface. . . . . . . . . . . . . . . . . . 161

A.12 Particle size and water content for site T2-5 (2). Layer is specified as distance from surface. . . . . . . . . . . . . . . . . . . 162

A.13 Particle size and water content for site T2-5 (3). Layer is specified as distance from surface. . . . . . . . . . . . . . . . . . . 163

A.14 Particle size and water content for site T25-3 (1). Layer is specified as distance from surface. . . . . . . . . . . . . . . . . . . . . . 164

A.15 Particle size and water content for site T25-3 (2). Layer is specified as distance from surface. . . . . . . . . . . . . . . . . . . 165

A.16 Particle size and water content for site T27-A (1). Layer is specified as distance from surface. . . . . . . . . . . . . . . . 166

A.17 Particle size and water content for site T27-A (2). Layer is specified as distance from surface. . . . . . . . . . . . . . . . . . 167

A.18 Particle size and water content for site T27-A (3). Layer is specified as distance from surface. . . . . . . . . . . . . . . . . . . 168

A.19 Particle size and water content for site T27-S. Layer is specified as distance from surface. . . . . . . . . . . . . . . . . . . . . 169 
A.20 Particle size and water content for site T29-3 (1). Layer is specified as distance from surface. . . . . . . . . . . . . . . . . . . . . 170

A.21 Particle size and water content for site T29-3 (2). Layer is specified as distance from surface. . . . . . . . . . . . . . . . . . . 171

A.22 Particle size and water content for site T32-1-L1 (1). Layer is specified as distance from surface. . . . . . . . . . . . . . . . . . . . 172

A.23 Particle size and water content for site T32-1-L1 (2). Layer is specified as distance from surface. . . . . . . . . . . . . . . . . . . . . 173

A.24 Particle size and water content for site T32-1-L1 (3). Layer is specified as distance from surface. . . . . . . . . . . . . . . . . . . . . . 174

A.25 Particle size and water content for site T36-3 (1). Layer is specified as distance from surface. . . . . . . . . . . . . . . . . . . 175

A.26 Particle size and water content for site T36-3 (2). Layer is specified as distance from surface. . . . . . . . . . . . . . . . . . 176

A.27 Particle size and water content for site T36-3 (3). Layer is specified as distance from surface. . . . . . . . . . . . . . . . . 177

A.28 Particle size and water content for site T8-W. Layer is specified as distance from surface. . . . . . . . . . . . . . . . . . . 178

B.1 List of minerals found in saline pore water and salt crust, their chemical composition, solubility at $20^{\circ} \mathrm{C}$ and diffusion rate for single ion diffusion at $20^{\circ} \mathrm{C}$ and infinite dilution. . . . . . . . . . . . . . 179

B.2 Analysis of minerals in the pore water samples using quantitative $\mathrm{XRD}$, proportions are given in mass \%. One to three samples per area / management cell were analyzed. Only the four most common minerals are listed. Minerals with a proportion $<1 \%$ are not listed. Amorphous proportions were interpreted as Mirabilite. . . . . . . . . . 180

B.3 Analysis of minerals from samples taken from the crust using qualitative XRD, proportions are given in \% mass. Only samples from site T2-5 and T10-3 were analyzed to compare results with the pore water minerals listed in table B.2. Only the four most common minerals for each sample are listed. Minerals with a proportion $<1 \%$ are not listed. 181

B.4 Total dissolved salts analyzed by a) quantitative XRD (subscript "X") and b) ICP-OES (subscript "I"). Values in red exceeded the calibration value of the ICP-OES and could potentially be significantly underestimated. Concentrations of Arsenic as measured with the ICP-OES are added to the table, since they are of interest as potential health hazard. 



\title{
List of Abbreviations
}

\author{
CPU Central Processing Unit \\ ERT Electrical Resistance Tomography \\ GPU Graphics Processing Unit \\ ICP-OES Induced Coupled Plasma - Optical Emission Spectroscopy \\ LPS Laser Particle Sizer \\ TLS Terrestrial Laser Scanner \\ XRD X Ray Diffraction
}





\section{Physical Constants}

Density of water at $25^{\circ} \mathrm{C}$

Diffusion coefficient of $\mathrm{NaCl}$

Diffusion coefficient of $\mathrm{Na}_{2} \mathrm{SO}_{4}$

Gravity

$\mathrm{Pi}$

$$
\begin{aligned}
\rho_{0} & =997 \mathrm{~kg} \mathrm{~m}^{-3} \\
D_{\mathrm{NaCl}} & =1.61 \cdot 10^{-9} \mathrm{~m}^{2} \mathrm{~s}^{-1} \\
D_{\mathrm{Na}_{2} \mathrm{SO}_{4}} & =0.57 \cdot 10^{-9} \mathrm{~m}^{2} \mathrm{~s}^{-1} \\
g & =9.81 \mathrm{~m} \mathrm{~s}^{-2} \\
\pi & =3.14
\end{aligned}
$$





\section{List of Symbols}

\begin{tabular}{|c|c|c|}
\hline$A_{\text {mod }}$ & modulation amplitude & \\
\hline$a$ & dimensionless wavenumber & \\
\hline$a_{c}$ & critical dimensionless wavenumber & \\
\hline$b$ & spacing of plates in Hele-Shaw experiments & $\mathrm{m}$ \\
\hline C & salt concentration & wt. \% \\
\hline$c_{m}$ & salt mass fraction & \\
\hline$c_{v}$ & brine volume fraction & \\
\hline$D$ & molecular diffusion Coefficient & $m^{2} s-1$ \\
\hline$d$ & grain diameter & $\mathrm{m}$ \\
\hline$d_{S}$ & Sauter diameter & $\mathrm{m}$ \\
\hline$E$ & evaporation rate & $\mathrm{ms}^{-1}$ \\
\hline$H$ & dimensionless system depth & \\
\hline$h$ & ridge height & $\mathrm{m}$ \\
\hline$L$ & natural length scale & $\mathrm{m}$ \\
\hline$L^{\prime}$ & length scale of the salinity gradient & \\
\hline$M$ & number of grid points in the $X$-direction & \\
\hline$N$ & number of grid points in the Z-direction & \\
\hline $\mathrm{Nu}$ & Nusselt number & \\
\hline$P$ & dimensionless pressure & \\
\hline$p$ & pressure & $\mathrm{kg} \mathrm{m}^{-1} \mathrm{~s}^{-2}$ \\
\hline$q$ & fluid flux & $\mathrm{ms}^{-1}$ \\
\hline $\mathrm{Ra}$ & Rayleigh number & \\
\hline $\mathrm{Ra}_{c}$ & critical Rayleigh number & \\
\hline $\operatorname{Re}$ & Reynolds number & \\
\hline$S$ & salinity & \\
\hline$T$ & natural time scale & $\mathrm{s}$ \\
\hline$T_{A}$ & alignment time & \\
\hline$\Delta T$ & time resolution & \\
\hline$t$ & time & $\mathrm{s}$ \\
\hline$U$ & dimensionless fluid flux & \\
\hline$U_{X}$ & dimensionless velocity in the $X$-direction & \\
\hline$U_{Z}$ & dimensionless velocity in the $Z$-direction & \\
\hline$V$ & volume & $\mathrm{m}^{3}$ \\
\hline$V_{f}$ & fluid occupied volume & $\mathrm{m}^{3}$ \\
\hline$V_{s}$ & solid occupied volume & $\mathrm{m}^{3}$ \\
\hline$v$ & fluid velocity & $\mathrm{ms}^{-1}$ \\
\hline W & dimensionless system width & \\
\hline$X, Y, Z$ & dimensionless Cartesian coordinates & \\
\hline$\widehat{X}, \widehat{Y}, \widehat{Z}$ & dimensionless unit vectors & \\
\hline$\Delta X$ & resolution in the $X$-direction & \\
\hline$\Delta Z$ & resolution in the $Z$-direction & \\
\hline$x, y, z$ & Cartesian coordinates & $\mathrm{m}$ \\
\hline
\end{tabular}


xxxii

$\begin{array}{lll}\hat{x}, \hat{y}, \hat{z} & \text { unit vectors } & \\ \theta & \text { tortuosity } & \\ \kappa & \text { permeability } & \mathrm{m}^{2} \\ \kappa^{\prime} & \text { dimensionless permeability } & \\ \Lambda & \text { dimensionless wavelength } & \mathrm{m} \\ \lambda & \text { wavelength } & \\ \lambda_{\text {mod }} & \text { modulation wavelength } & \mathrm{kg} \mathrm{m}^{-1} \mathrm{~s}^{-1} \\ \mu & \text { dynamic viscosity } & \mathrm{kg} \mathrm{m}^{-3} \\ \rho & \text { density } & \mathrm{kg} \mathrm{m}^{-3} \\ \rho_{S} & \text { density of brine } & \mathrm{kg} \mathrm{m}^{-3} \\ \rho_{b} & \text { background density } & \mathrm{kg} \mathrm{m}^{-3} \\ \Delta \rho & \text { density difference } & \\ \sigma & \text { standard deviation } & \\ \tau & \text { dimensionless time } & \\ \tau_{S} & \text { relative system age } & \\ \Delta \tau & \text { time integration step size } & \\ \phi & \text { porosity } & \\ \psi & \text { stream function } & \\ \widetilde{\psi} & \\ \psi^{0} & \text { Fourier coefficients } & \\ \widetilde{\psi}, \widetilde{\omega} & \text { constant component of } \psi & \text { rotational component of } \psi \\ \omega & \text { vorticity } & \\ \omega^{0} & \text { constant component of } \omega & \\ \widetilde{\psi}, \widetilde{\omega} & \text { rotational component of } \omega & \end{array}$


xxxiii 
xxxiv

This work is dedicated to my family, who always held me but never held me back.

Ich widme diese Arbeit meiner

Familie, die mir immer Halt gegeben aber mich nie zurückgehalten hat. 


\section{Chapter 1}

\section{Introduction}

Salt deserts are among the most inhospitable places on the surface of the earth. They emerge in arid environments, where the sun's radiation relentlessly burns away the scarce water, and minerals which accumulated there over millennia, effloresce in beautiful forms. Described in geological terms, salt deserts, salt pans or salt playa - which all describe the same landform - are geological

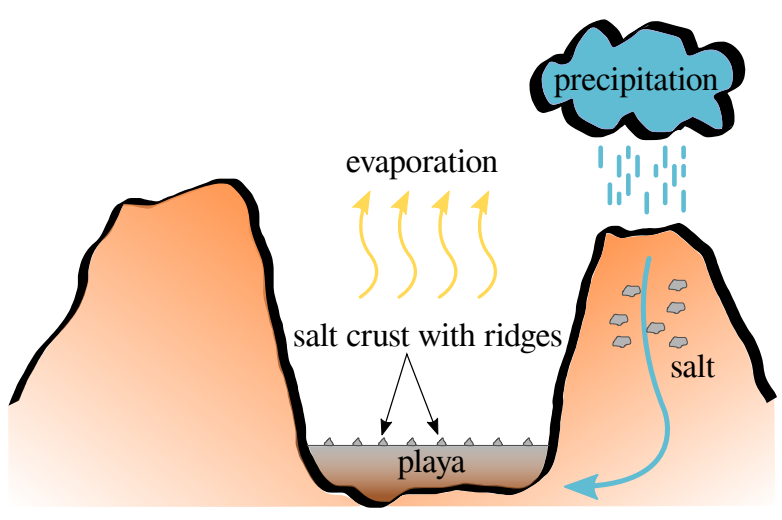

FIGURE 1.1: Schematic representation of salt playa as a geographical sink.

sinks, where evaporation by far outweighs precipitation [1, 2]. The general geological setting is illustrated in Fig. 1.1. Salt pans often are surrounded by high mountains, which prevent clouds from carrying the atmospheric water into the region [3]. Nevertheless, the water precipitated in the mountains percolates through the rock and finds its way into the sink, carrying with it the minerals it dissolved on the way. As the water evaporates again, it leaves behind the minerals, which form a stable crust $[4,5]$.

At the salt playas I visited for this study, the crust, which has a thickness of about $2-20 \mathrm{~cm}$, rests on a bed of unconsolidated sand, which is a product of fluvial deposition. The crust and the patterns of crystallized salt which emerge on it are the interest of this work. Patterns include flat surfaces with centimeter-scale structures like fingers, squares and small ridges, as illustrated in Fig. 1.2 (a) to (c), whose characteristics depend on the precipitated minerals. On the scale of tens of centimeters, the crust shows domes, bumps and winding forms, as illustrated in Fig. 1.2 (d) to (f), greatly increasing the roughness of the surface and its interaction with the wind blowing over the it. On the scale of meters, the crust often shows ridges, rising above the flat crust by up to $30 \mathrm{~cm}$, further increasing the roughness of the surface. These ridges form the iconic regular hexagonal patterns with a characteristic spacing of about one meter. 

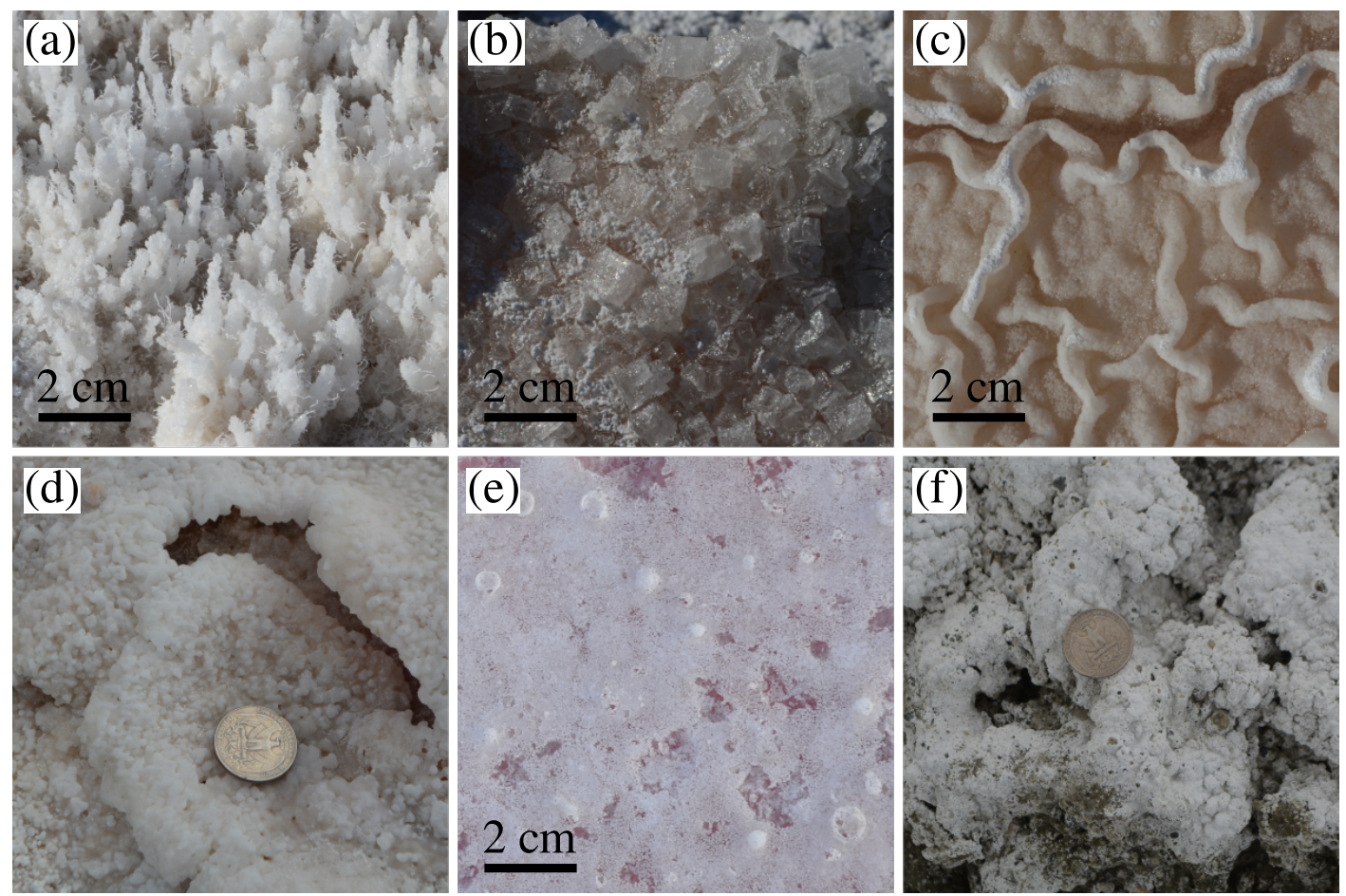

FIGURE 1.2: Exemplary salt crust patterns found in salt pans at the west coast of the US. Panels (a), (b) and (c) show fingers, squares and small ridges respectively. Panels (d), (e) and (f) show a dome, small bumps and winding precipitation patterns respectively. Dollar coins were placed in the images for scale.

The polygons immediately draw the eye of an observer and the question of their origin arises - yet the nature of the driving mechanism responsible for their emergence remains unknown. The driving mechanism has to be rather robust to changing circumstances, since it expresses the same length scale and shape in a variety of surroundings on several continents. Here I propose that the patterns visible on the surface are the product of a dynamical process: convection, which takes place in the ground below the patterns.

In the following introduction to my work, I will illustrate the connection between salt playa and atmospheric dust, as well as the influence of humans on this landform and vice versa. Moreover I will motivate how the methodologies and concepts from other fields of research, such as $\mathrm{CO}_{2}$ sequestration, are used in this work. While applications to climate modelling can most certainly be found, I perceive this work as contribution to the original and, in my opinion most important, driving mechanism of science itself: the satisfaction of human curiosity.

Later in this chapter I will introduce a physical model of salt playa as a dynamical process, described by the motion of water and salt in a porous medium. This puts my work in a line of successful attempts which explain and describe geomorphological pattern formation processes by means of instability in a dynamical system. The model, as well as numerical simulations of it, will then allow me to make predictions about the system, which are verifiable both in experiments as well as in the field, where the phenomenon of salt polygons occurs naturally. 
Last I will review the existing literature on similar approaches to model and verify dynamical processes in a geological or geomorphological setting. Here the connection to $\mathrm{CO}_{2}$ sequestration and the modeling of waste seepage into the underground will become apparent.

\subsection{Salt deserts: a common landform}

Salt polygons emerge in many regions of the world, independent of local conditions. Examples of regions where polygons can be found include

- the East coast of the USA, specifically Bristol Playa, Mono Lake, the Great Salt Lake, Owens Lake and Badwater Basin, which is shown in Fig. 1.3 (a),

- the Salar de Uyuni, Chile, shown in Fig. 1.3 (b),

- Namak Lake, Iran, shown in Fig. 1.3 (c),

- the Dead Sea, Israel [6], shown in Fig. 1.3 (d),

- the Chott el Djerid, Tunisia [7] and

- the Makgadikgadi Pans, Botswana [8].
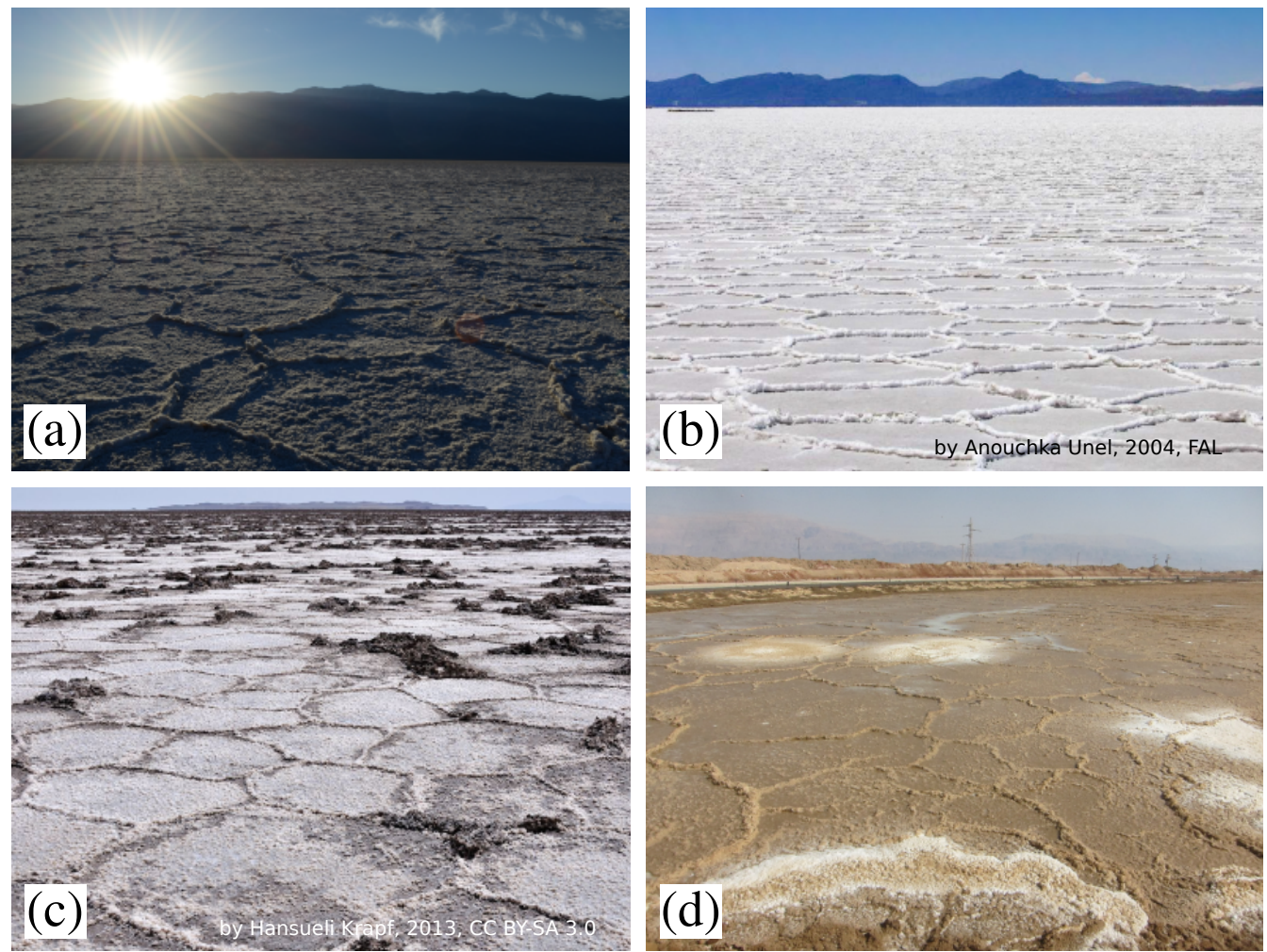

FIGURE 1.3: Salt polygons found in several salt deserts all over the world: (a) Badwater Basin in the Death Valley, California, USA, (b) Salar de Uyuni, Chile, (c) Namak Lake, Iran and (d) the Dead Sea, Israel, image courtesy Lucas Goehring. Polygons visible in the photographs have a diameter of 1-2 meters. 
It is important to note, that in between these regions, local conditions vary significantly. While salt pans in the Andes in Chile exist at high altitudes and in rather cold climates, salt pans at the west coast of the USA are located at or below sea level and experience extremely hot temperatures. Some salt pans, such as the ones found at the coast of the Dead Sea, exist in rather wet conditions, whereas others such as the Chott el Djerid and Namak lake exist in very dry conditions and are subject to rare but violent precipitation events.

Even though conditions vary widely, the emerging patterns and their length scales are very similar. All these salt pans show regular polygonal ridges with a wavelength of about 1-2 m on their surface. Even after inundation events, where the complete crust is dissolved and all patterns are erased, the polygonal patterns quickly form again within the course of a few months to a year [8]. This indicates that the patterns are a product of a quite robust process which drives their emergence.

\subsection{Importance of salt pans for climate and human livelihood}

\subsubsection{Climate-surface interactions}

Several characteristics, such as the existence of cracks in the crust, the position of the water table and the increase of surface available to evaporative processes, influence moisture and heat fluxes on the playa surface [9]. This in turn influences the surface energy balance of the desert region [10]. Since land is a key contributor to energy balance based climate modeling [11, 12], a better understanding of the processes influencing the geomorphology of salt deserts will contribute to the better understanding of the earth's climate.

According to $[13,14,15]$, salt deserts are a common landform, responsible for a significant part of the global emission of atmospheric dust. The height and composition of polygonal ridge patterns on salt playa is a main contributor to surface roughness [8]. Under the effect of the often strong winds which blow over the playa surface, dust is emitted and carried into the atmosphere. The surface roughness, alongside the salt chemistry and other crust characteristics, greatly influences the uncertainty in modeling dust emission from the playa surface [16, 17]. Therefore, understanding and predicting surface roughness depending on the characteristics of the region will help to understand the dust emission potential of a salt pan.

The impact of dust in the atmosphere is plentiful and well-studied. The most prominent effect is probably the influence of dust on the formation of rain clouds [18]. Small particles are carried away by the wind, lifted up into higher layers of the atmosphere and act as nucleation cores for rain droplets, which in turn are the basis of larger weather phenomena. Moreover, dust has a well documented detrimental effect on human health [19]. 


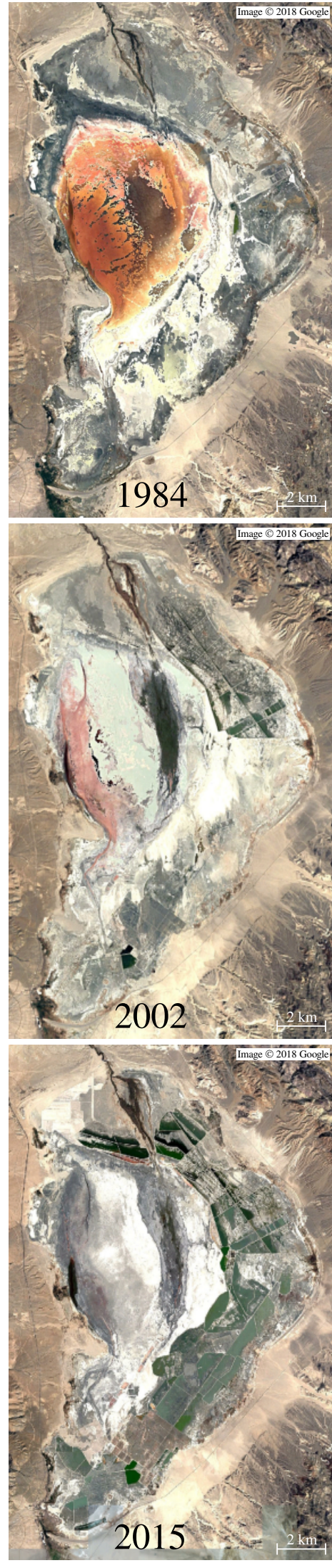

FIGURE 1.4: Implementation of the dust management project (source: Google Earth).
A second important contribution of dust to the understanding of climate is its impact on the reflection of sunlight in the earth's atmosphere. Dust is an aerosol and affects the albedo of the earth's atmosphere by its presence. Aerosols can both decrease [20] and increase [21] global mean temperatures - depending on their properties. Therefore, an understanding of the composition and quantity of dust emitted from the surface of salt pans is crucial for the modeling of aerosol impact on atmosphere albedo.

Finally, atmospheric dust is a important contributor of mineral transport to the sea $[22,23]$. In the sea it has far-reaching effects, for example on the growth and bloom of algae.

\subsubsection{Human-surface interactions}

I conducted two field campaigns to investigate polygonal patterns in nature in 2016 and 2018. During the campaigns I visited the Death Valley, Bristol Playa Soda Dry Lake and Owens Lake, four salt pans located at the west coast of the USA. Especially the salt crusts at Owens Lake have an increased importance for the interaction between nature and humans - since they are a product of human interference and significantly interfere with human lives.

In the following, I will describe the history and current state of Owens Lake since it illustrates how a better understanding of nature could have helped to prevent a natural disaster, and how it is helping now to remedy its detrimental impact on humans living in the region. Moreover I spent the largest part of both two two-week long field campaigns at Owens Lake, and most of the field data presented in this work originates from there.

\section{Owens Lake history}

Owens Lake is located about 300 kilometers North of Los Angeles in the pleasant Owens Valley at the West coast of the USA. Owens Valley is the deepest valley in the United States, bounded in the West by the Sierra Nevada and in the north-east by the White Mountains. 
The valley itself receives very little precipitation: In the years 1934-1985, the town of Lone Pine, directly North of Owens lake received an average of about $100 \mathrm{~mm}$ of annual precipitation [3]. Clouds rarely surpass the high mountains on either side of the valley and release the water they carry to the mountains in the form of rain or snow. The runoff from the mountains feeds the Owens River which terminates in Owens Lake.

Until around 1900, the lake used to be a water basin where incoming water and evaporation from the lake's surface were balanced. As the runoff from the mountains carried a substantial amount of minerals with it, the lake water accumulated an increased salt concentration naturally [24].

At the turn of the century, the Los Angeles aqueduct was built and started to divert most of the water of the Owens River to sustain the ever-growing population of the city of Los Angeles. As the equilibrium between evaporation and inflowing water was disturbed, the lake started to shrink. Around 1925 the lake had completely vanished, giving way to a large arid wasteland where the salt, which used to be dissolved in the lake's water, effloresced and formed a fluffy crust.

\section{Toxic dust}

Owens Valley is subject to strong winds along the North-South axis of the valley [25]. The wind carries small sand particles from the former lake's shores over the salt flats and degrades the salt crust further, carrying the scoured minerals with it. As a result, Owens Lake became North America's single largest source of atmospheric dust [9]. The minerals accumulated on the lakes surface also contain significant amounts of sulfates [26] and arsenic [27, 28]. Sulfates and other salts are abraded from the surface by the saltating particles and carried downwind [29, 30, 31, 32]. Air pollution by the sulfates and arsenic is problematic for the environment and detrimental to the health of the human population living downwind of the lake [13].

\section{Surface management}

As the city of Los Angeles was initially responsible for diverting the water which sustained the lake, it also carried the responsibility of managing the dust originating from the lakebed. The city was ruled to implement measures to alleviate the dust problem, caused by the now dry Owens Lake. This led to a decades-long, large-scale effort to manage the salt flats covering the surface of the now dry lake [33]. Since the year 2000, roughly one billion dollars ${ }^{1}$ was spent to reduce dust emission from Owens Lake. The implementation of the management project on the east side of the lake is clearly visible in the satellite images from 1984 to 2015, displayed in Fig. 1.4. The lakebed is subdivided into cells, mostly situated at the east side of the lake. In each cell, one of a selection of different dust control measures is implemented and its effectiveness is monitored. The different management measures include

\footnotetext{
${ }^{1}$ information by local staff of the Los Angeles Department of Land and Power
} 
- shallow flooding [34], shown in Fig. 1.5 (a),

- growth of salt-resistant vegetation [35], shown in Fig. 1.5 (b) and (f) left side,

- coverage with gravel [33], shown in Fig. 1.5 (c),

- digging of deep trenches, shown in Fig. 1.5 (e) and

- coverage with artificial salt crusts, shown in Fig. 1.5 (d), (f) right side and (g).
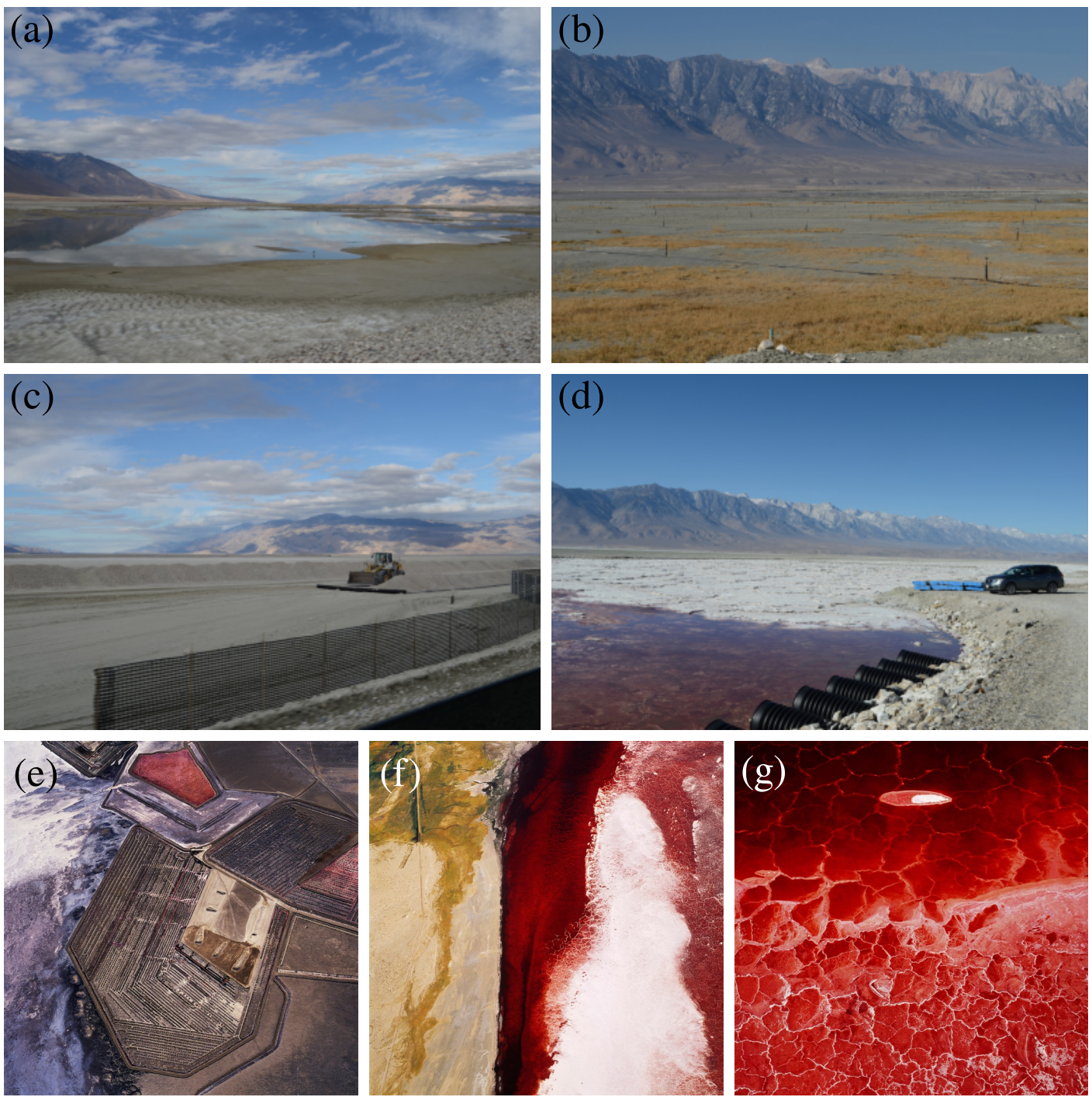

FIGURE 1.5: The surface of Owens Lake is heavily managed to prevent dust emission by (a) shallow flooding, (b) plantation of salt resistant vegetation, (c) coverage with gravel, (d) flooding with brine and subsequent formation of an artificial and more stable salt crust and (e) trenching. (f) shows the sharp transition between a cell managed with vegetation and a brine pool and $(\mathrm{g})$ shows the polygonal structures emerging from a brine pool. The red color is due to a bloom of the algae Dunaliella Salina, which finds a natural habitat in the salt brine. Images (e), (f) and (g) courtesy David Meisel. 
Fresh water is a scarce resource in California. Therefore, the different lake management measures aim to reduce fresh water usage, while optimizing the cost and longevity of measures. Because of their water use, shallow flooding and vegetation have been deemed the least efficient measures, as they require constant irrigation $[35,34,36]$. Sections which have been subjected to trenching are expected to require regular attention, as the trenches will be degraded by the wind over time ${ }^{2}$. Coverage with gravel is very expensive and also requires regular cleaning of the gravel $[33,36]$, as the surface tends to clog with sand, which is carried inwards from the lake shores by the wind. The last available measure is flooding with brine, which subsequently forms an artificial salt crust. In contrast to naturally forming crust, the artificial crust is composed of a different mixture of minerals, which effloresce differently. This leads to a more solid and less fluffy crust, which is less prone to emit dust particles in high winds. So far, coverage with an artificial salt crust has been shown to yield sufficient results in terms of dust reduction, does not use large amounts of fresh water and is expected to require less maintenance than the other measures [37].
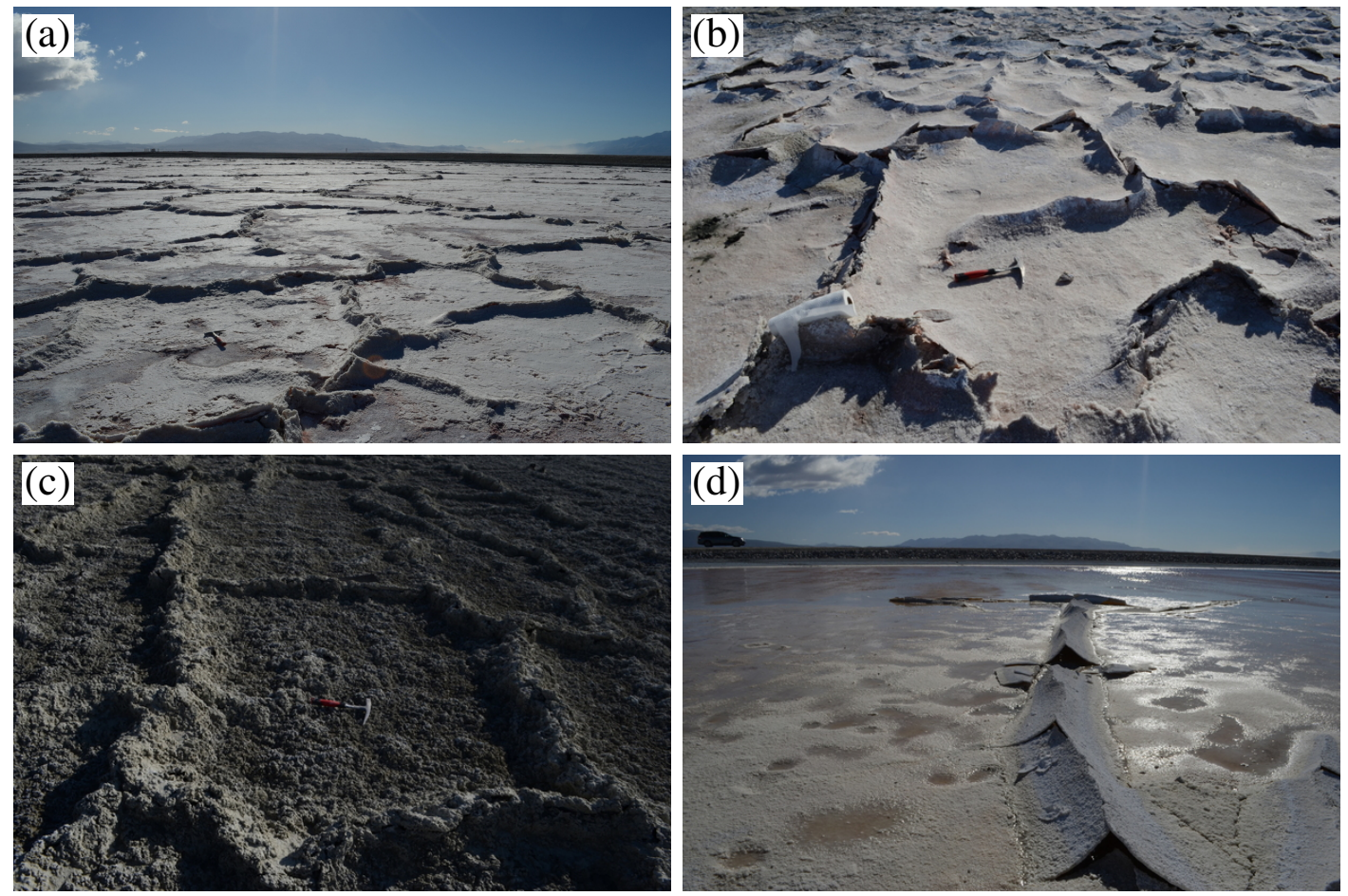

FIGURE 1.6: Exemplary polygons from Owens Lake. (a) and (b) polygons and continuous crust at an older managed site. (c) polygons at an unmanaged site near the east shore of the lake. (d) tepee-like ridge at a recently flooded site. The handle of the rock hammer shown for scale has a length of $31 \mathrm{~cm}$.

The artificial crusts show the same polygonal patterns on their surface as described in Sec. 1.1. The patterns have similar length scales and ridge heights as their naturally grown counterparts. In the east and west, where the lakebed borders the surrounding mountains, unmanaged areas which were not subjected to dust control measures still exist. These areas also show polygonal patterns, although not in the

\footnotetext{
${ }^{2}$ information by local staff of the Los Angeles Department of Land and Power
} 
iconic and pristine white colors, since they are covered with sand, deposited there by wind. Figure 1.5 (g) shows the view of the flooded brine pools and resulting polygonal structures from a plane. Ground views of some polygons I encountered at Owens Lake are shown in Fig. 1.6. Panels (a), (b) and (d) show artificially grown crusts where panel (c) shows polygons at an unmanaged part of the lakebed. In my two field campaigns, I investigated both polygons in managed and unmanaged areas of the lake.

Different sections of the lakebed are easily accessible via gravel roads and the wind speed, temperature and relative humidity of the area is closely monitored as part of the dust control project. This makes Owens Lake a very suitable location for an in-depth study of salt crusts and their patterns, such as this work.

\subsubsection{Aesthetics and human curiosity}

While a connection of salt polygons to climate-surface modeling is certainly warranted, my main motivation to study these fascinating patterns is the satisfaction of pure human curiosity. Symmetry and order are key ingredients of our perception of aesthetics [38]. When nature creates symmetry, humans feel naturally drawn to these phenomena and the patterns incite curiosity and the wish to explain their origin.

Salt polygons, especially those at Badwater Basin and in the Salar de Uyuni, attract a substantial number of tourists every year. Most recently, the Salar de Uyuni was featured as the inspiration for the planet "Crait" in episode VIII of the movie Star Wars: the last Jedi. The movie uses the iconic landscape created by the polygons of the Salar to create the otherworldly impression of a different planet, as shown in Fig. 1.7.

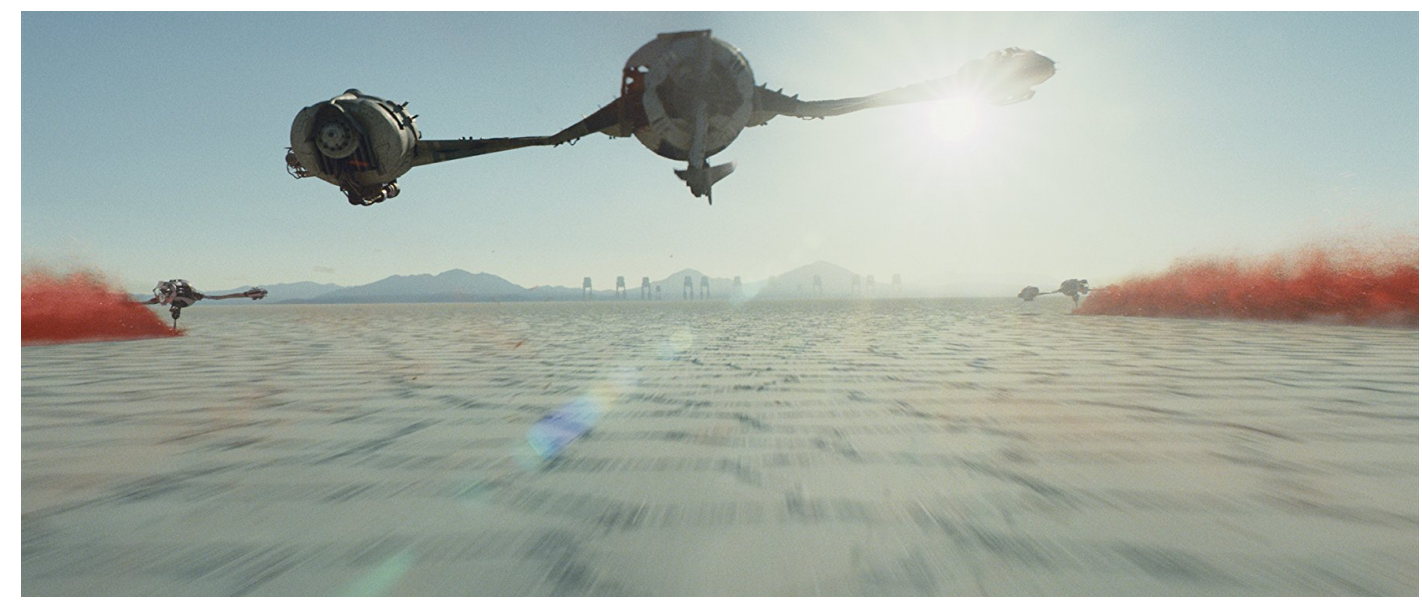

FIGURE 1.7: Image from the Movie Star Wars: the Last Jedi [39], featuring the planet "Crait" with its otherworldly looking landscape. The movie was shot in 2017, the depicted scene is inspired the Salar de Uyuni, Chile. The iconic salt polygons of the Salar are visible in the bottom half of the image.

The strong interest of tourists and artists in these landscapes warrants a closer look by the scientifically minded observer: The drive to explain the unexplained 
and to understand the unexpected and unintuitive order in nature, has for a long time been a major driver of scientific progress. Especially the fact that these patterns can be visited, and their particularity recognized by any layperson or child, makes them a strong ambassador for the pursuit of the scientific method, and the humble goal to preserve what we can from the beauty of nature that still remains.

As a side note: Even the clouds of red dust whipped up by the fighter planes flying low over the salt pan (shown to the left and right in Fig. 1.7) have a natural origin. Salt pans are the habitat of the algae Dunaliella Salina, which lives under the white salt crust and produces large amounts of $\beta$-Carotene [40], and with it a dark red color, when blooming.

\subsection{Salt polygons as instability in a dynamical system}

Salt deserts are composed of a bed of unconsolidated gravel, sand, silt and clay, saturated with saline water and overlain by a stable salt crust [4]. Water is replenished from the groundwater with a low background salt concentration and moves upwards through the porous soil [41]. The water evaporates at a high rate through the surface, due to the arid desert climate $[42,43,44,45]$. Salt cannot leave the surface, accumulates and precipitates as a continuous, $2-20 \mathrm{~cm}$ thick crust after reaching maximum saturation in the water. This situation is mainly characterized by the properties of the porous medium, namely its permeability and porosity, exercising drag on the advecting fluid. In addition, the diffusivity of the salt and the evaporation rate and gravity, as the driving forces of the system, influence the dynamics.
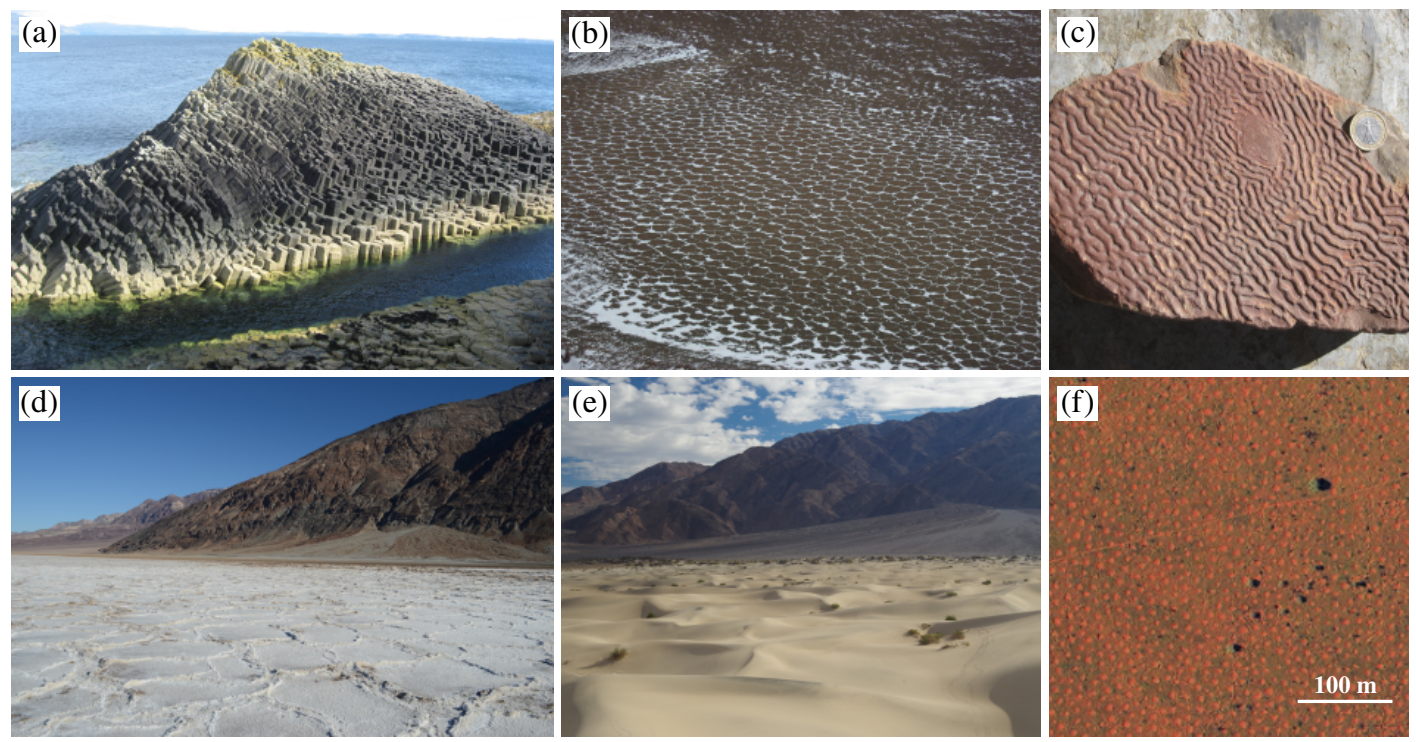

FIGURE 1.8: Examples of patterns found in nature which are the result of an instability in a dynamical process. (a) columnar joints, (b) ice-wedge polygons, (c) Kinneyia fossil mat, (d) salt polygons, (e) dunes and (f) fairy circles. Images (a), (b) and (c) courtesy Lucas Goehring. Image (f) adapted from [46] and courtesy Stephan Getzin. 
Over the course of the following Sec. 1.3.1, I will model the pattern formation phenomenon in salt playa as the result of an instability in a dynamical system [47]. This approach joins the ranks of recent and successful attempts to model pattern formation processes in nature. Prominent examples of these patterns are columnar joints in volcanic rocks [48], as shown in Fig. 1.8 (a) and ice-wedge polygons [49], (Fig. 1.8 panel (b)). Salt polygons from the Badwater Basin at the Death Valley are shown in Fig. 1.8 panel (d). Additional examples are Kinneyia fossils [50] shown in Fig. 1.8 panel (c), sand dunes [51] shown in Fig. 1.8 panel (e) and fairy circles from the Savannas of Africa and Australia [52, 46], Fig. 1.8 panel (f).

Common to all these processes is their dependence on time, their robustness to changes in system parameters and their inherent and characteristic length scale. In the past, there have been attempts to explain the emergence of salt polygons either as cracks $[53,54]$ or wrinkles $[55,56]$. Both approaches only consider the dynamics of the crust itself and not the soil below it, and both fail to explain the intrinsic length scale of the patterns: for both wrinkling and cracking of a sheet that is much thinner than its horizontal extent, the wavelength of the wrinkles and cracks is expected to have the same order of magnitude as the sheet's thickness [57, 48]. Since in nature the crust has a thickness of $2-20 \mathrm{~cm}$ and a polygon diameter of $1-2 \mathrm{~m}$, there is an inexplicable difference of one or more order of magnitude. Moreover, the existing approaches cannot explain the remarkably robust selection of a very narrow range of pattern wavelengths.

On the other hand, there have been numerous studies focusing on density driven brine convection below a salt lake, for example [58, 59, 60]. While successful in modeling the advection and diffusion of the saline water driven by evaporation, these studies have so far neglected any connection to the patterns visible on the surface.

With my approach I combine the existing tools for the description and analysis of dynamical systems with the methodologies from geomorphology, which describe the properties of landscapes and soil surfaces: I explain a phenomenon, which has previously been perceived and described as a pure surface phenomenon, by means of a model that includes both surface and subsurface dynamics. The physics of the underlying model are subject to an instability which depends on the system parameters.

\subsubsection{From description to model}

In developing a model for how surface salt patterns couple to and are driven by subsurface flows, I first consider how those flows may evolve. The dynamics can be described by a model for liquid and salt transport in a porous medium, which is driven by the liquid loss through the surface, and is constantly fed by water with a background salt concentration from below. This system builds up a concentration gradient below the surface as evaporation continues. The stratified liquid can 
become unstable under the right conditions $[58,59,41]$ and exhibit convective dynamics. This model of an evaporating salt pan, including subsurface dynamics and overlying crust, is visualized in Fig. 1.9.

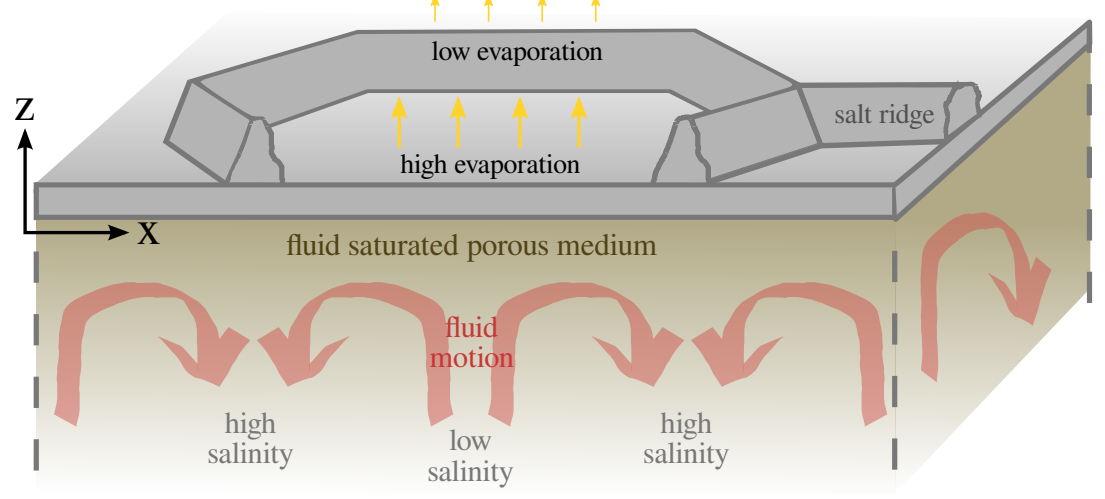

FIGURE 1.9: Illustration of the advection-diffusion dynamics below the salt polygons. Large red arrows indicate underground liquid movement whereas yellow arrows indicate evaporation through the surface. Downwellings are co-localized with salt ridges at the surface.

In Chapter 2 I will develop the equations for fluid and solute transport which corresponds to this simple model, starting with the momentum balance of the fluid, then introducing the porous medium and finally coupling the fluid flow to the motion of the dissolved salt. I list and motivate the assumptions and approximations I make, and describe the boundary conditions I use. The set of boundary conditions, assumptions and approximations sets the stage for an analytical consideration of the salt-pan problem.

Once I lay out the equations governing the dynamics of the system I will define the Rayleigh number, the nondimensional group that scales the equations. Above a critical Rayleigh number, the system will give rise to convective dynamics with a specific wavelength, which couple to the surface and drive the growth of patterns.

\subsubsection{Existing approaches}

Existing research on porous media flow provides a rich set of literature on different types of its dynamics, which can occur in buoyancy driven convection in a porous medium. A very early application of convection models to geophysical applications is the description of the dynamics in geysers by [61]. The theory of convection in a porous medium was then developed further by [62] and [63] who extended the classical theory of Rayleigh-Bénard convection to a porous medium. The theory was soon complemented by experiments by $[64,65]$. Wooding was the first who applied this model to convection driven by salinity gradients rather than temperature gradients [66] and then used it to model the dynamics below an evaporating salt lake [41, 60]. 
Different geological applications warrant slightly different assumptions and boundary conditions. A good and relatively recent review of the different existing approaches and the modern state of modeling is given by $[67,68]$. The main differences in the models used in literature can be summarized into six areas. Below I give a short description of the differences found in literature as well as some exemplary references illustrating the existing work.

- Fluid: The fluid is either composed of two [69, 70] or more [71] miscible (for example pure water and water with a maximum salt concentration, i.e. brine) or immiscible [72] parts (for example water and oil) with different densities and/or viscosities. These works describe and quantify viscous fingering at the interface between two fluids.

- Boundary conditions: Boundaries are either permeable [73] or impermeable [74] to the fluid and temperature / dissolved quantity and boundary conditions are either homogeneous or inhomogeneous in space. Very prominent among this work is the application to the diffusion of $\mathrm{CO}_{2}$ from above into a porous aquifer and the ensuing convective dynamics of the mixture of saline sea water and $\mathrm{CO}_{2}[75,76,77,78,79,80,81,82,83,84,85]$.

- Flow: Density gradients are either caused by dissolution of a quantity (salt / temperature) into the fluid from one boundary of the domain [77, 85] or by constant through-flow of the fluid through the domain, leaving behind the dissolved quantity and building up a density gradient [86, 87]. Systems with a constant dissolution from the top are often found in $\mathrm{CO}_{2}$ sequestration literature or the modeling of waste seepage below landfills [88], whereas constant through flow is used to model the dynamics in saline lakes.

- Forcing: The dynamics are either subject to external forcing by gravity [58, 89] ("vertical" flows such as convection below a salt lake) and/or by pressure [90, 91] ("horizontal" flows such as fluid injection). The influence of gravity on the dynamics of the system was studied before by tilting the experimental setup [75].

- Porous medium: The porous medium is either assumed to be homogeneous (nearly all studies) or inhomogeneous [92, 93, 94] and saturated [95] or unsaturated [96] with fluid. Inhomogeneous porous media resemble the natural conditions in sandstone more closely and are investigated mostly in the $\mathrm{CO}_{2}$ sequestration literature, whereas the investigation of the evaporation from unsaturated porous media is for example interesting to quantify overall fluid loss from soil [97].

- Chemistry: Chemical interactions between the fluid components in the system are either modeled [98] or neglected (nearly all studies). However, chemical interactions are of great importance for the study of degradation of building 
materials such as sandstone [99] or in the investigation of toxic chemicals in the leachate of landfills and catalytic reactions [100].

- Dimensionality: The dynamics can either be modeled in two (nearly all studies) or three dimensions [101, 102, 103]. Often, two-dimensional representations are preferred, because of the computational cost of a full three-dimensional model of the system. However, especially in the $\mathrm{CO}_{2}$ sequestration literature, three dimensional descriptions have been becoming more common, to allow for a description of inhomogeneities in the porous medium and engineering applications.

In principle, flows in a porous medium could be modeled as flows through each individual pore and channel of the medium [104]. Nevertheless, this requires incorporation of the very complex geometry of the medium and is not always necessary to recover quantities such as the overall mass or temperature transport or the velocity and spacing of convective flows. Because of this, continuum approaches in modelling the mass (when a dissolved quantity is concerned), momentum and energy (when temperature is concerned) transport in the system have been predominantly used in the past [105], and will also be used in this work.

In this work I will employ an approach to model convection of two miscible fluids driven by a density gradient caused by a dissolved quantity with external forcing by gravity. The top boundary is permeable to fluid and impermeable to salt as well as inhomogeneous in space. I consider the porous medium to be homogeneous and the domain to be two-dimensional. Last but but not least, I do not consider any chemical reactions inside the fluid or between fluid and porous medium.

\subsection{Predictions}

The advection-diffusion based model of the salt playa system makes several predictions about observables, which can be measured both in the experiment and the field:

- Instability: If the system is unstable and therefore prone to convective dynamics, the Rayleigh number in the experimental and field setting will be above the critical Rayleigh number. The Rayleigh number is calculated from a few parameters, which can be measured in the field and experiment.

- Density stratification: If the system is indeed driven by evaporation, a vertical concentration gradient, will build up below the salt crust. This gradient will be detectable both in an experimental replication of the system as well as directly measurable in the field.

- Convection: If the system is unstable and convection occurs, plumes of high salinity will sink downwards from the surface. This will lead to horizontal differences in concentration, which are measurable. 
- Co-location: If the plumes of high salinity in the underground are indeed the driving mechanism of the growth of salt polygons at the surface, they will be co-located with the ridges of the polygons.

- Wavelength: If the mechanism of convection is the driving mechanism of the pattern formation, the length scale of the underground convection predicted by a linear stability analysis of the system will be consistent with the length scale of the patterns.

- Timescale: As a consistency check, the natural timescale $T$ of the system predicted by theory (see Sec. 2.5, Eq. (2.13) will be consistent with the natural growth cycle of salt polygons in the field in between flooding events.

Verifying these predictions will be the focus of the rest of my work described here.

\subsection{Summary}

In this chapter I introduced the importance of salt deserts as a common landform and their impact on both climate-surface and surface-human interactions. Moreover, I illustrated the importance of fascinating natural phenomena such as salt polygons as a driver for scientific progress. I detailed the specific setting and history of one of my field sites, Owens Lake in California, to illustrate the impact of human interference on nature and the resulting natural disaster, which could have been prevented, given a better understanding of nature.

Following the motivation for this work, I introduced my approach to model the pattern formation in salt deserts as the result of an instability in a dynamical system. This approach was already used successfully in the past to explain phenomena such as fairy circles and columnar joints. For my model I combine the description of the underground dynamics with the description of the patterns visible at the surface and argue that the driving mechanism for pattern formation is an advection-diffusion process in the underground.

\subsection{Scope of this thesis}

In Chapter 2, I will derive the governing equations of water and salt transport in a porous medium, starting from the incompressible Navier-Stokes equations. I will then employ a non-dimensionalization to find the single dimensionless group, the Rayleigh number, which scales the system.

In Chapter 3, I will introduce the methods used to verify the predictions I made in Sec. 1.4: In this work I use the numerical methods described in Sec. 3.1 to simulate the governing equations and investigate the scaling of the dynamics as well as possible feedback with the salt crust at the surface. I complement the numerical simulations with experiments in Hele-Shaw cells, described in Sec. 3.2. The experiments allow me to both investigate the dynamics of the system under controlled 
circumstances as well as develop approaches and protocols for the third part of my work, the field study. In Sec. 3.3 I describe the locations I visited during two field campaigns as well as the measurement methods I employed to collect data about the observables that characterize the natural settings in which salt polygons occur.

In Chapter 4 I describe the evidence I collected using the methods described in Chapter 3 to verify each of the predictions made in Sec. 1.4. In Sec. 4.2 I start with a linear stability analysis of the equations derived in Chapter 2. I find a critical Rayleigh number above which the system is prone to become unstable to perturbations. I present evidence that the settings found in my experiments and in nature fall in the critical regime. In Sec. 4.4 I present direct hydrological evidence for the existence of convection driven by differences in salt concentration both in the experiment and the field. I follow with an investigation of the scaling of the pattern wavelength in Sec. 4.5, based on the scaling of the convective dynamics. Subsequently, in Sec. 4.7 I present an analysis of the time scales on which patterns grow, based on direct and indirect observations of ridge growth in the field. Finally, in Sec. 4.6 I introduce the need for a feedback mechanism between subsurface dynamics and crust, which I investigate in numerical simulations.

In Chapter 5, I will discuss the limitations of my methodology and the data I collected as well as provide an analysis of my results in connection to existing literature on the dynamics of porous media flows and salt crystallization on porous media. In Sec. 5.1 I revisit the approximations I made in Chapter 2 and their impact on the results of my work. In Sections 5.2, 5.3 and 5.4 I discuss the limitations of my methods and the reliability of my results and discuss my results from the perspective of the existing literature. In Sec. 5.5 I will discuss, why the patterns observed in nature are hexagons.

In the first part of Chapter 6, Sec. 6.1, I will summarize my main findings. In Sec. 6.2 will give an outlook on possible future simulations, experiments and field studies, which could help to confirm my results and clarify the exact nature of the coupling between underground dynamics and crust as well as a more extended outlook to the ramifications of my findings.

Finally, as this is a highly collaborative work, contributions from Marcel Ernst (theory, numerics) and Birte Thiede (experiments) are summarized as needed at the required points in this thesis. 


\section{Chapter 2}

\section{Theory}

In this chapter, I will derive the governing equations of water and salt transport in a porous medium. I will revisit the description of the natural setting in which the polygonal growth of salt ridges happens in nature and derive a set of initial and boundary conditions for the mathematical description of the model. I will employ a non-dimensionalization of the governing equations and arrive at the Rayleigh number, a nondimensional group which scales the system. I will also derive the steady state of the salinity distribution in the system, which allows me to compare the theoretical prediction for the salinity distribution to the salinity distributions found in nature. The equations derived in this chapter set the stage for the linear stability analysis and subsequent derivation of the neutral stability curve and most unstable mode of perturbation growth, which I describe in Sec. 4.2.1.

\subsection{Momentum balance of fluid flow}

I start my analytical considerations with the dynamics of a viscous fluid. I assume that the fluid is a continuous quantity and introduce an infinitesimally small representative fluid element of dimensions $d x, d y$ and $d z$ and volume $V$. To arrive at the mass of a volume element, the volume is multiplied with the fluid's density $\rho$.

Since the liquid involved in the dynamics is water at modest pressures, I can safely assume incompressibility. The fluid motion is represented by the temporal evolution of a vector field describing the velocity $v$ of the center of gravity of every volume element and a scalar field describing the pressure $p$ acting on the boundaries of every volume element. By applying Newton's equation of momentum conservation to a continuous quantity, I arrive at the momentum balance equation for a fluid with external forcing $f$

$$
\rho\left(\frac{\partial v}{\partial t}+(v \cdot \nabla) v\right)=\mu \nabla^{2} v-\nabla p+f,
$$

with the incompressibility condition

$$
\nabla \cdot v=0
$$


Here $\mu$ is the dynamic viscosity of the fluid. Eq. (2.1) is the incompressible NavierStokes Eq. [106], which describes advection and diffusion of fluid momentum. In this work, the external forcing is always a buoyancy term in the form of gravity $g=g_{z} \hat{z}$, acting on the mass of every volume element in the negative $z$-direction.

The dimensionless group characterizing fluid dynamics is the Reynolds number [106]. It describes the ratio of inertial to viscous forces in the system

$$
\operatorname{Re}=\frac{\rho v L}{\mu}
$$

where $L$ is the characteristic linear dimension of the domain of fluid flow. In the case of a salt pan, fluid flows through sand, which I model as a stationary porous matrix. In this case, the diameter of the pores of the porous matrix sets the length scale $L$.

For typical values of $v$ and $L$ found in salt pans, I find values of $\operatorname{Re}=\mathcal{O}\left(10^{-7}\right)$, well below the typically assumed value for turbulent flow at $\operatorname{Re} \sim \mathcal{O}\left(10^{3}\right)$. The model therefore describes a "creeping" or Stokes-flow where inertial forces are negligible compared to viscous forces. If I neglect the inertial terms on the left hand side of Eq. (2.1), the momentum balance of the fluid reduces to the Stokes equation

$$
\mu \nabla^{2} v-\nabla p+\rho g=0
$$

\subsection{Flow in a porous medium}

If the fluid moves through a porous medium, every volume element is the sum of the volume occupied by fluid $V_{f}$ and the volume occupied by solid $V_{s}$ and therefore $V=V_{f}+V_{s}$, as illustrated in Fig. 2.1. The porosity of the porous matrix is defined as ratio between the volume occupied by liquid and the total volume of the volume element $\phi=V_{f} / V$.

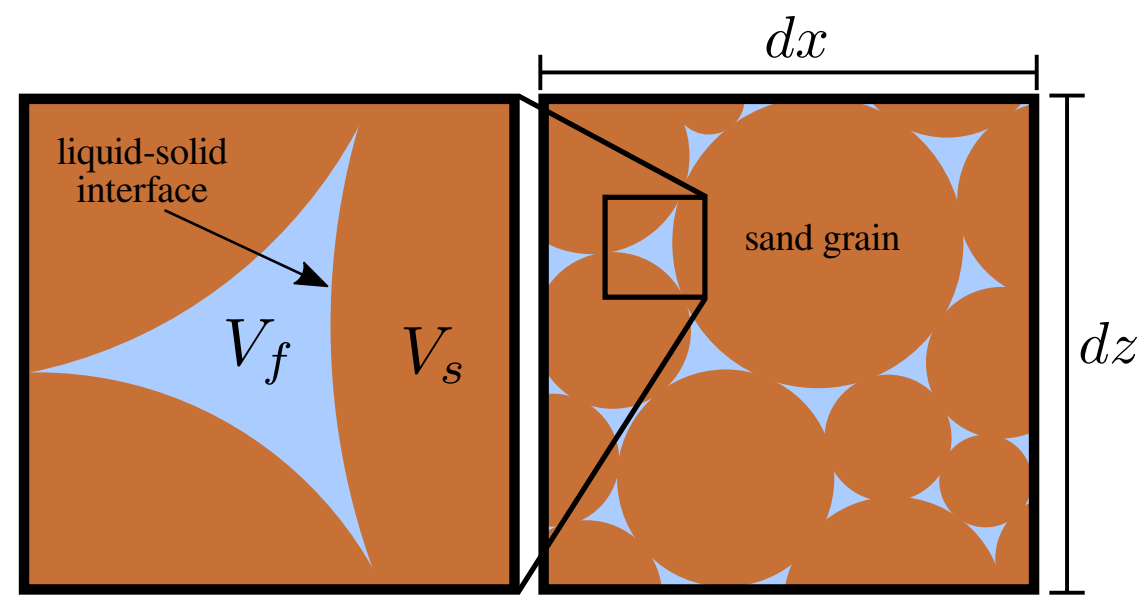

FIGURE 2.1: Schematic representation of a volume $V$ of porous medium with volume $V_{s}$ occupied by the solid matrix and volume $V_{f}$ occupied by the fluid.

For flow through a porous medium, next to the inertial forces, shear forces need to be considered. Inside the fluid, shear forces cancel out between every two volume 
elements but at the interface between the fluid and the porous matrix, shear forces are non-zero. The pore size of the porous matrix is of order $10^{-5} \mathrm{~m}$. I assume, that the volume element $V$ is large with respect to the pore size but still small with respect to the bulk flow. Furthermore I assume, that solid-liquid interfaces are distributed uniformly within the volume element.

The shear stress in each volume element is proportional to the velocity gradient of the fluid and its viscosity. The magnitude of the resulting shear force is the product of the shear stress and the area of the fluid-solid interface. I introduce the permeability $\kappa$ of the porous medium, which is proportional to the area of the fluid-solid interface and can be interpreted as "drag coefficient". I assume the permeability to be isotropic and constant throughout the porous medium, therefore $\kappa$ is a scalar. I can now introduce an additional sink term $-\frac{\mu}{\kappa} v$ for the fluid's momentum when moving through a porous medium. Additionally, I absorb the porosity $\phi$ into the velocity and describe the fluid's motion by a flux (i.e. discharge per unit area) $q=\phi v$ instead of a velocity. Inserting the additional sink term into Eq. (2.3) and substituting $v \phi \rightarrow \boldsymbol{q}$, I arrive at the Darcy-Brinkman-Stokes equation

$$
-\nabla p+\mu \nabla^{2} \boldsymbol{q}-\frac{\mu}{\kappa} \boldsymbol{q}+\rho g=0 .
$$

A more detailed version of above derivation can be found for example at [107] or [105].

Since a porous medium has a very large surface, drag forces become very large as well and dominate over the viscous term $\mu \nabla^{2} q$. By neglecting the viscous term in Eq. (2.4), I arrive at the Darcy equation for flow in a porous medium

$$
\boldsymbol{q}=-\frac{\kappa}{\mu}(\nabla p-\rho \boldsymbol{g})
$$

where $g=-g_{z} \hat{z}$ and $\hat{z}$ is the unit vector in positive z-direction.

\subsection{Coupling to a dissolved quantity}

Ground water in salt deserts has accumulated minerals over centuries and has a substantial salt concentration [4]. I describe the amount of salt which is dissolved in the water by a scalar mass fraction $c_{m}$ of salt, averaged over a volume $V$. The solute couples to the liquid via the liquid's density $\rho=\rho\left(c_{m}\right)$. By including the density in Eq. (2.2), I arrive at the mass balance for the fluid carrying the solute [108]

$$
\phi \frac{\partial \rho}{\partial t}+\nabla \cdot(\rho \boldsymbol{q})=0
$$

I can describe the salty water as the mixture between two liquids: fresh water with density $\rho_{0}$ and water with maximum salt concentration and density $\rho_{s}$. For a mixture of fresh water with brine, I can assume with reasonable accuracy that the two 
volumes behave additively [109] the two liquids behave additively. Therefore, following [108], the volume fraction of concentrated salt solution is given as

$$
c_{v}=c_{m} \frac{\rho}{\rho_{s}}
$$

and the density of the mixture of the two liquids follows

$$
\rho=\left(1-c_{v}\right) \rho_{0}+c_{v} \rho_{s}
$$

The density of the fluid depends on the concentration of solute. The viscosity $\mu=\mu(\rho)$ in turn depends on the density. For water with dissolved salt, this effect is significant and viscosity changes by a factor of about two [109] between pure water and brine with maximum salt concentration. The impact of including the density dependence of the viscosity into the modeling was investigated by [96], who found quantitative differences in the long-term behavior of the model when compared to constant viscosity. Here I choose to neglect the density dependence and take $\mu(\rho)=\mu\left(\rho_{0}\right)=\mu$ to render the problem analytically tractable. Nevertheless, the proportionality between increasing density and viscosity has to be kept in mind and is discussed in Sec. 5.5, since it will become important when the selection of convection planforms is concerned.

Assuming the system is fed by deep groundwater with a given background salt concentration and therefore background density $\rho_{b}$, I can introduce the salinity

$$
S:=\frac{\rho-\rho_{b}}{\rho_{s}-\rho_{b}}, \quad S=[0,1] .
$$

The salinity is subject to advection by the moving fluid $\nabla \cdot(S \boldsymbol{q})$ as well as diffusion, following Fick's second law

$$
\frac{\partial S}{\partial t}=D \nabla^{2} S
$$

Here the diffusion coefficient $\boldsymbol{D}$ is a tensor, which includes molecular diffusivity as well as dispersive contributions due to non-optimal flow patterns. I choose to neglect the velocity dependent contribution of dispersion and use a scalar diffusion coefficient $D$, which I take to be the molecular diffusivity of the dissolved ions. I argue that on the velocity scales I care most about - i.e. the circumstances found in the field - velocities are small enough to warrant this choice. A more thorough discussion of this assumption for the experimental and field settings can be found in Sec. 5.1.1. 
Following [108], I can express the salt conservation as balance between the advective and diffusive flux of salt in the system

$$
\phi \frac{\partial S}{\partial t}+\nabla \cdot(S q)=D \phi \nabla \cdot\left(\frac{\rho_{0}}{\rho} \nabla S\right)
$$

For more details of the derivation, see [110].

\subsection{Coupled equations for fluid and salt transport in a porous medium}

Given the assumptions and approximations described in Sec. 2.2 to 2.1, I arrive at the following set of equations, describing the momentum conservation from Eq. (2.6), salt conservation from Eq. (2.9) and Darcy flow from Eq. (2.5):

$$
\begin{aligned}
\phi \frac{\partial \rho}{\partial t}+\nabla \cdot(\rho \boldsymbol{q}) & =0 \\
\phi \frac{\partial S}{\partial t}+\nabla \cdot(S \boldsymbol{q}) & =D \phi \boldsymbol{\nabla} \cdot\left(\frac{\rho_{0}}{\rho} \boldsymbol{\nabla} S\right) \\
\boldsymbol{q} & =-\frac{\kappa}{\mu} \nabla p+\frac{\kappa \boldsymbol{g}}{\mu} \rho .
\end{aligned}
$$

The system of equations is closed by the dependence of the density on salinity, formulated in Eq. (2.8)

$$
\rho=S \Delta \rho+\rho_{b}
$$

I assume, that differences in inertia due to different densities are negligible. Density differences are only important where the gravity acts on the fluid and the specific weight of a volume element is of importance. This is called Boussinesq-approximation and motivated for example by [111]. Therefore I consider the density only in the external forcing term, where it is multiplied with the acceleration due to gravity and neglect it everywhere else. I absorb the background density $\rho_{b}$ into the pressure term, using the vertical component of the gravity $g_{z}$. Inserting the relation $\rho=S \Delta \rho+\rho_{b}$ from Eq. (2.8), the governing equations reduce to

$$
\begin{aligned}
\boldsymbol{\nabla} \cdot \boldsymbol{q} & =0 \\
\phi \frac{\partial S}{\partial t}+\boldsymbol{q} \cdot \nabla S & =D \phi \nabla^{2} S \\
\boldsymbol{q} & =-\frac{\kappa}{\mu} \boldsymbol{\nabla}\left(p+\rho_{b} g_{z} \widehat{\boldsymbol{z}}\right)+\frac{\kappa \boldsymbol{g}}{\mu} S \Delta \rho .
\end{aligned}
$$

\subsection{Non-dimensionalization}

So far, I have not considered the liquid loss through the surface due to evaporation with rate $E$. The evaporation rate enters the governing equations only through the 
boundary conditions. Nevertheless, in the following I use $E$ to arrive at a dimensionless representation of the governing equations. I take $E$ as a natural choice for the speed of the system and, using the diffusion coefficient $D$, set the natural length and timescale of the system as

$$
L=\frac{D}{E}, \quad T=\frac{D}{E^{2}} .
$$

$L$ can be interpreted as the distance over which advective and diffusive transport have the same order of magnitude, whereas $T$ is the time the flow needs to travel a distance $L$ in my system. For reference, inserting moderate values for $E$ and $D$ found in the field, I find $L \approx 0.3 \mathrm{~m}$ and $T \approx 675$ days for Owens Lake (see Sec. 4.5.4 for details).

I introduce the dimensionless time $\tau=t / T$ as well as space $(X, Y, Z)=\frac{1}{L}(x, y, z)$. Furthermore, I define the dimensionless pressure $P$ which takes into account the term for the absorbed background density first used in Eq. (2.12), and the dimensionless fluid flux

$$
P:=\frac{\kappa}{\phi \mu D}\left(p+\rho_{b} g_{z} \hat{z}\right), \quad \boldsymbol{U}:=\frac{1}{\phi E} \boldsymbol{q} .
$$

Replacing $q \rightarrow U, p \rightarrow P, t \rightarrow \tau$ and $z \rightarrow Z$ in equations (2.10) to (2.12), I arrive at the non-dimensional set of governing equations

$$
\begin{aligned}
\nabla \cdot U & =0 \\
U & =-\nabla P-\operatorname{Ra} S \widehat{Z} \\
\frac{\partial S}{\partial \tau}+\boldsymbol{U} \cdot \nabla S & =\nabla^{2} S .
\end{aligned}
$$

This system of equations is scaled by the dimensionless Rayleigh number

$$
\operatorname{Ra}:=\frac{\kappa \Delta \rho g}{\phi \mu E} .
$$

Ra absorbs the permeability $\kappa$, the density difference $\Delta \rho=\rho_{s}-\rho_{b}$, the gravitational acceleration $g$, the porosity $\phi$, the viscosity $\mu$ and the evaporation rate $E$. The Rayleigh number can be interpreted as the ratio between buoyancy and dissipation in the system. As salinity accumulates at the top boundary of the system, the density difference $\Delta \rho$ increases and with it $\mathrm{Ra}$. If $\mathrm{Ra}$ surpasses a critical value $\mathrm{Ra}_{c}$ and buoyancy effects cannot be counteracted by dissipative forces anymore, the system of stratified salty water becomes unstable and convective motion starts. In Sec. 4.2.1, I will give a quantitative analysis of the onset and nature of this instability as well as the scaling of the convective dynamics with Ra after the system has become unstable. 


\subsection{Boundary Conditions}

The boundary conditions I choose for the system must reflect the conditions under which salt crusts form and express polygonal patterns in nature. In the following, I will motivate the boundary conditions I chose based on my knowledge about the natural conditions that surround pattern emergence.

Salt pans are subject to dramatic inundation events that cover the salt pan, dissolve the patterns and reset the stage for the formation of new patterns after the water has evaporated. While these events are not documented for all areas of the world in which salt ridges occur, they are subject of public weather recordings for Owens Lake and Badwater Basin. Both Badwater Basin as well as Owens lake are subject to rare but very heavy rainfall events in winter and spring, which either directly precipitate on the salt pan, or on the surrounding mountains. The runoff of rain or snowmelt from the mountains is enough to cover the pan with several centimeters to a meter of water.

For Owens lake, artificial flooding brought about by human management efforts also plays a role. During my visits at Owens Lake, I saw several sites which were in the process of being flooded. In one instance, I conducted measurements while the site was being flooded and had to leave prematurely, because of the invading water.

After my second field study, I left three cameras at Owens Lake to record images of the growing crust from January to July 2018 (for details see 3.3.2). One of the cameras captured a flooding event and subsequent formation of crust and ridges. Evidence of this process is displayed in Fig. 2.2. The full timelapse video is provided as supplementary material to this work and available upon request.

After a flooding event, water evaporates completely over the course of a couple of months if conditions are hot and dry. Therefore the water usually accumulates in winter and early spring, as snow starts to melt in the mountains and evaporates until summer. The main growing season for polygons is from April to late summer. After all the water has evaporated, a fresh salt crust quickly forms and ridges start growing. After a time period of 3-4 months, the crust shows pronounced ridges.

In nature, there are four scenarios relevant for modeling of the boundary conditions:

(1) A freely evaporating pool of concentrated brine on top of a permeable soil. This scenario is likely to be found after a flooding event.

(2) Permeable soil saturated with salty water with homogeneous evaporation at the surface. This scenario is likely to be found after the brine pool from scenario (1) has completely evaporated but no salt crust has formed yet. It is a transient scenario that will not persist for long, if evaporation continues.

(3) Permeable soil saturated with salty water and covered with a uniform salt crust and homogeneous evaporation through the crust. This scenario is the 
logical consequence of scenario (2). If evaporation continues and salt continues to accumulate at the surface, it will eventually crystallize on top of the sand.

(4) Permeable soil saturated with salty water and covered with a non-uniform salt crust that modulates evaporation. Salt crusts that have expressed a ridgepattern are likely to fall under this scenario.
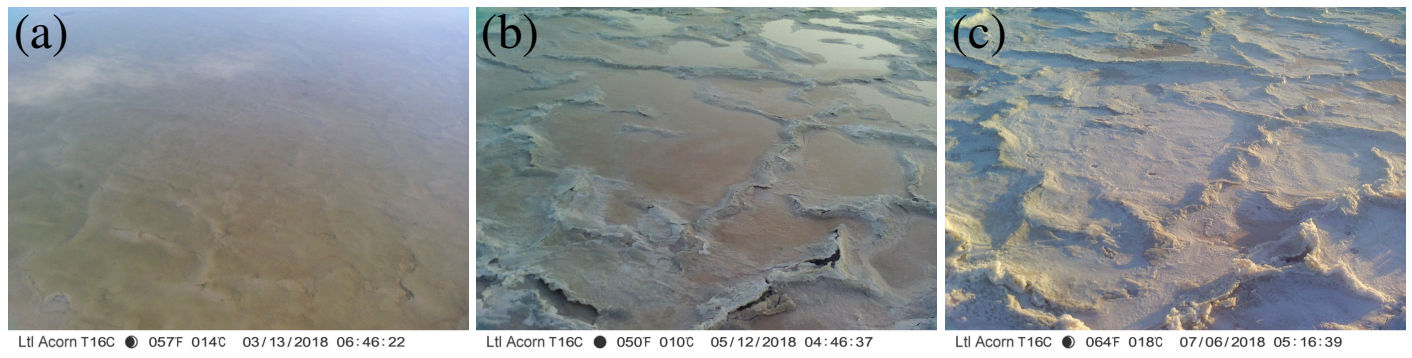

FIGURE 2.2: Images recorded in the first half of 2018 by a camera installed at Owens Lake. (a) the image from March $13^{\text {th }}$ shows the flooded surface corresponding to scenario (1). In this case the underlying crust has not completely dissolved. (b) the image from May $12^{\text {th }}$ shows that the brine has completely evaporated, leaving behind a skeleton crust. (c) the image from July $6^{\text {th }}$ shows the crust with pronounced ridges after significant growth has happened.

\subsubsection{Top boundary}

The main difference of my theoretical approach to the existing literature on convection in porous media, is my choice of boundary conditions for the top boundary. Previous experimental $[78,85,75]$ and numerical $[73,112,113,95]$ work on $\mathrm{CO}_{2}$ sequestration often realizes an impermeable upper boundary and therefore

$$
U_{Z}=0 \quad \text { and } \quad S=1 \quad \text { at } \quad Z=0
$$

with $U_{Z}$ the velocity in vertical direction. This is similar to scenario (1), where pressure along the top boundary is uniform and constant. I call this the "salt lake condition":

$$
P=P_{0} \quad \text { at } \quad Z=0 .
$$

The hallmark and driving force of my model is the evaporation with rate $E$ and therefore liquid loss through the upper boundary of the system. The upper boundary is permeable to liquid but impermeable to salt. Therefore, at the upper boundary I match the vertical velocity to the evaporation rate $E$, which is constant for scenarios (2) and (3):

$$
U_{Z}=\frac{q_{Z}}{\phi E}=1 \quad \text { at } \quad Z=0
$$


For scenario (4), the evaporation rate is not uniform anymore but rather modulated by the presence of ridges. I model this by setting

$$
U_{Z}=\frac{q_{Z}}{E(X)}
$$

with

$$
E(X)=E_{0}\left[1+A_{\mathrm{mod}} \cdot \cos \left(2 \pi \frac{X}{\lambda_{\mathrm{mod}}}\right)\right], \quad A_{\mathrm{mod}}=[0,1]
$$

where $\lambda_{\text {mod }}$ and $A_{\text {mod }}$ are the wavelength and amplitude of the modulation respectively. I do not treat scenario (4) analytically but explore it within the scope of the numerical simulations that will be described later.

\subsubsection{Steady state of the salinity distribution}

In scenarios (1) and (2), the upward advective flux of salinity is balanced by the downward diffusive flux until salinity reaches maximum at $S=1$ :

$$
S=\frac{\partial S}{\partial Z}
$$

In the presence of a precipitated salt crust, as in scenarios (3) and (4), I set the salinity to maximum and impose the boundary condition

$$
S=1 \quad \text { at } \quad Z=0 .
$$

In the steady state, with neither disturbance nor convective dynamics in the system, the salt conservation following Eq. 2.17 is described as

$$
\frac{\partial S}{\partial \tau}+\frac{\partial S}{\partial Z}=\frac{\partial^{2} S}{\partial Z^{2}}
$$

This problem was solved by [41] for times $0 \leq \tau \leq \tau_{\text {saturation. If the salinity fulfills }}$ the boundary condition $S=1$ at saturation time, the steady state solution for the salinity at $\tau_{\text {saturation }}$ is an exponential decay

$$
S^{0}(Z \leq 0)=e^{Z}=e^{\frac{z}{L}}
$$

\subsubsection{Other boundaries}

Since salt pans usually stretch for several hundreds of meters up to tens of kilometers, it is warranted to assume that the system extends infinitely far in the $X$ - and $Y$-direction. To simplify the numerical simulations later on, I choose to employ periodic boundary conditions in this direction.

The soil beneath the salt crust is sandy, unconsolidated sediment reaching downwards many hundreds of meters [114], which is large compared to the bulk flow I 
expect to drive the pattern formation. For a more detailed description of the field setting, see Sec. 3.3.5. This warrants the assumption of an infinite extent of the system in downwards Z-direction, where modelling of the near-surface dynamics is concerned. Therefore I assume

$$
\rho \rightarrow \rho_{b} \quad \text { and } \quad S \rightarrow 0 \quad \text { for } \quad Z \rightarrow-\infty .
$$

\subsection{Summary}

I derive the governing equations of water and salt transport in a porous medium and perform a non-dimensionalization, which allows me to scale the system using only one dimensionless number: the Rayleigh number. The Rayleigh number absorbs all system parameters which are the porosity, permeability, density difference, viscosity, gravity and evaporation rate. Last but not least, I derive the proper boundary conditions of the model from my observations of the natural phenomenon in the field. The derivation of the governing equations allows for an analytic investigation of the conditions for instability in the system, which gives rise to convective dynamics. Moreover, I can employ a numerical simulation of the equations which include the nonlinear terms and allow me to investigate the behavior and scaling of the system with different Rayleigh numbers and boundary conditions. 


\section{Chapter 3}

\section{Materials and Methods}

Pattern formation in salt playa is a complex natural phenomenon. Understanding it requires the use of various methodologies, which are described in this chapter. I approached the problem from many angles to find a conclusive answer to the question of the mechanism driving the pattern formation, therefore the following chapter describes a wide range of methodologies from

- numerical modelling of the dynamics to

- experimental investigation of the dynamics and

- field investigation of the patterns in nature.

In Sec. 1.3 I developed a model of the system of interest and presented an extension of the existing analytical theory of the dynamics. I simplified and modeled the system as an advection-diffusion process in a porous medium based on approaches known from the literature on $\mathrm{CO}_{2}$ sequestration $[67,68]$. Subsequently I recovered the non-dimensional group which scales the system in the form of the Rayleigh number. I also motivated and discussed the key assumptions that underly my modelling of the system.

Based on this theory, in Sec. 3.1 I describe the implementation of a 2D direct numerical simulation of the advection-diffusion dynamics. Work on the numerical model was done in collaboration with Marcel Ernst [110]. I transform the problem into a stream-function-vorticity formulation and implement numerical methods which are well known from the modelling of laminar flows to solve the ensuing Poisson's equation and perform time integration. The simulation enables me to scan the parameters governing the system as well as investigate different boundary conditions which correspond to the situations found in nature.

In parallel I set up quasi-2D laboratory experiments that mirror the simulations and are described in section 3.2. The experiments help me both to validate the simulation as well as to investigate the dynamics at very late times which are inaccessible to the simulations due to computational costs. Additionally I develop measurement protocols for the last and most important part of this work: the investigation of the patterns themselves in nature.

During my two field campaigns I collected direct hydrological evidence of salinity gradients in the sand underneath the hexagonal polygons formed by salt ridges 
protruding from the flat salt crust, and measured the dominant wavelength of the patterns using terrestrial laser scanning. The methodologies used in the field studies as well as a description of the conditions in the field are described in the last Sec. 3.3 of this chapter.

\subsection{Simulation}

The set of non-dimensional governing equations (2.15) to (2.16) can be treated analytically by means of linear stability analysis (as described in Sec. 4.2.1). Nevertheless, my analytical analysis stays limited to linear approximations of the full dynamics. The linear stability analysis is suitable to arrive at accurate predictions for the onset of stability and the expected initial growth rates. Additionally I implement a direct numerical simulation of the governing equations to get information about

- the time development of the system,

- the behaviour of the system away from onset of instability,

- the behaviour of the system away from the top boundary as well as

- the behavior of the system with different boundary conditions at the top.

A direct numerical simulation of the full set of governing equations is also able to capture the nonlinear interactions in the system, that have been neglected in the analytical approach.

The following describes the work of Marcel Ernst and myself (Jana Lasser) who both worked on the implementation of the numerical simulations. The work was divided as follows

- Choice of methodology and initial draft of the simulations: Jana Lasser

- Implementation of simulation and refinement of methodology: Marcel Ernst

- Validation against existing simulations and theory: Marcel Ernst

- Numerical experiments: Jana Lasser

- Documentation: Jana Lasser

In the following, Sec. 3.1.1 and Sections 3.1.3 - 3.1.6 are a summary of a more extensive description of the numerical implementation by Marcel Ernst in his master's thesis [110]. 


\subsubsection{Numerical Approach}

I model the advection-diffusion dynamics as a two-dimensional system with width $W$ in the $X$-direction and depth $H$ in the Z-direction, as depicted in Fig. 3.1. Coordinates are always nondimensional in the following description.

To solve the coupled equations of fluid and salt transport for times $t>0$, I discretize the system and employ standard algorithms for numerical simulations of fluid dynamics, described in more detail below. I transform the equations of motion into a stream-function and vorticity representation, eliminating the pressure. For every time step of the simulation I then solve for the stream function and integrate over time

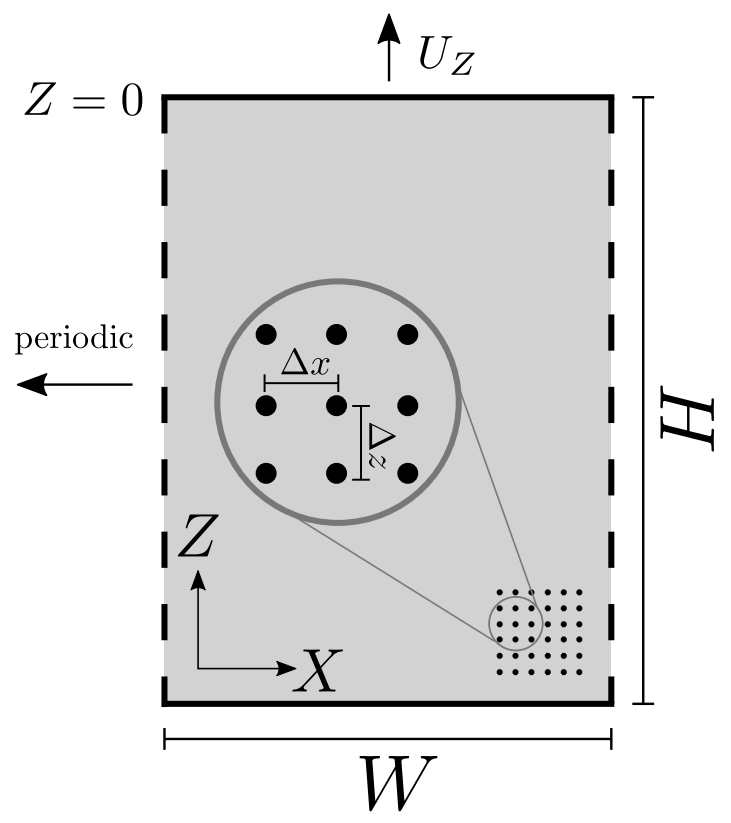

FIGURE 3.1: Illustration of the simulated domain with width $W$, depth $H$ and resolution $\Delta x=\Delta z$. The domain is periodic in the $X$-direction. At the top, the vertical velocity $U_{Z}(Z=0)$ is set. in the following way:

(1) compute the vorticity from the salinity field using a sixth-order compact finite difference scheme

(2) solve Poisson's equation for the stream function employing a semi-spectral Fourier-Galerkin method by

(2.1) transforming Poisson's equation into Fourier space,

(2.2) solving the resulting system of linear differential equations of first order for the stream function by using a Fourier expansion and analytical solution in the $X$-direction and a compact finite difference scheme in the Z-direction and

(2.3) using the backward Fourier transform (using the FFT algorithm) to compute the stream function in real space

(3) compute the new velocity field from the stream function by computing the first order spatial derivatives using a sixth-order compact finite difference scheme

(4) advance the salinity distribution in time by using a third-order Runge-Kutta scheme with adaptive time-stepping

The choice of methodology is based on previous work by [115, 116] and [117], who simulated similar dynamics of a fluid in a porous medium, inspired by applications in $\mathrm{CO}_{2}$ sequestration. 


\subsubsection{Dimensionality}

Our first goal is to understand the scaling of the dynamics underneath the patterns. Given the computational cost of three dimensional simulations and the strictly laminar flow, I resort to simulations of a two-dimensional system. Nevertheless, there have been simulations considering similar systems in three dimensions [118, 103], which are of interest when solute flux and convection cell geometries are concerned. Apart from these simulations on systems with very limited size of about $5 \times 5$ convection cells, two-dimensional modelling of flows in porous media is wide spread throughout the literature.

\subsubsection{Differential Equations}

The system is assumed to be periodic in the $X$-direction. At the bottom and top boundary I assume $S=0$ and maximum salinity $S=1$ respectively, using the definition of the non-dimensional salinity from Eq. (2.8). The average velocity $U_{Z}$ at the top boundary is assumed to be $\left\langle U_{z}(X, Z=0)\right\rangle=1$, reflecting an imposed evaporation rate $E(X)$. This is either accomplished by setting $U_{Z}(X, Z)=1$ everywhere or by imposing a sinusoidal modulation on the velocity in $Z$-direction.

To eliminate the pressure, I follow a stream-function-vorticity approach analogous to $[115,116]$. The relation between the vorticity $\omega(X, Z, \tau)$, stream function $\psi(X, Z, \tau)$ and velocity $\boldsymbol{U}$ is defined as

$$
\begin{gathered}
\omega=\nabla \times \boldsymbol{U}=\frac{\partial U_{Z}}{\partial X}-\frac{\partial U_{X}}{\partial Z} \\
U_{X}=\frac{\partial \psi}{\partial Z} \quad U_{Z}=1-\frac{\partial \psi}{\partial X},
\end{gathered}
$$

and related via Poisson's equation [119]

$$
\nabla^{2} \psi=-\omega
$$

Since $\nabla \times \nabla \Psi=0$ for arbitrary scalar fields $\Psi$, I can eliminate the pressure from Eq. (2.16) by taking the curl

$$
\begin{aligned}
\nabla \times U & =-\nabla \times \nabla P-\nabla \times(\operatorname{Ra} \widehat{Z}) \\
\Rightarrow \omega(X, Z, \tau) & =R a \frac{\partial S(X, Z, \tau)}{\partial X} .
\end{aligned}
$$

Equations (2.17), (3.3) and (3.2) now describe the transport of fluid and salt in terms of the stream function $\psi$, vorticity $\omega$, velocity $U$ and salinity $S$ scaled by the Rayleigh number Ra.

In the case of a non-uniform flow rate at the top boundary, the problem becomes more difficult to solve computationally because singularities might arise at the top boundary. This problem was solved by [120] by splitting the solution of the velocity field into a constant potential component and a rotational component. I absorb the 
constant inhomogeneous terms in $\boldsymbol{U}^{0}(X, Z), \psi^{0}(X, Z)$ and $\omega^{0}(X, Z)$, which fulfill the conditions

$$
\begin{aligned}
\nabla^{2} \psi^{0}(X, Z) & =-\omega^{0}(X, Z) \\
U^{0}(X, Z) & =\frac{\partial \psi^{0}(X, Z)}{\partial Z} \widehat{X}-\frac{\partial \psi^{0}(X, Z)}{\partial X} \widehat{Z}
\end{aligned}
$$

for $\psi(X, Z, \tau)=\psi^{0}(X, Z)+\widetilde{\psi}(X, Z, \tau)$ and analogous for $\omega(X, Z, \tau)$ and $\boldsymbol{U}(X, Z, \tau)$. I insert $\omega(X, Z, \tau)=\omega^{0}(X, Z)+\widetilde{\omega}(X, Z, \tau)$ into Eq. (3.3). From Eq. (3.2) and condition (3.4) follows, that the rotational component also has to follow Poisson's equation. Similarly, from Eq. (3.1) and condition (3.5), a relation for the velocity follows. Last but not least, I use the salt conservation formulated in Eq. (2.17). Collecting the modified differential equations, I arrive at

$$
\begin{aligned}
\widetilde{\omega}(X, Z, \tau) & =\operatorname{Ra} \partial_{X} S(X, Z, \tau)-\omega^{0}(X, Z) \\
\nabla^{2} \widetilde{\psi}(X, Z, \tau) & =-\widetilde{\omega}(X, Z, \tau) \\
\boldsymbol{U}(X, Z, \tau) & =\left[\partial_{Z}\left(\widetilde{\psi}(X, Z, \tau)+\psi^{0}(X, Z)\right)\right] \widehat{X}+\left[1-\partial_{X}\left(\widetilde{\psi}(X, Z, \tau)+\psi^{0}(X, Z)\right)\right] \widehat{Z} \\
\partial_{\tau} S(X, Z, \tau) & =-\boldsymbol{U}(X, Z, \tau) \cdot \nabla S(X, Z, \tau)+\nabla^{2} S(X, Z, \tau)
\end{aligned}
$$

The boundary conditions at the top and bottom for the salinity, stream function, vorticity and velocity in the Z-direction for both the uniform as well as the nonuniform case are listed in Tab. 3.1. The boundary condition in the $X$-direction for any field $\Psi(X, Z)$ at $X=0$ and $X=W$ is periodic, and therefore $\Psi(0, Z)=\Psi(W, Z)$.

\begin{tabular}{c|c|c|c}
\multicolumn{2}{c}{ uniform } & \multicolumn{2}{c}{ non-uniform } \\
$Z=0$ & $Z=-H$ & $Z=0$ & $Z=-H$ \\
\hline$S(X)=1$ & $S(X)=0$ & $S(X)=1$ & $S(X)=0$ \\
$U_{Z}(X)=1-U_{Z}^{0}(X)$ & $U_{Z}(X)=+1$ & $U_{Z}^{0}(X)=1-A_{\bmod } \cdot \cos \left(\frac{2 \pi X}{\lambda_{\bmod }}\right)$ & $U_{Z}^{0}(X)=1$ \\
$\frac{\partial \psi}{\partial X}=0$ & $\frac{\partial \psi}{\partial X}=0$ & $\widetilde{\psi}(X)=0$ & $\omega(X)=0$ \\
$\omega(X)=0$ & $\omega(X)=0$ & $\omega(X)=0$ & $\omega(X)=0$
\end{tabular}

TABLE 3.1: Boundary conditions for the salinity $S$ stream function $\psi$ and vertical velocity $U_{Z}$ in case of a uniform (left) and non-uniform (right) flow rate at the top.

The velocity field from an exemplary implementation of the system with uniform boundary condition is shown in Fig. 3.2 (b). The same simulation but with modulated top boundary condition and its effect on $U_{Z}(X, Z)$ is shown in Fig. 3.3 (c). Equations (3.6) to (3.9) are the equations I am going to solve numerically with the methods described in sections 3.1.4 to 3.1.6 below.

\subsubsection{Compact Finite Difference Scheme}

To numerically compute the first and second derivatives in space of $S(X, Z, \tau)$ and $\psi(X, Z, \tau)$, I employ a sixth order compact finite difference scheme, since it is both 
accurate and very stable [121, 122]. Especially for the Dirichlet boundary conditions applied to my system, the calculation scheme at the boundaries is of the same order of accuracy as away from the boundaries. This is important because the (top) boundary is of crucial importance both for the physically correct representation of the modeled dynamics as well as the numerical stability of the simulation. I use a sixth-order scheme because it represents a good compromise between computational cost and accuracy.

According to [121] and [122], the calculation schemes for the first and second order derivatives for an interior grid point $i$ at position $x_{i}$, where $x_{i}=h \cdot i, i \in \mathbb{N}$, with grid spacing $h$, are given as

$$
\begin{aligned}
f_{i}^{\prime}+\sum_{j=1}^{l_{\alpha}} \alpha_{j}\left(f_{i+j}^{\prime}+f_{i-j}^{\prime}\right) & =\sum_{j=1}^{l_{a}} a_{j} \frac{f_{i+j}-f_{i-j}}{2 j h} \\
f_{i}^{\prime \prime}+\sum_{j=1}^{l_{\beta}} \beta_{j}\left(f_{i+j}^{\prime \prime}+f_{i-j}^{\prime \prime}\right) & =\sum_{j=1}^{l_{b}} b_{j} \frac{f_{i+j}-f_{i}+f_{i-j}}{j^{2} h^{2}}
\end{aligned}
$$

with stencil sizes $l_{\alpha}, l_{a}$ and $l_{\beta}, l_{b}$ as well as constants $a, \alpha$ and $b, \beta$ which depend on the stencil size as well as the desired order (accuracy) of the scheme.

Since I use periodic boundary conditions in X-direction, the above equations can be computed everywhere in the system. For the Dirichlet boundary conditions at the top and bottom of the system, I need to employ an asymmetric calculation scheme. For a grid point at $i=0$, such a scheme is for example

$$
f_{0}^{\prime}+\sum_{j=1}^{l_{\alpha}} \alpha_{j} f_{j}^{\prime}=\frac{1}{h} \sum_{j=1}^{l_{a}} a_{j} f_{j} .
$$

Schemes for grid points $i<$ stencil sizes as well as second order derivatives are computed analogously.

The coefficients $\alpha_{i}, \beta_{i}, a_{i}$ and $b_{i}$ for boundary and interior grid points for first and second order derivatives are listed in [110].

For $N$ grid points $0 \leq i \leq N-1$ I have a system of $N$ linear differential equations which can be written in matrix form as

$$
A_{\text {left }} f^{(n)}=A_{\text {right }} f \Rightarrow f^{(n)}=\left(A_{\text {left }}^{-1} A_{\text {right }}\right) f \text {, }
$$

where $A_{\text {left }}^{-1}$ is the inverted left-hand matrix. Inversion of the matrix is possible because the matrix has a non-zero determinant and needs to be done only once, because coefficients do not change during the course of the simulation. Computation of derivatives therefore amounts to a multiplication of the respective field with the matrix $Q:=A_{\text {left }}^{-1} A_{\text {right }}$. 


\subsubsection{Pseudo-Spectral Approach}

To solve Poisson's Eq. (3.2) for the stream function, I employ a semi-spectral approach in the form of the Fourier-Galerkin method as described in [116, 115, 120, 123]. Since I simulate a system of laminar flow at low Reynolds numbers, solutions are expected to be smooth. Therefore I can resort to computationally cheap Fourier methods while still correctly capturing the physics of the system.

I discretize the system to a grid of $M \times N$ points with spacing $\Delta x$ and $\Delta z$. Subsequently I can define the discretized streamfunction and vorticity $\widetilde{\psi}\left(x_{m}, z_{n}\right)$ and $\widetilde{\omega}\left(x_{m}, z_{n}\right)$ with $x_{m}=m \Delta x, 0 \leq m \leq M-1$ and $z_{n}=n \Delta z, 0 \leq n \leq N-1$. Since the system is periodic in X-direction, I can I perform a Fourier expansion of $\widetilde{\psi}$ and $\widetilde{\omega}$ in that direction:

$$
\begin{aligned}
\widetilde{\psi}\left(x_{m}, z_{n}, \tau\right) & =\sum_{k=-M / 2}^{M / 2-1} \widehat{\psi}_{k}\left(z_{n}, \tau\right) e^{-2 \pi i \frac{x_{m} k}{W}} \\
\widetilde{\omega}\left(x_{m}, z_{n}, \tau\right) & =\sum_{k=-M / 2}^{M / 2-1} \widehat{\omega}_{k}\left(z_{n}, \tau\right) e^{-2 \pi i \frac{x_{m k}}{W}}
\end{aligned}
$$

with Fourier coefficients $\widehat{\psi}_{k}\left(z_{n}, \tau\right)$ and $\widehat{\omega}_{k}\left(z_{n}, \tau\right) . W$ is the extent of the domain in the $X$-direction. Inserting the expansions from equations (3.11) and (3.12) into Poisson's Eq. (3.2) yields

$$
\begin{aligned}
& \frac{\partial^{2} \widetilde{\psi}}{\partial X^{2}}+\frac{\partial^{2} \widetilde{\psi}}{\partial Z^{2}}=-\widetilde{\omega} \\
& \Rightarrow \sum_{k=-M / 2}^{M / 2-1}\left[\left(\partial_{X X}+\partial_{Z Z}\right) \widehat{\psi}_{k}\left(z_{n}, \tau\right) e^{-2 \pi i \frac{x_{m} k}{W}}\right]=\sum_{k=-M / 2}^{M / 2-1}\left[\widehat{\omega}_{k}\left(z_{n}, \tau\right) e^{-2 \pi i \frac{x_{m k}}{W}}\right] \text {. }
\end{aligned}
$$

From this I can compute the second order derivatives in the $X$-direction analytically and the second order derivatives in the Z-direction with the compact finite difference scheme described in Eq. (3.10). I arrive at the linear system of equations for the Fourier coefficients

$$
\widehat{\psi}\left(z_{n}, \tau\right)=-\left[-A_{\text {left }}\left(\frac{2 \pi k}{W}\right)^{2}+A_{\text {right }}\right]^{-1} A_{\text {left }} \widehat{\omega}_{k}\left(z_{n}, \tau\right) .
$$

I can now compute the stream function $\psi\left(x_{m}, z_{n}\right)$ by applying the backward Fourier transform on the coefficients $\widehat{\psi}\left(z_{n}, \tau\right)$. Since the velocity $\boldsymbol{U}$ satisfies the relation

$$
\boldsymbol{U}=\frac{\partial \psi}{\partial Z} \widehat{X}+\left(1-\frac{\partial \psi}{\partial X}\right) \widehat{Z}
$$

I can calculate the velocity field by computing the partial derivatives of the stream function in X-and Z-direction using again a compact finite difference scheme. 


\subsubsection{Time Integration}

I compute the velocity field as described above for every time step $\Delta \tau$. With the known velocity field I am able to advance the salinity $S$ in time according to Eq. (2.17). For this purpose I employ an explicit fourth-order Runge-Kutta scheme with adaptive time-stepping [124]. I rewrite Eq. (2.17) as

$$
\frac{\partial S}{\partial \tau}=: F(\boldsymbol{U}, S)
$$

and construct the updated salinity $S^{(\tau+\Delta \tau)}$ as

$$
S^{(\tau+\Delta \tau)}=S^{(\tau)}+\Delta \tau\left[\alpha F\left(\boldsymbol{U}, S^{(\tau)}\right)+\beta F\left(\boldsymbol{U}, S^{(\tau-\Delta \tau)}\right)\right],
$$

where $S^{(\tau)}$ is the salinity from the last time step and $S^{\tau-\Delta \tau}$ is the salinity from the second to last time step. I use the the coefficients $\alpha$ and $\beta$ given by [124].

\subsubsection{Simulation Parameters}

\section{System size, resolution, duration}

The number of grid points in the $X$ - and Z-directions is given by the system size divided by the resolution: $M=H / \Delta Z$ and $N=W / \Delta X$ respectively. For the sake of simplicity I choose $\Delta X=\Delta Z$ and refer to the spatial resolution as $\Delta X$. I define the aspect ratio $B:=W / H$ where $B=1$ describes a square system.

Since I solve the equations numerically using only explicit schemes, the complexity of the algorithm is proportional to the complexity of matrix multiplications and therefore scales with $\mathcal{O}\left(N^{3}\right)$ for $B=1$. Simulating systems with large numbers of grid points is quite expensive, therefore system sizes cannot be arbitrarily large while keeping the resolution small enough to resolve the physics correctly. The balance between computational cost, system size and resolution is not trivial, especially since I did not implement massively parallelized, GPU-based algorithms due to lack of time, but computed individual simulations on CPUs. In general I was able to simulate systems with a size of $N \times M \sim 3 \cdot 10^{5}$ grid points and simulations took between several days and several weeks.

Some guiding principles to estimate a correct system size and resolution can be found by looking into the size of the system in nature: Polygons are of order $1 \mathrm{~m}$ wide (see Tab. 4.7), while natural length scales $L$ are of order $0.3 \mathrm{~m}$ at moderate evaporation rates. Therefore a simulation of a system large enough to encompass at least three polygons will be expected to require a width of $W \geq 10 \mathrm{~L}$. Since I expect the dynamics in the underground that influence the emergence of polygonal patterns to take place down to a depth of at least one polygon width, I conclude the simulations should have a depth of at least $H \geq 3$, preferably deeper.

As for the resolution $\Delta X$, I had to ensure that my simulation is able to resolve all potentially unstable wavelengths in the system. I use $[125,73]$ as orientation and 
choose a minimum resolution of $\Delta X=1.25 \cdot 10^{-1} \mathrm{~L}$ at $\mathrm{Ra}=20$. For higher Rayleigh numbers I decrease system sizes and increase resolution to up to $\Delta X=1.25 \cdot 10^{-2} \mathrm{~L}$. At $\mathrm{Ra}=2000$, the linear stability analysis (see Sec. 4.2.1) predicts the most unstable wavenumber to be $a \approx 12$ and therefore $\Lambda \approx 0.5 \mathrm{~L}$, which is still 40 times larger than the resolution of the simulation.

Since systems at different Rayleigh numbers express different speeds in the development of their dynamics, I also adapt the time-resolution $\Delta T$ in which I save the system state $\left(S, U_{Z}, U_{X}\right)$ for later analysis. Typical system sizes, resolutions and time-resolutions for the simulations in this work are listed in Tab. 3.2.

\begin{tabular}{c|c|c|c}
$\mathrm{Ra}$ & $H \times W[L]$ & $\Delta X$ & $\Delta T$ \\
\hline $20-38$ & $20 \times 20$ & $1 / 8$ & 0.1 \\
$40-140$ & $100 \times 40$ & $1 / 8$ & 0.1 \\
$160-180$ & $100 \times 40$ & $1 / 8$ & 0.05 \\
$200-280$ & $80 \times 40$ & $1 / 12$ & 0.05 \\
300 & $25 \times 12$ & $1 / 20$ & 0.05 \\
$400-500$ & $20 \times 10$ & $1 / 40$ & 0.01 \\
$600-700$ & $15 \times 7$ & $1 / 60$ & 0.005 \\
$800-2000$ & $10 \times 5$ & $1 / 80$ & 0.001
\end{tabular}

TABLE 3.2: System dimensions $H \times W$ and spatial and time resolutions $\Delta X$ and $\Delta T$ respectively of simulations for different Ra.

I choose simulation durations such that the dynamics of the system, including formation of the initial instability and the coarsening regimes, is captured. This is not trivial to achieve for simulations with either very low or very high Ra. The system shows an exponential slowing of the dynamics as $\mathrm{Ra} \rightarrow \mathrm{Ra}_{c}$, where $\mathrm{Ra}_{c}$ is the critical Rayleigh number for the onset of instability (see Sec. 4.2.1 and Sec. 4.3.1 for details). Therefore the time that plumes take to sink down and coarsen diverges as $\mathrm{Ra}$ approaches $\mathrm{Ra}_{c}$. Simulations have to be stopped at some point and I am not always able to observe the behaviour of the system late at times.

For $\mathrm{Ra}>200$ the dynamics show vigorous convection and the velocity $|\boldsymbol{U}|_{\max }$ tends to be very large. This requires very small time steps $\Delta \tau$ to keep the simulation numerically stable. Therefore simulation of longer times is computationally very expensive. Here too I have to stop simulations at some point and am not always able to observe the behaviour of the system at late times.

The bottom boundary condition I use for the system, i.e. $S=0$ for $Z \rightarrow \infty$ is only physical for an infinite downwards extend of the simulated domain, which is of course not achievable. To prevent information of the unphysical boundary condition from propagating upwards and influencing the dynamics of interest at the top boundary, I stopped the simulations once the first plume impacted the bottom boundary. To have a sufficiently long period of time to observe the dynamics before I had to stop the simulations, vertical extent of the simulations was always chosen to be equal or larger than the horizontal extent. 


\section{Rayleigh Number}

As explained in Sec. 2.3, I assume a scalar diffusion constant $D$ and constant viscosity $\mu$ as well as a homogeneous porous medium resulting in homogeneous and constant permeability $\kappa$ and porosity $\phi$. Overall these assumptions lead to a Rayleigh number Ra that is homogeneous throughout the simulated domain and which characterizes the simulation. The Rayleigh number is assumed to scale the dynamics of the system, therefore it is the main parameter that is varied throughout different simulation runs described in this work.
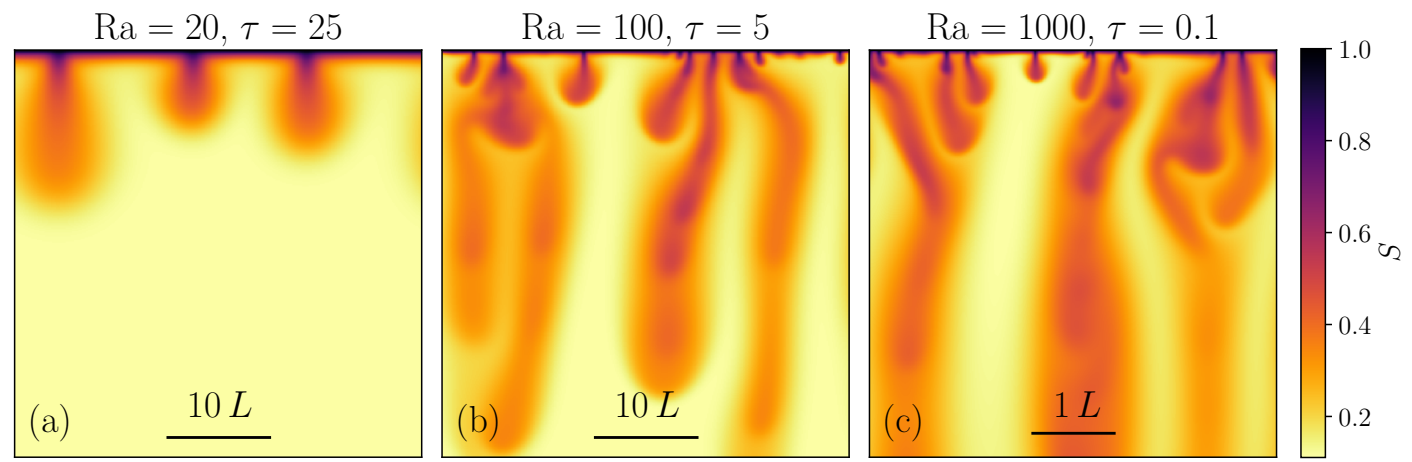

FIGURE 3.2: Exemplary snapshots of simulations for $R a=20,100$ and 1000 at times $\tau_{i}$. Note the different times $\tau_{i}$ and system sizes.

\section{Evaporation modulation wavelength and amplitude}

In simulations where $U_{Z}(Z=0, X)=U_{Z}^{0}=1-a_{\text {mod }} \cdot \cos \left(a_{\bmod } X\right)$, I can vary the amplitude $a_{\text {mod }}$ and scale $a_{\text {mod }}$ of the evaporation rate modulation at the top boundary. To explore if and for how long the downwelling plumes stay aligned with the modulation at the top boundary, I scan these parameters in the range of

- $A_{\text {mod }}=0.001-1.0$,

- $a_{\mathrm{mod}}=0.31-3.76$,

with $U_{Z}^{0}=1$.

\section{Initial conditions}

As an initial condition for the salinity $S(X, Z)$ I start with the steady state solution of Eq. (3.9)

$$
S^{0}(X, Z)=\frac{e^{Z}-e^{-H}}{1-e^{-H}}
$$

I choose this initial condition instead of a sharp drop in salinity below the top boundary to avoid numerical instabilities due to diverging salinity gradients. An exemplary implementation of the initial condition for $S(X, Y)$ for a system of size $40 L \times 40 L$ is displayed in Fig 3.3 (a). 
I seed the steady state salinity distribution with random perturbations $\Delta S$ to break the symmetry of the system and trigger any potential instabilities:

$$
S(X, Z, 0)=S^{0}+\Delta S
$$

Following [116], I introduce perturbations of the form of a field of random numbers $f(X, Z, 0)$, uniformly distributed in the interval $[-1,1]$ and scaled with the amplitude $\gamma=10^{-5}$. I convolve the field of random numbers with an exponential kernel of size $\sigma=3 \Delta X$ to smooth the perturbations. The perturbations $\Delta S$ are then given as

$$
\Delta S(X, Z, 0)=\gamma f(X, Z, 0) * e^{-\frac{X^{2}+Z^{2}}{\sigma^{2}}} .
$$

The perturbation amplitude is small enough to not bias the ensuing dynamics, since at the maximum $\mathrm{Ra}=2000$ simulated in this work it is still four orders of magnitude smaller than the smallest unstable perturbation wavelength. It is also small enough to avoid problems with diverging derivatives, but nevertheless large enough to avoid floating point errors.

Fig. 3.3 (b) shows the velocity field $\boldsymbol{U}(X, Z, \Delta \tau)$ after the first time integration for the case of a uniform evaporation rate at the top boundary. The perturbations are the result of the noise in $S(X, Z, 0)$. Figure. 3.3 (c) shows the velocity field $U(X, Z, \Delta \tau)$ after the first time integration for the case of a modulated evaporation rate at the top boundary.
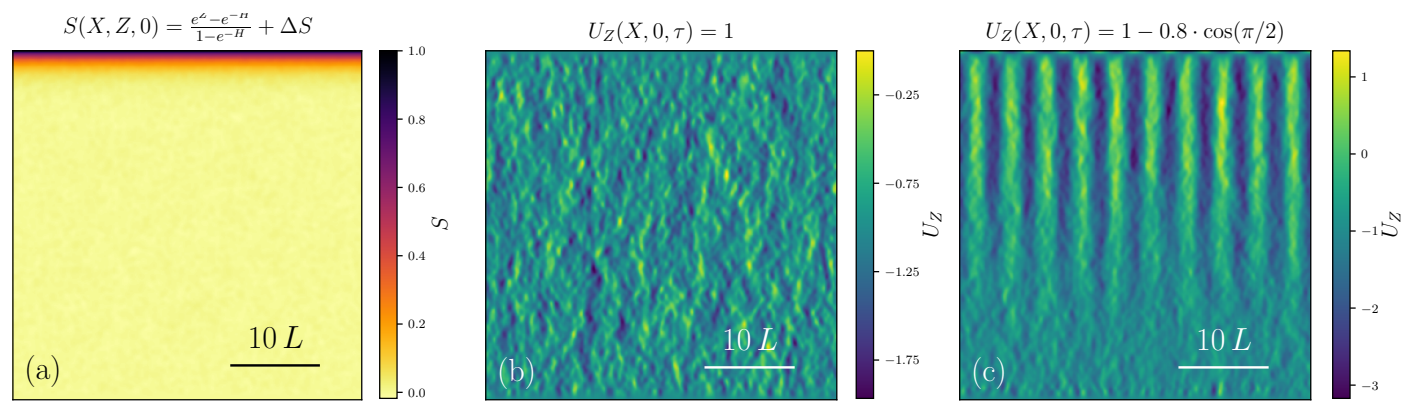

FIGURE 3.3: (a) Initial condition for the salinity $S$. Configuration of $U_{Z}(X, Z)$ at time $\tau=\Delta \tau$ for (b) a uniform top boundary condition and (c) a modulated top boundary condition.

\section{Validation of growth rates}

The numerical simulation code used in this work was validated by [110] by comparing results of the simulations to theoretical predictions. Specifically, he compared the growth rate $\Omega$ (see Sec. 4.2.1 for details) of the initial perturbations to growth rates measured in the simulations and finds very good agreement between the two. 


\subsubsection{Measurements}

\section{Wavelength}

One characteristic property of the system of interest is the number of plumes $N_{p}(Z, \tau)$ at a given depth $Z=Z_{0}$ and point in time $\tau=\tau_{0}$, which - together with the system width $W$ - enables me to compute a dimensionless wavelength $\Lambda=W / N_{p}$ as well as a wavenumber $a=2 \pi / \Lambda$. I count the number of plumes by searching for maxima in the salinity distribution $S\left(X, Z_{0}, \tau_{0}\right)$.

Before I search for maxima, I smooth the salinity field with a uniform kernel of size $3 \Delta X \times 3 \Delta X$ and apply a cutoff at $S=0.1$, to render the search algorithm robust to noise with a small amplitude. Both the smoothing as well as the cutoff operations are important to exclude any additional maxima due to the initial noise in areas farther away from the initial instability which the downwelling plumes have not reached yet. Figure 3.4 shows an exemplary salinity distribution for a system with $R a=100$ at time $\tau_{0}=1.0$ and depth $Z_{0}=-1$.

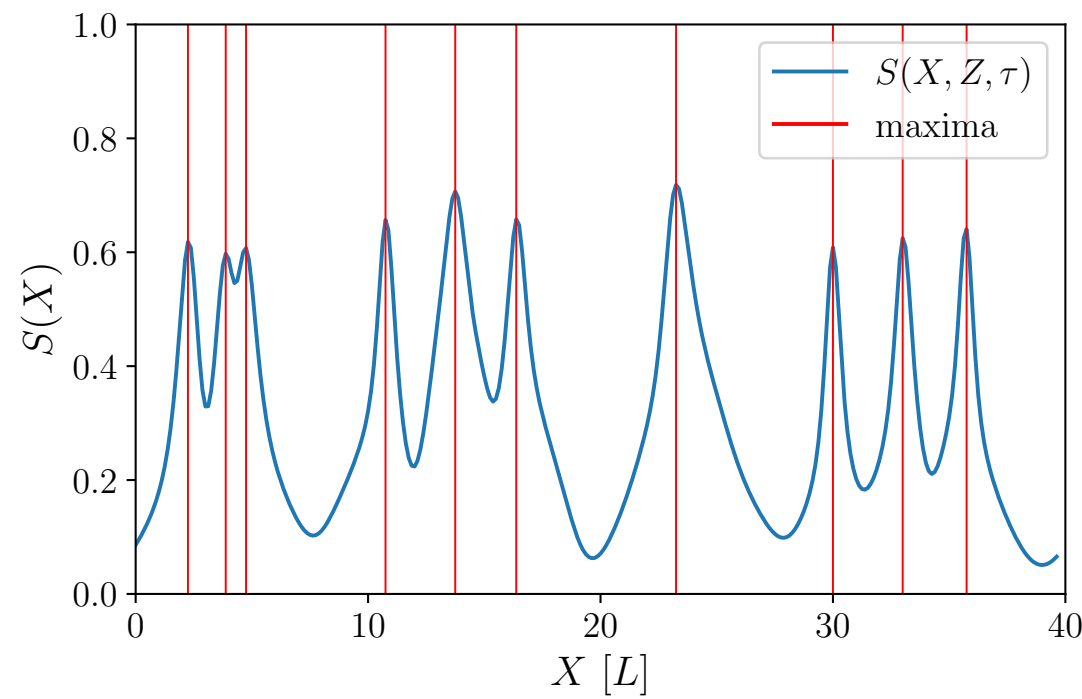

FIGURE 3.4: Detection of maxima (downwelling plumes) in the salinity profile $S(X, 1,1)$ along a line at $Z=-1$ and at time $\tau=1$ for a simulation run at $\mathrm{Ra}=100$.

I tested the same procedure for counting plumes using the velocity distribution $U_{Z}(X, Z, \tau)$ instead of the salinity, with the same results. For the sake of simplicity I resorted to always counting plumes only in the salinity field.

\section{Plume alignment}

For simulations with a non-uniform evaporation rate I am interested in the time $T_{A}$ the downwelling plumes stay in spatial alignment to the modulation imposed at the top. I determine whether the system is aligned with the top boundary at a given point in time by measuring the wavelength $\Lambda$ as described above, and comparing it 
to the wavelength of the modulation $\lambda_{\text {mod. }}$ If $\Lambda=\lambda_{\text {mod, }}$ the system is considered to be aligned. Therefore, the alignment time is

$$
T_{A}=\sum_{i=1}^{N} \Delta T_{i} \cdot \delta_{\Lambda, \lambda_{\bmod }}
$$

with the time resolution $\Delta T$ and the Kronecker-Delta $\delta_{i j}= \begin{cases}1, & \text { if } i=j, \\ 0, & \text { if } i \neq j .\end{cases}$

\section{Velocity scaling}

The dynamics in my system scale with $\mathrm{Ra}^{-\mathrm{Ra}_{c}}$. Nevertheless the speed of the dynamics I observe in my system does not scale linearly. To compare properties of systems with different $\mathrm{Ra}$, it does not suffice to compare the system states at the same dimensionless time $\tau$. To overcome this problem I define a characteristic time $\tau_{S}$ for each simulation as the time until I first observe a salinity of $S \geq 0.5$ at depth $Z=-1$. I then define the age of the system as multiples of $\tau_{S}$ (see Sec. 4.3.1 for more details).

\subsubsection{Summary}

To simulate dynamics of the porous media flows in the soil beneath the salt polygons, I solve the coupled equations for fluid and salt transport numerically in a twodimensional domain. I transform the governing equations into a stream function and vorticity representation. I solve the ensuing Poisson's equation by transforming it into Fourier space. I calculate spatial derivatives using a 6th order compact finite difference scheme and perform time integration using a 4th oder Runge-Kutta scheme with adaptive time-stepping. I choose the system width $W$ such that it encompasses at least five polygons and grid resolution such that all potentially unstable wavelengths are resolved. I performed simulations between $\mathrm{Ra}=2 \cdot 10^{1}$ and $\mathrm{Ra}$ $=2 \cdot 10^{3}$ and chose simulation durations such that the full dynamics were simulated.

Observables in the system are the number of plumes at a given time $N_{p}$ and the resulting wavelength $\Lambda=W / N_{p}$ and wavenumber $a=2 \pi / \Lambda$. I also track the plume alignment with the evaporation rate modulation at the top boundary and calculate the alignment time $T_{A}$. Last but not least I define the characteristic time $\tau_{S}$, which is the time it takes until the salinity at depth $Z=-1$ is equal or larger 0.5 for the first time. Multiples of $\tau_{S}$ are a measure of the age of the system independent of Ra.

\subsection{Experiment}

The aim of performing laboratory experiments with an artificial salt playa setup is threefold: 
(1) to explore the dynamics of the salt playa system under controlled conditions and make them observable,

(2) to explore the dynamics in very old (long running) experiments which are not accessible by simulations due to computational cost and

(3) to develop and test protocols for data collection and analysis close to field conditions.

To achieve these aims, I designed and built a Hele-Shaw setup, inspired by the common experimental approach to simulate and visualize flows in porous media by forcing the flow between two closely spaced transparent plates, as originally conceived by [126]. This setup enforces a Stokes flow with viscous drag and therefore permeability depending on the spacing of the two plates and has been used in numerous experimental investigations in $\mathrm{CO}_{2}$ sequestration $[85,75,80,84,79,82,81$, $78,76,127,83]$, the investigation of double diffusive phenomena [128, 129] as well as in investigations of systems with evaporation at the surface, which are the closest to the salt pan or salt lake circumstances $[66,60,130]$. In my approach I keep the design of the two closely spaced plates, but since I also want to test methodology for the field measurements, which involves a real porous medium i.e. sand, I fill the space between the plates with laboratory sand of know grain size. This approach is rather rare among the existing literature but has been used before by [131, 132] to investigate flows in a partially saturated porous medium.

\subsubsection{Two dimensional Hele-Shaw Setup}

The cell design is visualized in Fig. 3.5, a photograph of an ongoing experiment is shown in Fig. 3.6 (b). Two transparent polycarbonate plates with a spacing of $b=0.8 \pm 0.05 \mathrm{~cm}$ form the Hele-Shaw cell. Cell widths range from $40 \mathrm{~cm}$ to $60 \mathrm{~cm}$ and are chosen to be wide enough to provide enough lateral space for several large plumes next to each other. A large horizontal extent also minimizes the effect of the solid walls in $x$-direction on the flow observed in the middle of the cell. The cell is $20 \mathrm{~cm}$ high, allowing for observations of dynamics that are $4-150 \mathrm{~L}$ away from the top boundary in terms of natural system length $L$. A $4.8 \mathrm{wt}$.\% (or $50 \mathrm{~g} / \mathrm{l}$ ) $\mathrm{NaCl}$ solution is supplied from inlets at the bottom of the cell, which are spaced about $8 \mathrm{~cm}$ apart. The cell is completely filled with sand of which the bottom approximately $4 \mathrm{~cm}$ is very coarse sand to allow for even distribution of the water coming from the inlets. The top $16 \mathrm{~cm}$ are filled with an artificial laboratory sand of know grain size distribution. This top part is the area in which the dynamics of interest are observed.

The water inlets are connected to a reservoir holding a supply of deionized water mixed with a known salt concentration and can be opened and closed with valves. The reservoir initially is positioned such that the water table is about $2 \mathrm{~cm}$ below the top of the cell. The remaining $2 \mathrm{~cm}$ of sand are easily invaded by water which is drawn up by capillary forces. Even if the water level drops significantly over the 
lifetime of the experiment, capillary forces were always strong enough to ensure water saturation up to the top of the cell since for pore diameters of approximately $10 \mu \mathrm{m}$, a capillary rise of $1.5 \mathrm{~m}$ is expected. For very long experiment durations, reservoirs were refilled with water with the same salinity.

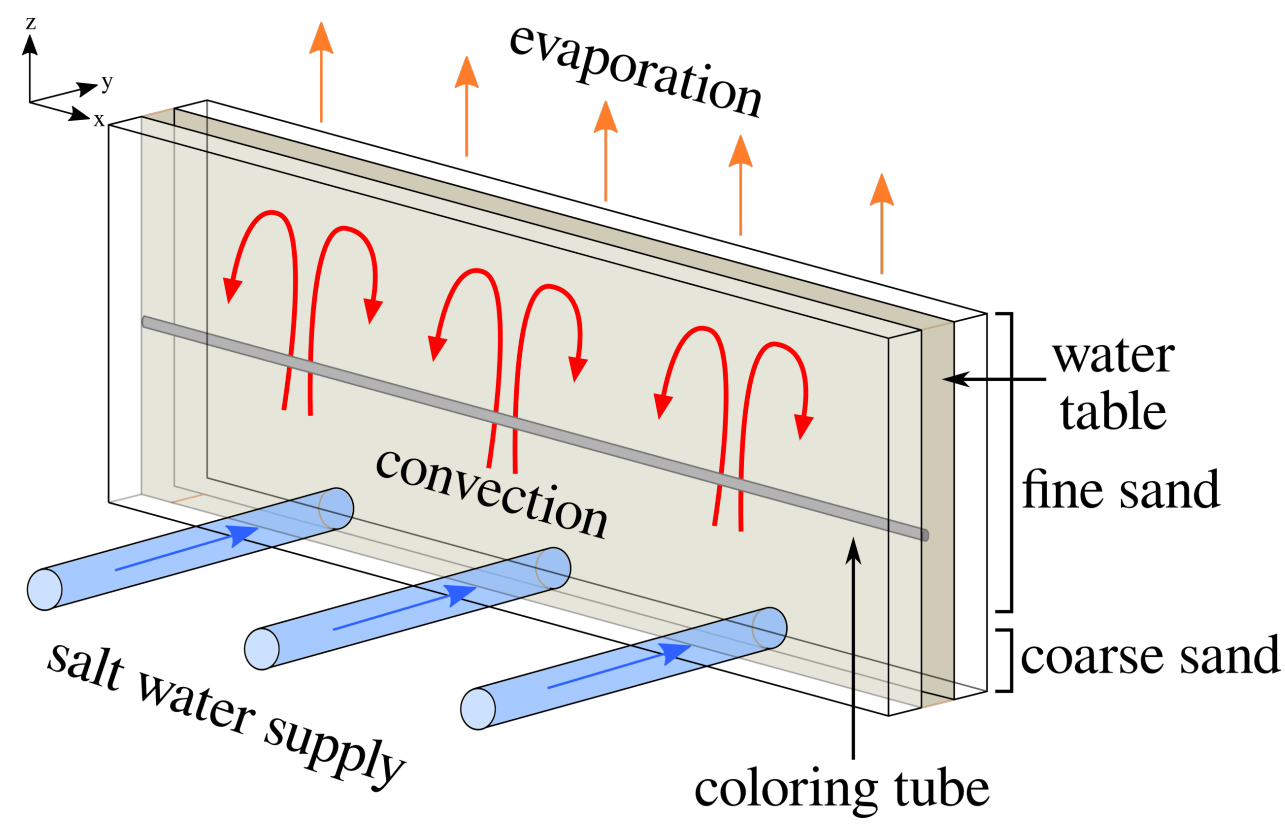

FIGURE 3.5: Sketch of the Hele-Shaw cell setup used in the experiments. Salty water is supplied from the bottom, flows through the cell and evaporates at the top, leaving the salt behind, driving convection in the cell.

In the middle of the observed area, at about $8 \mathrm{~cm}$ distance from the top of the cell, a perforated metal tube of $0.12 \mathrm{~cm}$ diameter is embedded into the sand. The tube can be used to inject a thin line of color that is deformed by the flows in the cell over time and enables me to visualize the dynamics. The tube is thin enough as to not significantly disturb the flows in the cell and far away enough from the surface as to not influence the initial instability.

Above the cell, construction lamps with a power of $400 \mathrm{~W}$ each, which emit most of their energy in the infrared spectrum, are installed to heat the cell and increase evaporation. Temperatures at the cell surface ranged from room temperature to about $60 \mathrm{C}^{\circ}$; which resembles a desert climate and is the maximum temperature the polycarbonate can endure before it starts to deform significantly. Additionally, fans are installed to increase air circulation above the cell and further increase evaporation.

The parameters that control the experiment mirror the parameters that scale the system and enter in the definition of the Rayleigh number

$$
\operatorname{Ra}:=\frac{\Delta \rho \kappa g}{E \mu \phi} .
$$

In my experiments, the gravity $g$, density difference $\Delta \rho$ and viscosity $\mu$ were always kept constant, whereas the evaporation rate $E$ was deliberately changed and sands 

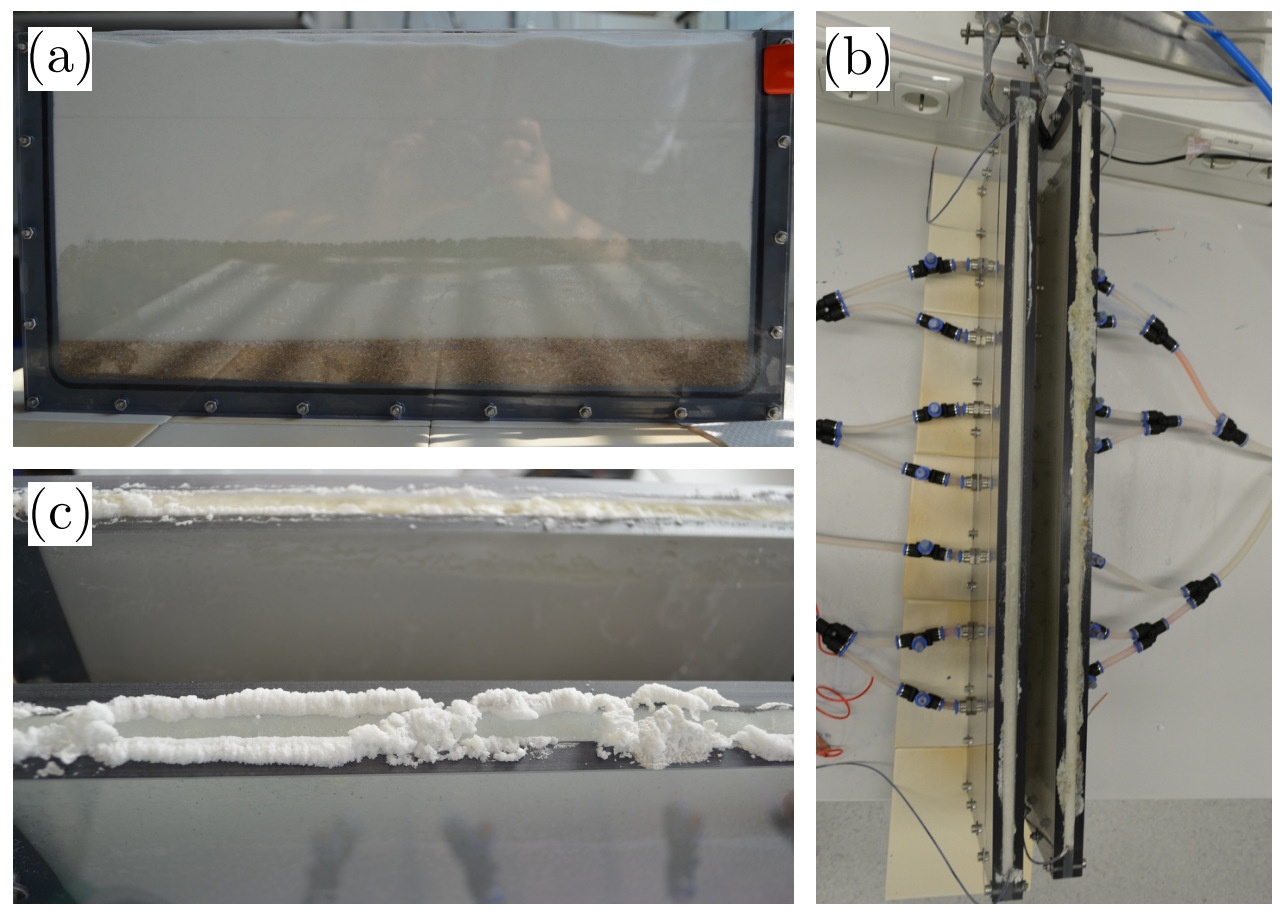

FIGURE 3.6: Experimental setups of sand-filled Hele-Shaw cells. (a) Water invasion front at setup of a cell with $40 \mathrm{~cm}$ width. (b) Installation of two cells with $60 \mathrm{~cm}$ width with water inlets. (c) Salt crust that formed at the top of the right cell.

of different grain sizes were used. This in turn influenced the porosity $\phi$ and permeability $\kappa$ of the porous matrix.

\section{Experiment Duration}

I set up the experiments in a dry state with closed inlets. Once the sand was placed in the cell, it was manually vibrated for a few seconds to anticipate sagging of the sand later on and then re-filled so the sand was level with the top of the polycarbonate plates again. Once the cell was prepared in such a way, the water inlets were opened and water slowly invaded the cell within $2-5 \mathrm{~min}$. The water invasion front was always parallel to the cell top (see Fig. 3.6 (a)), which served as a check that the even distribution of inflowing water through the coarse layer of sand at the cell bottom was working correctly. I then left the experiments to evaporate for a few days to weeks, depending on the evaporation rate. This was necessary since each experiment started with a uniform salinity distribution of $S(X, Z)=0$. The salinity gradient below the surface needed to build up first before instability could occur. Once the first patches of salt crust formed at the top of the cell, as depicted in Fig. 3.6 (c), maximum salt concentration at the top had been reached and I started to visualize the flows in the cell by injecting color into the cell. I refer to this as a "coloring experiment".

One coloring experiment lasted for 2 - 8 hours, the maximum number of coloring experiments conducted in one setup was 18 , the usual number was approximately 
five coloring experiments per setup. Setups were decommissioned after one to eight weeks.

\section{Flow Visualization}

To visualize flows in Hele-Shaw cells, both shadow-graph setups [127, 80, 133, 85] as well as color injection $[75,83,131,84,79,82,81,134,78,76,130,66,41]$ have been used in the literature in connection with Hele-Shaw setups to get accurate impressions of positions of plumes of downwelling fluid as well as their development with time. Since my setup is filled with sand, I have to resort to coloring.

To visualize flows in different areas of the cell, different techniques to apply color to the cell were used:

(a) For the first trial runs, about $8 \mathrm{ml}$ of colored solution were injected into the water inlets at the bottom and thereby plumes of upwelling fresh water were made visible as depicted in Fig. 3.7 (a).

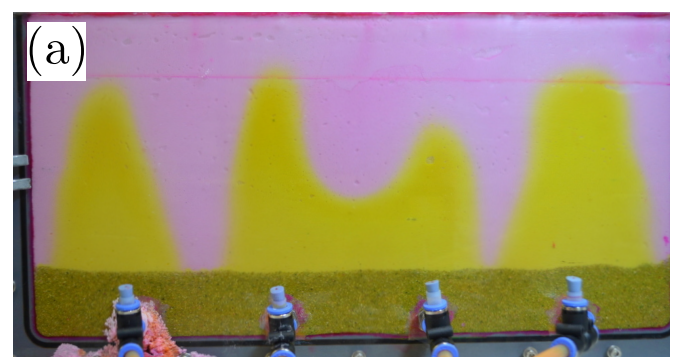

(b) To visualize flows near the surface of the cell, and hence closer to the initial instability, about $4 \mathrm{ml}$ of colored solution was applied at the top of the cell. This way plumes of downwelling salty water were made visible, as depicted in Fig. 3.7 (b).

(c) To visualize flows in the center of the cell and enable me to achieve a more accurate quantitative measurement of the spacing of the plumes, about $4 \mathrm{ml}$ of colored solution was injected via the perforated tube embedded in the middle of the cell, as depicted in Fig. 3.7 (c).

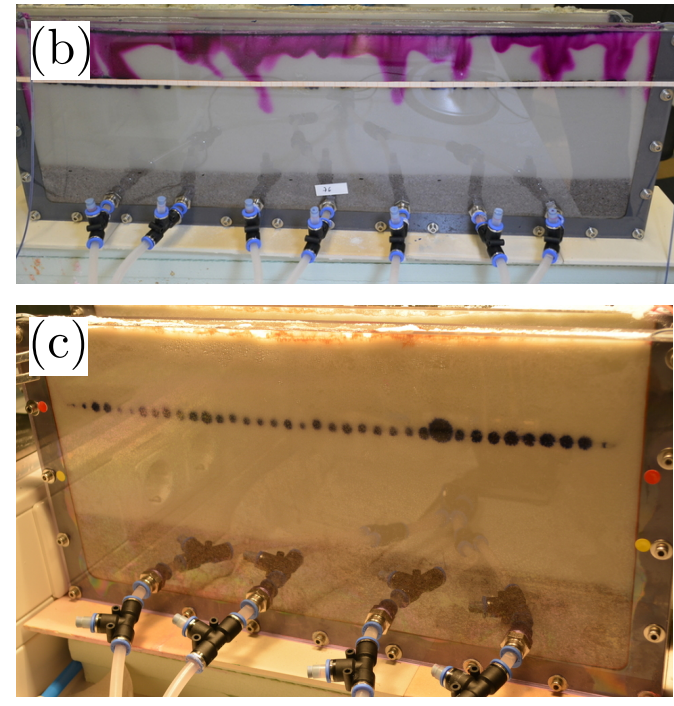

FIGURE 3.7: Visualization of flows in the Hele-Shaw cell using three different techniques: (a) coloring from the bottom inlets using fluorescein. (b) coloring from the cell top using rhodamine. (c) coloring from the coloring tube embedded in the middle of the cell using methyl blue.

To record data, a stationary camera was installed to take a digital picture of the cell in time intervals between $1 \mathrm{~min}$ and $5 \mathrm{~min}$, depending on the evaporation rate and therefore speed of the experiment. In cases (a) and (c), the density of the injected color was matched with the background density whereas in case (b) density was matched with maximum density to not artificially influence the occurring flows. 
Data recording for one coloring experiment lasted between one and six hours, depending on the evaporation rate.

To achieve the best results with regards to coloring contrast and the ability to do multiple coloring experiments within one cell, I tried different colors:

(a) Rhodamine (pink) achieved very good contrast but tended to saturate the cell with color very quickly. An example of rhodamine coloring is shown in Fig. 3.7 (b) and (a) (background).

(b) Fluorescein (yellow) achieved good contrast only if the cell had previously been colored with rhodamine. The fluorescent property of fluorescin did not take effect because the polycarbonate plates were opaque to ultraviolet light. An example of fluorescein coloring is shown in Fig. 3.7 (a).

(c) Methyl blue achieved acceptable contrast for a short while and bleached quickly after about a day. Methyl blue was used in almost all coloring experiments using the coloring tube. An example of Methyl blue coloring is shown in Fig. 3.7(c).

The number of coloring experiments that I could perform in one cell was limited by the color saturation in the cell. At some point, contrast between newly injected color and background color from previous experiments was too low to reliably perform measurements and the experiment had to be disbanded. If I changed the evaporation rate by either increasing temperature or air circulation, I did not perform coloring experiments for at least two days to give the dynamics time to adapt to the new boundary condition.

\section{Wavelength measurements}

The main observable in my experiments is the dimensional wavelength $\lambda$ of the downwelling plumes. To measure $\lambda$ in the experiment, I visualize the flows as described above. I record the time development of the color displacement via timelapse photography and measure the

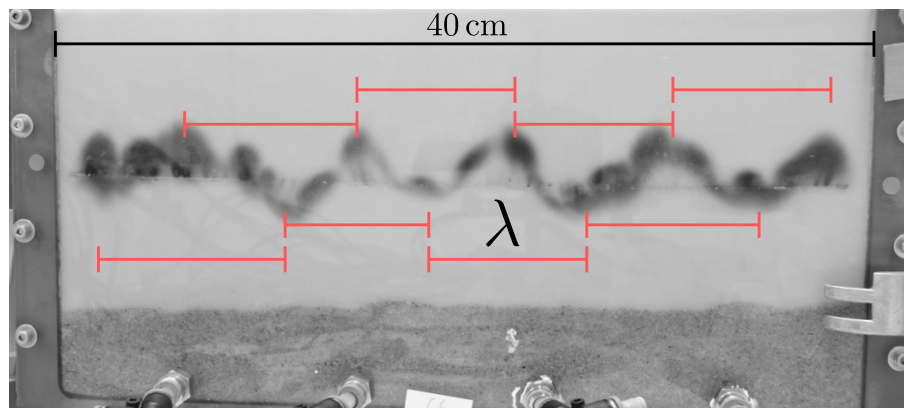

FIGURE 3.8: Displaced line of color $175 \mathrm{~min}$ after the start of the coloring experiment. Measured wavelength is indicated in red. wavelengths in the images manually using Fiji, since I can not reliably track the plumes in an automated way. This is due both to

- the fact that sometimes the coloring does not produce a completely continuous line and therefore some plumes are not traced very well by color and 
- the pronounced bleaching and therefore decreasing contrast, especially when coloring with methyl blue.

In Figure 3.8 I show an exemplary image with a line of displaced color and an indication for the measured wavelength. Wavelengths were measured as distances between the maxima and the minima of the displaced line of color respectively. Each distance is measured five times and averaged. The wavelength of the coloring experiment is then determined as average over all measured wavelengths with the standard deviation as a measure for uncertainty. I calibrate the measurements using the known dimensions of the Hele-Shaw cell. To render the wavelength dimensionless, I divide by the natural length scale $L=D / E$ of the system defined in Eq. (2.13): $\Lambda=\lambda / L$.

\section{D Setup}

Since in nature salt patterns are a three dimensional phenomenon, I also wanted to qualitatively investigate a three-dimensional experimental setup. These experiments were performed by Birte Thiede, who worked on them within the scope of her Bachelor's thesis.

The setup is a box of size $40 \times 40 \times 20 \mathrm{~cm}$ connected to a water reservoir over inlets in the bottom of the box. The box is heated from the top and air circulation is increased by fans. Pictures of the development of the salt crust are recorded by a stationary installed camera. The setup is depicted in Fig. 3.9 (a) and an exemplary image of the salt crust is depicted in Fig. 3.9 (b). Further details can be found in [135].
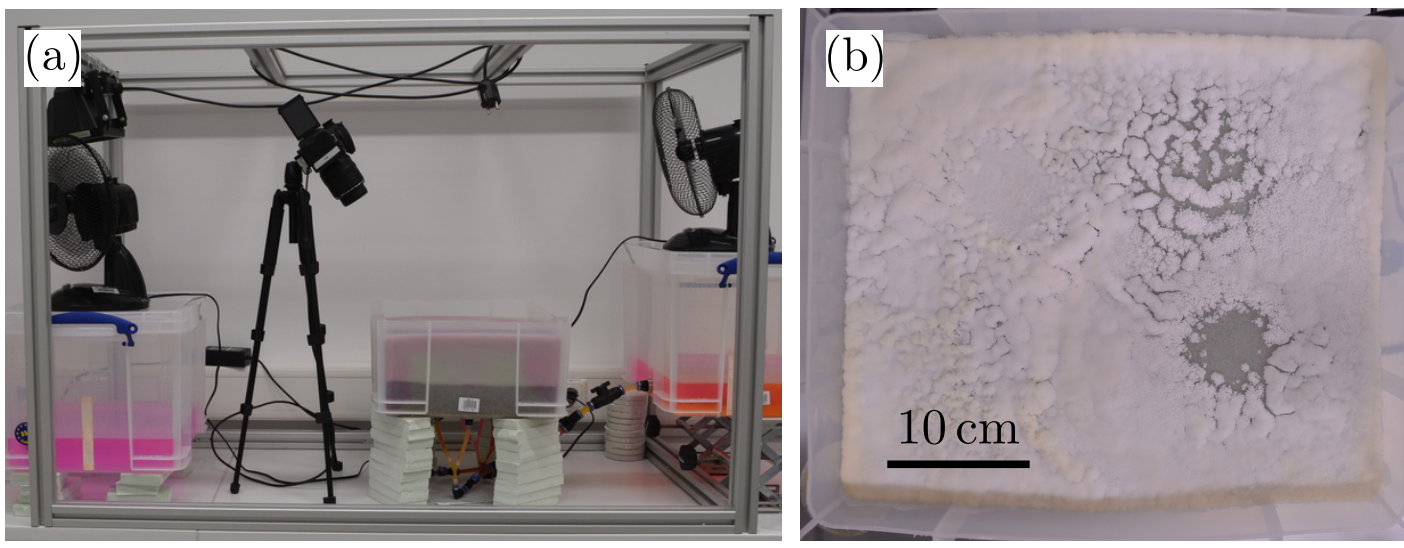

FIGURE 3.9: (a) Experimental setup for the 3D experiments with experiment in the middle, water reservoir and fan to the right and camera for time-lapse photography to the left. (b)Top-view of the crust after about two weeks of experimental duration. Images courtesy Birte Thiede.

Similar to the Hele-Shaw setup, the bottom of the box is filled with coarse sand to counteract the influence of the inlet positions on the flow. Water evaporates at the surface until a salt crust has formed. Similar to the coloring experiments in the Hele-Shaw cells, flows are also visualized by injecting density matched colored fluid 
into the box from the bottom inlets. Visualizing the flows in a three-dimensional environment is more complicated, since most of the dynamics happen inside the bulk of the sand and are not observable from the outside. To get a qualitative impression of the flows inside, three cells are dissected, removing the sand layer by layer from top to bottom. This allows for the observation of colored cross-sections of the sand in the box, which allows - to some extent - to trace the colored flows from the inlets to the surface and back.

\subsubsection{Sand characteristics}

Next to the evaporation rate, the characteristics of the sand used in the experiment change the Rayleigh number significantly. Below I will give a description of the sands I used in the experiments and it's characteristics as well as the methodology used to characterize the porous media found in the field.

\section{Grain size distribution}

Porous media are usually characterized by their grain diameter $d$. The nomenclature following the Udden-Wentworth scale [136] for the different grain sizes important for this work is given in Tab. 3.3. I will use this nomenclature later to describe the porous media I found in the field by its main and secondary component (by volume).

\begin{tabular}{c|c} 
grain diameter $d[\mu \mathrm{m}]$ & name \\
\hline $500-1000$ & coarse sand \\
$250-500$ & medium sand \\
$125-250$ & fine sand \\
$62.5-125$ & very fine sand \\
$3.9-62.5$ & silt \\
$1-3.9$ & clay
\end{tabular}

TABLE 3.3: Nomenclature of soil with different grain diameters from the Udden-Wentworth scale [136].

For the experiments, I use glass beads made of soda lime glass with a known grain diameter distribution range between $d=0-20 \mu \mathrm{m}$ and $d=400-600 \mu \mathrm{m}$ manufactured by Sigmund Lindner $\mathrm{GmbH}$. Grain size distributions for all sands are shown in Fig. 3.10.

I measure the grain size distribution of the samples collected at the field sites as well as the laboratory sand using a laser particle sizer (LPS). I use the Beckman Coulter LS 13 320, which is capable of measuring grain diameters between $40 \mathrm{~nm}$ and $2000 \mu \mathrm{m}$. Measured grain diameters are recorded in one of 93 bins. The porous medium is dissolved in water and then pumped through the laser chamber of the LPS. The LPS measures the diffraction patterns of single grains, which are then converted into grain diameters $d_{i}$ based on Mie scattering theory with a real and imaginary component of $\Re=1.556$ and $\Im=0.1$ respectively. By integration over a 


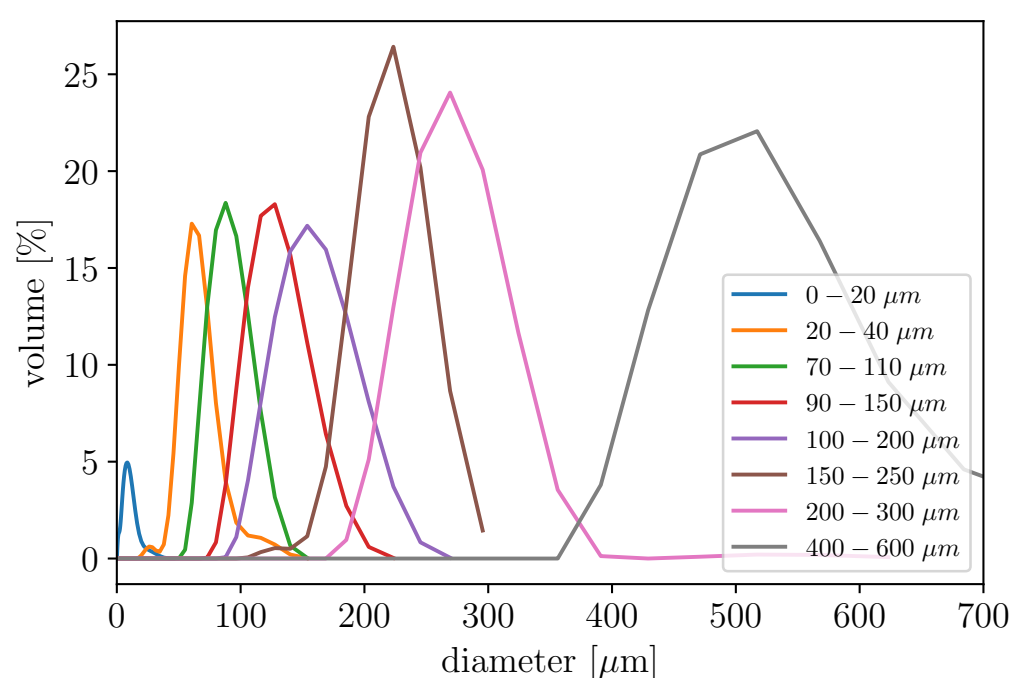

FIGURE 3.10: Grain size diameters of the artificial sand used in the experiment measured by LPS.

multitude of single grain measurements over a period of time, a volume fraction $\varphi_{i}$ of grain diameters within a certain range - or bin - of diameters is calculated. As underlying diffraction model I used quartz. Each grain size distribution measurement is an average of three independent measurements of the same sample.

\section{Sauter diameter}

Porous media in nature are very complex and hard to describe. Main contributors to this complexity is the wide distribution of grain sizes. For my model, the most important characteristic of the porous medium is the area of the surface, which is in contact with the fluid, contributes to drag and therefore affects the fluid's momentum balance. Therefore I introduce a characteristic length scale, the Sauter diameter $d_{S}[137,138]$, which is a characteristic length scale of the porous medium calculated under the constraint of constant surface area.

Given a non-spherical particle with some volume $V$ and some surface area $A, d_{S}$ is defined as the diameter of the sphere with the same surface to area ratio than the original particle. This concept can be transferred to a porous medium with a distribution of particle diameters: Given a polydisperse porous medium with total particle volume $V_{\text {total }}$ and particle surface area $A_{\text {total }}$ and some distribution of particle diameters $d_{i}$. The Sauter diameter of the medium is defined as the particle diameter $d_{S}$ of a monodisperse porous medium that gives the same ratio of total volume to total surface area:

$$
d_{S}:=6 \cdot \frac{V_{\text {total }}}{A_{\text {total }}}
$$


To calculate the $d_{S}$ from LPS data, I first need to calculate the total volume of the sample. I do this by calculating the number $n_{i}$ of particles in each bin $i$

$$
n_{i}=\varphi_{i} \cdot \frac{V_{t o t a l}}{V_{i}}
$$

This is a system of $N$ equations which is closed because $\sum_{i=1}^{N} \varphi_{i}=1$ by definition. I solve for $V_{\text {total }}$ by arbitrarily assuming that $n_{N}=1$ in the highest bin that recorded data. This is possible because I am looking for a ratio rather than an absolute value. I then calculate the $n_{i}$ for all bins and subsequently the total area given as

$$
A_{\text {total }}=\pi \sum_{i=1}^{N} n_{i} d_{i}^{2}
$$

Using the definition of the Sauter diameter from Eq. (3.14), I can then calculate $d_{S}$, which gives me a sensible estimate of the intrinsic length scale of the porous medium under the condition that drag is constant.

The main source of error for the calculation of the Sauter diameter comes from the fact that grain sizes are binned by the LPS into bins of varying size and the distribution of grain sizes within one bin is unknown. I estimate error bounds for the Sauter diameter by calculating a sample's Sauter diameter once by assuming all grain diameters are grouped on the low end of each bin and once by assuming the opposite.

\section{Permeability}

While Kozeny and Carman $[139,140]$ made a first approximation which relates the permeability of a porous medium to its porosity $\phi$

$$
\kappa=d^{2} \frac{\phi^{3}}{180(1-\phi)^{2}} .
$$

This relation, known as the Carman-Kozeny approximation, is not very accurate for packs of aspherical and polydisperse particles [141]. To improve in this approximation, numerical simulations were conducted by [141] to find the dimensionless permeability for different types of porous media, both with monodisperse and spherical as well as polydisperse and aspherical beads. Following their guidance, I can define the dimensionless permeability $\kappa^{\prime}$ as

$$
\kappa^{\prime}=\frac{\kappa}{d_{S}^{2}}
$$

where $d_{S}$ is the Sauter diameter of the porous medium, as defined in Eq. (3.14). Following $[142,143], \kappa^{\prime}$ can be expressed as

$$
\kappa^{\prime}=\gamma(\phi) \kappa_{0}\left(x_{1}, x_{2}, \ldots\right)
$$


where $\gamma(\phi)$ denotes the dependency of the dimensionless permeability on the porosity and $\kappa_{0}\left(x_{i}\right)$ is a geometric parameter that depends on the specifics of the porous medium such as grain shape, the tortuosity of the medium and connectivity. The best fit for aspherical particles with an aspect ratio of $\alpha=1.28-2.18$ is

$$
\kappa^{\prime}=0.11 \phi^{5.6}
$$

which is also in agreement with the empirical fit to experimental data by [144].

Since the permeability scales as $\kappa \propto d_{S}^{2}$ (see Eq. (3.15)), it is the most convenient parameter to change the Rayleigh number of the system within a very large range, by changing the grain diameter of the porous medium used in the experiments.

Since the uncertainty resulting in an approximation of the permeability of the system has the largest influence on the uncertainty of the Rayleigh number, I measured permeabilities for most grain sizes used in the experiments experimentally. This was possible because I had access to enough artificial sand to perform the permeability measurements and permeabilities were high enough to perform the measurements within a reasonable amount of time. I was not able to conduct similar measurements for the samples of porous medium collected in the field.

The permeability measurements were performed by Birte Thiede. Permeabilities for the grain sizes used in the experiments are listed in Tab. 3.4.

\begin{tabular}{c|c|c}
$d[\mu \mathrm{m}]$ & $\kappa\left[\mathrm{m}^{2}\right]$ & $\phi \pm 10^{-2}$ \\
\hline $70-110$ & $0.77 \pm 0.13 \cdot 10^{-11}$ & 0.32 \\
$90-150$ & $1.44 \pm 0.1 \cdot 10^{-11}$ & 0.30 \\
$100-200$ & $1.67 \pm 0.12 \cdot 10^{-11}$ & 0.28 \\
$150-250$ & $4.7 \pm 0.2 \cdot 10^{-11}$ & 0.24 \\
$200-300$ & $8.27 \pm 0.3 \cdot 10^{-11}$ & 0.21
\end{tabular}

TABLE 3.4: Permeabilities measured for the sand beads used in the Hele-Shaw cell experiments measured by [135].

\section{Porosity}

It is beyond the scope of this work to accurately model the porosity of the various mixtures of sand, clay and silt found at the field sites. I use the Sauter diameter as a characteristic length scale of the porous medium and an indicator of clay content: the smaller $d_{S}$, the higher the clay content of the porous medium. A more accurate assessment of the clay content is possible via the grain size distribution.

Porosity increases, if the soil contains significant amounts of clay. The porosity has a minimum at a clay content of about $25 \%$ [145] and then increases again for larger grain diameters. Since $d_{S}$ for all the field sites was rather small and clay content significant, I assume that porosity will linearly increase with decreasing $d_{S}$. I use the porosities measured experimentally by [146] for wet packed polydisperse porous media of different grain sizes and apply a linear fit to the empirical data, shown in Fig. 3.11 to calculate porosities for my samples. 


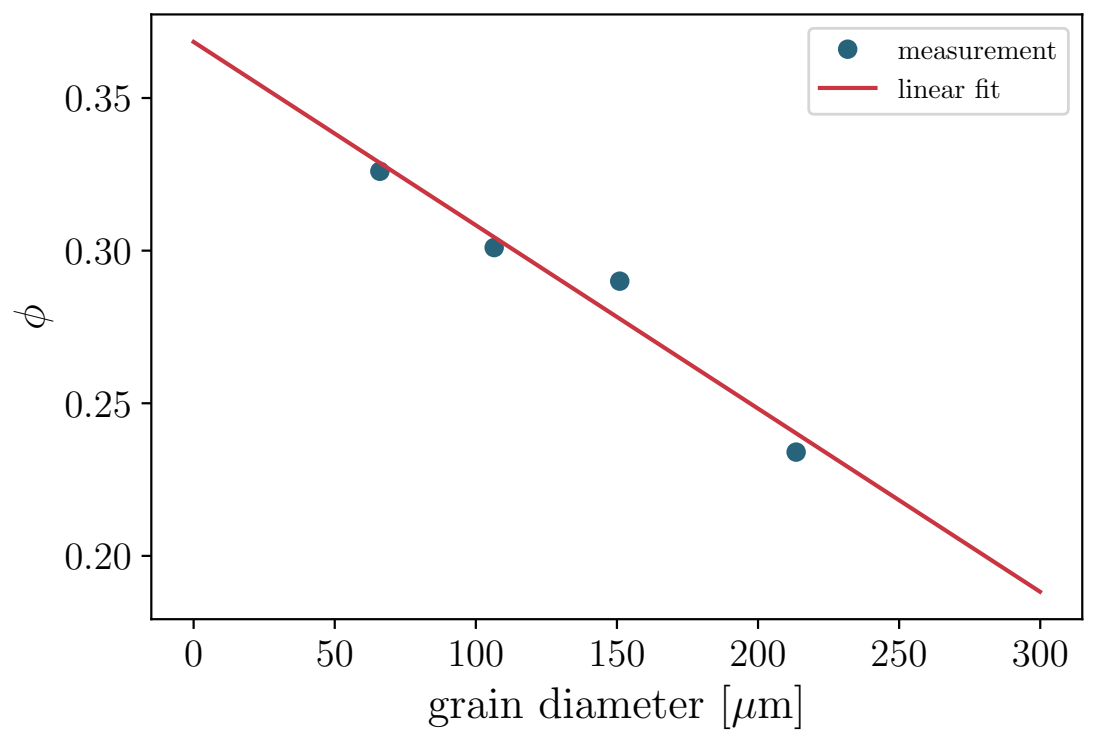

FIGURE 3.11: Linear fit to the empirical relationship of porosity to grain size for dry and wet packs of sand determined by [146].

For the fit I correlate the porosity measured by [146] with the middle of the grain size range of each measurement. To calculate the porosity for a specific sample, I use the Sauter diameter of that sample and calculate the porosity as

$$
\phi\left(d_{S}\right)=0.37-6 \cdot 10^{-4} d_{S} \frac{1}{\mathrm{~m}} .
$$

For the laboratory sand, the manufacturer only provides data about the porosity of the dry packed sand, which is considerably higher than the porosity of a wet pack [146]. Therefore, to determine the porosity of the sand used in the experiments, I use the same methodology as described above. Porosities calculated this way are listed in Tab. 3.4.

\subsubsection{Evaporation Rate}

\section{Controlling evaporation rate}

The evaporation rate $E$ was the main parameter that was varied during the experiments. Increasing the evaporation rate drives the dynamics at a higher speed and allows the observation of the dynamics on a timescale of hours in the laboratory instead of the weeks and months they take in the field. Moreover, the evaporation rate is expected to scale the system via the natural length scale $L=D / E$.

Evaporation rates were smallest at room temperature without additional air circulation above the setup and reached values of approximately $3 \cdot 10^{-8} \mathrm{~m} / \mathrm{s}$. It has to be noted that I was not able to completely and reliably control evaporation rates, since evaporation also depends on air humidity. Since I conducted the experiments 
in a laboratory which was temperature but not humidity controlled, relative air humidity varied between about $20 \%$ on dry days and $55 \%$ on humid days. I measured humidity in the laboratory and found it to change on timescales of days to weeks, mostly depending on the outside weather conditions. Since usual coloring experiment durations ranged between $1-6$ hours, I assumed that evaporation rate changes due to changes in air humidity occur slow enough to not significantly alter the dynamics inside the cell.

By heating the cell surface to about $60^{\circ} \mathrm{C}$ and placing several fans next to the cell, I achieved maximum evaporation rates of $E \approx 1.2 \cdot 10^{-6} \mathrm{~m} / \mathrm{s}$ or $10 \mathrm{~cm} /$ day, which is about 250 times faster than evaporation rates in the field setting. It has to be noted that by heating from the top, a temperature gradient is induced in the fluid, which also results in a density gradient. The difference between the density of water at room temperature and water at $60 \mathrm{C}^{\circ}$ is about $1.5 \%$ [109]. This gradient

a) acts in the opposite direction than the density gradient induced by differences in salinity and therefore has a stabilizing effect on the system, and

b) is about one order of magnitude smaller than the density gradient induced by the salinity gradient.

This is why for the rest of this work I choose to neglect the density gradient caused by a potential temperature gradient in the system.

\section{Measuring evaporation rate}

Since I expected the evaporation rate to directly control the scaling of the dynamical system, measuring it as accurately as possible was of high importance. The easiest way to measure evaporation rates at the cell surface is to track changes in the water level of the reservoir feeding the cell. This was possible because every reservoir was connected to only a single cell and the reservoir-cell setup was a closed system with the only opening allowing for fluid loss being the surface of the cell. While influencing evaporation rates by heating or air circulation was straightforward, measuring evaporation rate was not trivial. Problems I encountered were

- Very slow changes in water levels of the reservoirs between $0.1-0.5 \mathrm{~mm} /$ day.

- Very long lifetime of the experiments between one week and several months and long duration of single coloring experiments of several hours.

- Menisci and bubbles in the system that made visual determination of the waterlevel difficult.

- Occasional leakage of the experimental setup.

First approaches with capacitor based sensors to track water levels in the reservoirs failed, because the capacitance change caused by minute water level changes were 

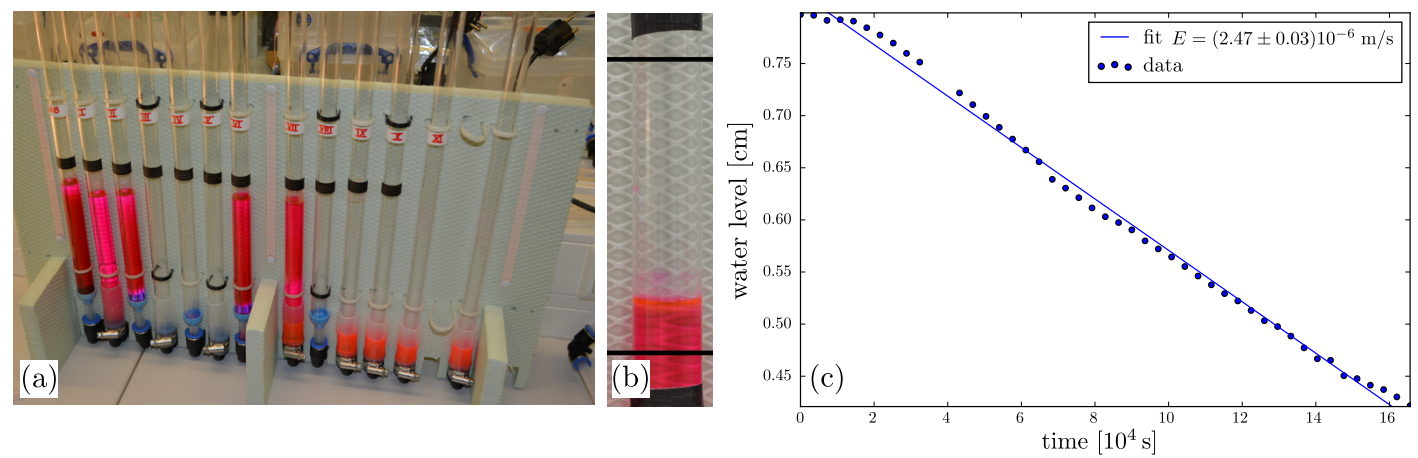

FIGURE 3.12: (a) Evaporation rates were measured by tracking the water levels in the reservoirs using manometers.(b) Single manometer with black marker strip at the top and colored water in pink. (c) Exemplary fit to a measurement of water level in the reservoir.

drowned out by the capacitance change caused by temperature variations in the laboratory. The laboratory was temperature controlled within $2-3^{\circ} \mathrm{C}$ but the diurnal temperature changes within this small range were enough to disturb my measurements in a way I was not able to counteract by signal processing. Attempts to track water levels by ultrasound or infrared based distance measurements also proved to not by reliable enough because sensors tended to fail repeatedly due to the very humid climate conditions inside the reservoirs and the very long measurement durations.

The second approach was to track water loss in the reservoir by measuring weight loss. This is, in principle possible, by using high accuracy balances able to track weight changes on the order of micrograms. Nevertheless, the readings of these balances tended to slowly drift over the course of several hours. If digital drift-control was enabled, the balances did not pick up on the signal caused by the very slow weight loss and interpreted it as part of the drift. Moreover these balances were also susceptible to small changes in atmospheric pressure caused by weather changes. Therefore I did not trust the measurements acquired via this method.

Leakage of the experimental setups occurred in approximately one in four setups. Leakage was caused by corrosion of metal parts of the inlet tubes due to exposure to salt water for extended periods of time or the polycarbonate plates becoming brittle over time. Experiments were continuously checked for leakage and obviously leaking experiments were excluded from any quantitative measurements, yet sometimes used for qualitative assessments of flow patterns.

The last and successful approach was to track water levels visually with installed cameras. For this purpose, I connected each reservoir to a very tall and thin stationary manometer (to prevent significant evaporative loss through the manometer). Water in the manometer was colored with Rhodamine to increase contrast and a picture of the water level in the manometer was taken every hour. This setup is depicted in Fig. 3.12 (a). The images were then post-processed digitally using the following image processing protocol for every image: 
- Divide the image into areas containing (1) water, (2) empty manometer tube and (3) black marker strip at the top of the image. This segmentation is indicated in Fig. 3.12 (b) by the horizontal black lines.

- Take only the red channel of the images, since it provides the best contrast when using red dye (rhodamine) in the manometer.

- Segment each sub-image by

- blurring the image with a small $(5 \times 5$ pixel) kernel size to remove high frequency noise stemming from thermal fluctuations of the CCD chip of the camera,

- cutting the image into three separate images containing the areas corresponding to (1), (2) and (3),

- applying a thresholding operation to each sub-image to segment it into foreground and background using the threshold $T=10+\frac{1}{N} \sum_{i}^{N} I_{i}$ with $I_{i}$ the intensity value of each pixel and

- removing disconnected small objects and filling in small holes, leaving only objects with a size $\geq 10^{4}$ pixels in the image.

- Calculate the distance between the lower edge of the marker strip and the upper edge of the water in the tube at at least 5 different positions and average over the distances to calculate the overall water level position.

- Conversion from pixels to centimeters was done using repeated measurements of a known distance on a millimeter paper placed next to the manometer as calibration length.

A linear fit to the water level positions over time then yields a very accurate measurement of the rate of water loss from the reservoir, as depicted for an exemplary measurement in Fig. 3.12 (c). I calculated evaporation from the cell by multiplying the rate of change of the water level by a conversion factor translating the surface area of the reservoir to the surface area of the cell and the porosity of the sand, since water can only evaporate from areas of the porous medium filled with fluid.

\subsubsection{Salt Concentration}

\section{Controlling salt concentration in the experiment}

Since the parameter of importance for my system is the density of the liquid, which is dependent on the sum of dissolved salts, for my experiments I choose to simplify the system and add only one salt species, whereas in nature several dissolved salt species are present. Nevertheless, there is a range of studies that specifically investigate the role of different chemicals and chemical reactions in the diffusion process both experimentally $[147,133]$ as well as in the field setting $[148,27,26,149,24]$. 
I added $\mathrm{NaCl}$ purchased from Sigma Aldrich with a purity of $\geq 99.5 \%$ to the deionized water supplied at the bottom of the Hele-Shaw cells. The salt was added to the water, mixed until completely dissolved and then placed in the reservoir. I choose to keep background salt concentration fixed at $50 \mathrm{~g} \mathrm{NaCl} / 1$. Since the maximum density is $\rho_{S}=1.200 \mathrm{~g} / \mathrm{cm}^{3}$ for $\mathrm{NaCl}$ at $21^{\circ} \mathrm{C}$ and atmospheric pressure [109] and the background density is $\rho_{b}(50 \mathrm{~g} / \mathrm{l})=1.038 \mathrm{~g} / \mathrm{cm}^{3}$, the density difference is $\Delta \rho=0.162 \mathrm{~g} / \mathrm{cm}^{3}$.

\section{Measuring salt concentration in the experiment and the field}

If the emergence of salt polygons is driven by convection below the patterns, horizontal as well as vertical concentration gradients should be detectable in the pore water of the sand underneath the patterns. To measure these gradients, I needed a reliable method to measure salinity in a porous medium in the field. I tested and refined the measurement protocol in my Hele-Shaw cell experiments to later use it on samples collected in the field and to confirm the presence of such gradients in the analogue system.

Standard salinity measurements rely on the change of conductivity of the fluid with increased number of ions dissolved in it. Nevertheless in a porous medium these measurements can be rather unreliable, especially when the medium is not completely saturated with water (anymore). Therefore I resorted to gravimetric measurements as my main method of determining salt concentration. I collected samples of about $1 \mathrm{~cm}^{3}$ of porous medium containing the salty water both from my experiments as well as from the field sites, which were subsequently analyzed in the laboratory.

Samples were transported in sealed containers to a laboratory equipped with a high precision Denver Instrument SI-234 balance with a precision of $\pm 0.1 \mathrm{mg}$ ans well as an oven to dry the samples. Gravimetric analysis of salt concentrations was conducted in the following steps:

1) Careful extraction of the samples from the containers into a crystallization dish and measurement of the initial mass of the mixture of sand, salt and water.

2) Drying of the sample in an oven at $80^{\circ} \mathrm{C}$ until all moisture had visibly vanished or for at least 24 hours, followed by weighing to measure the amount of moisture that vanished from the sample by difference to the sample mass before drying. Care was taken to let the samples cool down completely before weighing, because of the temperature sensitivity of the balance.

3) Dilution of the sample with roughly $50 \mathrm{ml}$ of deionized water followed by sedimentation of the solid sample components for roughly 24 hours and careful extraction of the liquid part, containing the dissolved salt using a syringe. This step was repeated two times, and the extracted liquid was placed in a separate crystallization dish. 
4) Drying of both the solid and liquid part of the sample in an oven at $80^{\circ} \mathrm{C}$ until all moisture had visibly vanished or at least 24 hours had passed. For the liquid part, drying sometimes took considerably longer (on the order of a week). Subsequent weighing of both the solid and the liquid part.

The comparison between the weight of the dry sample composed of salt and sand with the weight of the dry sample without the salt gives an indirect measure of the salt content in the sample whereas the weight of the salt crystallized in the dish gives a direct measure of the salt content. The difference between both weights gives an indication of the reliability of the analysis. If both weights are within the accuracy of the balance, the sample mass was conserved and no mistakes were made during the measurements.

For analysis of the samples from experiments as well as from the first field campaign, I complemented concentration measurements by conductivity measurements. I measured conductivity after step (3) of the protocol described above. Conductivity was measured using a Eutech Instruments PC 2700 conductivity meter equipped with a 2-electrode epoxy/platinum cell with a nominal cell constant of $1.0 \mathrm{~cm}^{-1}$. The cell was calibrated to the measurement range of $10^{3} \mathrm{mS}$ to $10^{5} \mathrm{mS}$, depending on the total dissolved salts in the liquid samples. Calibration was done before each measurement session, using stock solution with known conductivity at a given temperature. Cell constants after calibration ranged between 0.145 and 0.155 . The linear temperature coefficient was set to zero to prevent automatic temperature correction by the measurement device. Temperatures in the laboratory ranged between $21^{\circ} \mathrm{C}$ and $23^{\circ} \mathrm{C}$ and were recorded for each individual measurement. Temperature correction of the conductivity readings was then done manually. Conductivity was observed to fluctuate by about $5 \%$ between readings and readings needed up to $30 \mathrm{sec}$ to stabilize, therefore for each measurement three readings were performed and then averaged to arrive at the final conductivity. Between each reading the sensor was cleaned with deionized water.

I concluded that the readings from both the gravimetric as well as the conductivity measurement approaches are very similar, with the gravimetric measurements being more reliable and more time efficient. Therefore I will only refer to results from gravimetric measurements in the remainder of this work.

Since the measurement protocol for salt concentration developed in the experiments was also used in the field, reliability of an individual measurement was an important factor: for the two field campaigns a total of about 3000 samples were processed in a highly streamlined manner, and mistakes in the analysis of a single sample could not be completely excluded. Especially for the samples collected in the field, indirect measurements of salt content turned out to be slightly more reliable than direct measurements. This was mainly due to the fact that the salt in the crystallization dish sometimes did not dry completely and it was very hard to determine whether all water had really evaporated. This was due to some soluble biological components (see Sec. 3.3.3 for details) that were extracted together with 
the dissolved salt and tended to form a thin film or tiny pockets enclosing the liquid which hindered evaporation.

In the following I therefore use the indirect measurement of the mass of the soluble components divided by the measurement of the mass of the water contained in the sample as a measure for salt concentration in the sample.

\subsubsection{Summary}

To study an analogue system of advection-diffusion dynamics in experiments, I implemented a quasi two-dimensional Hele-Shaw setup. The Hele-Shaw cells are filled with sand with known characteristics and supplied with saline water with a known salt concentration from the top. At the top, water evaporates with a known rate. Flows in the cells are visualized by injection of color. This experimental setup allows me to both investigate the onset of convection and accurately measure the wavelength of the ensuing convective dynamics at a known Rayleigh number. Additionally, the experiments allow me to develop and test measurement protocols for the field studies. My Bachelor student Birte Thiede also implemented proof-of-principle three dimensional experiments, which I mention briefly.

\subsection{Field Research}

As the aim of this work is the study and explanation of the phenomenon of salt polygon formation in nature, a central part is the acquisition of data from regions in the world where salt polygons can be observed. This is especially important since research concerning geophysical processes that take place in the underground often relies heavily on indirect evidence [68]. Direct hydrological or geological evidence in the form of samples taken from the underground, which allow for direct measurement of the observables is rare. This is a shortcoming that I wanted to address with the collection and analysis of direct evidence from the field.

The US was the most suitable lo-

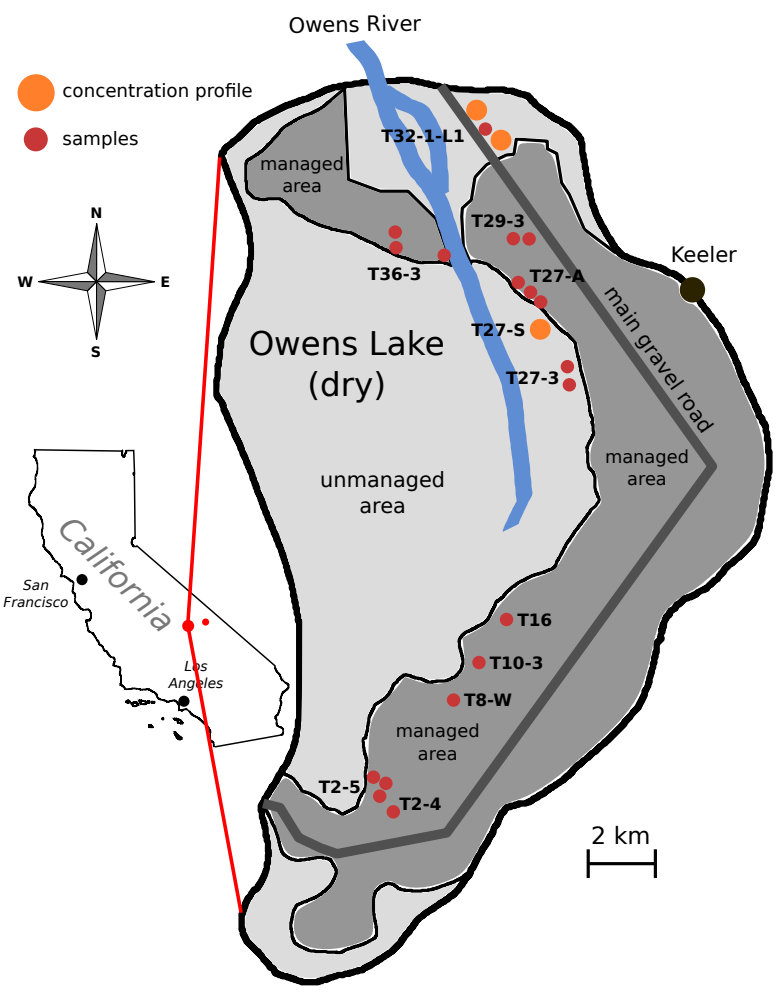

FIGURE 3.13: Schematic map of Owens Lake in central California, USA. cation for my field research, since other regions such as Chile, Tunisia or Iran would have been more difficult to reach because of political instability or remoteness. 
I carried out two field campaigns in central California in November and December 2016 and January 2018. For both field campaigns I was accompanied by my supervisor Lucas Goehring, who helped with the sampling and Joanna Nield, who conducted the terrestrial laser scans (for details see Sec. 3.3.2). During the first campaign I conducted a broad survey of several sites in the region. I focused on Owens Lake and Badwater Basin but also visited Soda Dry Lake and Bristol Playa, where I found no polygons or polygons significantly disturbed by salt mining operations. Additionally I established sampling and measurement protocols. During the second field campaign I returned to Owens Lake and focused on the collection of samples to compile high resolution subsurface salt concentration profiles.

Sites I visited at Owens Lake are indicated as colored dots in the schematic map of the dry lake shown in Fig. 3.13. Measurement sites at Badwater Basin are indicated as dots in a schematic map of the salt flats shown in Fig. 3.14. The schematic map corresponds to the part of the salt flats south of the main tourist pathway.

Salt concentration profiles along cross-sections below polygons, which show horizontal salt concentration variations aligned with the patterns visible on the surface, are the most direct proof of my hypothesis. While I tried to compile reliable profiles for all sites I visited, I was not successful in doing so at most of the sites. Especially the profiles compiled during the first field campaign did not have a high enough resolution and precision to test my hypothesis of the existence of horizontal concentration gradients in the underground below the patterns. I discuss the problems I encountered with measuring concentration profiles in more detail in Sec. 4.4.2.

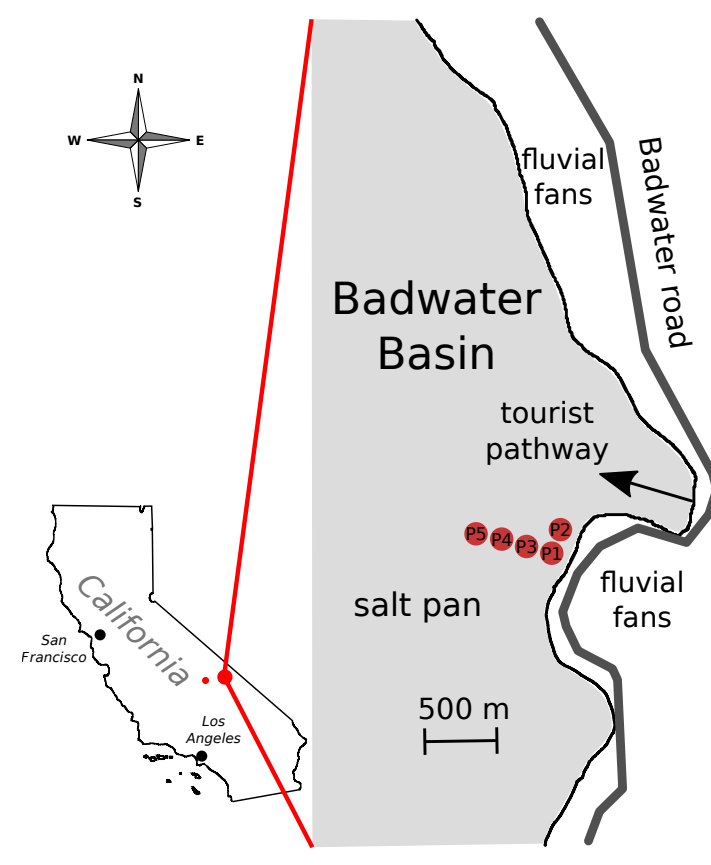

FIGURE 3.14: Schematic map of Badwater Basin in the Death Valley, central California, USA. Sampling sites are indicated as red dots.

Orange dots in the schematic maps correspond to sites where I collected full subsurface concentration profiles with high enough resolution to resolve horizontal concentration gradients. Red dots correspond to sites were I measurement only the Rayleigh number and pattern wavelength from the collected data. GPS positions of all field sites is listed in the Appendix, Sec. A.1. Descriptive information about each site is provided in the Appendix, Sec. A.2 to A.28.

In the following, I will describe the protocols, tools and sources used to measure or approximate the different observables in the field, which in turn are used to confirm the different predictions I made in Sec. 1.4. Observables are 
- Subsurface salt concentration profiles (sampling, gravimetric analysis)

- Pattern length scales (terrestrial laser scanner)

- Permeability (sampling, laser particle sizer analysis)

- Evaporation rate (literature)

- Chemical composition of salts (sampling, X-ray diffraction analysis)

- Temperature and humidity (direct measurement, public weather records)

- General description of the area (images, information from local researchers, public weather records)

\subsubsection{Subsurface sampling}

The advection-diffusion model described in Sec. 1.3 predicts the emergence of convection cells and therefore horizontal and vertical salt gradients in the underground. These gradients should be measurable and co-located with the pattern visible at the surface. By measuring the salt gradients directly, I connect the subsurface dynamics with the surface patterns.

Obtaining information about the salt concentration in the soil below the surface is not straight forward. Consideration has to be given to the fact that the naturally established dynamics in the ground will be disturbed by sampling. Therefore, at first glance, indirect measurement of salt concentration seems appealing.

Indirect measurement methods like electrical resistance tomography (ERT) have been used successfully to detect differences in ion concentration in the underground. ERT yields sufficiently accurate results on small length scales on the range of centimeters only under very controlled circumstances in the laboratory [150,151]. For field scenarios, only very large length scales with a resolution on the order of several meters can be resolved so far $[88,152]$ with the precision required for my purposes. It has to be mentioned that recent advances in ERT usage in the field [153] approach the length scales of salt polygons and could be considered for future studies. Given the fact that I did not have access to expertise with the use of ERT, I decided to take samples to analyze salt concentration gravimetrically.

Circumstances in the field can be very noisy. The subsurface can contain unexpected features and disturbances, such as buried metal parts, pebbles or submerged secondary salt crusts. Therefore collecting direct hydrological evidence in the form of samples, which allow for visual inspection, is of great importance to assess the reliability of each sample. Bringing samples back from the field also has the added benefit that measurements such as the determination of grain sizes, the proportion of biological material and a characterization of the minerals are possible.

I collected samples from cross-sections below salt polygons employing two different methodologies: 
1) Digging a trench and then collecting samples from one trench wall, as shown in Fig. 3.15 (a).

2) Drawing cores and then collecting samples from the cores for the wetter sites as shown in Fig. 3.15 (b).

For both sampling methodologies, I collected samples from directly below the crust down to a depth of about $100 \mathrm{~cm}$. During my first field campaign, I sampled with a vertical resolution of about $20 \mathrm{~cm}$ and a horizontal resolution of $30-45 \mathrm{~cm}$. I sampled 17 polygon cross-sections and collected about 500 soil samples in total which were returned to the lab for further processing. During my second field campaign, I increased the resolution to $10 \mathrm{~cm}$ and $15 \mathrm{~cm}$ in the vertical and horizontal directions respectively, increasing the number of samples per polygon by a factor of four. During my second field campaign I sampled 10 individual polygon cross sections and one line crossing several polygons. Additionally, I sampled several cross-sections for one single polygon, to create a 3D representation of the subsurface salinity. During the second field campaign I collected about 3000 soil samples.

Each sample contained sand permeated by salty water. Samples were placed in watertight containers directly after collection and sealed with parafilm. This was important to prevent the loss of humidity between sample collection and salt concentration measurement in the laboratory. As control of these losses, I tracked the weight of at least one sealed container per sampled polygon over months by measuring the container weight approximately every three months. Humidity loss was minimal with approximately $10^{-4} \mathrm{wt} . \%$ over the course of 6 months.

\section{Coring}

Most of the sites had a water table at $5-10 \mathrm{~cm}$ below the surface, with liquid reaching up to right below the salt crust. This made it impossible to dig trenches. I sampled these sites by drawing soil cores along a line spanning the polygon ridge to ridge. I used a Dutch Gauge Corer with a diameter of $3 \mathrm{~cm}$. After drawing the core, the corer was quickly put in a horizontal position to prevent brine from running down the corer and contaminating the sand farther down. The surface of the core was then scraped away to further minimize contamination and individual scoops of sand with a volume of about $3-10 \mathrm{~cm}^{3}$ were collected with a spatula. The spatula as well as the corer were cleaned with deionized water and dried before each use. Coring was significantly quicker than trenching and allowed me to sample up to 5 polygons per day.

\section{Trenching}

Drier sites with a lower water table at a depth of $70-100 \mathrm{~cm}$ allowed for the digging of trenches. I preferred this method over coring, since it avoided potential contamination of samples by freely flowing brine or pieces of salt pushed downwards 


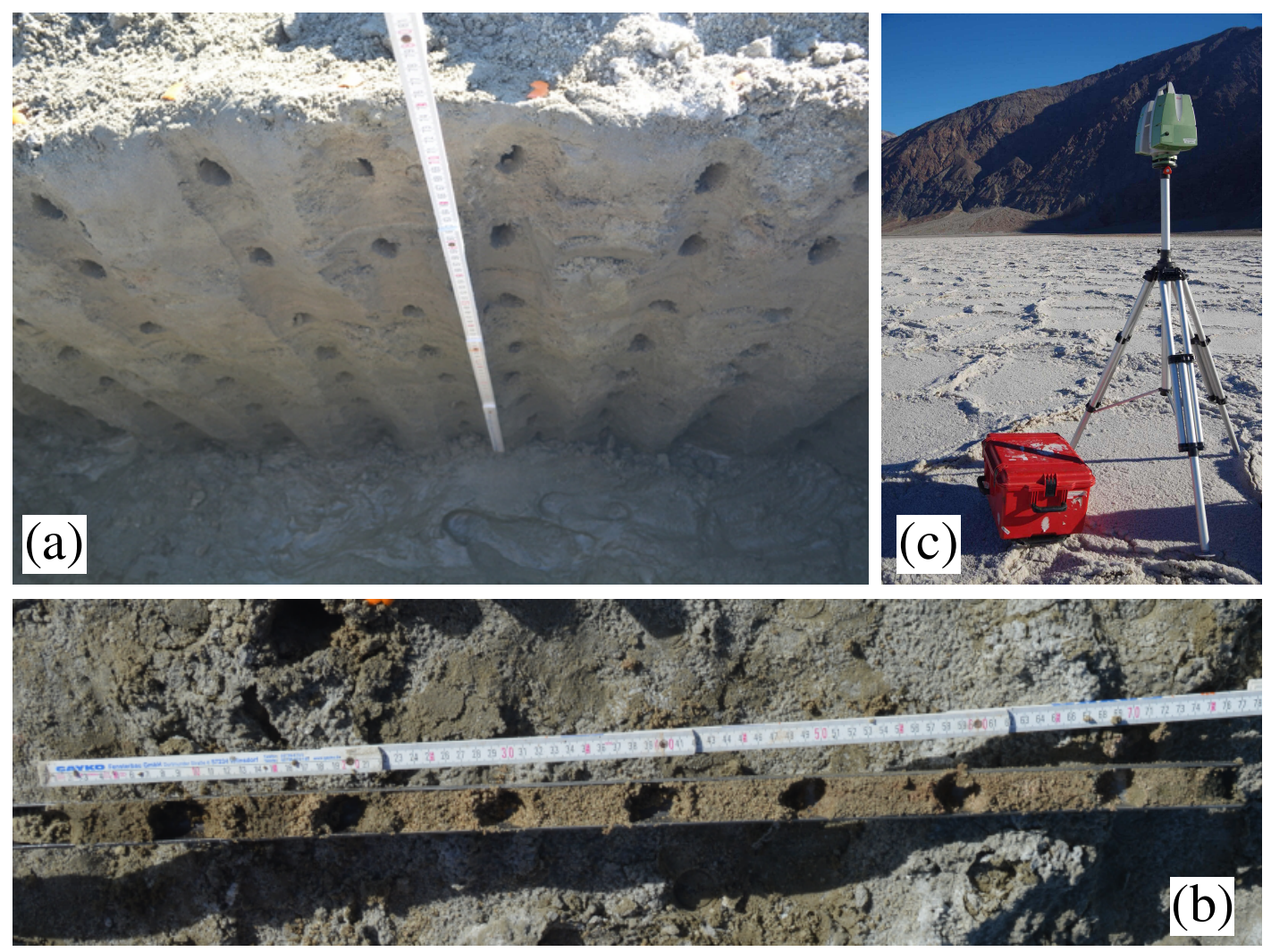

FIGURE 3.15: Sampling by (a) digging a trench and then collecting samples from one trench wall and (c) drawing a soil core and then collecting samples from the core. (b) image of the terrestrial laser scanner (TLS) used to record the surface relief and measure the pattern wavelength.

by the corer. Nevertheless, digging trenches was also significantly more time consuming than coring. We were able to dig two trenches per day. The trenches were about $100 \mathrm{~cm}$ deep, $40 \mathrm{~cm}$ wide and spanned the polygon ridge-to-ridge, extending 20-30 cm further than the ridges at each side. After the trench was completely dug, I carefully cleaned one wall with a spatula. I started sample collection immediately after the wall was cleaned, to minimize water loss from drying.

\section{Salt concentration measurements}

To determine the salt concentration in each sample, I followed the protocol tested in the laboratory setting and described in Sec. 3.2.4. For analysis of the samples from the first field campaign, I complemented gravimetric measurements with conductivity measurements of the individual samples conducted in the laboratory (also described in Sec. 3.2.4). Conductivity measurements proved to be less accurate. Additionally, conductivity measurements required a longer time to complete, which was an issue since I had to process a high number of samples. Therefore I did not do conductivity measurements of the samples from the second field campaign.

In addition to the conductivity measurements of the individual samples in the laboratory, I tried to measure soil conductivity directly in the extracted cores during the first days of the first field campaign. These measurements quickly proved to 
be very unreliable and heavily dependent on the type and water saturation of the soil. I therefore did not use try approach any further and no results from these measurements are included in the analysis of the data from the field campaigns.

\section{Pore water extraction}

During my second field campaign, I extracted pore water from depths below the maximum sampling depth reached with the corer or trench and from the top of the water table. From these pore water samples I got direct readings of the pore water salt concentration which are less prone to contamination by salt crystals from the surrounding soil. These pore water samples were later analyzed in the laboratory, using a vibrating-tube densimeter with a measurement accuracy of $5 \cdot 10^{-4} \mathrm{~g} / \mathrm{cm}^{3}$. The purpose of these measurements was to

- validate the salt concentration measurements from the soil samples and

- establish a very accurate reading of the background salt concentration.

To extract the pore water samples I used a custom-made tool consisting of a perforated, pointed metal tube with a small water reservoir at the bottom. The tool is depicted in Fig. 3.16. The holes could be covered by a filter, to prevent sand from entering the reservoir. After the tube was inserted into the ground at the desired depth, I waited for $1-5$ min until water had gathered gathered at the reservoir at the bottom of the tube. The volume of the reservoir was $\approx 2.4 \mathrm{~cm}^{3}$.

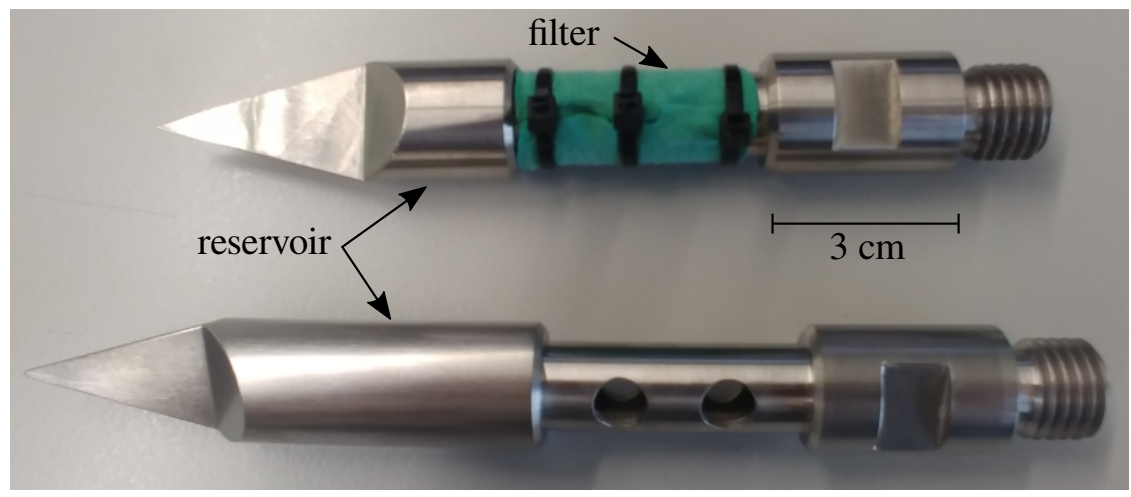

FIGURE 3.16: Tool to extract pore water from unconsolidated sand saturated with water.

\subsubsection{Pattern length scale characterization}

\section{Terrestrial laser surface scans}

To measure the dimensional pattern wavelength $\lambda$ and ridge height $h$ of the polygonal pattern at each site I visited, I recorded high resolution, three-dimensional point clouds of the surface relief using a terrestrial laser scanner (TLS). The scanner measures the time a laser beam originating from the scanner and pointing towards the 
surface at a known angle takes to be reflected from the surface. Distance to the surface is inferred and therefore height at the known position is inferred from the measured time.

In most cases the scanner was positioned at a height of at least $2 \mathrm{~m}$ above the crust and then recorded the surface relief in a circular sweep from a distance of about $1 \mathrm{~m}$ to the scanner up to a distance of about $50 \mathrm{~m}$. In principle the scanner can record the surface relief at even larger distances, but becomes increasingly prone to occlusion by higher surface features which lie in the path of the scanning beam. Additionally, resolution of the scan decreases with distance from the scanner as the number of points measured along a circle with a given radius stays constant, even if the radius increases. Conversely, the resolution is highest in the direct vicinity of the scanner. Therefore I aimed to position the scanner not more then $10 \mathrm{~m}$ away from the polygon I intended to sample, to record the pattern around the focal polygon with the highest possible accuracy.

At some sites it was possible to position the scanner on one of the gravel roads next to the site and therefore increase the vertical distance of the scanner to the crust to about $5 \mathrm{~m}$. This was desirable to reduce occlusion at larger scanning distances. At sites where this was possible, I aimed to position the scanner such that the focal polygon was in the center of the scanned area, which was a half-circle bounded by the gravel road.

I aimed to scan each site I sampled before beginning the sampling procedure and therefore disturbing the naturally grown crust. For several sites I also returned several days (during the same field campaign) to a year (in the second field campaign) later. In this way I was able to record long-term changes in the pattern length scale and short-term ridge growth.

\section{TLS Data processing}

Scans acquired by the TLS were subject to several post-processing steps described in detail elsewhere [154]. Here I summarize the main steps and describe their importance for my results. Post-processing steps include:

- Extraction of a $10 \times 10 \mathrm{~m}$ area of surface relief from the scan including the sampled polygon at each site.

- Gridding of the data points which were recorded on a radial coordinate system into Cartesian coordinates.

- Subtraction of a flat background surface as a correction factor for the artifacts introduced by the measurement instrument.

- Determination of continuous groups (features) of positive heights by searching for zero-up-crossings [155] from the baseline surface height.

- Feature height is calculated as twice the standard deviation of the detrended elevation within a feature. 
- Fourier transformation of the surface relief to find the frequency of features with a given length scale.

- Determination of $\lambda$ as the surface feature length scale that is larger than $90 \%$ of the length scales found in the relief $\left(90^{\text {th }}\right.$ percentile). The $90^{\text {th }}$ percentile is chosen as a measure that is robust to high frequency noise in the roughness and will reliably capture the dominant wavelength of the pattern.

- The dimensionless wavelength $\Lambda$ is then determined by dividing by the natural length scale $L=D / E$ defined in Eq. (2.13): $\Lambda=\lambda / L$.

- Determination of $h$ as the $90^{\text {th }}$ percentile feature height.

Due to the gridding into Cartesian coordinates, the best resolution of the surface relief is $1 \mathrm{~cm}$ in the horizontal and $0.3 \mathrm{~mm}$ in the vertical direction. This is sufficient to reliably determine both $\lambda$ as well as detect short-term ridge growth. Since I determined the wavelength of the patterns in Fourier space, I do not have access to individual polygon shapes. This makes it difficult to quantitatively compare the measured surface relief to planforms of convective dynamics. Nevertheless, a qualitative comparison based on photographs from the patterns is still possible.

\section{Crust thickness and ridge height}

While at the field sites, I measured crust thickness by removing the salt crust until reaching the sand below and then measuring the distance from sand to the top of the crust with a folding rule. While high precision TLS scans also capture ridge height, these scans are subject to occlusion by tall ridges, especially farther away from the position of the scanner. To complement the measurements with the TLS I also measured the height of several ridges per site using a folding rule.

\section{Timelapse imaging}

To investigate the rate of ridge growth and the most active areas of growth, I recorded time lapse images of crusts at Owens Lake. During my second field campaign I installed cameras (Ltl Acorn 5210A Trail Camera 940NM) at three sites. I chose locations for the cameras which were in close vicinity to the polygons I sampled. All cameras were positioned in managed areas which were rather wet during our visit. They therefore promised to show active crust growth as temperatures increased during the course of the year. One of the installed cameras is shown in Fig. 3.17.

I installed the cameras on tripods at a height of $150 \mathrm{~cm}$ above the crust and secured them with heavy rocks against the strong winds. The cameras were powered by batteries and recorded an image of a crust section of about $4 \mathrm{~m} \times 6 \mathrm{~m}$ in front of them every 30 minutes. I placed $10 \times 10 \mathrm{~cm}$ white, gray and black tiles in the camera's field of view as white balance and scale bar. Recording time was from January 16th to July 7th 2018. Images were recorded with a resolution of 12 megapixels. 
Cameras were equipped with an infrared flash, which enabled them to record images during the night as well. Cameras were also equipped with a temperature sensor, which recorded temperature with a precision of $\pm 1^{\circ} \mathrm{C}$. Since the temperature sensor was embedded in the camera's black casing, it overestimates temperatures during sunny days as the casing absorbs radiation and heats up. Date, time and temperature are encoded in the recorded images.

About 2 out of 3 images recorded by the cameras either failed to record completely or contained substantial digital artifacts, which rendered them useless for analysis. This might be due to the rather harsh climate conditions at Owens Lake. To compare images with comparable lighting conditions, I handpicked images that recorded properly for every day shortly after sunrise. Cameras stayed stationary during the whole period of recording and no realignment of images was necessary.

\subsubsection{Salt crust characterization}

In addition to the subsurface samples I am also interested in the chemical composition of the salt crust. In the laboratory I model the system using pure $\mathrm{NaCl}$. Nevertheless, the salts accumulated in a geological sink such as the Owens Lake or the Death Valley are a product of weathering of rock from the surrounding mountains [4] and are not expected to be pure. I do not expect the composition of salt species in the field to have a significant impact on the subsurface dynamics, since for the dynamics, the only important feature is expected to be the dependence of density on salt concentration. Nevertheless, differ-

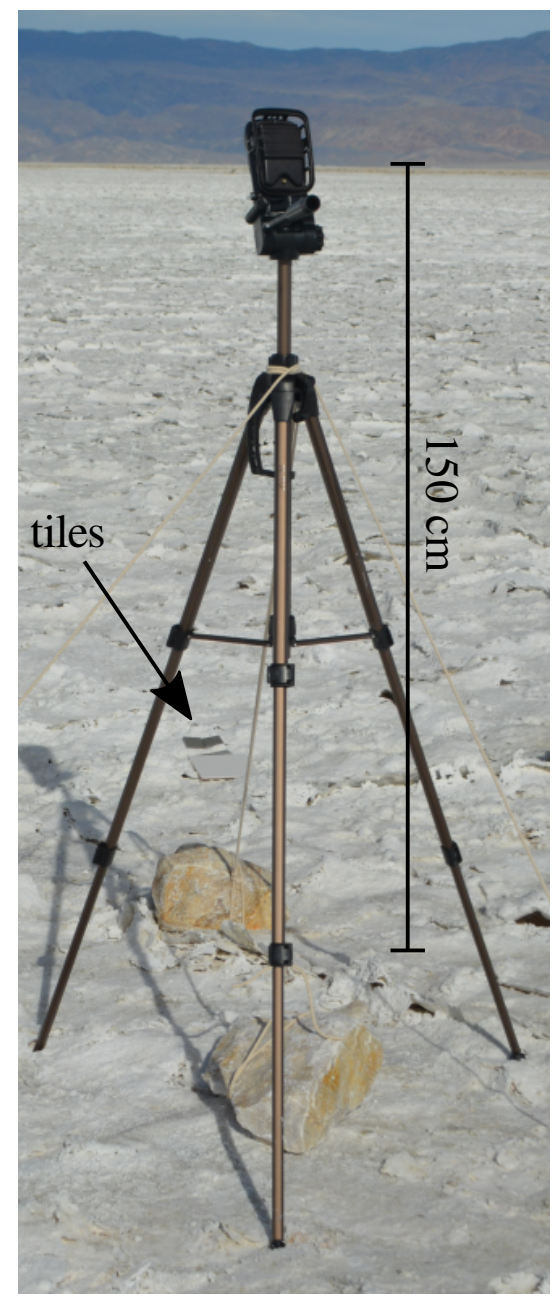

FIGURE 3.17: One of three cameras which were installed at Owens Lake in January 2018.

ent salts have different solubilities in water and their ions have different molecular diffusion coefficients. As salt concentration increases this leads to several effects:

- Salts precipitate at different maximum concentrations and may separate spatially during crust formation.

- The presence of more than one salt species in the solution can increase the maximum total salt concentration at saturation and therefore maximum possible density gradient. I approximate this effect to increase $\Delta \rho$ by up to $30 \%$. 
- Different ions have different diffusion coefficients and the effective molecular diffusion coefficient may be different from that of a mixture with a single salt. Depending on the type of diffusing ion, I expect this effect to decrease the molecular diffusion coefficient by up to a factor of three.

During my field campaigns, I collected samples from the salt crust near the polygons I investigated. The crust showed visually different regions, differing in both geometrical structure of the precipitated salts as well as color and humidity. For each site, I tried to collect samples from each of the different regions to analyze their mineral composition later in the lab. An example of a portion of crust with differently looking crust regions in close vicinity to each other is shown in figure 3.18.

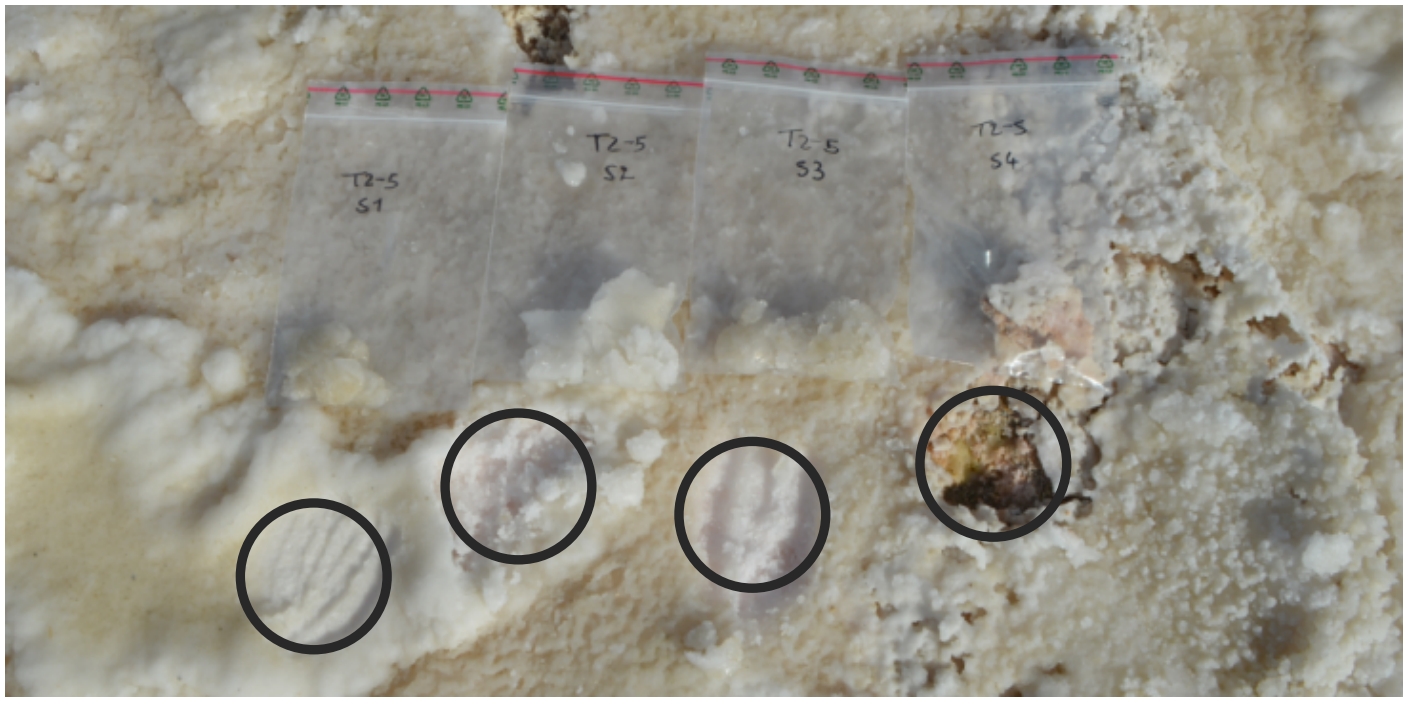

FIGURE 3.18: Exemplary illustration of different types of salt crust and sampling procedure. Sampling locations are indicated with black circles.

I do not expect different salt precipitation behavior to be of major consequence for my modelling of the subsurface dynamics, since there the salt is in solution. Nevertheless, I will consider the variety of ions in solution to arrive at a more accurate estimate of the diffusion coefficient of the system, as discussed in Sec. 4.3.4.

\section{X-ray diffraction analysis of crust minerals}

To characterize the minerals present in the pore water and crust, I analyzed them using quantitative X-ray powder diffraction (XRD). To prepare the samples, I followed the protocol detailed below:

- Similar to the protocol to measure salt concentration in the soil samples described in Sec. 3.2.4, sample mass was first measured, then samples were dried in an oven at $80^{\circ} \mathrm{C}$ for several days and then sample mass was measured again to measure the mass of the evaporated water. 
- Samples were mixed with $10 \mathrm{wt}$. $\% \mathrm{ZnO}$ (zinc oxide) powder as a known baseline. This is necessary to quantify amorphous parts in the sample later on.

- Samples were milled for $10 \mathrm{~min}$ in a McCrown micronizing mill to create a fine powder.

- Samples were back-loaded into $27 \mathrm{~mm}$ sample holders to preserve a random crystal orientation in the powder.

- Samples were scanned in an X-ray diffractometer and diffraction patterns recorded (details below).

- Minerals were identified using the X'Pert HighScore software (PANalytical).

- Mineral composition was quantified based on the Rietveld method using the software AutoQuan (Version 2.7.0.0).

For the XRD analysis I used a Philips X'Pert MPD PW 3040 diffractometer, equipped with a PW 3050/10 goniometer, divergence slit $0.5^{\circ}$, anti-scatter slit $0.5^{\circ}$, receiving slit $0.2 \mathrm{~mm}$, secondary graphite monochromator, mask $20 \mathrm{~mm}$, operating at $40 \mathrm{kV}$ and $30 \mathrm{~mA}$ with $\mathrm{Cu} \mathrm{K} \alpha$ radiation. The range $2 \theta=4-69.5^{\circ}$ was scanned with a step width of $0.02^{\circ}$. The counting time was $10 \mathrm{~s}$, sample spinning was at $1 \mathrm{rps}$.

\section{Spectrometry analysis of crust minerals}

To confirm the mineral quantifications measured by XRD, I used inductively coupled plasma optical emission spectrometry (ICP-OES) to classify the ions in some of the pore water samples. Minerals from the pore water samples were first dried as described above (since they were part of the gravimetric salinity determination). Samples were then re-dissolved in a known amount of water for measurement with the ICP-OES.

All elements were analyzed by an Agilent 5100 VDV ICP-OES. Ba, Ca, K, Na, Sr, were measured in radial view mode all other elements in axial view mode. Standardization was done by using five matrix matched multi element calibration solutions and a blank solution. All solutions contained $1.56 \mathrm{~mol} / 1 \mathrm{HNO}_{3}$. The mean of six blank measurements was subtracted from each calibrated value. The detection limit was calculated as $3 \sigma D_{\text {BLANK. }}$.

\section{Biological sample components}

As visible in Fig. 1.5 (f) and (g), the salt crusts and brine pools at Owens Lake show an intense red color. This is due to a microorganism, the algae Dunaliella Salina, which produces high amounts of $\beta$-Carotene [40]. As there is considerable interest in modeling algae and their behaviour in my research community, I agreed to sample water and salt crust from several sites showing signs of Dunaliella Salina presence, 
as depicted in Fig. 3.19. The samples are now being cultivated in the algae collection of the Georg-August-University Göttingen. Images of some of the cultivated microorganisms are shown in Appendix C.
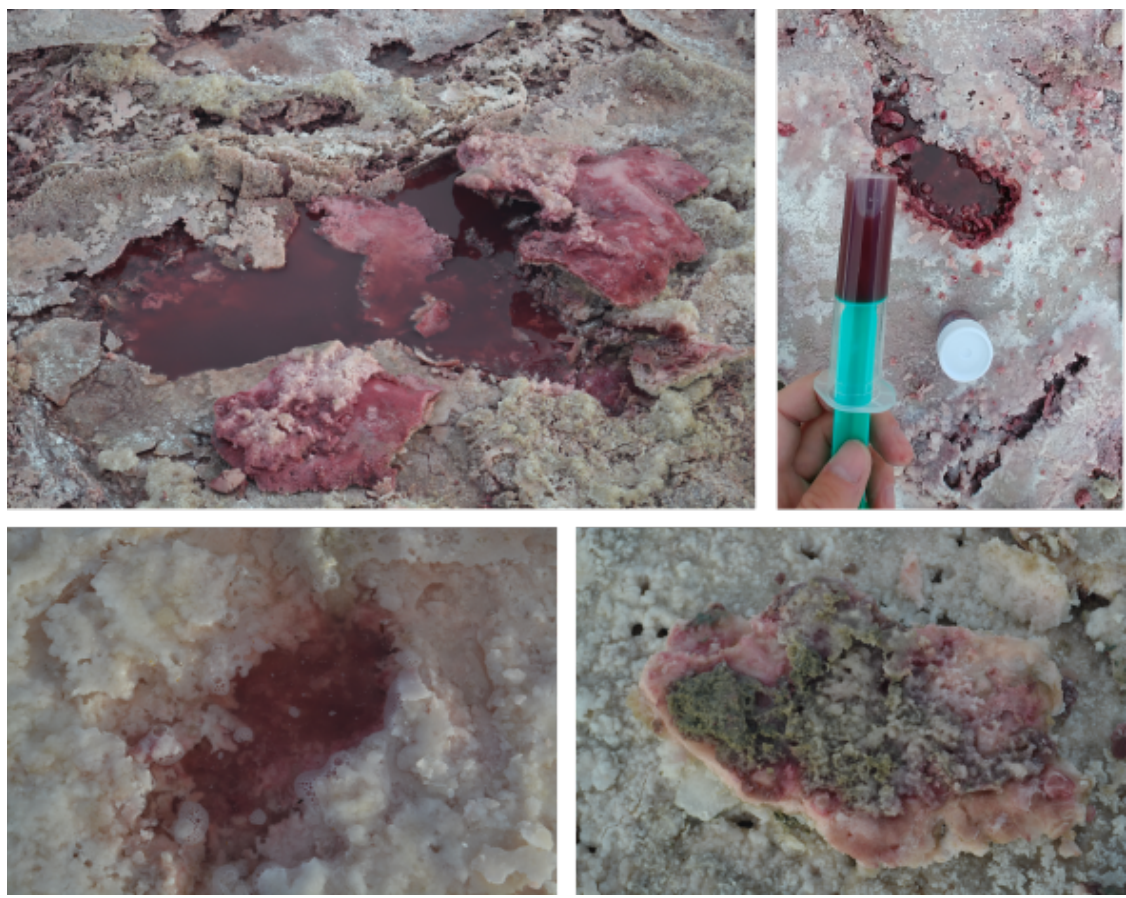

FIGURE 3.19: Sampling of red Dunaliella Salina from brine and salt crust at several sites at Owens Lake, CA.

Salt extracted from the collected soil samples also showed a beige to brown color, an indication for a significant proportion of biological components in the samples. To quantify the biological portion of samples I heated the soil and salt samples to about $1000^{\circ} \mathrm{C}$ to burn off all biological matter. I continued the burning until the samples visibly changed color: salt samples turned completely white while sand samples changed to a lighter shade of beige or brown. This took approximately $10 \mathrm{~min}$ per sample. I then weighed the samples to quantify the lost mass and concluded that this mass could be attributed to biological material.

A temperature range between $200^{\circ} \mathrm{C}$ and $1150^{\circ} \mathrm{C}$, in which all biological components are burnt off of clay was identified by [156]. The boiling points of silicon as well as sodium chloride and sodium sulfate, which are the main components of the samples (see Tab. B.3), lie well above $1400^{\circ} \mathrm{C}$. My chosen burning temperature of $1000^{\circ} \mathrm{C}$ is at the upper limit of the burning range of organic components from clay yet well below the boiling points of the other components. Therefore it is warranted to assume, that the burning procedure successfully removed all biological sample components and that all of the lost mass can be attributed to organic components. 


\subsubsection{System parameters}

\section{Permeability}

To determine the permeability of the soil at my field sites, I measure the grain diameters in the sand samples using a laser particle sizer (LPS), as described in Sec. 3.2.2. Grain diameter distributions vary depending on the depth at which the sample was taken, which is indicative for a sedimentation process by different flooding events.

As described in Sec. 3.2.2, I use the Sauter diameter of the porous medium as characteristic length scale. At all sites I took samples from at least down to a depth of $\approx 50 \mathrm{~cm}$ below the crust. I want to arrive at a measure of the characteristic length scale which is comparable between all sites. Therefore I only use samples which originate from a depth at or above $50 \mathrm{~cm}$ for the calculation of $d_{s}$. I calculate $d_{S, i}$ from the LPS measurements for each sample $i$ individually following Eq. (3.14). I then calculate the permeability of the site using the average Sauter diameter $\left\langle d_{S}\right\rangle=\sum_{i} d_{S, i}$ as defined in Eq. (3.16).

Using only the samples originating from the top $50 \mathrm{~cm}$ of soil is warranted, since the initial instability occurs at the top boundary of the porous medium. Therefore the permeability close to the top boundary is more important to determine, whether the situation is unstable and to set the wavelength of the instability.

\section{Evaporation rate}

It was beyond the scope of my work to measure evaporation rates $E$ at the field sites during the two field campaigns myself. This was due both to limited time and equipment as well as to the fact, that we visited the sites in winter to be able to work on the salt flats in moderate temperatures. I have evidence (see Sec. 4.7.2) that the main growth season for salt polygons is in late spring and summer, therefore evaporation rates measured in winter would not have been representative.

I need an estimate of $E$ to calculate the Rayleigh number and natural time and length scales as defined in Eq. (2.13). Luckily, since Owens Lake is the subject of intense research efforts connected to the dust control project, evaporation rates under different circumstances have been characterized in the past [44, 37].

Evaporation rates $E$ from concentrated brine at Owens Lake have been measured to be around $0.5 \mathrm{~mm} /$ day by [44] using open water pans embedded in the salt crust during summer. [37] reports an $E$ between 0.05 and $0.3 \mathrm{~mm} /$ day from brine covered with a thin salt crust. Differences can be explained by the concentration of salt in the brine, which significantly influences the activity of the saline solution [157, 158] as well as the presence of a salt crust, which [37] reports to inhibit evaporation.

Here I choose to use the average of the two reported evaporation rates, namely $E=0.4 \pm 0.1 \mathrm{~mm} /$ day.

For Badwater Basin, there are measurements of evaporation rates from different types of soil as well: using an energy budget and eddy correlation method, [45] estimated evaporation rates at Badwater Basin to be $E \approx 0.31 \mathrm{~mm} /$ day and 
$E \approx 0.14 \mathrm{~mm} /$ day for salt encrusted playa for years with and without flooding respectively. Here I use the evaporation rate from years where flooding occured, because it more closely resembles the circumstances at the onset of instability and beginning of crust formation.

For Bristol Playa, I did not find records of evaporation rate measurements. I therefore use the evaporation rate measured at Owens Lake, since the general weather conditions at Bristol Playa are more similar to Owens Lake than to Badwater Basin.

\section{Weather at the field sites}

Ambient circumstances such as temperature, air humidity and air flow i.e. wind speed over the crust surface are important factors that can explain diurnal, seasonal and yearly differences in evaporation rates. Publicly available weather records for Lone Pine, a town approximately $8 \mathrm{~km}$ north of the shore of Owens Lake, report average wind speeds of approximately $10 \mathrm{~km} / \mathrm{h}$ [42]. Peak temperatures in July are approximately $36^{\circ} \mathrm{C}$ and relative humidity is in the range of $0-10 \%$ [42]. For the sites visited during the two field campaigns, personnel responsible for the dust control project informed me that the sites had been flooded between one and two years prior to our visits. This sets the estimate of the age of the polygons we encountered to a maximum of two years.

Additionally, two days before the start of our second field campaign there was an unusually strong precipitation event at Owens Lake. According to local researchers, the surface of the dry lakebed received about $2.5 \mathrm{~cm}$ of precipitation over approximately 24 hours. The surface was also visibly wet during the first three to four days of sampling.

For Furnace Creek, a town about $25 \mathrm{~km}$ north of Badwater Basin, publicly available weather records report average wind speeds similar to Owens Lake [43]. Peak temperatures in July are around $46^{\circ} \mathrm{C}$ and therefore considerably higher than at Owens Lake, whereas relative humidity is in the range of $0-30 \%$ [43]. Death Valley including Badwater Basin was flooded in October 2015 (roughly 14 months prior to the sampling campaign) as well as in September 2017 and July 2018 (both floods did not affect sampling). The years 2014-2018 showed an increased frequency of heavy flooding compared to the long-time average. As with Owens Lake, I assume that while ridges might not be wiped out completely during a flooding event, ridge height will be greatly diminished as the salt crust dissolved. Therefore I can at least estimate the time-scale on which ridges grow and are wiped out again based on the measured ridge height and the frequency of flooding events - which is in the range of years.

Temperature and humidity are the main parameters (next to salt concentration and air movement) controlling evaporation from the salt pan. To get at least an indirect estimation of the influence of ridges on the micro-climate at crust level, I embedded sensors in several ridges and tracked temperature and humidity over several days. The measurements from within ridges can be compared to measurements of 
temperature and humidity recorded from sensors placed in the centers of polygons. For temperature and humidity measurements, I used HiTemp140 and RHTemp1000IS data loggers, which measured temperatures and relative humidity with a precision of $\pm 0.01^{\circ} \mathrm{C}$ and $\pm 0.1 \%$ respectively.

\subsubsection{Field site geology}

In addition to the measurable parameters of the system I was also interested the general geological, geomorphological, hydrological and biological background of the field sites and resident microorganisms. I wanted to get a more complete picture of the type of location that supports the emergence of salt polygons. Therefore I conducted a few additional measurements as well as a thorough literature search of the geological and anthropological history of my field sites. The latter is summarized in Sec. 1.2.2, the former is described below.

\section{Owens Lake geology}

The Owens Lake basin is bounded by the Sierra Nevada fault zone in the West and the Inyo Mountain fault zone in the east. [3] The Owens Valley graben is deepest below Owens Lake: the valley fill reaches a depth of about $2.4 \mathrm{~km}$ above the bedrock [3]. The valley fill below the dry lake itself consists of moderately to wellsorted layers of sand with grain sizes that range between clay, fine to coarse sand and gravel [3]. This is important to note, as for the purpose of modeling the flow dynamics in the porous medium - which occur on a length scale of meters - the vertical extent of the porous medium can be assumed to be infinite.

All sampling locations at Owens Lake were situated in the area of the fluvial and lacrustine deposits, as indicated in Fig. 3.20. The dry lake is framed by younger and older alluvial fan deposits. A much more detailed description of the geology of the Owens Valley can be found at $[3,159,160]$. The dry lake's surface is subdivided into cells on which different dust control measures are implemented. As indicated in Fig. 3.13, I focused my sampling efforts on the brine cells in the North and South of the lake. I sampled a total of 21 polygon cross sections in 11 different management cells.

\section{Badwater Basin geology}

Badwater Basin is a geological sink and the deepest point on land in North America, about $86 \mathrm{~m}$ below sea level [161]. Similar to Owens Lake it is subject to very infrequent precipitation events and evaporation from the playa far outweighs precipitation [45]. Water flows into the basin from the surrounding mountains, carrying minerals which accumulate in the basin [161].

The geology of the Badwater Basin and the surrounding Death Valley is described in much detail elsewhere $[161,159]$ but similar to Owens Lake, it also exhibits a very deep bed of unconsolidated valley fill, on which the crust rests. 


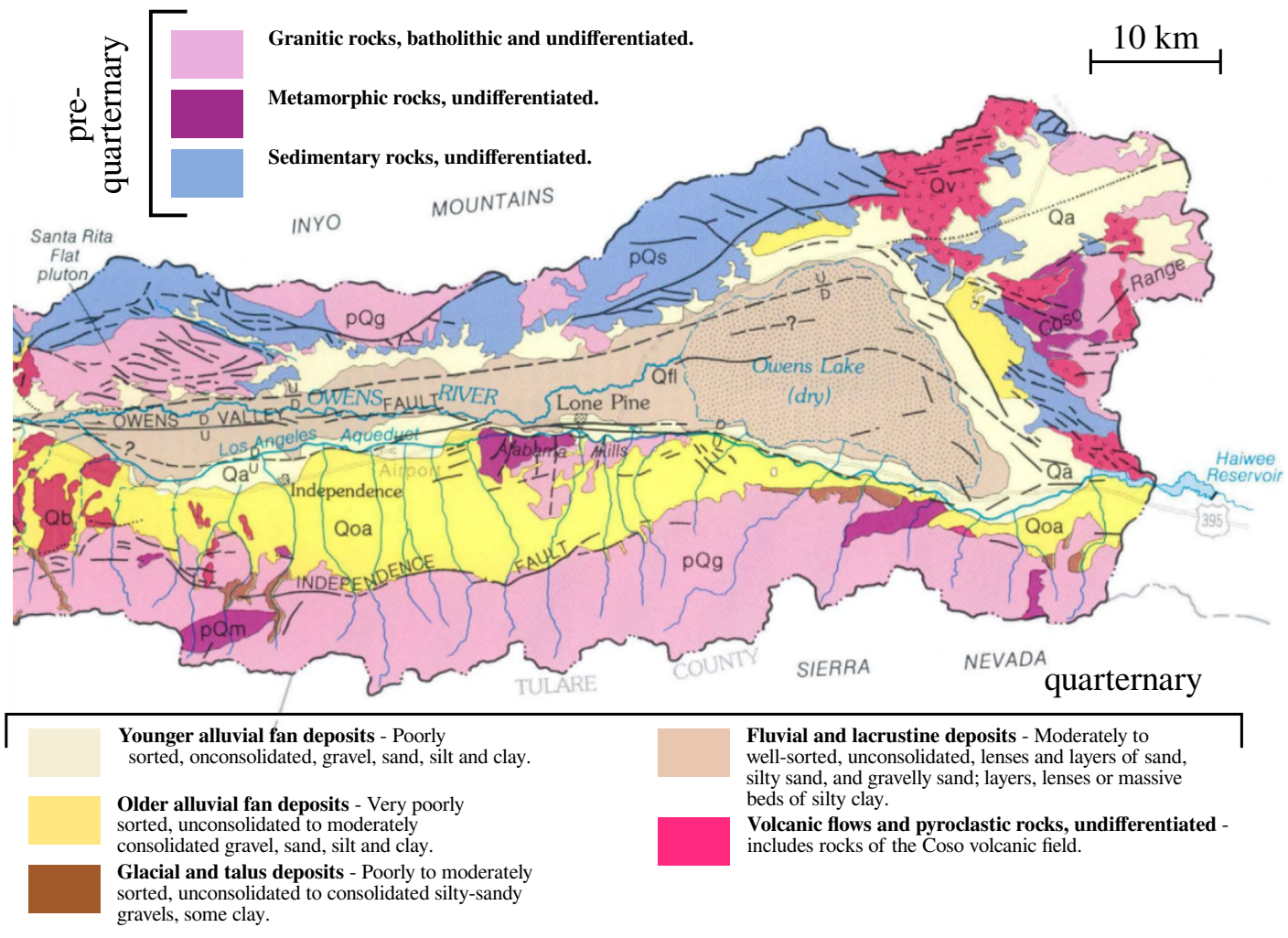

FIGURE 3.20: Geologic map of Owens Valley, adapted from [3].

Since all of Death Valley is a national park, I had to adhere to a restricted sampling protocol. Approval was gained from the US national parks service to sample five polygon cross sections in total up to a depth of approximately $50 \mathrm{~cm}$. I sampled polygons in an area about $500 \mathrm{~m}$ south of the main tourist pathway entering the salt flats from the east, which was assigned to me by the national parks service. I picked this area to minimize the possibility that polygons were disturbed by tourists trampling them.

I sampled two polygons about $100 \mathrm{~m}$ inwards and parallel to the "shore" of the salt flats as depicted in the map of the area in Fig. 3.14. Additionally I sampled three polygons at a distance of about $200 \mathrm{~m}, 300 \mathrm{~m}$ and $400 \mathrm{~m}$ distance inwards from the shore to, be able to detect any possible changes in polygon size with distance to the shore.

Next to the sites I visited for sampling purposes, I also visited the iconic polygons at the tourist entrance and took photographs. Polygons there seemed to have a different history then the ones we sampled, since they exhibited both sandy brown as well as pristine white ridges and a smaller length scale. Both are indicators for different ridge age: a smaller length scale points to a pattern before coarsening took place (see Sec. 4.5 for details), whereas the brown color indicates that the polygons have been covered by fine sediment over time, either carried there by wind or during a flooding event. Exemplary polygons from the sampling sites are shown in Fig. 3.21 (a) and (b) whereas panels (c) and (d) show polygons from the tourist site. 

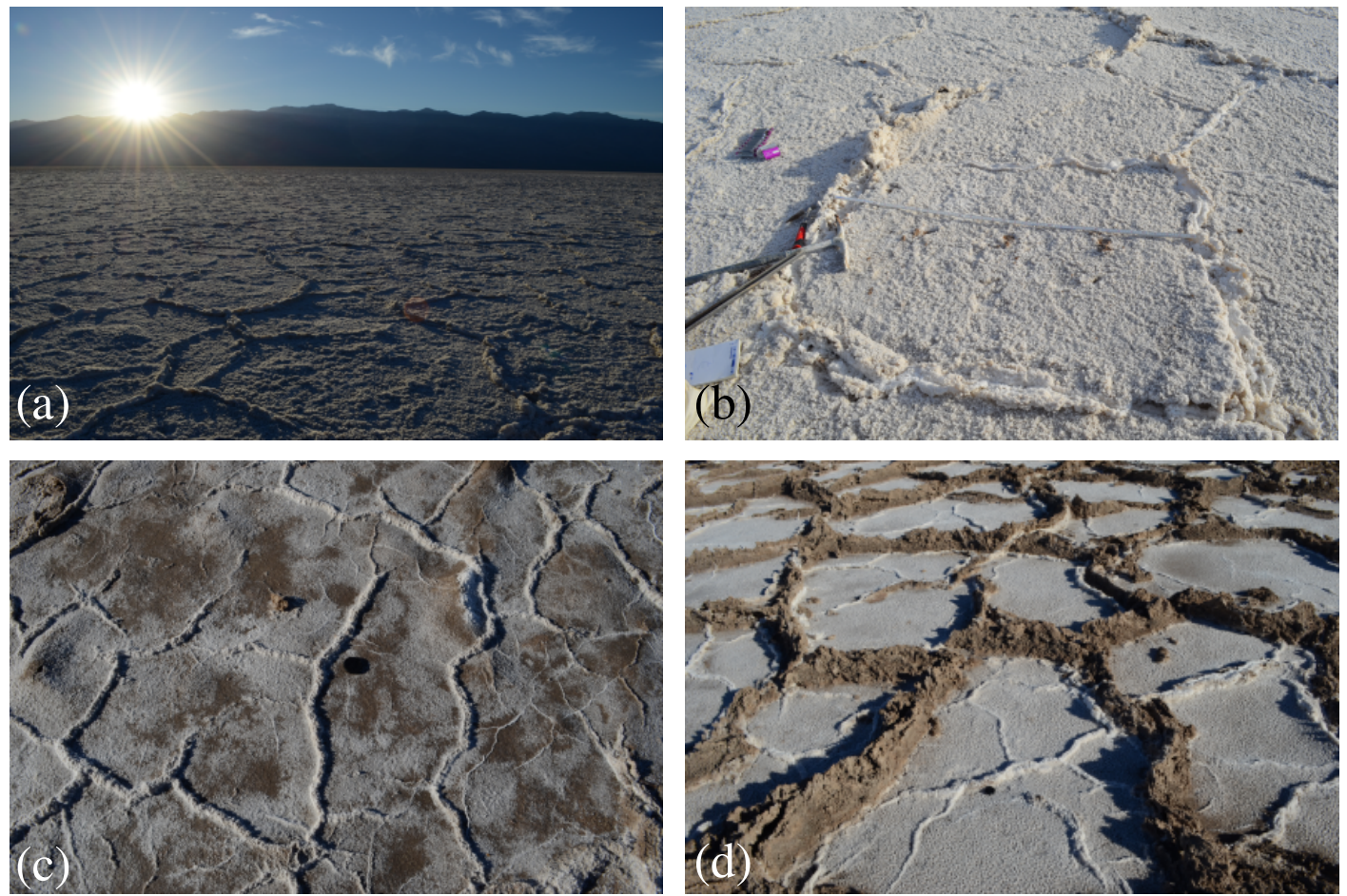

FIGURE 3.21: Exemplary polygons encountered at Badwater Basin during my field campaign in 2016. (a) and (b) polygons about $500 \mathrm{~m}$ south of the Badwater Basin tourist entrance. (c) small (about $1 \mathrm{~cm}$ high) ridges and (d) old (brown) ridges from before the 2015 flash flood interwoven with younger (white) ridges at the Badwater Basin tourist entrance.

\section{Bristol Playa and Soda Dry Lake}

Bristol Dry Lake is a salt lake in south eastern California. It has a similar geological setting as Owens Lake and Badwater Basin [162], and also shows similar polygonal structures. Nevertheless the area is subject to heavy land-moving efforts from the neighboring salt mine. I sampled one polygon at Bristol Playa during the field campaign in 2016 and scanned the surface. During the sampling it became obvious that the soil had been recently disturbed, since large parts of broken crust and large salt crystals had been plowed under the surface. I determined that the samples would not yield any reliable results.

During my field campaign in 2016 I also visited Soda Dry Lake in the Mojave Desert, California. Soda Dry Lake shows similar geological and meteorological characteristics as the other sites. Therefore it was a promising research location, since the research station in Zzyzzx is located in close vicinity and could have supported any research efforts. Nevertheless on my short scouting mission there I was not able to locate any polygons.

\subsubsection{Summary}

I introduced measurement methodologies and protocols to study buoyancy-driven convection as well as the polygonal salt ridge patterns in the field. Observables of 
interest are the salinity distribution in the soil below the patterns, which I measure by taking samples from cross-sections below polygons and the surface relief of the salt crust, which I measure using a terrestrial laser scanner. Furthermore, I introduce methodologies to analyze the mineral composition of the salt crust. I also describe the knowledge that exists in literature about the weather conditions and the geology of the various field sites I visited. 



\section{Chapter 4}

\section{Results}

\subsection{Introduction}

In this chapter I present how I applied the methods described in the previous chapter to investigate, whether the formation of polygonal ridge patterns in salt deserts is driven by convection. The organization of this chapter follows my presentation of evidence for my theory for the driving mechanism that causes salt polygons along several lines of argumentation:

Instability: In Sec. 4.2 I analytically determine the onset of instability and ensuing convection dependent on the Rayleigh number and the dominant mode of growth. Once I have determined the critical Rayleigh number, I measure the system parameters which determine the Rayleigh number in the experiment and field and, subsequently, find that the systems I investigated are expected to be unstable.

Density stratification: In Sec. 4.3 I show evidence for a vertical salinity gradient that builds up in the experiment and in the field and investigate the properties of this gradient using numerical simulations.

Convection and co-location: In Sec. 4.4 I present direct hydrological evidence for salinity gradient driven convection from salt concentration profiles collected both in the laboratory and the field. The concentration profiles also clearly show the colocation of the downwellings with the patterns at the surface.

Wavelength selection: In Sec. 4.5 I explain the length scale of the observed patterns and their similarity across different locations and show that the wavelength measured in the field is consistent with the experiments and numerical simulations, if coarsening is taken into account.

Feedback: In Sec. 4.6 I introduce the necessity of a feedback mechanism between subsurface dynamics and salt crust and investigate it in numerical simulations. I also show circumstantial evidence from the field which supports the existence of 
such a feedback mechanism.

Crust growth and timescale: In Sec. 4.7 I present direct and indirect measurements of crust growth, allowing for an estimation of the growth rate of salt ridges. I also compare the age of the systems found in nature as determined by their coarsening state to their natural time scale and the natural growth cycle set by flooding events.

\subsection{Instability}

\subsubsection{Linear stability analysis}

The following is reproduced in an abbreviated form from the master's thesis of Marcel Ernst [110], who investigated the theoretical and numerical background of the salt playa problem in close collaboration to my work and under my regular supervision.

If we let the system described by Eqs. (2.15), (2.17) and (2.16) evolve in time, a salinity gradient builds up below the surface. To determine, whether this stratified configuration can become unstable and drive convection, We employ a linear stability analysis of the governing equations. Here we only consider scenario (3), the "salt pan" boundary condition with constant flux at the top boundary: a sandy soil saturated with salty water and covered with a uniform salt crust and homogeneous evaporation. The linear stability analysis for scenario (1), the "salt lake" boundary condition, where pressure at the top boundary is constant, is similar and can be found in [58, 110]. See Sec. 2.6 for details on the selected boundary conditions.

We omit the linear stability analysis for scenario (2) - constant flux and nonuniform salinity -, since it is expected to only be present in nature for a very short time, before a salt crust forms. We were not able to perform a linear stability analysis for scenario (4) - non-uniform flow rate - analytically, since in this scenario the top boundary condition is dependent on the horizontal direction. We still consider this scenario in the numerical simulations.

We start by considering small perturbations to the salinity, pressure and velocity relative to the steady state of the system where $S^{0}=e^{Z}$ :

$$
\widetilde{S}=S-S^{0}, \quad \widetilde{P}=P-P^{0}, \quad \widetilde{U}=\boldsymbol{U}-\boldsymbol{U}^{0}=\left(\begin{array}{c}
\widetilde{U}_{X} \\
\widetilde{U}_{Z}
\end{array}\right) .
$$


We insert the perturbed quantities into equations (2.15) to (2.16) and linearize, arriving at the governing equation for the perturbations

$$
\begin{aligned}
\nabla \cdot \widetilde{\boldsymbol{U}} & =0 \\
\frac{\partial \widetilde{S}}{\partial \tau}+\frac{\partial \widetilde{S}}{\partial Z}+\widetilde{U}_{Z} \frac{\partial S^{0}}{\partial Z}-\nabla^{2} \widetilde{S} & =0 \\
\nabla \widetilde{P}+\operatorname{Ra} \widetilde{S} \boldsymbol{U}^{0}+\widetilde{\boldsymbol{U}} & =0 .
\end{aligned}
$$

The second and third term in Eq. (4.3) only depend on $Z$, since the steady state solution only depends on the vertical direction. We eliminate pressure perturbations $\widetilde{P}$ and horizontal velocity perturbations from Eq. (4.4), rearrange Eq. (4.3) and arrive at

$$
\begin{array}{r}
\nabla^{2} \widetilde{U}_{Z}+\operatorname{Ra}\left(\frac{\partial^{2} \widetilde{S}}{\partial X^{2}}+\frac{\partial^{2} \widetilde{S}}{\partial Y^{2}}\right)=0 \\
\widetilde{U}_{Z}=\left(\frac{\partial S^{0}}{\partial Z}\right)^{-1}\left(\nabla^{2} \widetilde{S}-\frac{\partial \widetilde{S}}{\partial Z}-\frac{\partial \widetilde{S}}{\partial \tau}\right) .
\end{array}
$$

For the salt pan boundary condition we know that $S(X, Y, 0, \tau)=1$ and can set $\widetilde{S}(X, Y, 0, \tau)=0$. Furthermore $U_{Z}(X, Z=0, \tau)=1$ and therefore $\widetilde{U}_{Z}(X, Z=0, \tau)=$ 0 . Additionally, the vertical velocity perturbation must vanish for $Z \rightarrow-\infty$. Since we know the steady state solution for the salinity $\frac{\partial S^{0}}{\partial Z}=e^{Z}$, we can conclude from Eq. (4.6) that for $Z \rightarrow-\infty$ the term $\nabla^{2} \widetilde{S}-\frac{\partial \widetilde{S}}{\partial Z}-\frac{\partial \widetilde{S}}{\partial \tau}$ has to decay faster than $e^{Z}$ to satisfy $\widetilde{U}_{Z}(Z \rightarrow-\infty) \rightarrow 0$.

We employ the approach described by [163] and decompose the salinity perturbation $\widetilde{S}(X, Y, Z, \tau)$ into a most unstable vertical perturbation $F(Z)$ and a nondimensional growth rate $\Omega$

$$
\widetilde{S}(X, Y, Z, \tau)=F(Z) \Phi(X, Y) \exp (\Omega \tau)
$$

with the function $\Phi(X, Y)$ satisfying

$$
\frac{\partial^{2} \Phi(X, Y)}{\partial X^{2}}+\frac{\partial^{2} \Phi(X, Y)}{\partial Y^{2}}+a^{2} \Phi(X, Y)=0
$$

Here $a$ is the characteristic wavenumber of the perturbation in horizontal direction.

For growth rates of $\Omega<0$, the initial perturbation decays and the system returns to the steady state solution. For values of $\Omega>0$, the initial perturbation grows and the system is unstable. we insert the salinity perturbation from Eq. (4.7) into Eq. (4.4) while substituting $\widetilde{U}_{Z}$ with Eq. (4.6) and arrive at an eigenvalue problem for $F(Z)$,

$$
\left(\partial^{2} Z-a^{2} \partial Z\right) e^{-Z}\left(\partial^{2} Z-\partial Z-a^{2}-\Omega\right) F(Z)=a^{2} \operatorname{Ra} F(Z) .
$$

Solving the eigenvalue problem gives a relation between the Rayleigh number $\mathrm{Ra}$, the growth rate $\Omega$ and the horizontal wavenumber $a$. This was done by Marcel Ernst 
in within the scope of his master's thesis (see [110] for details of the calculation).

The linear stability analysis for the salt lake scenario, with constant pressure at the top boundary instead of uniform flow rate (details also given in [110]) finds a slightly different Rayleigh number and wavenumber at neutral stability. The critical Rayleigh numbers and wavenumbers for both scenarios are shown in Tab. 4.1. Eliminating the $Y$-direction in the calculations does not change the results of the lin-

\begin{tabular}{c|c|c} 
Boundary condition & $\mathrm{Ra}_{c}$ & $a_{c}$ \\
\hline (1) constant pressure & 6.954 & 0.429 \\
(3) uniform flow rate & 14.35 & 0.759
\end{tabular}

TABLE 4.1: Critical Rayleigh number and wavenumber for the "salt lake" and "salt pan" boundary conditions corresponding to scenarios constant pressure and constant flux described in Sec. 2.6.

ear stability analysis. Therefore the values we find for the critical Rayleigh number and wavenumber are also valid for the two-dimensional and quasi two-dimensional settings of the simulation and experiments respectively.

Next to finding the conditions necessary for the onset of convection, this semianalytical approach allows for the calculation of the expected growth rates of the most unstable mode as well as the neutral stability curve for Rayleigh numbers away from onset. This is important, since the systems observed in nature have Rayleigh numbers far greater than $\mathrm{Ra}_{c}$ and therefore may express wavenumbers different from $a_{c}$.

Neutral stability curves and most unstable mode have been determined in [110] up to $\mathrm{Ra}=0-3000$ and $a=0-12$ within the scope of his master's thesis. They are shown in Fig. 4.1 for both scenario (1) and (3). In the following I will always compare my findings to the theoretical predictions corresponding to the salt pan scenario, since it is the scenario I found and investigated in the field, experiment and simulations. In the following, I will first calculate the Rayleigh number for the field sites I investigated, followed for an analysis of the Rayleigh number in the experimental setups I used and determine the stability of the respective systems based on the theoretical prediction for $\mathrm{Ra}_{c}$.

\subsubsection{Instability under field conditions}

To calculate Ra under field conditions and determine, if the system is unstable, I measure or approximate the various constants entering the Rayleigh number

$$
\mathrm{Ra}=\frac{\kappa \Delta \rho g}{E \mu \phi}
$$

I approximate the evaporation rate for Owens Lake and Bristol Playa as $E=0.4 \pm 0.1 \mathrm{~mm} /$ day and for Badwater Basin $E=0.31 \mathrm{~mm} /$ day (as described in Sec. 3.3.4) and following [44, 37, 45]. Furthermore, I assume viscosity and gravity to be constant at $\mu=10^{-3}$ Pa s [109] and $g=9.8 \mathrm{~m} / \mathrm{s}$. Permeability and porosity are 


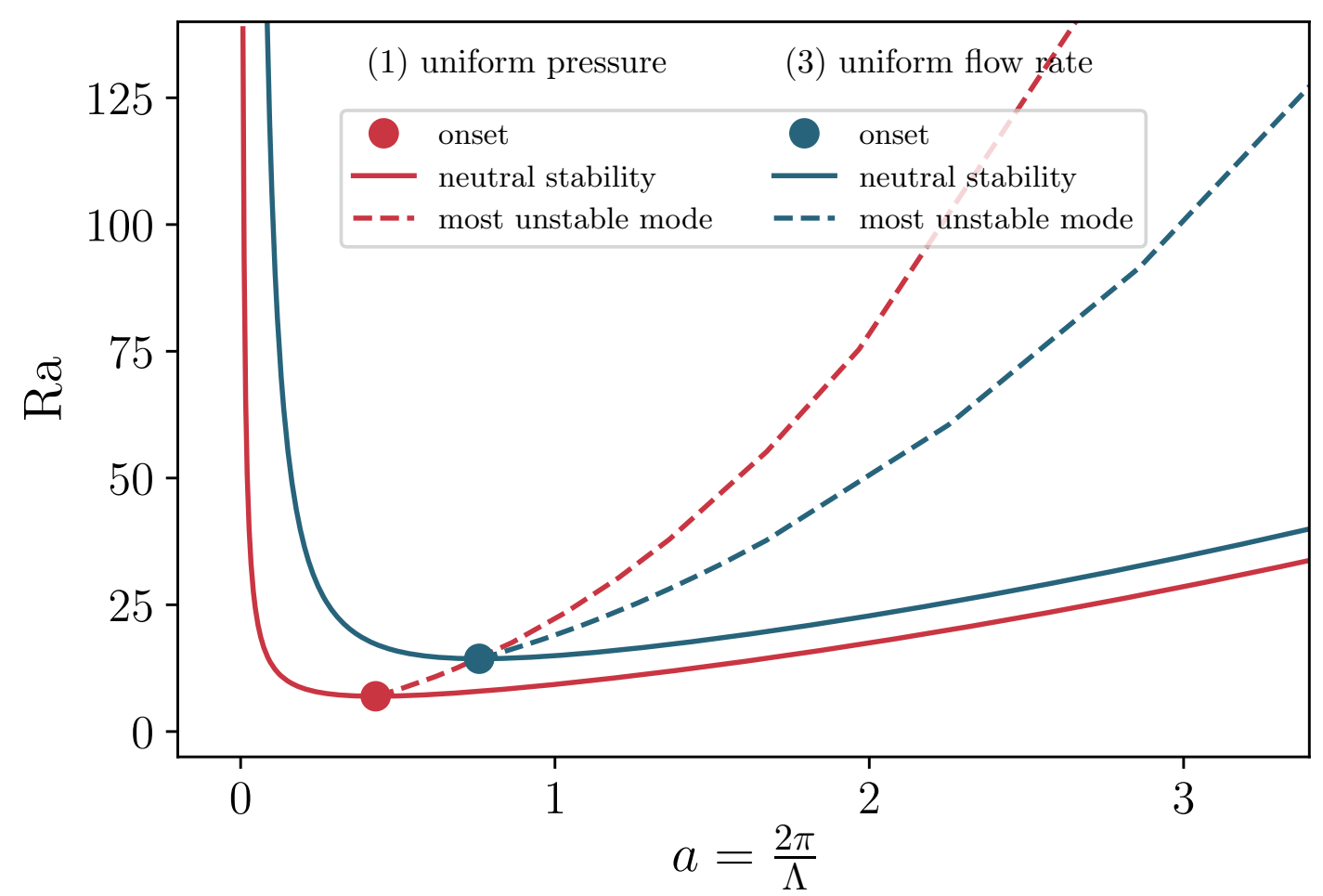

FIGURE 4.1: Onset of instability (dots), neutral stability curve (solid line) and most unstable mode (dotted line) for scenario (1), the "salt lake" condition at uniform pressure (red) and scenario (3), the "salt pan" condition at uniform flow rate (blue).

calculated based on the Sauter diameter (see Sec. 3.2.2), which I measured for the porous medium. I describe the results of measurements of the density difference $\Delta \rho$ and characteristic length scale below.

\section{Site nomenclature}

For the field sites located at Owens Lake, I use the labels referring to surface management cells related to the dust control project (described in Sec. 1.2.2). These labels either refer to managed cells or to unmanaged areas in direct vicinity of a managed cell. Labels always start with "TX-Y", where $X$ is a number and $Y$ is a letter. The numbers refer to water outlet taps along the main water pipeline that crosses the lakebed south to north and is used to irrigate management cells. Low tap numbers start in the south and increase northwards. The letters are "A", "W" and "S" and stand for "Addition", "South" and "West". They refer to additional sub-regions within a management cell.

For some sites I investigated more than one polygon. This is indicated in brackets next to the site label, e.g. T27-A (3) is the third polygon investigated at site T27-A, which corresponds to the "Addition" region of the management cell next to the $27^{\text {th }}$ water tap. I also use the numbering for the sites visited at Badwater Basin. These labels make my general findings about the mineral and soil composition relatable to other research done within the scope of the dust control project. 
The site sampled at Bristol Playa will be part of the tables and figures I show in the following analysis. Nevertheless, I will not interpret the results from Bristol Playa separately, since it is only one data point and any statements about trends as compared to the sites at Owens Lake and Badwater Basin will not be reliable.

\section{Grain size, permeability and porosity}

To calculate the characteristic length scale for the porous medium in each sample, I first determine the grain size distribution as described in Sec. 3.2.2. I note that for the display of data from the field campaigns and the experiment, sample depth is indicated in distance from the surface and $|z|$, since the $z$-direction is defined to point in the opposite direction as gravity.
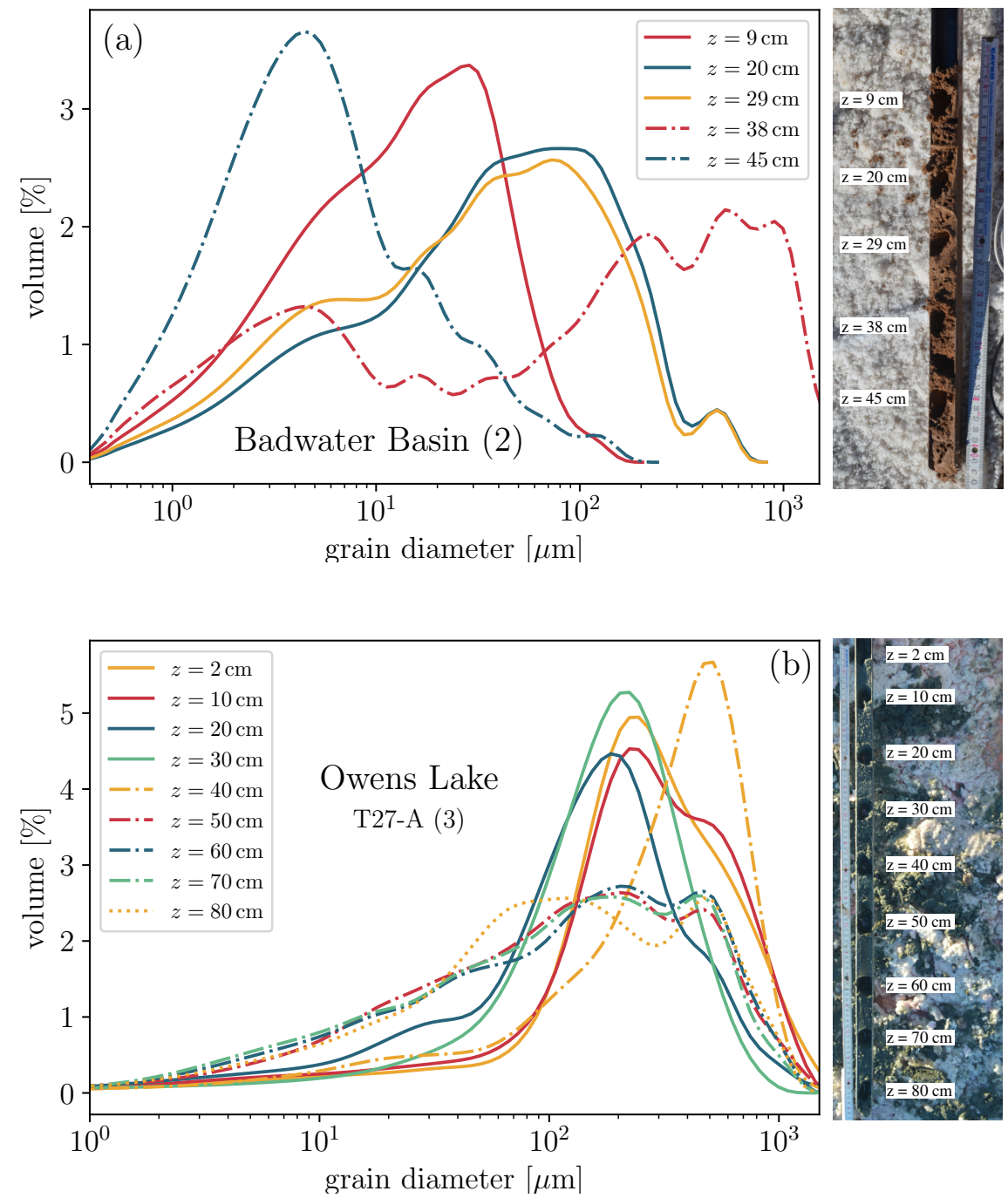

FIGURE 4.2: Exemplary grain size distributions for samples at different heights $z$ measured by LPS for (a) Badwater Basin and (b) Owens Lake. Images to the right show the original cores from which samples were collected. 
Grain size distributions were measured with a laser particle sizer (LPS). They vary widely for different field sites and different samples, with distribution maxima between $10^{0} \mu \mathrm{m}$ and $10^{3} \mu \mathrm{m}$. Grain size distribution is different for different sites and also depends on the depth at which they were sampled. Many samples show characteristic multi-modal grain size distributions, indicative of a fluvial deposition process [164] with different flow speeds, as shown in Fig. 4.2 for (a) Badwater Basin and (b) Owens Lake. Grain sizes tended to be smaller near the surface and at greater depths. Small grain sizes near the surface could originate from dust deposition by wind, since especially Owens Lake is subject to strong winds and known to emit a significant amount of dust [32].

Next to the exemplary grain size distributions I show the corresponding cores and sampling locations along the core. Especially sites at Owens Lake showed pronounced and visible layers differing both in color as well as in grain size and water content. Qualitative descriptions of the layers following the Udden-Wentworth scale [136] for each site are given in Appendix A.

I calculate the Sauter diameter $d_{S}$ based on the grain size distribution for each site as described in Sec. 3.2.2. To determine the permeability $\mathcal{K}$ and porosity $\phi$ for each site I use the average Sauter diameter $\left\langle d_{S}\right\rangle$ (as described in Sec. 3.3.4). Direct measurements of the permeability of soil collected at the field sites was not feasible for a large amount of samples, since measurements took several days up to a week, due to the very low permeabilities. Nevertheless, to confirm the validity of the calculated values, I measured the permeability of a soil-mixture from samples from a depth of $z=2-50 \mathrm{~cm}$ from site T32-1-L1 (2), following the protocol detailed by [135]. I recorded a water column drop of $17.7 \mathrm{~cm}$ over the course of 65 hours, which corresponds to a permeability of $\kappa \approx 5 \cdot 10^{-14} \mathrm{~m}^{2}$. This is about $50 \%$ smaller but still within the error range of the permeability of $\kappa=(1.34 \pm 1.04) \cdot 10^{-13} \mathrm{~m}^{2}$ calculated using the Sauter diameter.

I also calculate the porosity $\phi$ following Eq. (3.17) using the average Sauter diameter. The average Sauter diameter $\left\langle d_{S}\right\rangle$ and standard deviation $\sigma_{d_{S}}$, as well as permeability and porosity for each site, are listed in Tab. 4.3. Average Sauter diameters for almost all field sites at Owens Lake lie in the range of $\left\langle d_{S}\right\rangle=10-50 \mu \mathrm{m}$, owing to the significant clay content of the samples. Sauter diameters calculated for sites located within the same management cell show similar values, which serves as a consistency check for the method. Sauter diameters for Badwater Basin are slightly higher, with an average of $\left\langle d_{S}\right\rangle=63 \mu \mathrm{m}$ but consistent amongst each other (i.e. within each other's standard deviation). Standard deviations are generally rather large: on average $\sigma_{d_{S}}=0.45 \cdot\left\langle d_{S}\right\rangle$. This is due to the significantly different grain size distributions at different depths that enter the average. 


\section{Salinity gradient}

In salt pans, $\mathrm{NaCl}$ is not the only salt present in the brine, and solute densities can be higher than the density of a pure saturated $\mathrm{NaCl}$ solution. To arrive at accurate measurements of the maximum and background densities, I extracted pore water as described in Sec. 3.3.1 from the sites at Owens Lake. I extracted samples both from water right below the salt crust as well as at a depth greater than $1 \mathrm{~m}$. Results are shown in Tab. 4.2. I find a density gradient of $\Delta \rho=\left\langle\rho_{S}\right\rangle-\left\langle\rho_{b}\right\rangle=0.210 \pm 0.011$ $\mathrm{g} / \mathrm{cm}^{3}$. These findings are consistent with measurements by [44], who reported densities of $\rho(100 \mathrm{~cm})=1.041 \mathrm{~g} / \mathrm{cm}^{3}$ and $\rho(10 \mathrm{~cm})=1.288 \mathrm{~g} / \mathrm{cm}^{3}$. An additional measurement at $\rho(230 \mathrm{~cm})=1.033 \mathrm{~g} / \mathrm{m}^{3}$ suggests that density does not decrease much further at larger depths.

I was not able to extract pore water from the sites at Badwater Basin or Bristol Playa, since these sites were much drier. I will use the density gradient measured at Owens Lake as approximation for the density gradient at these sites in the following analysis.

\begin{tabular}{|c|c|}
\hline site & density $\left[\mathrm{g} / \mathrm{cm}^{3}\right.$ \\
\hline \multicolumn{2}{|c|}{ background density } \\
\hline T32-1-L1 (1) & $1.050 \pm 0.004$ \\
\hline T32-1-L1 (2) & $1.051 \pm 0.001$ \\
\hline average $\rho_{b}$ & $1.050 \pm 0.003$ \\
\hline \multicolumn{2}{|c|}{ maximum density } \\
\hline T10-3 & $1.250 \pm 0.003$ \\
\hline $\mathrm{T} 2-5(3)$ & $1.244 \pm 0.004$ \\
\hline T27-A (3) & $1.260 \pm 0.003$ \\
\hline T16 & $1.260 \pm 0.004$ \\
\hline average $\rho_{S}$ & $1.255 \pm 0.008$ \\
\hline
\end{tabular}

TABLE 4.2: Background and maximum densities measured from pore water extracted at the field sites at Owens Lake and measured using a vibrating-tube densimeter.

\section{Field Rayleigh numbers}

Using the permeability, porosity, density gradient and evaporation rate determined for each field site, I calculate the Rayleigh number following Eq. (4.8) for each site. Rayleigh numbers are listed in Tab. 4.3 and fall in the range of $\mathrm{Ra}=9-2941$. The median Rayleigh number for the sites at Owens Lake is $\mathrm{Ra}=237$ whereas for Badwater Basin it significantly higher, with $\mathrm{Ra}=1650$. Errors for $\mathrm{Ra}$ are large because standard errors for $d_{S}$ are substantial. This can be attributed to the presence of a wide range of soil components ranging from clay to medium sand.

All sites except for site T10-3 have a Ra larger than $\mathrm{Ra}_{c}=14.34$ and even for this one site, $\mathrm{Ra}$ is larger than but comparable to the $\mathrm{Ra}_{c}=6.95$ corresponding to the critical Rayleigh number for the onset of instability in the "salt lake" boundary condition. Based on these findings I can conclude that the stratified saline water is 
prone to become unstable with respect to salinity perturbations and is expected to express convective dynamics.

\begin{tabular}{l|c|c|c|c} 
Site & $d_{s}[\mu \mathrm{m}]$ & $\kappa \cdot 10^{-13}\left[\mathrm{~m}^{2}\right]$ & $\phi \pm 10^{-2}$ & $\mathrm{Ra}$ \\
\hline Badwater Basin (1) & $59 \pm 15$ & $8.13 \pm 4.05$ & 0.33 & $1398 \pm 834$ \\
Badwater Basin (2) & $66 \pm 12$ & $9.47 \pm 3.65$ & 0.33 & $1650 \pm 835$ \\
Badwater Basin (3) & $67 \pm 9$ & $9.76 \pm 2.80$ & 0.33 & $1704 \pm 743$ \\
Badwater Basin (4) & $72 \pm 3$ & $10.58 \pm 2.29$ & 0.33 & $1862 \pm 733$ \\
Badwater Basin (5) & $50 \pm 15$ & $6.50 \pm 3.98$ & 0.34 & $1102 \pm 765$ \\
\hline Bristol Playa & $46 \pm 17$ & $5.72 \pm 5.13$ & 0.34 & $746 \pm 887$ \\
\hline T10-3 & $4.3 \pm 0.6$ & $0.07 \pm 0.03$ & 0.37 & $9 \pm 4$ \\
T16 & $6.8 \pm 1.6$ & $0.18 \pm 0.09$ & 0.36 & $22 \pm 12$ \\
T2-4 & $29 \pm 14$ & $2.67 \pm 1.83$ & 0.35 & $339 \pm 247$ \\
T2-5 (1) & $24 \pm 4$ & $1.86 \pm 0.65$ & 0.35 & $233 \pm 102$ \\
T2-5 (2) & $20 \pm 3$ & $1.31 \pm 0.38$ & 0.36 & $163 \pm 63$ \\
T2-5 (3) & $18 \pm 3$ & $1.11 \pm 0.28$ & 0.36 & $138 \pm 50$ \\
T25-3 (1) & $11 \pm 2$ & $0.34 \pm 0.15$ & 0.36 & $55 \pm 23$ \\
T25-3 (2) & $24 \pm 4$ & $1.88 \pm 0.77$ & 0.35 & $237 \pm 114$ \\
T27-A (1) & $31 \pm 8$ & $2.90 \pm 1.52$ & 0.35 & $368 \pm 215$ \\
T27-A (2) & $33 \pm 4$ & $3.27 \pm 0.30$ & 0.35 & $417 \pm 114$ \\
T27-A (3) & $22 \pm 3$ & $1.66 \pm 0.46$ & 0.36 & $208 \pm 79$ \\
T27S (*) & $21 \pm 6$ & $1.53 \pm 0.81$ & 0.36 & $191 \pm 113$ \\
T29-3 (1) & $138 \pm 23$ & $18.92 \pm 9.10$ & 0.29 & $2941 \pm 1604$ \\
T29-3 (2) & $116 \pm 22$ & $17.26 \pm 15.97$ & 0.30 & $2566 \pm 2464$ \\
T32-1-L1 (1) $(*)$ & $36 \pm 12$ & $3.77 \pm 3.01$ & 0.35 & $482 \pm 405$ \\
T32-1-L1 (2) (*) & $20 \pm 4$ & $1.34 \pm 0.44$ & 0.36 & $166 \pm 69$ \\
T32-1-L1 (3) $(*)$ & $37 \pm 10$ & $4.04 \pm 1.96$ & 0.35 & $519 \pm 285$ \\
T36-3 (1) & $46 \pm 4$ & $5.64 \pm 1.34$ & 0.34 & $735 \pm 257$ \\
T36-3 (2) & $35 \pm 8$ & $3.73 \pm 1.12$ & 0.35 & $477 \pm 189$ \\
T36-3 (3) & $75 \pm 25$ & $11.18 \pm 12.48$ & 0.33 & $1535 \pm 1758$ \\
T8-W & $8.7 \pm 2.6$ & $0.29 \pm 0.18$ & 0.36 & $35 \pm 23$ \\
\hline
\end{tabular}

TABLE 4.3: Sauter diameter $d_{S}$, permeability $\kappa$, porosity $\phi$ and Rayleigh number Ra measured or calculated for each of the field sites at Owens Lake, Badwater Basin and Bristol Playa. Samples stemming from a trench are indicated as $(*)$.

\subsubsection{Instability under laboratory conditions}

I conducted a series of experiments to investigate the convective flows in settings similar to the ones found in salt pans in nature. The experimental methodology is described in Sec. 3.2. As with the field sites described above, the first step is to determine whether experimental conditions give a Rayleigh number above $\mathrm{Ra}_{c}$, and should therefore be subject to a convective instability.

To calculate the Rayleigh number following Eq. (4.8), I consider viscosity to be constant. Salinity gradients in the experiments were also kept at a constant density difference of $\Delta \rho=1.162 \mathrm{~g} / \mathrm{cm}^{3}$. Evaporation rate in the experiments conducted for the investigation of the onset of convection was $E \approx 3.6 \cdot 10^{-7} \mathrm{~m} / \mathrm{s}$. I varied the grain size of the porous medium in the Hele-Shaw cell to vary $\kappa$ and therefore scan for the 
onset of convection at different Ra. I determined $d_{S}$ and calculated $\phi$ for each grain size used in the experiment as described in Sec. 3.2.2 and Sec. 3.2.2. Permeability was directly measured, as described in Sec. 3.2.2.

All values and calculated Rayleigh numbers are listed in Tab. 4.4. All Rayleigh numbers except for the setup with a grain size distribution in the range of $d=0-20 \mu \mathrm{m}$ are above $\mathrm{Ra}_{c}$. This is consistent with the observation of the convective dynamics in the experiments: all setups showed convective dynamics, except for the experiment with the porous medium of the smallest grain size distribution.

\begin{tabular}{c|c|c|c|c|c} 
grain size & $d_{S}[\mu \mathrm{m}]$ & $\kappa\left[\mathrm{m}^{2}\right]$ & $\phi \pm 10^{-2}$ & Ra & convection \\
\hline $0-20$ & $1.9 \pm 0.2$ & $1.5 \pm 0.3 \cdot 10^{-15}$ & 0.37 & $0.02 \pm 0.01$ & no \\
$70-110$ & $86 \pm 9$ & $0.77 \pm 0.13 \cdot 10^{-11}$ & 0.32 & $107 \pm 18$ & yes \\
$90-150$ & $123 \pm 12$ & $1.44 \pm 0.1 \cdot 10^{-11}$ & 0.30 & $214 \pm 15$ & yes \\
$100-200$ & $150 \pm 15$ & $1.67 \pm 0.12 \cdot 10^{-11}$ & 0.28 & $263 \pm 18$ & yes \\
$150-250$ & $214 \pm 20$ & $4.7 \pm 0.2 \cdot 10^{-11}$ & 0.24 & $857 \pm 34$ & yes \\
$200-300$ & $264 \pm 25$ & $8.27 \pm 0.3 \cdot 10^{-11}$ & 0.21 & $1725 \pm 57$ & yes \\
\hline
\end{tabular}

TABLE 4.4: Sauter diameter $d_{S}$, permeability $\kappa$, porosity $\phi$ Rayleigh number Ra for each of the experimental setups.

\subsubsection{Summary}

I found Rayleigh numbers above the critical value for the onset of buoyancy-driven porous-media convection at 26 out of 27 field sites. For the experimental setups, I found that all setups except the one with the smallest grain size of $0-20 \mu \mathrm{m}$, corresponding to clay, have $\mathrm{Ra}>\mathrm{Ra}_{c}$. I was able to directly observe convective dynamics for all setups, except the setup with $\mathrm{Ra}<\mathrm{Ra}_{c}$. This is consistent with results from [41], who conducted a series of experiments with Rayleigh numbers between $\mathrm{Ra}=0$ and $\mathrm{Ra}=22.8$ for the "salt lake" boundary condition and found the onset of convection between $\mathrm{Ra}=5.8$ and $\mathrm{Ra}=8.9$, as predicted by the linear stability analysis for this boundary condition (see Tab. 4.1).

I conclude that both in the laboratory as well as in the field, the system are unstable to salinity perturbations. I therefore expect the existence of plumes of higher salinity to be detectable both in the experiment as well as in the field. In the following sections I will show direct hydrological evidence for the existence of such plumes.

\subsection{Density stratification}

In this section I will investigate in numerical simulations, how salinity gradients behave at different times and Rayleigh numbers. I will then use the results from the simulations to interpret the salinity gradients found in the experiment and the field sites. 
Simulations conducted for this investigation were implemented as two-dimensional systems scaled by the Rayleigh number, as described in Sec. 3.1.

\subsubsection{System age}

The dynamics in the simulated systems occur on vastly different timescales, depending on system Rayleigh number. I qualitatively illustrated this in three visualizations of simulations at different Ra and $\tau$, shown in Fig. 3.2. To account for the increased vigor of the high-Ra convection and to quantitatively compare simulations at different times and Rayleigh numbers to each other, I introduce the time $\tau_{S}$ as relative age of the system, as described in Sec. 3.1.8. $\tau_{S}$ is the time it takes until the salinity reaches a value of 0.5 or greater at a depth of $Z=-1$ for the first time. This is comparable to a relative speed of the dynamics. I find a power law dependence of $\tau_{S}$ on $\mathrm{Ra}-\mathrm{Ra}_{c}$ of the form

$$
\tau_{S}\left(\mathrm{Ra}-\mathrm{Ra}_{c}\right)=(62.3 \pm 4.1)\left(\mathrm{Ra}-\mathrm{Ra}_{c}\right)^{-1.28 \pm 0.04},
$$

as plotted in Fig. 4.3. Values are averages and standard deviations of ensembles of 7-9 simulation runs with the same simulation parameters but different seeds for the noise in the salinity distribution initial condition. Recovery of a power law in $\tau_{S}$ confirms self-similarity of the system when the system time is scaled with $\tau_{S}$ and critical slowing down [165], as the system approaches the critical point. In the following, I use $\tau_{S}$ to compare systems at different Ra but similar relative ages.

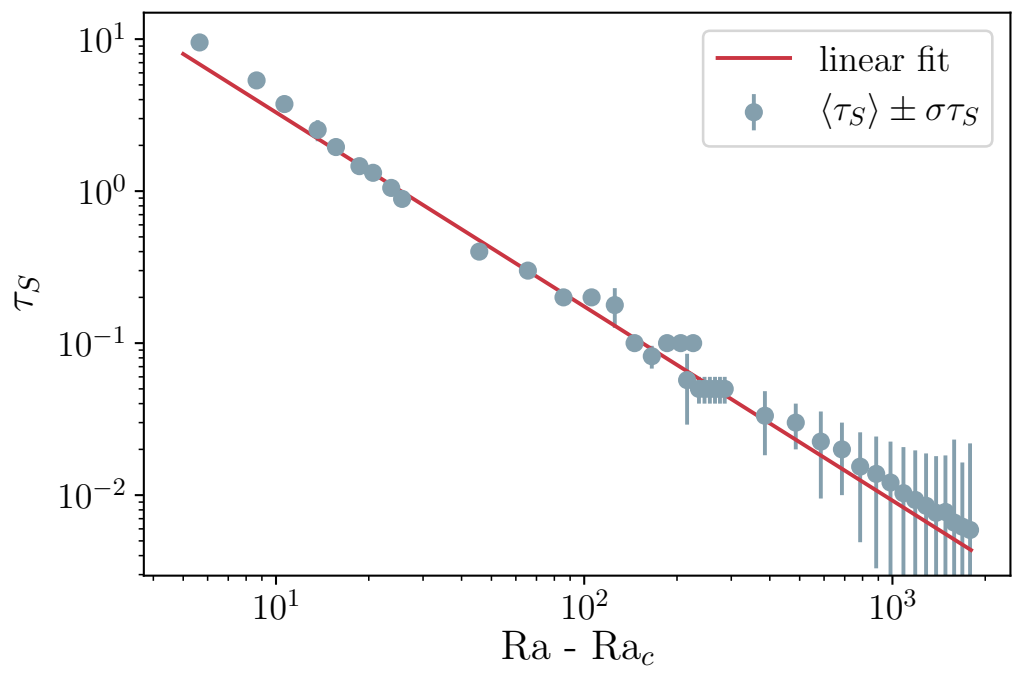

FIGURE 4.3: Double logarithmic plot of the time $\tau_{S}$ when $S(Z=1) \geq 0.5$ for the first time, over $\mathrm{Ra}-\mathrm{Ra}_{c}$ for $\mathrm{Ra}=20-2000$. Data points (gray) are averages and standard deviations over ensembles with 5-10 runs. Red line: fit $\tau_{S}\left(\mathrm{Ra}-\mathrm{Ra}_{c}\right)=(62.3 \pm 4.1)\left(\mathrm{Ra}-\mathrm{Ra}_{c}\right)^{-1.28 \pm 0.04}$ to ensemble averages weighted by the standard deviations. 


\subsubsection{Properties of the salinity gradient}

In the steady state the salinity distribution in the Z-direction given in Eq. (2.24) follows an exponential decay with depth, characterized by the length scale $L=1$. Since in a system which exhibits convection I do not expect the steady state salinity distribution to be preserved, I expect to find a length scale $L^{\prime} \leq L$. To find the length scale $L^{\prime}$, I fit a function of the form

$$
f(z)=C_{0}+\left(C_{1}-C_{0}\right) \cdot e^{z / L^{\prime}}
$$

to the salinity distributions measured in the simulations, experiment and field. Here, $C_{0}$ corresponds to a non-zero background salinity the salinity will decay to, whereas $C_{1}=1$ is the maximum salinity.

In Fig. 4.4 (a) I show, how the salinity distribution $S(Z)$ for simulations at $\mathrm{Ra}=10^{3}$ develops with relative system age $\tau_{S}$, as defined in Sec. 4.3.1. In Fig. 4.4 (b) I show the length scale $L^{\prime}$ as well as the background salinity $C_{0}$ from fits of Eq. (4.10) to the salinity distributions between $Z=0$ and $Z=-0.2$. Fits start at $\tau=2 \tau_{S}$, since before that, the salinity distribution is in a transient state between the initial condition and a much steeper salinity decay, and is not well described by Eq. (4.10), therefore the fitting routine does not converge. Measurements are averages and standard deviations of ensembles of 8 simulation runs.

The length scale $L^{\prime}=0.033 \pm 0.001$ of the salinity gradient is much smaller than the length scale of $L^{\prime}=1$ corresponding to the steady state solution. Therefore the decay of the salinity distribution is much steeper, since convective mixing dominates and quickly strips away excess salinity from the top boundary. The background salinity approaches $C_{0} \rightarrow 0.27 \pm 0.01$.
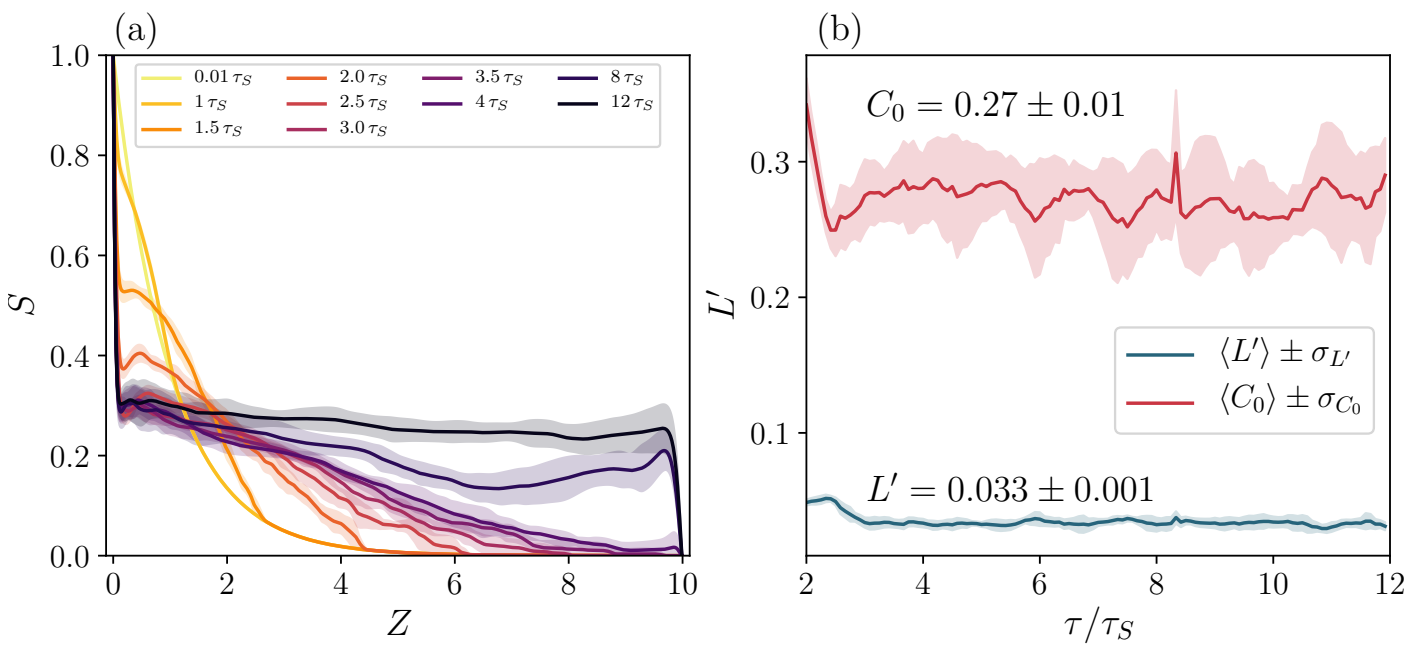

FIGURE 4.4: (a) Salinity distribution $S(Z)$ for system ages $\tau_{S}=0.1$ to $\tau_{S}=10$. (b) Length scales $L^{\prime}$ and background salinities $C_{0}$ from fits to the salinity distributions between $Z=0$ and $Z=-0.8$. Simulations were conducted at $\mathrm{Ra}=10^{3}$, measurements are ensemble averages and standard deviations over 8 simulation runs. 
In Fig. 4.5 (a) I show salinity distributions at a fixed relative system age of $\tau=5 \tau_{\text {S }}$ for a selection of Rayleigh numbers $\mathrm{Ra} \in\{23,100,200,500\}$. In Fig. 4.5 (b), the length scale $L^{\prime}$ and background salinity $C_{0}$ for a range of simulations at Rayleigh numbers between $\mathrm{Ra}=23$ and $\mathrm{Ra}=1.8 \cdot 10^{3}$ are shown. The length scale was determined from fits to the salinity distribution in a shrinking window for increasing Rayleigh numbers. Windows ranged between $Z=-4$ to $Z=0$ for $\mathrm{Ra}=23$ to $Z=-0.5$ to $Z=0$ for $\mathrm{Ra}=1.8 \cdot 10^{2}$. The background salinity $C_{0}$ quickly rises and approaches $C_{0} \rightarrow 0.29 \pm 0.2$, similar to the background salinity in Fig. 4.4 (b). The length scale $L^{\prime}$ drops quickly and seems to approach zero as Rayleigh numbers increase.
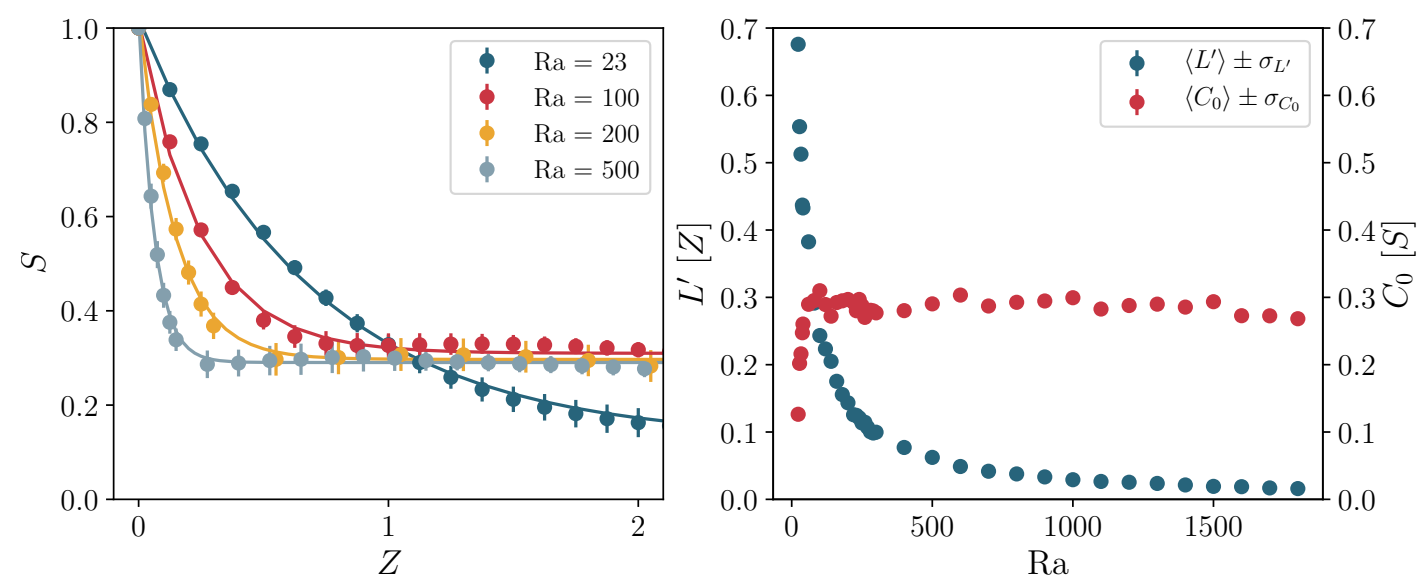

FIGURE 4.5: (a) Salinity distribution $S(Z)$ for four different Ra at $\tau=5 \tau_{S}$. (b) Length scale $L^{\prime}$ and background salinity $C_{0}$ for a range of different Ra. Values are ensemble averages and standard deviations over 7-9 simulation runs.

\subsubsection{Salinity gradients in the experiment}

To measure the salinity gradient in the analogue experiments, I dissected one HeleShaw setup (see Sec. 3.2.1 for details) and took samples of the porous medium saturated with saline water along several vertical columns. The sampling process as well as sampling positions with respect to the setup dimensions are described in more detail in Sec. 4.4.1 and Fig. 4.9. The experiment at the time of sampling had a Rayleigh number of $\mathrm{Ra} \approx 1000$.

I determined the salt concentration following the protocol described in Sec. 3.2.4 and calculated the salinity following Eq. (2.8). The evaporation rate of the experimental setup before it was dissected was approximately $E \approx 9 \cdot 10^{-8} \mathrm{~m} / \mathrm{s}$. This leads to a natural length scale of $L=D / E \approx 1 \mathrm{~cm}$. Since the Rayleigh number of the experiment was of the order $\mathcal{O}\left(10^{3}\right)$, judging from the simulations shown in Fig. 4.4, I expect the gradient relevant for the instability to build up in the topmost $0.5-1 \mathrm{~cm}$ of the cell. Since I only sampled with a resolution of $\Delta z=2 \mathrm{~cm}$, I do not have enough data points to fit an exponential decay to the data and get a reliable result for $L^{\prime}$. I nevertheless show the results of the salt concentration measurement in the analogue experiment in Fig. 4.6. The values are averages over 9 samples in the $X$-direction for every depth $Z \in\{2,4, \ldots, 16\}$. It is clearly visible that there is a 
salinity gradient from top to bottom, even though the resolution and uncertainty of the measurements do not allow for its quantification.

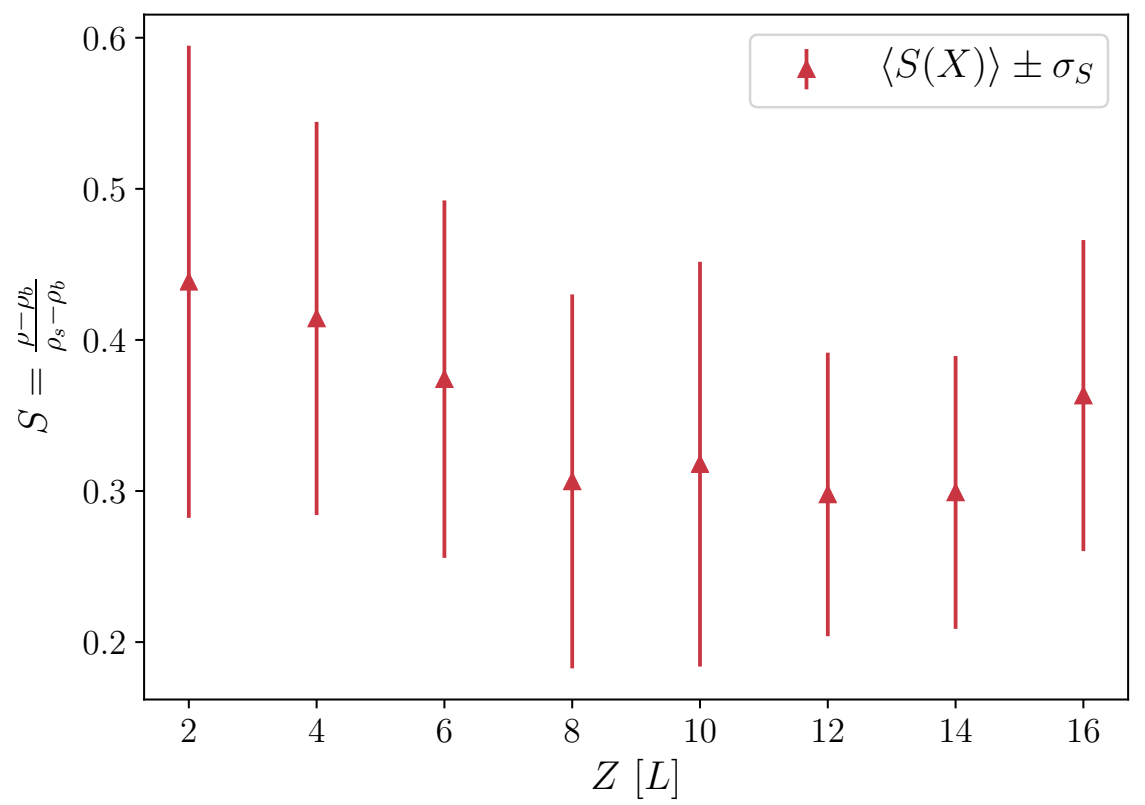

FIGURE 4.6: Salinity measurements from a Hele-Shaw setup. Values are averages and standard deviations from 9 samples taken at the same depth each.

\subsubsection{Salinity gradients in the field}

\section{Effective diffusivity in the field}

So far, my model uses a molecular diffusion coefficient of $D=1.61 \cdot 10^{-9} \mathrm{~m}^{2} / \mathrm{s}$. This corresponds to the diffusion of pure $\mathrm{NaCl}$ in infinitely small quantities at room temperature in water. Moreover, since I model a flux through a porous medium, I also neglected the tortuosity of the porous matrix. In the following I will revisit these simplifications to arrive at a more accurate estimation of the molecular diffusion coefficient.

In the field, I encountered a mixture of several salts, most notably sodium chloride and sodium sulfate. Details of the quantitative analysis of the salt species using both quantitative XRD as well as spectrometry are given and discussed in Appendix B. Therefore the diffusion process is, according to [166], determined by the main diffusion coefficient of individual species and the cross-diffusion coefficients between the species. The mass ratio of $\mathrm{NaCl}$ to sodium sulfate in the pore water is roughly $5 / 3$ (see Tab. B.2). At maximum salinity or $\rho_{b} \approx 1.25 \mathrm{~g} / \mathrm{cm}^{3}$, this corresponds to $14 \mathrm{~mol} / 1 \mathrm{NaCl}$ and $3 \mathrm{~mol} / 1 \mathrm{Na}_{2} \mathrm{SO}_{4}$, or a molar ratio of 0.82 . Diffusion coefficients of ternary mixtures of both salts in water at $25^{\circ} \mathrm{C}$ were measured by [167]. For a mixture of $1.35 \mathrm{~mol} / \mathrm{kg} \mathrm{NaCl}$ and $0.15 \mathrm{~mol} / \mathrm{kg} \mathrm{Na}_{2} \mathrm{SO}_{4}$ they find $D_{\mathrm{NaCl}}=1.5 \cdot 10^{-9} \mathrm{~m}^{2} / \mathrm{s}$ and $D_{\mathrm{Na}_{2} \mathrm{SO}_{4}}=0.74 \cdot 10^{-9} \mathrm{~m}^{2} / \mathrm{s}$ for the two main components $D_{11}$ and $D_{22}$ of the diffusion tensor. The the mole ratio of $\mathrm{NaCl}$ to $\mathrm{Na}_{2} \mathrm{SO}_{4}$ of 
0.9 used by [167] for their experiments is quite similar to the ratio of the two salts found in the field.

Diffusion coefficients for a single ion depend on its own concentration as well as the type and concentration of all other ions in the solution and the temperature of the fluid. Given these multiple dependencies, it is challenging to accurately predict the relevant diffusion coefficient for a system as complex as the brines I found in the field. To nevertheless refine my approximation of the diffusion coefficient, I assume that the measured concentration gradient will most likely be the result of a superposition of the diffusion coefficients of sodium chloride and sodium sulfate. Since I use a scalar diffusion coefficient for my model rather than a full diffusion tensor, I resort to the rather simplistic approach of calculating the average weighted of the two main components weighted by their mole ratio. This yields $D=1.37 \cdot 10^{-9} \mathrm{~m}^{2} / \mathrm{s}$. I note that in this calculation I still neglect the mutual diffusion cross-term diffusion coefficients $D_{21}$ and $D_{12}$ for diffusion of sodium sulfate due to gradients of sodium chloride and vice versa. Nevertheless this seems warranted because, since both are rather small $\left(D_{21} \rightarrow 0\right.$ for high ratios of $\mathrm{NaCl}$ in the mixture and $D_{12} \approx 0.2 \mathrm{~m}^{2} / \mathrm{s}$ according to [167]). I also note that the estimate of the evaporation rate is still a large source of uncertainty in this calculation that can not easily be reduced.

While I can safely neglect tortuosity $\theta$ for the advective transport of fluid, since Darcy's equation only considers the superficial velocity i.e. the Darcy velocity, I have to take it into account for diffusive processes, since they occur on the molecular scale. I calculate the tortuosity from the porosity based on the modified Weissbergrelation $[168,169]$

$$
\theta^{2}=1-\ln \left(\phi^{2}\right)
$$

For porosities of $\phi \approx 0.36$ this yields values of $\theta^{2} \approx 2$. This means that ions have to travel a path about twice as long in a porous medium to cover the same distance as outside it. According to [170], the modified diffusion coefficient $D^{*}$ is then calculated as $D^{*}=D / \theta^{2} \approx 0.63 \cdot 10^{-9} \mathrm{~m} / \mathrm{s}$.

Using the modified diffusion coefficient, and an evaporation rate of $E=4.6$. $10^{-9} \mathrm{~m} / \mathrm{s}$ (see Sec. 3.3.4), the natural length scale of the system predicted as the ratio of the diffusion coefficient and the evaporation rate is $L \approx 27 \mathrm{~cm}$ for Owens Lake. At Badwater Basin, the evaporation rate is estimated to be a little bit lower with $E=2.1 \cdot 10^{-9} \mathrm{~m} / \mathrm{s}$ and therefore $L \approx 60 \mathrm{~cm}$.

\section{Salinity gradient length scale}

Similar to the approach described in Sec. 4.3.3, I took samples from horizontal crosssections below polygons, as described in Sec. 3.3.1 and measured the salinity. At many sites, salinity measurements were not accurate and reliable enough to draw reliable conclusions about the salinity gradient, because of noise introduced by the 


\begin{tabular}{l|ccccc} 
site & $L^{\prime}$ & $C_{0}$ & $\rho_{s}\left[\mathrm{~g} / \mathrm{cm}^{3}\right]$ & $\mathrm{Ra}$ & $N_{X}$ \\
\hline T27-S & $0.95 \pm 0.37$ & $0.44 \pm 0.06$ & 1.23 & 191 & 17 \\
T32-1-L1 (2) & $1.38 \pm 0.13$ & $-0.01 \pm 0.03$ & 1.30 & 166 & 16 \\
T32-1-L1 (3) & $1.24 \pm 0.10$ & $0.01 \pm 0.03$ & 1.22 & 519 & 18 \\
T32-1-L1 (1) & $1.06 \pm 0.14$ & $0.21 \pm 0.03$ & 1.31 & 482 & 7 \\
T16 & $1.40 \pm 0.14$ & $0.40 \pm 0.02$ & 1.34 & 22 & 11 \\
T27-A (1) & $2.35 \pm 1.19$ & $0.39 \pm 0.15$ & 1.66 & 368 & 5 \\
T25-3 (2) & $0.53 \pm 0.04$ & $0.10 \pm 0.01$ & 1.72 & 237 & 13 \\
T29-3 (1) & $1.04 \pm 0.46$ & $0.12 \pm 0.26$ & 1.35 & 2941 & 7 \\
T29-3 (2) & $0.67 \pm 0.19$ & $0.29 \pm 0.06$ & 1.64 & 2566 & 5 \\
\hline
\end{tabular}

TABLE 4.5: Length scale and background salinity determined from fits to the measured salinity gradient for nine field sites. Ra is given for reference (see Tab 4.3 for error ranges) and $N_{X}$ denotes the number of samples for each depth $Z$.

sampling methodology (see Sec. 4.4.2 for a detailed discussion of field results reliability). As a result, I was able to calculate reliable salinity gradients from all trench sites (sites T32-1-L1 (1), (2) and (3) as well as site T27-S) and five additional sites where I used the Dutch Gauge corer (T27-A (1) and (2), T29-3 (1) and (2) and T16).

Similar to the Hele-Shaw experiment, to determine the salinity gradient $S(Z)$ I first averaged over the $X$-direction to calculate the density at a given depth $Z$. To calculate the salinity $S$ following Eq. (2.8), I used the background density $\rho_{b}$ measured at sites T32-1-L1 (1) and (2) (see Tab. 4.2). As maximum density $\rho_{s}$ I used the maximum density measured at each site. Averaged salinity measurements and standard deviations (red dots) and fits (blue line) for nine field sites are shown in Fig. 4.7.

For many sites, the maximum salinity was two to four times larger than the maximum salinity $\rho_{s}$ listed in Tab. 4.2. This can be explained by sub-efflorescence in samples that were taken close to the crust. The salt crystals in the samples dissolved during the salt concentration measurement protocol in the laboratory (see Sec. 3.2.4 for details), and therefore effectively increased $\rho_{s}$ to higher values. I nevertheless chose to use $\max (\rho)$ measured at each site as $\rho_{s}$, to keep the salinity normalized to $S=[0,1]$. I also excluded the samples taken at $z=2 \mathrm{~cm}$ and $z=10 \mathrm{~cm}$ from the fit at sites T32-1-L1 (3) and T27-S, as well as samples from $z=2 \mathrm{~cm}$ from sites T16 and T29-3 (1), since they by far exceeded the maximum solubility of $\mathrm{NaCl}$ and $\mathrm{Na}_{2} \mathrm{SO}_{4}$ in water, which seemed unphysical. After excluding the unphysical values, maximum densities at the field sites ranged from $\rho_{s}=1.23 \mathrm{~g} / \mathrm{cm}^{3}$ to $\rho_{s}=1.72 \mathrm{~g} / \mathrm{cm}^{3}$.

The length scale $L^{\prime}$ and background salinity $C_{0}$ were determined from fits of a function of the form given in Eq. (4.10) to the salinity distribution. Maximum densities, results from the fits as well as the number of samples $N_{X}$ entering the averaging at each depth $Z$ are listed in Tab. 4.5. Errors are standard deviations calculated from the covariance matrix of the fits.

Length scales $L^{\prime}$ range between $L^{\prime} \approx 0.5$ and $L^{\prime} \approx 2.4$. Given the results from the numerical simulations shown in Fig. 4.5, length scales larger than $L^{\prime}=1$ do not seem physical. Even for the four trench sites T27-S and T32-1-L1 (1), (2) and (3), from which I expect the most accurate salt concentration measurements (see Sec. 4.4.2 for 

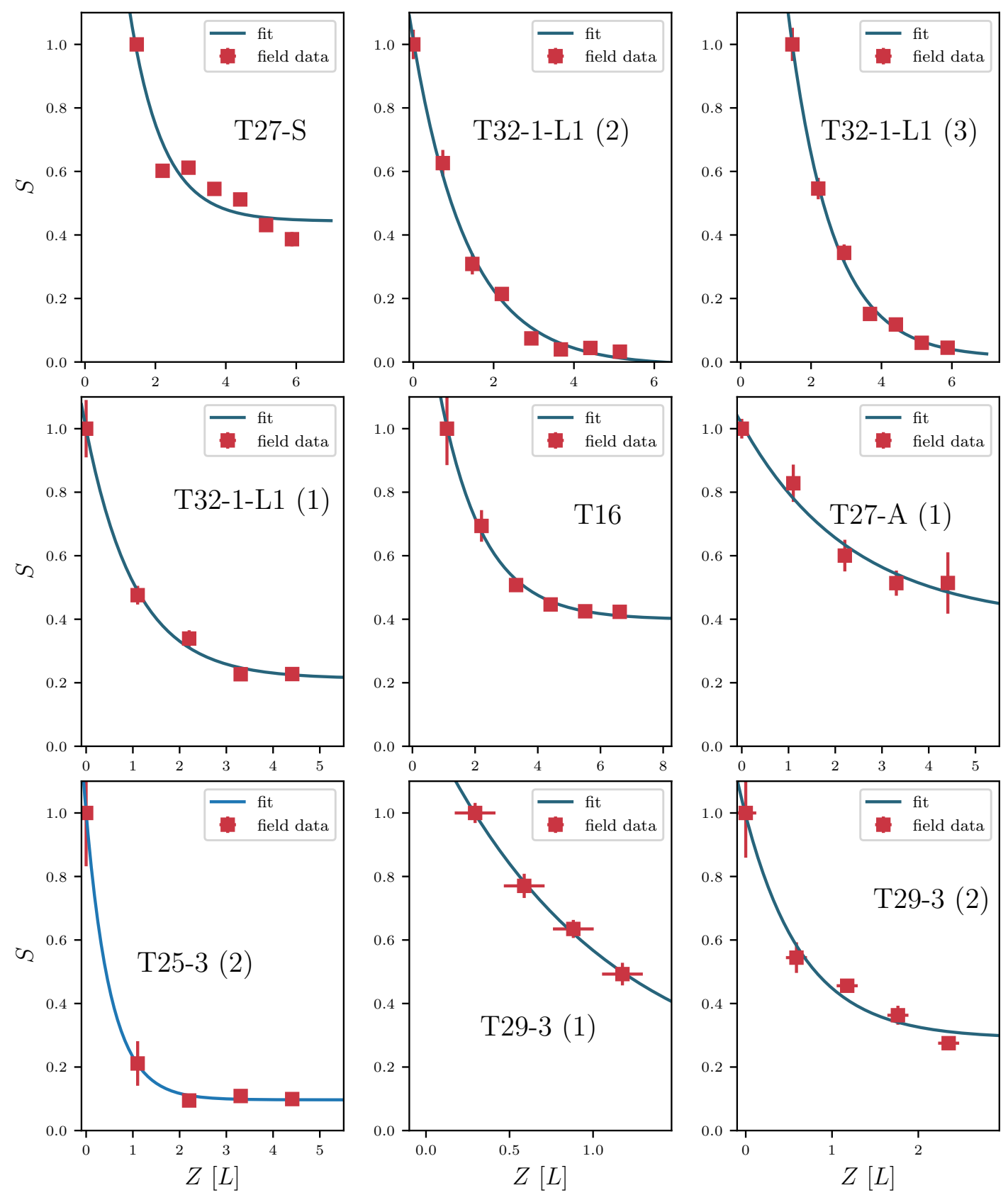

FIGURE 4.7: Salinity (red dots) measured in the field and fits to determine $L^{\prime}$ and $C_{0}$ for the field sites. Values for $L^{\prime}$ and $C_{0}$ are listed in Tab. 4.5. Field measurements are averages over all samples taken at a specific depth. Error bars are standard deviations.

a discussion about uncertainties in the field measurements), two out of four values are significantly larger than one. Additionally, there is no clear trend of decreasing $L^{\prime}$ with increasing $\mathrm{Ra}$, as suggested by the results from the simulations. Background salinities $C_{0}$ appear similarly scattered. These results can probably be explained by both the high uncertainty of the measured Rayleigh numbers (see Tab. 4.3 for error ranges) as well as the fact that the topmost samples are most prone to contamination by salt crystals from the surface salt crust. Additionally, since at Owens Lake the natural length scale is estimated to be $L \approx 30 \mathrm{~cm}$, I expect most of the relevant 
salinity gradient to lie within that range. Once again, the vertical resolution of the samples of $\Delta z=10 \mathrm{~cm}$ does not allow for a reliable quantification of the gradient.

I conclude that while I can not quantify the properties of the density gradients in the field, they do exist and, to some extent, follow my predictions about their behavior i.e. seem to follow an exponential decay at most sites. While a quantification of the behavior of the gradient in the field setting needs more research and a higher sampling resolution, the existence of vertical salinity gradients is a strong predictor for the existence of buoyancy driven convection in the system.

\subsubsection{Summary}

I introduced the time $\tau_{S}$, which corresponds to the time a simulation takes until the salinity at $Z=1$ surpasses a value of 0.5 for the first time. This characteristic time follows a power-law with $\mathrm{Ra}-\mathrm{Ra}_{c}$. I subsequently used multiples of $\tau_{S}$ to define the age of a system and quantitatively compare systems with different Rayleigh numbers.

Based on the steady state salinity distribution calculated in Sec. 2.6.2 I investigated the salinity gradients that emerge in my simulations of buoyancy driven porous media convection. I found that the length scale $L^{\prime}$, which characterizes the salinity decay from the top boundary downwards, depends both on relative system age $\tau_{S}$ as well as the Rayleigh number. $L^{\prime}$ drops monotonically in the investigated Rayleigh number range of $\mathrm{Ra}=23-1.8 \cdot 10^{3}$ and seems to approach zero for high Rayleigh numbers. The background salinity $C_{0}$ rises quickly and saturates at the same value of $C_{0} \approx 0.28$ for high Ra and/or high relative system ages.

I directly measured the vertical salinity gradient in one Hele-Shaw experiment. The resolution of the measurement is not high enough to reliably quantify the properties of the salinity gradient. Nevertheless, a clear gradient from the top to the bottom of the experiment is discernible.

I was able to recover the characteristic length scale of the salinity gradient from fits to salt concentration measurements in nine field sites. To arrive at a sensible natural length $L=D / E$ to scale the system, I calculated a modified diffusion coefficient, taking into account the presence of different ions in the solute as well as the tortuosity of the porous medium. Given the high error range of the Rayleigh numbers calculated for the field sites, I cannot conclude whether the characteristic length scales $L^{\prime}$ of the salinity gradients in the field sites follow the trend indicated by the numerical simulations. Nevertheless, all analyzed field sites show a pronounced decay of salinity as distance from the surface increases.

Results from the experiment and field are noisy and restrict a direct comparison to the results from the numerical simulations. Nevertheless, the existence of steep density gradients in the fluid, especially in the field setting, is a strong predictor for the existence of buoyancy driven convection in the field. 


\subsection{Convection}

In this section I present direct hydrological evidence for the existence of high salinity plumes, i.e. horizontal salinity gradients, in the Hele-Shaw experiments as well as in the field. Additionally, I show that the high salinity plumes or downwellings of the convective dynamics in the field are co-located with the patterns visible on the top. This further supports my argument that the polygonal patterns found in salt deserts are linked to underground convection.

The following arguments rely on salt concentration measurements of the samples collected both from the experiments as well as the field sites determined as described in Sec. 3.2.4. I note that all salt concentrations $C$ are given as weight ratio (wt. \%) of salts to pore fluid (i.e. water plus salts) in the following analysis.

\subsubsection{Convection in experiments}

\section{Flow dynamics}

As predicted by the linear stability analysis described in Sec. 4.2.1, all Hele-Shaw experiments with $\mathrm{Ra}>\mathrm{Ra}_{c}$ showed convective dynamics. I visualized these dynamics by injecting color into the experiments, as described in Sec. 3.2.1. The experimental setups allowed me to investigate the dynamics at system depths of $Z=-2 L$ to $Z=-75 L$ (depending on the evaporation rate), as well as large times up to $\tau / T=2 \cdot 10^{3} T$, which were not accessible in simulations, due to the computational cost of very long simulation runs.

To investigate the advection-diffusion dynamics in the experiment, I chose to keep the grain size fixed at $d=100-200 \mu \mathrm{m}$ and therefore also fix the permeability $\kappa$. To vary the Rayleigh number I varied the evaporation rate $E$ and was able to achieve evaporation rates up to $E=1.17 \cdot 10^{-6} \mathrm{~m} / \mathrm{s}$, and therefore drive the system at a speed which was about 250 times faster than the dynamics in the field. By varying the evaporation rate, I was able to access Rayleigh numbers in the range of $\mathrm{Ra}=80-2770$, which covers the whole range of Rayleigh numbers observed in the field.

I show four exemplary coloring experiments in Fig. 4.8: experiments (a) to (c) were conducted in cells with a width of $40 \mathrm{~cm}$ whereas the experiment shown in (d) was conducted in a $60 \mathrm{~cm}$ wide cell. The images illustrate the emergence of upwellings and downwellings with a clear wavelength. The evaporation rate, natural length scale, Rayleigh number, system time at measurement since the setup of the experiment (given in units of the natural time $T$ ), and measured wavenumber for the four experiments are listed in Tab. 4.6. A more detailed discussion of the scaling follows in Sec. 4.5. 

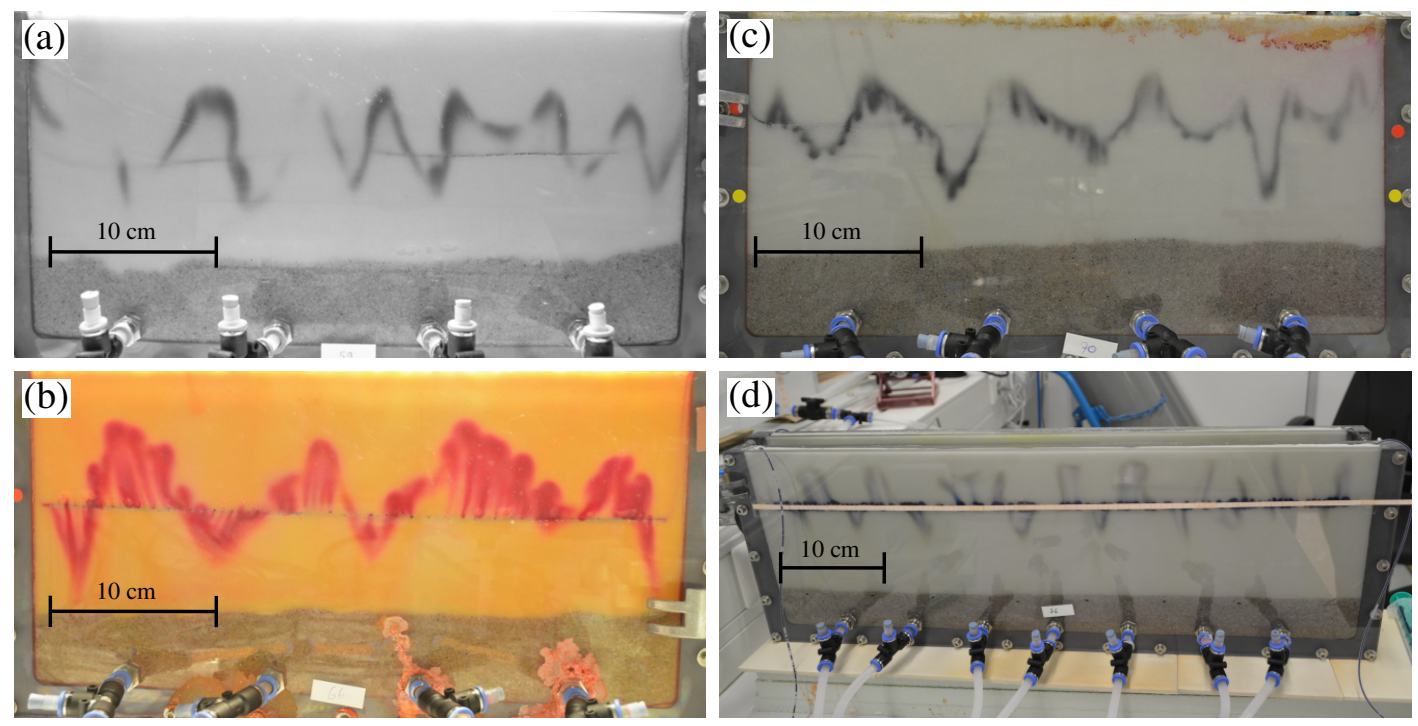

FIGURE 4.8: Images of four coloring experiments in Hele-Shaw cells visualizing the upwellings and downwellings in the cell. Grain size in the experiments was 100-200 $\mu \mathrm{m}$, Rayleigh numbers at the time of the coloring were (a) $\mathrm{Ra}=457$, (b) $\mathrm{Ra}=220$, (c) $\mathrm{Ra}=1034$ and (d) $\mathrm{Ra}=563$. Images were taken $125 \mathrm{~min}, 104 \mathrm{~min}$, $208 \mathrm{~min}$ and $120 \mathrm{~min}$ after color injection respectively.

\begin{tabular}{c|c|c|c|c}
$E \cdot 10^{-7}[\mathrm{~m} / \mathrm{s}]$ & $L[\mathrm{~mm}]$ & $a$ & $\tau / T$ & $\mathrm{Ra}$ \\
\hline $4.3 \pm 0.1$ & $3.7 \pm 0.1$ & $0.24 \pm 0.05$ & 203 & $220 \pm 14$ \\
$2.1 \pm 0.8$ & $7.7 \pm 3.0$ & $0.91 \pm 0.17$ & 6 & $457 \pm 144$ \\
$0.9 \pm 0.1$ & $17.5 \pm 2.6$ & $1.57 \pm 0.41$ & 4 & $1033 \pm 18$ \\
$1.5 \pm 0.1$ & $10.7 \pm 0.1$ & $0.89 \pm 0.11$ & 67 & $634 \pm 5$ \\
\hline
\end{tabular}

TABLE 4.6: Values for $E, L, \tau$ and Ra and measurement of the wavenumber $a$ for the four exemplary experiments shown in Fig. 4.8.

\section{Salt concentration distribution}

To determine whether the dynamics observed in the Hele-Shaw experiments were indeed driven by salinity gradients and to quantify the magnitude of the salinity signal, I measured salinity profiles directly in one of my experiments. I colored the experiment to visualize the various flows in the fluid, stopped the inflow and carefully dissected the cell to collect samples. I removed the fine sand from the cell in layers with a width of approximately $1 \mathrm{~cm}$, taking samples in every second layer. I collected samples along 5 downwellings (pink) and 4 upwellings (yellow) with a vertical resolution of about $2 \mathrm{~cm}$. The sampling process took approximately one hour. During this time, diffusive transport covers a distance of approximately $6 \cdot 10^{-6} \mathrm{~m}^{2}$ in the $x$ and $z$-direction. Advective transport given the rather low evaporation rate of $E \approx 9 \cdot 10^{-8} \mathrm{~m} / \mathrm{s}$ and the ensuing low fluid velocities in the experiments covered $0.5-1 \mathrm{~cm}$ in the $z$-direction. I therefore assume, that the snapshot of the salt concentration profile I measured is not distorted by the dynamics of the system.

The colored experiment is visualized in Fig. 4.9. The salt concentration measured at each location is indicated in the color-coded overlay where yellow indicates low 
salt concentration and purple indicates high salt concentration. It is clearly visible that regions of high salt concentration (10-14 wt.\%) are co-located with downwellings, whereas regions with background salt concentration or slightly higher of 6-8 wt.\% are co-located with upwellings. The downwellings show a salt concentration which is about two times higher than the background salt concentration of $4.8 \mathrm{wt} \%$, which is set by the salt concentration in the water reservoir feeding the experiment. The bottom most samples, which were taken in the region of coarse sand above the inlets, show a uniform salt concentration close to reservoir salt concentration. This is another indicator that the flows present in the cell are not dependent on the position of the inlets.

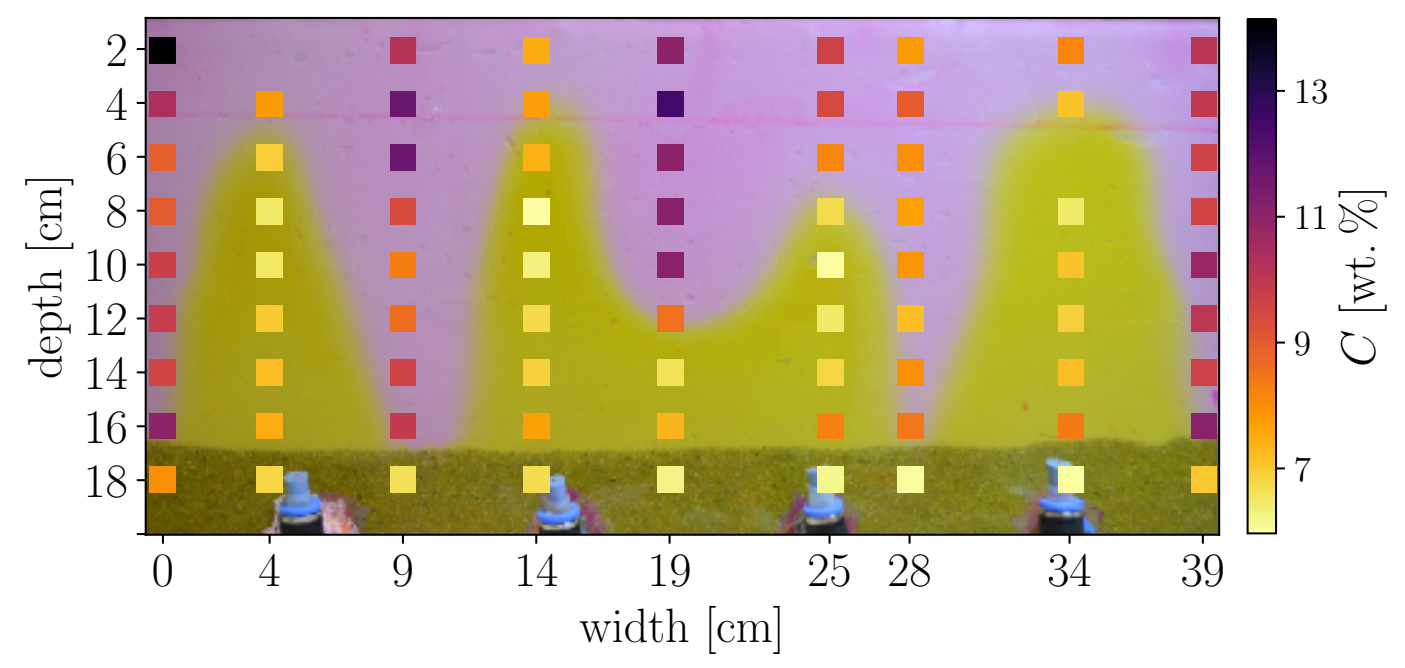

FIGURE 4.9: Direct measurement of salt concentration in a Hele-Shaw experiment. The cell was dyed with rhodamine first and later colored with fluorescein from below to the visualize flow patterns (background image). Water inflow was then stopped and the cell was dissected layer by layer, taking samples along up- and downwellings. The overlaid colormap shows salinity at each sampling location.

Based on the salinity gradient between upwelling and downwelling fluid in the experiment, I expect that the downwelling plumes in the field will have a similar salt concentration of approximately two times the field background salt concentration.

\section{$3 \mathrm{D}$ convection}

Supplementary experiments in 3D setups conducted by Birte Thiede during her work on her Bachelor's thesis showed results similar to the two-dimensional HeleShaw setups. Color was injected into the experiments at the inlets at the bottom and the porous medium was subsequently removed layer by layer to uncover the flow paths. As shown in Fig. 4.10 for two experiments. Panels (a), (b) and (c) correspond to the first experiment, panels (d), (e) and (f) to the second. Panels (a) and (d) show layers at $z=18 \mathrm{~cm}$, panels (b) and (e) show layers at $z=10 \mathrm{~cm}$ and panels (c) and (f) show layers at $z=2 \mathrm{~cm}$. For both experiments, the yellow/orange color first rose to 
the top of the experiment at the positions of the inlets (green circles) and than sank down again in plumes arranged in a circle around the inlets (blue circles). This is suggestive that the convective dynamics in three dimensions are comparable to the two-dimensional dynamics because of the low Reynolds number of the system (as stated in Sec. 3.1.2).

Even though successful, three-dimensional experiments were not suitable for a quantitative investigation of the convective dynamics. This is due to the difficulties in observing the flows inside the porous medium. Each flow visualization experiment requires the time-consuming destructive sampling of the porous medium and the experiment is irrevocably destroyed afterwards.
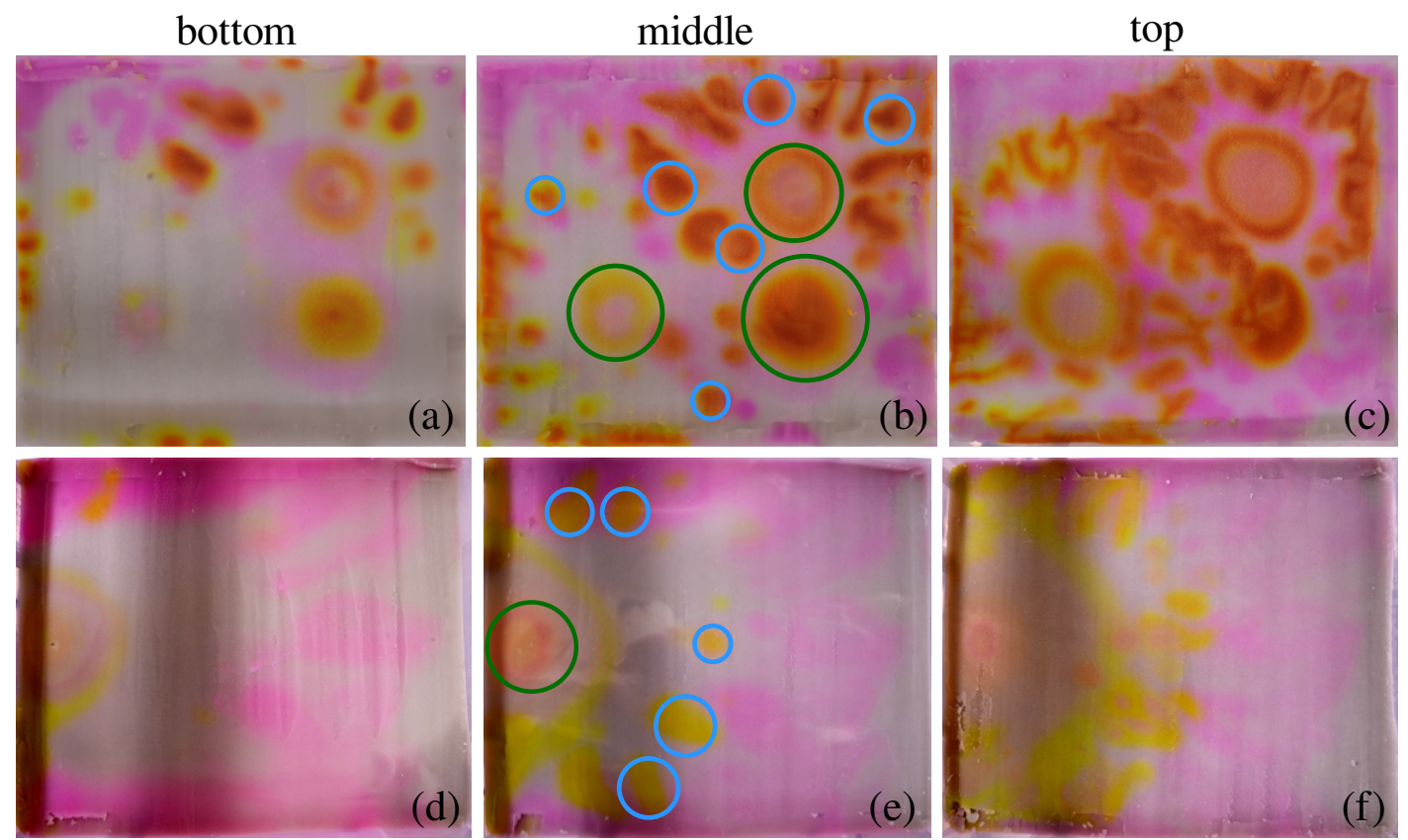

FIGURE 4.10: Two 3D experiments stained with rhodamine and fluorescin and dissected layer by layer to visualize the flows inside the porous medium. Panels (a) and (d) show the layer at $z=18 \mathrm{~cm}$, panels (b) and (e) show the layer at $z=10 \mathrm{~cm}$ and panels (d) and (f) show the layer at $z=2 \mathrm{~cm}$. Upwellings in the middle layer are indicated with green circles, some downwellings have been marked with blue circles for reference. Images courtesy to Birte Thiede.

\subsubsection{Convection in the field}

After finding convective dynamics in model systems of salt playa in the analogue experiments, the next test of my hypothesis is the detection of horizontal concentration gradients indicating the existence of high salinity plumes in the field. To achieve this, I conducted two field campaigns at sites with salt polygons and collected salt concentration profiles from cross sections below polygons, as described in Sec. 3.3. 


\section{Reliability of field results}

As described in Sec. 3.3.1, I used two different methodologies to sample the soil at the field sites: at dryer sites with a water table at approximately $70 \mathrm{~cm}$ I dug trenches whereas at wet sites with a water table right below the salt crust I used a Dutch Gauge Corer. I tested both protocols: the sampling from trenches proved to yield much more reliable results than the sampling with the corer (as discussed below). As a consequence not all results can be reliably used in every context of this work. I was able to reliably calculate Rayleigh numbers for all my field sites, as described in Sec. 4.2.2 and measure pattern wavelengths, as described in Sec. 3.3.2.

Analysis of many of the wet sites showed that the noise introduced by the corer was higher than any signal I expected to see from convective dynamics, but still allowed observation of vertical salinity gradients in many instances. I identified two main sources of noise:

- Salt crystals were pushed into the soil by the corer and subsequently positioned at random depths along the corer. Even small displaced salt crystals are enough to disturb the signal considerably.

- Brine was running down the corer after I pulled it out of the ground. I tried to prevent contamination from water by sampling in a horizontal position and removing an outer layer of approximately $0.5 \mathrm{~cm}$ from the surface of the core prior to sampling. Nevertheless, especially at high permeability soils the core was likely contaminated by brine to a certain degree.

It is next to impossible to quantify the errors pertaining the salt concentration distribution. Nevertheless, I do not expect the measurement of the grain size distribution and therefore permeability of the site to be affected to a large degree, since it is a value averaged between several samples. A discussion of reproducibility of the measurements is given in Sec. 5.4.2. Additionally, during my first field campaign, I sampled the underground with a rather low horizontal resolution. Especially at the Badwater Basin sites, the amount of samples I was allowed to take was restricted and I was not allowed to take samples from a depth deeper than $50 \mathrm{~cm}$. Both low resolution and small depth limited my ability to appropriately resolve the horizontal salt concentration distribution. As a consequence, results from sites where I used the corer and where I collected samples with a horizontal resolution lower than $\Delta X=20 \mathrm{~cm}$ were not used for the analysis of concentration gradients.

\section{Salinity profiles of trench sites}

I collected high resolution salinity maps from three trench sites: T27-S as well as T32-1-L1 (2) and (3). Site T32-2-L1 (1) was sampled with a too low resolution to compile a concentration profile. All three sites were situated in unmanaged areas directly next to managed lake cells, since these areas tended to be drier. Subsurface concentration profiles for the three sites are shown in Figs. 4.11, 4.12 and 4.13. Each 
"pixel" is the result of an individual sample from the field and salinity measurement in the laboratory. White pixels are missing samples that I either did not collect or were which lost during processing.

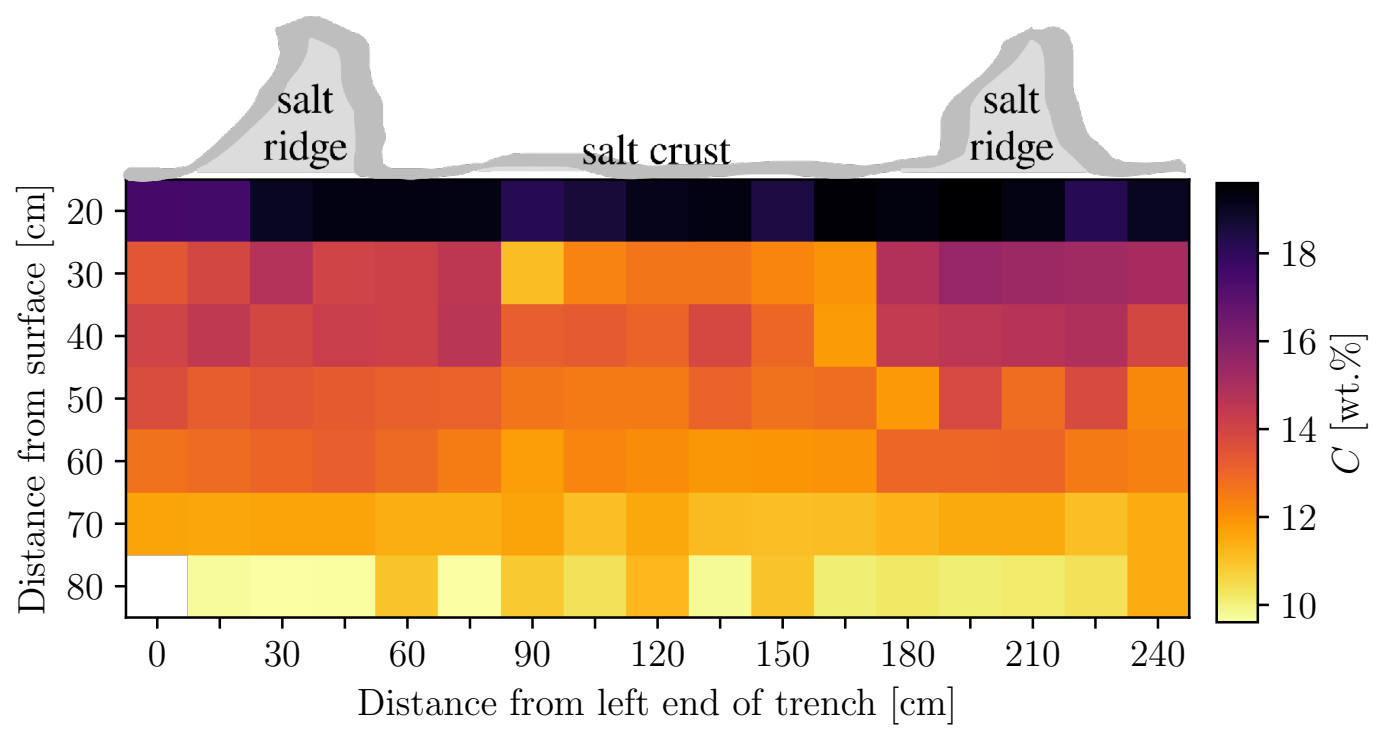

FIGURE 4.11: Salt concentration profile of cross section under a polygon at site T27-S. Ridges were situated at $x=30 \mathrm{~cm}$ and $x=210 \mathrm{~cm}$ respectively. The first two rows of samples are not shown, due to $C>50 \mathrm{wt} . \%$, which is unphysical and indicates contamination with salt crystals from the crust.

For site T27-S I do not display the first two rows of measurements corresponding to $z=2 \mathrm{~cm}$ and $z=10 \mathrm{~cm}$. Both rows showed excessively high salinities of about $2 \rho_{S}$, which is a strong indicator of sample contamination by salt crystals from the crust.

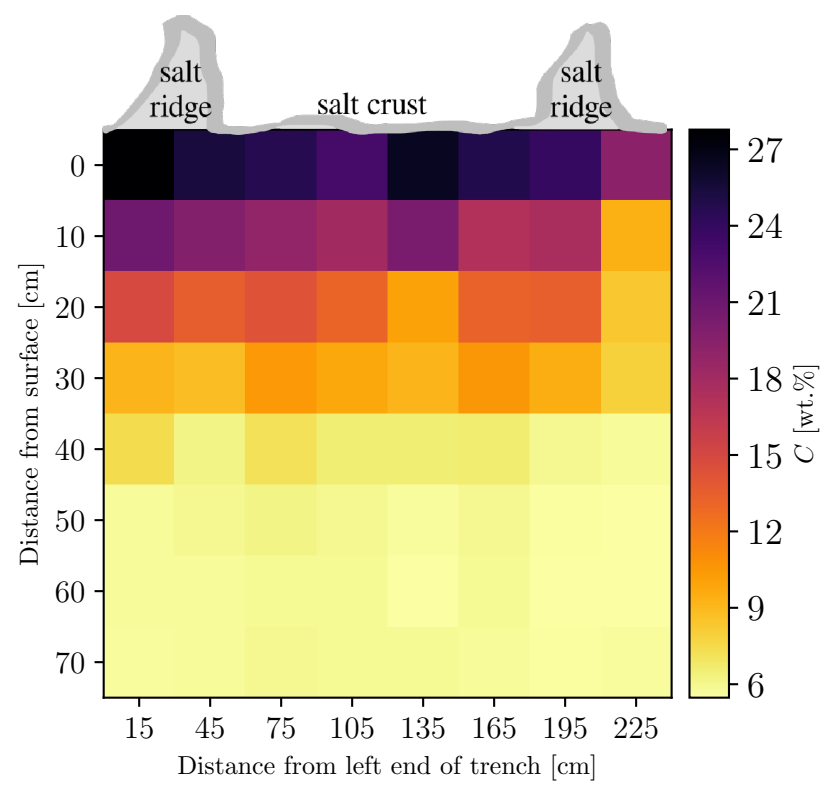

FIGURE 4.12: Salt concentration profile of cross section under a polygon at site T32-1-L1 (2). Ridges were situated at $x=30 \mathrm{~cm}$ and $x=195 \mathrm{~cm}$. 


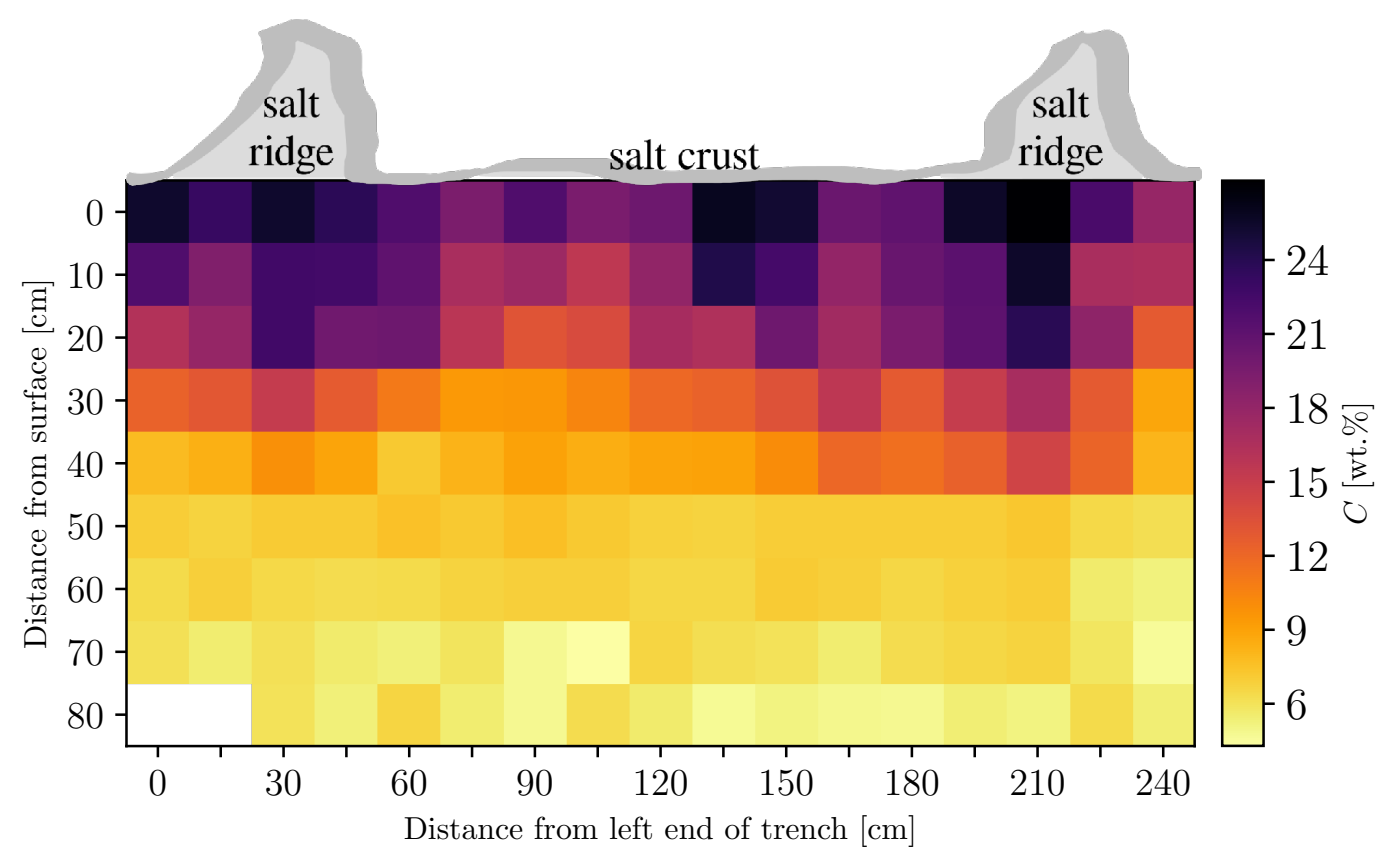

FIGURE 4.13: Salt concentration profile of cross section under a polygon at site T32-1-L1 (3). White pixels indicate missing measurements. Ridges were situated at $x=30 \mathrm{~cm}$ and $x=225 \mathrm{~cm}$.

I processed the samples from site T32-1-L1 (2) in two batches: one batch contained the samples extracted at positions $x \in\{0,30,60,90,120,150,180,210\} \mathrm{cm}$, the other the samples from positions $x \in\{15,45,75,105,135,165,195,225\} \mathrm{cm}$. During the determination of salt concentrations of samples from the first batch in the laboratory, I made a mistake in step (3) of the processing protocol (see Sec. 3.2.4): I did not execute the second dilution step correctly but did not notice the mistake until after the processing was completed and the samples were destroyed. I therefore only use the samples from the second batch, which is why the salinity profile shown in Fig. 4.12 only shows eight columns of samples, even though 16 were collected.

Sites T27-S and T32-1-L1 (3) clearly show plumes of higher salt concentration below the ridges down to a depth of about $40-60 \mathrm{~cm}$. For site T32-1-L1 (2), the plumes are not that clearly discernible. Plumes show a salt concentration which is increased by a factor of 2-4 over the background salt concentration. For all three sites, a clear salt concentration gradient from high salinity near the surface to uniform and low salinity at depth is visible.

\section{Statistical significance}

To quantify above observations, I calculate the statistical significance of the difference between the salt concentration measurements below the ridges as compared to the measurements below the center of the polygon. Specifically, I divide the measurements into two sets of measurements as visualized in Fig. 4.14: 
set 1 All samples from the column directly below the ridges as well as two columns left and right of the ridges for a total of 69,40 and 88 samples for sites T27-S, T32-1-L1 (2) and T32-1-L1 (3) respectively (light blue regions in the figure).

set 2 All other samples from the middle of the polygons, for a total of 49, 24 and 63 samples for sites T27-S, T32-1-L1 (2) and T32-1-L1 (3) respectively (white region).

My testing hypothesis is:

The samples belonging to set 1 and set 2 are drawn from a single salinity distribution:

$$
H_{0}: F_{1}(\text { ridge })=F_{2}(\text { center }) \text {. }
$$

The alternative hypothesis is:

The samples belonging to set 1 and set 2 are drawn from different distributions:

$$
H_{1}: F_{1} \text { (ridge) } \neq F_{2} \text { (center) } \text {. }
$$

I normalize the salt concentration measured at each sample by the average salt concentration measured at a given depth $z$, to account for the steep decrease of salt concentration with depth and make the different depth comparable. The normalized salt concentration distributions along the $x$-direction are shown in Fig. 4.14 (a) to (c). Different sampling depths $z$ are color coded from yellow (close to the crust) to purple (far away from the crust).

I perform a two-sided Kolmogorov-Smirnov test [171] on the normalized salt concentrations of the two sets of samples for each site to test the hypothesis $H_{0}$. For all three sites I find that the hypothesis is not valid with $p$-values of $p=0.005$, $p=0.050$ and $p=0.019$ for sites T27-S, T32-1-L1 (2) and T32-1-L1 (3) respectively. I therefore reject $H_{0}$ and conclude, that the samples from below ridges and from below centers are drawn from two different distributions. I interpret this as a clear indication that the conditions in the center of the polygon, which affect the salt concentration distribution, are different from the conditions below the ridges. Based on the concentration profiles I conclude that there are upwellings in the polygon centers whereas below the ridges, the flow of water points downwards, in the direction of gravity.

\subsubsection{Summary}

I successfully set up experiments across a range of Ra similar to the conditions found in the field, but with an evaporation rate that is about three orders of magnitude higher than the evaporation rate in nature. This allows me to model and observe the system on time-scales that are experimentally more easily accessible and on finer length scales. 

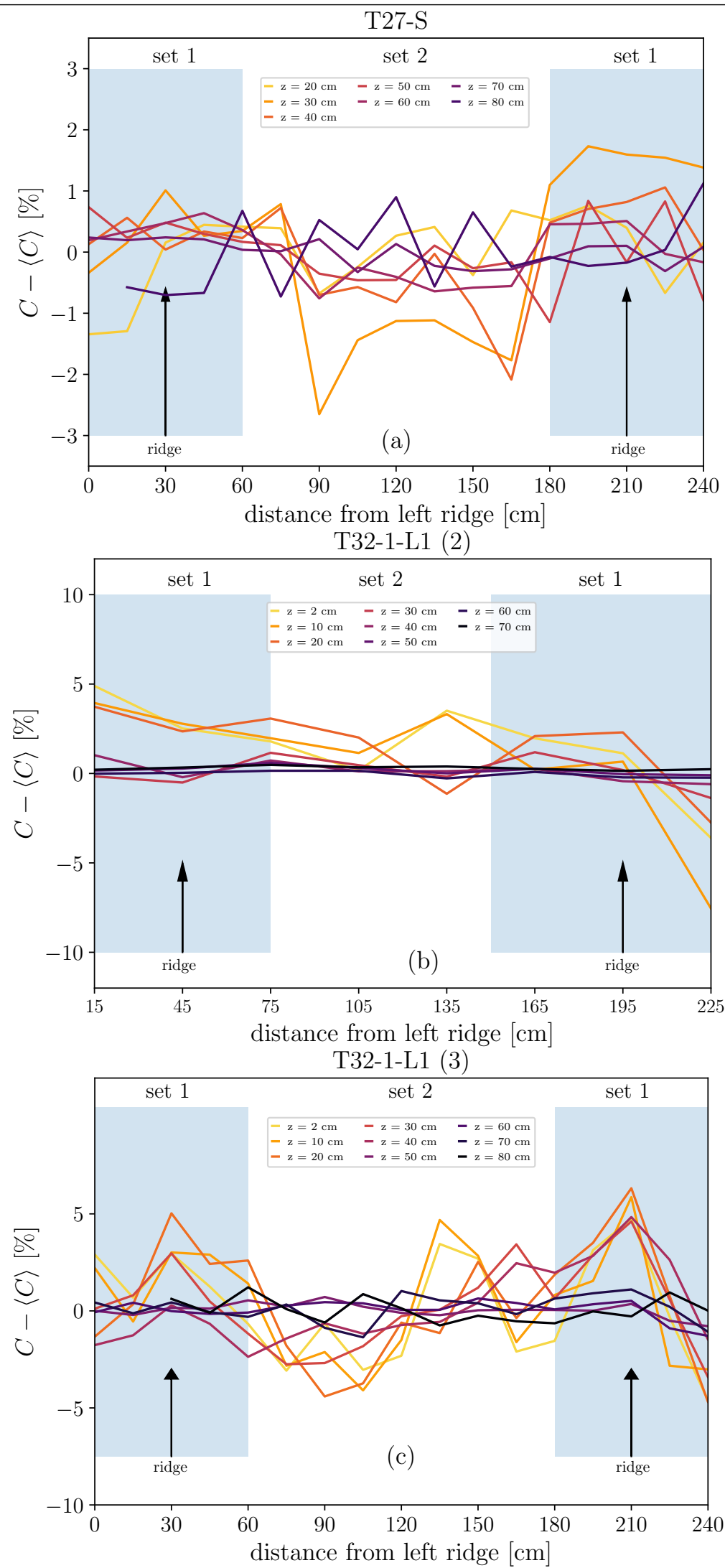

FIGURE 4.14: Salt concentration $C$ normalized by average salt concentration $\langle C\rangle$ for sites T27-S, T32-1-L1 (2) and T32-1-L1 (3). Positions of ridges are indicated with arrows and data points belonging to set 1 are indicated by a blue background. 
By conducting experiments in Hele-Shaw cells filled with a porous medium I was able to confirm that convective dynamics set in once the system Rayleigh number surpasses a critical value. Furthermore I confirmed that the observed fluid flow inside the cells is indeed coupled to concentration gradients. Finally I presented some proof-of-principle experiments in three dimensional setups to confirm that the simplification of the system to a two-dimensional problem does not change the qualitative behavior of the dynamics.

I was able to collect samples with a high enough resolution and accuracy allowing for the detection of horizontal concentration gradients at a total of three field sites. I find statistically significant evidence for plumes of high salinity at all three sites. Plumes are co-located with the ridges which were visible at the surface. This is very strong evidence that the polygonal salt ridge patterns are linked to subsurface dynamics, which is driven by density gradients due to high salinity.

\subsection{Scaling}

In Sec. 4.2 and Sec. 4.4 I was able to demonstrate that salinity driven convection occurs in settings as found in salt deserts. I also showed evidence of plumes of high salinity which are co-located with the patterns visible at the surface. What is still missing is an explanation of the pattern length scale that is found in nature.

Most notably, the length scale of the patterns does not change much from Chile to Iran to the West coast of the US, even though the environmental circumstances change drastically. In the following section I am going to present my experimental and numerical investigation of the length scale of the convective dynamics, i.e. the spacing between the high salinity plumes. Furthermore, I will present my investigation of the significant coarsening behavior I found in the simulations and how coarsening could explain the pattern length scales observed in the experiment and in nature.

Simulations conducted for this investigation were implemented as two-dimensional systems scaled by the Rayleigh number, as described in Sec. 3.1.

\subsubsection{Scaling of wavenumber with Rayleigh number}

In Sec. 4.2.1 I presented the results of the linear stability analysis of the system, which show a clear dependence of the dimensionless wavenumber $a$ of the most unstable mode on Ra. As discussed in Sec. 4.2.1 and shown in Fig. 4.15 (dotted gray line), the wavenumber of the most unstable mode has a clear dependence on the Rayleigh number. $a$ increases by a factor of 20 as the Rayleigh number increases from about $\mathrm{Ra}_{c}=14.35$ to $\mathrm{Ra}=4 \cdot 10^{3}$. I investigated this prediction in my numerical simulations of the system: I simulated the system in a wide range of Rayleigh numbers from $\mathrm{Ra}=2 \cdot 10^{1}$ to $\mathrm{Ra}=4 \cdot 10^{3}$ and measured the wavenumber of the ensuing convective dynamics as described in Sec. 3.1.8. 
In Fig. 4.15 I show the behavior of the wavenumber with Ra at depth $Z=-1$ and time $\tau=\tau_{S}$. Measurements are averages and standard deviations of ensembles of 5-10 simulation runs. Theoretical predictions for the neutral stability curve and most unstable mode only reach values of $a \approx 10$, since for higher Rayleigh numbers the algorithm to solve the eigenvalue problem (see Sec. 4.2.1) does not converge anymore. I

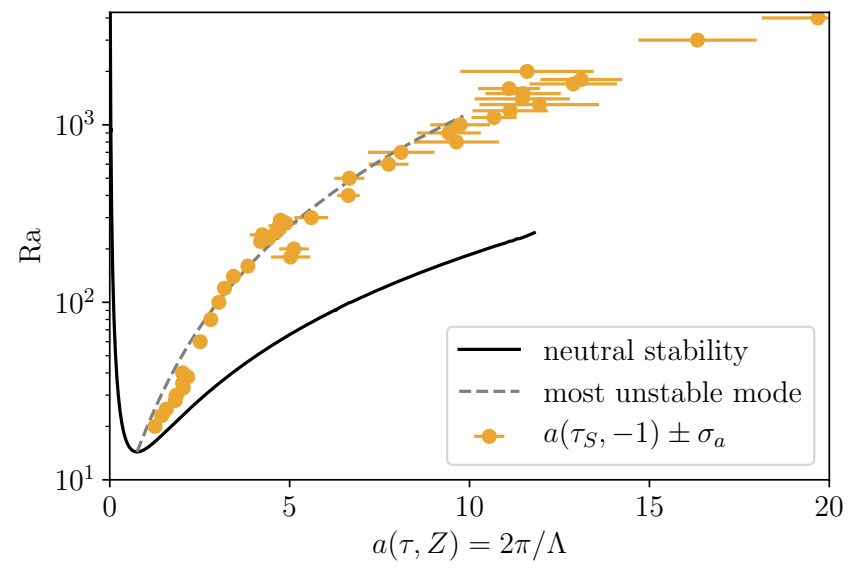

FIGURE 4.15: Wavenumber $a$ and standard deviation $\sigma_{a}$ measured for simulations with $\mathrm{Ra}=20-4000$ at short times $\tau=\tau_{S}$. Measurements are ensemble averages over 5-10 runs for every Ra.

find that the simulation closely follows the theoretical prediction of increasing wavenumber with increasing Ra.

\section{Qualitative coarsening}

Visual inspection of the system as time increases reveals that, after a short period of plume growth in vertical direction, plumes start moving in a horizontal direction and coalesce into a few larger plumes which sink downwards. As a result, the wavelength of the high salinity plumes rapidly diverges from the wavelength of the most unstable mode.

In Fig. 4.16 I visualize this behavior for an exemplary simulation at $\mathrm{Ra}=100$, measured at different depths between $Z=-1$ and $Z=-90$. In the beginning, the wavenumber close to the top boundary (yellow line) is close to the value of $a=3$ predicted by the linear stability analysis for the most unstable mode (see Fig. 4.1). The wavenumber then quickly drops to a value of approximately $a=1.5$ at time $\tau=5 \mathrm{~T}$. Subsequently, wavenumbers measured close to the top boundary (yellow and orange lines) oscillate between approximately $a=1$ and $a=2$, as small plumes re-emerge at the top boundary and are swept into larger plumes again. At a time of $\tau \approx 10 \mathrm{~T}$, the first plume reaches the bottom boundary. Wavenumbers measured deeper down in the system (red and purple lines) are measurable only after the first plumes reached the respective depths, and saturate at the wavenumber of the coarsened system around $a=0.5$ for larger times $\tau \geq 15 \mathrm{~T}$.

This behavior has already been considered in the context of $\mathrm{CO}_{2}$ sequestration and was phenomenologically described in great detail by [73] for different boundary conditions (see Sec. 5.2.1 for a detailed discussion). The coarsening becomes even more apparent when the simulations are visualized in spacetime diagrams showing the development of the salinity $S$ at a given depth $Z=-1$ with time. The spacetime diagram shown in Fig. 4.17 (a) for a run at $\mathrm{Ra}=100$ shows clearly that smaller plumes originate at the surface and are swept into two larger plumes repeatedly. The 


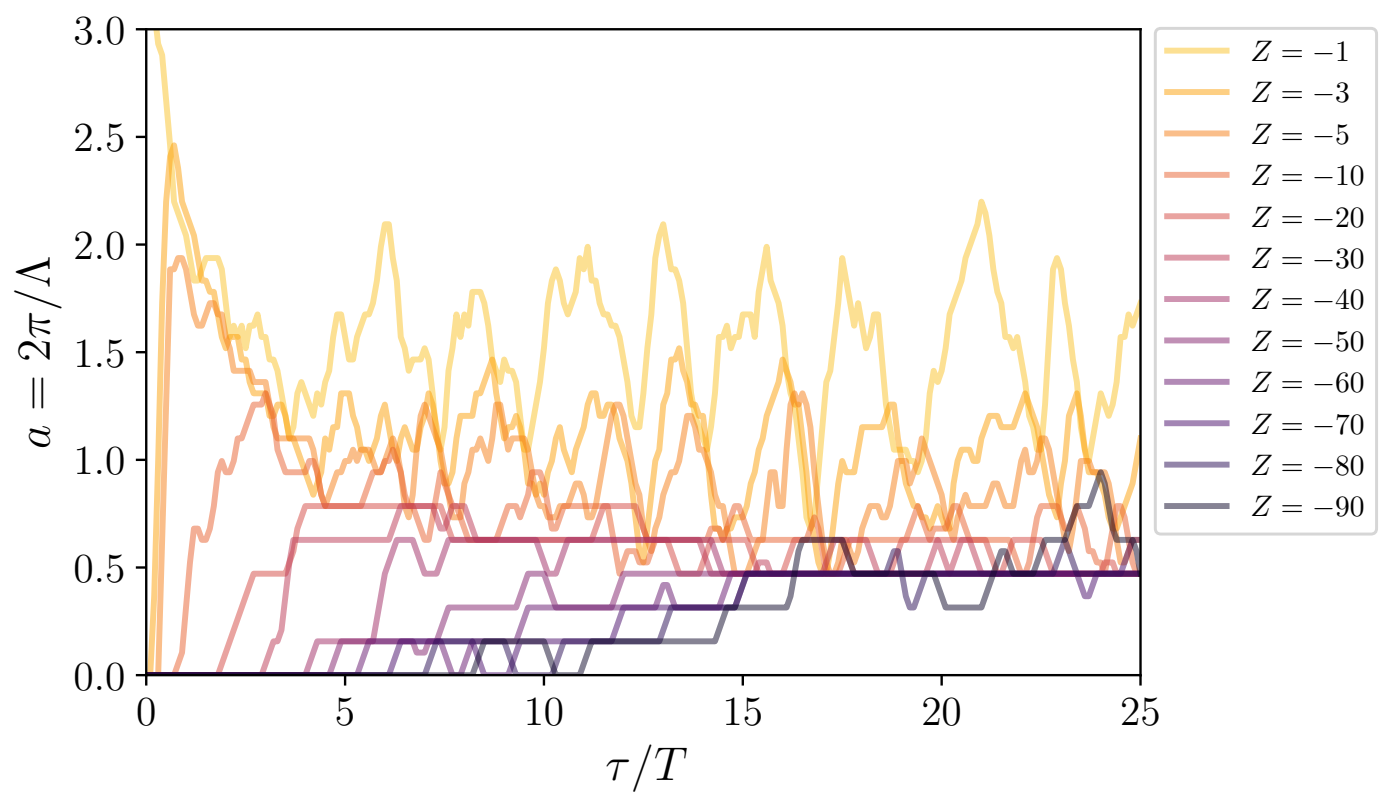

FIGURE 4.16: Wavenumber development over time in a simulation at $\mathrm{Ra}=100$. Wavenumbers were measured at different depths, depths are color-coded from yellow $(Z=-1)$ to purple $(Z=-90)$.

positions - subsequently called roots - of the two large plumes fluctuate in space as time passes. For Fig. 4.17 (b) this behavior is less pronounced due to the smaller Rayleigh number $(\mathrm{Ra}=38)$ but the coarsening is still clearly visible. This observation leads to two questions which I will try to answer in the following:

- How does the length scale of the coarsened dynamics scale with Rayleigh number and time?

- If the roots of the large downwelling plumes fluctuate, how can they drive the emergence of (spatially stationary) patterns at the surface?

\section{Quantifying coarsening in simulations}

In Fig. $4.18 \mathrm{I}$ show how the wavenumber measured at system ages of $\tau_{S}, 10 \tau_{S}$ and $50 \tau_{S}$ behaves for simulations with $\mathrm{Ra}=2 \cdot 10^{1}-2 \cdot 10^{3}$. For the two higher system ages I measured wavenumbers at $Z=-10$ instead of $Z=-1$, to capture the coarsened dynamics rather than the constantly emerging small plumes at the surface.

It is clearly visible that the dependence of the wavenumber on Ra reduces for larger times $\tau=10 \tau_{S}$ (red dots). Wavenumbers for a wide range of Rayleigh numbers cluster in the range of $a=0.2-2.5$. For even longer times $\tau=50 \tau_{S}$ (gray dots), the coarsening continues but slows down significantly. For stability reasons and computational cost (see Sec. 3.1.7), I was only able to run all simulations for higher relative ages for single Rayleigh numbers.

In Fig. 4.19 I show the wavenumber development at $\mathrm{Ra}=200$ and $Z=-5$ for a range of relative system ages between one $\tau_{S}$ and $99 \tau_{S}$. The values shown are 

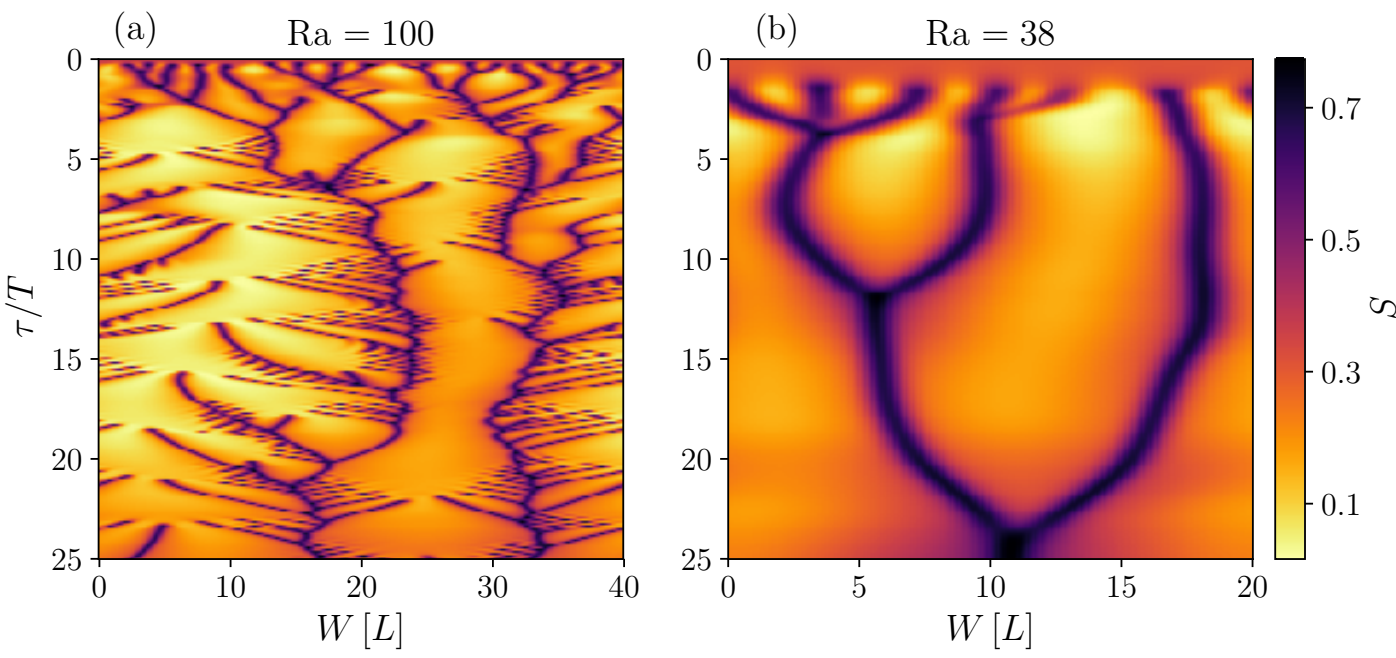

FIGURE 4.17: Development of the salinity distribution at $Z=-1$ for (a) $\mathrm{Ra}=100$ and $(\mathrm{b}) \mathrm{Ra}=38$. Coarsening is clearly visible for longer times.

averages over an ensemble of 9 runs (red line) and the standard deviation (shaded red area). The blue line is a linear fit to the wavenumber values measured at ages $\tau=50 \tau_{S}$ to $\tau=99 \tau_{S}$. The linear fit yields $a\left(\tau_{S}\right)=1.57+(0.002 \pm 0.01) \tau_{S}$. This indicates that the coarsening of the dynamics as ceased at a relative system age of $\tau \approx \tau_{S}$. Nevertheless, at such long simulation times, plumes have reached the bottom of the simulated domain and information about the bottom boundary starts to propagate through the system, which could yield to results which diverge from the natural setting, where I assume an infinitely deep system. Therefore, these results have to be taken with caution.

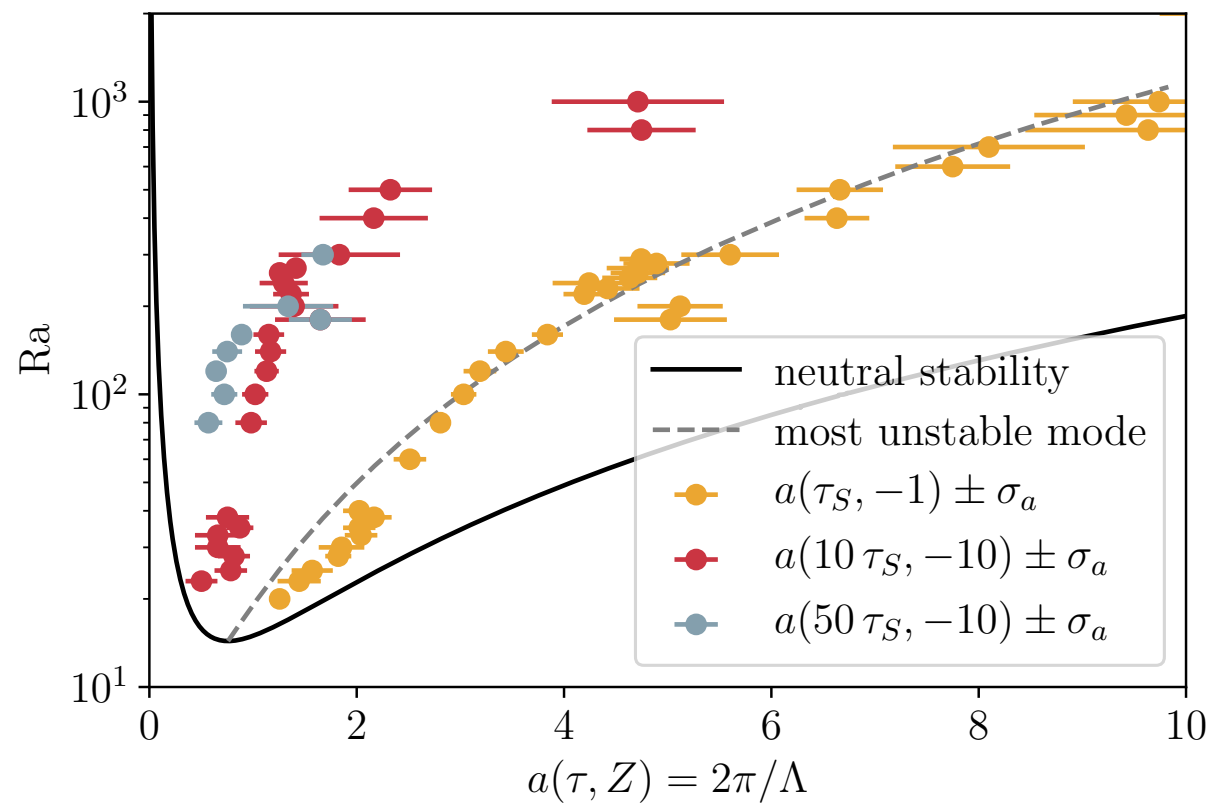

FIGURE 4.18: Wavenumbers and standard deviations for simulations for a large range of $\mathrm{Ra}=2 \cdot 10^{1}-2 \cdot 10^{3}$ and $\tau=\tau_{S}$ (yellow), $\tau=10 \tau_{S}$ (red) as well as $\tau=50 \tau_{S}$ (gray). Measurements are ensemble averages over 5-10 runs for every Ra. 


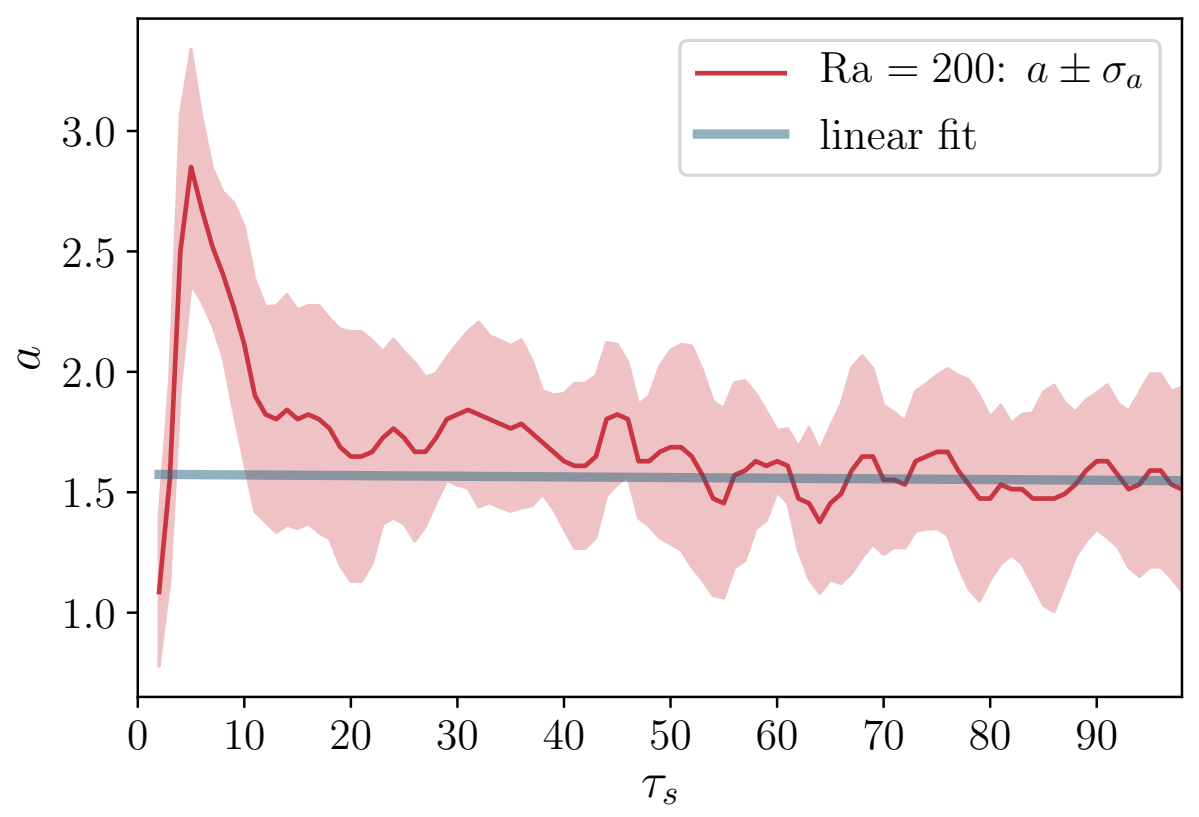

FIGURE 4.19: Wavenumber $a$ measured at $Z=-5$ for simulations at $\mathrm{Ra}=200$ and relative system ages $\tau=\tau_{S}$ to $\tau=99 \tau_{S}$. Measured values (red line) and uncertainty (shaded red area) are averages and standard deviations of an ensemble of 9 runs. The blue line is a linear fit to the wavenumbers measured between $\tau=50 \tau_{S}$ and $\tau=99 \tau_{S}$.

\subsubsection{Wavenumbers in experiments}

In the experiment, I was able to drive the dynamics much faster than in the field and explore the behavior up to much larger natural times than possible in the simulations, since the simulations would have taken too long to complete. By varying the evaporation rate in the range of $E=0.34 \cdot 10^{-7} \mathrm{~m} / \mathrm{s}$ to $E=11.7 \cdot 10^{-7} \mathrm{~m} / \mathrm{s}, \mathrm{I}$ achieved characteristic length scales of $L=0.14 \cdot 10^{-2} \mathrm{~m}$ to $L=4.69 \cdot 10^{-2} \mathrm{~m}$ and natural time scales of $T=9 \mathrm{~min}$ to $T=7$ days. I let experiments coarsen for several weeks and measured the system wavenumber throughout. I calculate $\tau_{S}$ for each system using Eq. (4.9) to estimate the relative system age at the time of measurement. For some systems, the wavelength was measured at system ages as high as $\tau=5.3 \cdot 10^{4} \tau_{S}$

In Fig. 4.20 (a) I show the wavenumbers measured in the experiment over the Rayleigh number, color coded by the logarithm of the system age. It is noticeable that early / high Rayleigh number measurements (dark green) fall in the same range of $a=0.5-1.5$ observed in the simulations. Later measurements (light green) approach the neutral stability curve predicted by theory. Figure 4.20. I have to note that there is a correlation between measurements with low Ra (and high $E$ ) and high system age due to the experimental protocol: I started with low evaporation rates in the beginning and for the first coloring experiments in each setup, and then increased the evaporation rate for the later coloring experiments. Figure 4.20 (b) shows wavenumbers over the logarithm of the system age. It is not clear, if the coarsening of the wavenumbers is caused by higher system age or the lower Rayleigh number. 

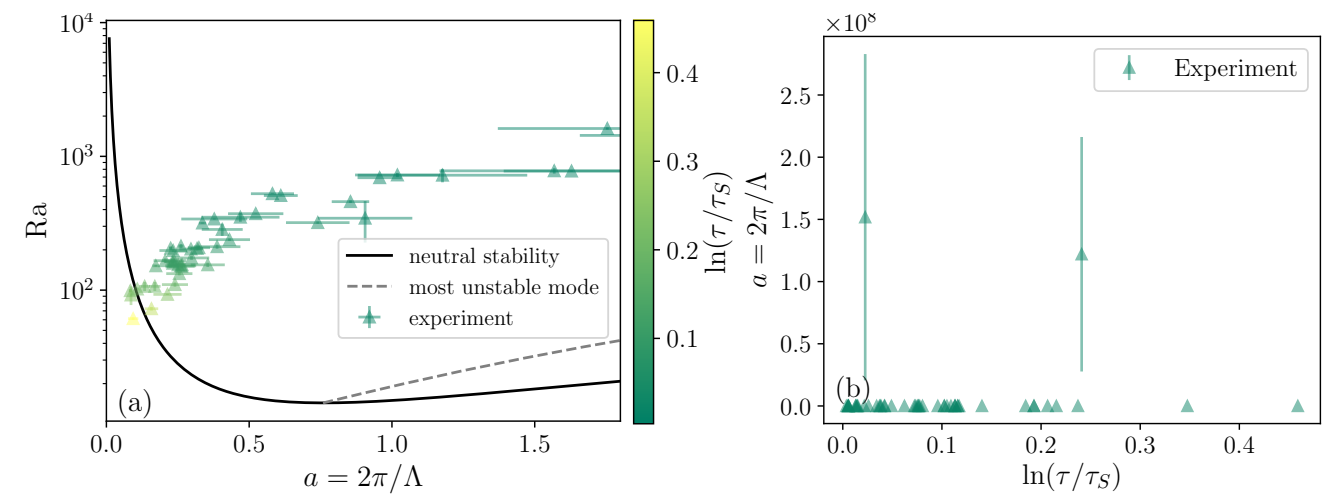

FIGURE 4.20: (a) Wavenumbers measured in the experiment over Rayleigh numbers color coded by the logarithm of the relative system age $\tau$ expressed in $\tau_{S}$. (b) Wavenumbers over the logarithm of the relative system age $\tau / \tau_{S}$.

\subsubsection{Pattern length scales in the field}

Next to the measurements of the Rayleigh numbers at each field site, the dimensional pattern wavelength $\lambda$ and dimensionless wavenumber $a=2 \pi L / \lambda$ are necessary to investigate the scaling of $a$ in the field and compare it to my findings in the simulations and experiments described above. During my two field campaigns I performed high accuracy scans of the surface relief of the salt polygons using a TLS. I determine the dominant wavelength of the pattern from the scans (see Sec. 3.3.2 for details).

From these scans, I calculated wavelengths and dimensionless wavenumbers as described in Sec. 3.3.2 and following [154]. Wavenumbers over the Rayleigh numbers measured at the field sites (listed in Tab. 4.3) are shown in Fig. 4.21 (a) (Owens Lake: blue, Badwater Basin: red, Bristol Playa: yellow). Additionally, measured wavelength $\lambda$ and calculated dimensionless wavenumber $a$ are listed in Tab. 4.7. Scans of two exemplary sections of $10 \times 10 \mathrm{~m}$ each, from sites T27-S and T32-1-L1 (3), from which wavelengths were extracted, are shown in Fig. 4.21 (b) and (c). No separate scan was made for site Badwater Basin (2), which was located very close to Badwater Basin (1). For sites T32-1-L1 (2) and (3), only one scan was performed and $10 \times 10 \mathrm{~m}$ regions in the north and south of the scan, close to the sampled polygons were extracted, from which wavelength and ridge height was extracted.

Wavenumbers for Owens Lake cluster in the range $a=0.5-2$ and do not noticeably scale with measured Rayleigh number - as expected from my original claim that the pattern wavelength $\lambda$ is robust around the world. together with my analysis of the properties of the salinity gradient (see Sec. 4.3.2), this could mean, that the Rayleigh number in the boundary layer directly below the salt crust is effectively reduced by the vigorous convection in the system and therefore all wavenumbers fall in approximately the same range.

Wavenumbers for Badwater Basin are higher, between $a \approx 3$ and $a \approx 7$. These sites also have Rayleigh numbers around $\mathrm{Ra} \approx 3 \cdot 10^{3}$, which is on the high end of the measured spectrum of Rayleigh numbers for all field sites. Taken together, 


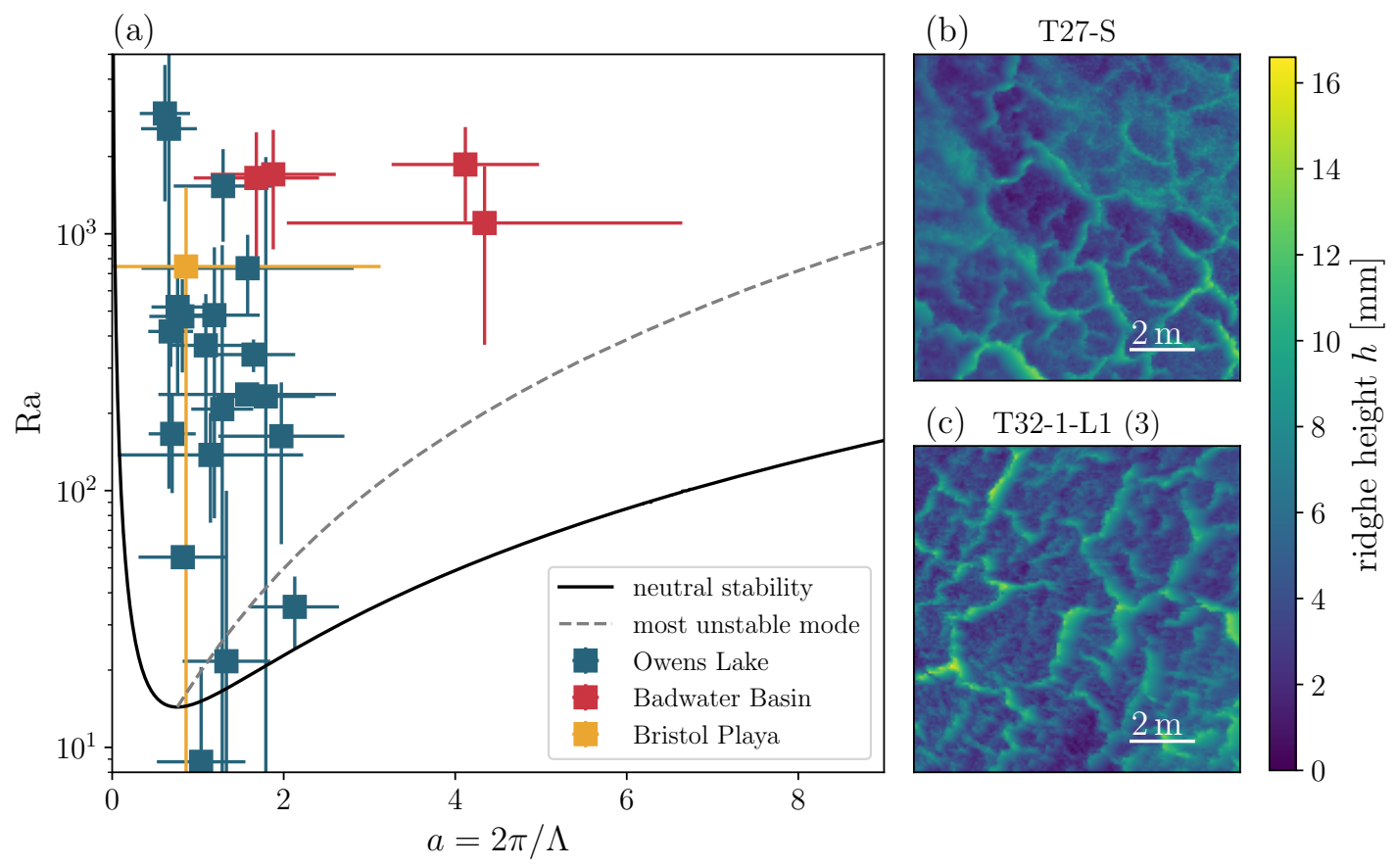

FIGURE 4.21: (a) Wavenumbers over Rayleigh numbers measured in the field at Owens Lake (blue), Badwater Basin (red) and Bristol Playa (yellow). (b), (c) Surface relief measured at two of the trench sites.

the wavenumbers from Owens Lake and Badwater Basin indicate a trend towards increasing wavenumbers for very large Rayleigh numbers. Nevertheless, since uncertainties are large and the number of data points is small, a reliable conclusion about the trend of $a(\mathrm{Ra})$ does not seem warranted. The wavenumber measured at the Bristol Playa site falls in the same range of wavenumbers measured at Owens Lake.

For the sites at Badwater Basin, a trend of decreasing wavelengths with distance from the shore of the salt flats is noticeable as wavelengths drop from $\lambda=1.42 \mathrm{~m}$ at site (1) about $100 \mathrm{~m}$ inwards from the shore to $\lambda=0.55 \mathrm{~m}$ at site (5), about $400 \mathrm{~m}$ inwards from the shore.

As described in Sec. 3.3.2, information about the average height of the ridges at each field site can also be extracted from the surface relief scans. Ridge heights can be interpreted as an indicator of the age of a ridge, since ridges grow with time as salt precipitates. A general scaling is hard to predict, because it will depend on the mechanics of ridge growth and erosion of ridges by wind. Nevertheless, a general correlation of ridge height with ridge age seems intuitive.

In Tab. 4.7 I list the $90^{\text {th }}$ percentile ridge height $h$ next to the wavelength for each site. Ridge heights range between $h=2 \mathrm{~cm}$ and $h=15 \mathrm{~cm}$ for Owens Lake and $3 \mathrm{~cm}$ to $8 \mathrm{~cm}$ for Badwater Basin. Ridges at Badwater Basin have an average height of $\langle h\rangle=5.3$, which is slightly smaller than the ridges at Owens Lake with $\langle h\rangle=6.7$. In Fig. 4.22 I show the ridge height over the wavelength or all field sites. Ridge heights correlate positively with polygon wavelength (Pearson correlation coefficient $\rho(h, \lambda)=0.55, p=0.004)$. This is consistent with the notion that the 


\begin{tabular}{l|c|c|c} 
Site & $\lambda[\mathrm{m}]$ & $a=2 \pi L / \lambda$ & $h\left[10^{-3} \mathrm{~m}\right]$ \\
\hline Badwater Basin (1) & $1.42 \pm 0.58$ & $1.68 \pm 0.74$ & $7.7 \pm 2.8$ \\
Badwater Basin (3) & $1.27 \pm 0.55$ & $1.88 \pm 0.86$ & $7.1 \pm 2.6$ \\
Badwater Basin (4) & $0.58 \pm 0.32$ & $4.12 \pm 2.31$ & $2.8 \pm 1.3$ \\
Badwater Basin (5) & $0.55 \pm 0.28$ & $4.44 \pm 2.27$ & $3.4 \pm 1.4$ \\
\hline Bristol Playa & $2.15 \pm 0.86$ & $0.86 \pm 0.37$ & $11.4 \pm 4.2$ \\
\hline T10-3 & $1.79 \pm 0.86$ & $1.04 \pm 0.52$ & $7.4 \pm 3.1$ \\
T16 & $1.39 \pm 0.51$ & $1.33 \pm 0.52$ & $7.5 \pm 2.8$ \\
T2-4 & $1.13 \pm 0.54$ & $1.65 \pm 0.81$ & $2.5 \pm 1.0$ \\
T2-5 (1) & $1.04 \pm 0.41$ & $1.79 \pm 0.74$ & $4.5 \pm 1.6$ \\
T2-5 (2) & $0.94 \pm 0.50$ & $1.97 \pm 1.09$ & $2.6 \pm 1.5$ \\
T2-5 (3) & $1.62 \pm 0.65$ & $1.15 \pm 0.49$ & $4.4 \pm 1.6$ \\
T25-3 (1) & $1.18 \pm 0.89$ & $0.82 \pm 0.35$ & $6.2 \pm 2.2$ \\
T25-3 (2) & $2.25 \pm 0.56$ & $1.57 \pm 0.78$ & $15.3 \pm 5.1$ \\
T27-A (1) & $1.70 \pm 0.65$ & $1.09 \pm 0.45$ & $5.0 \pm 1.8$ \\
T27-A (2) & $2.72 \pm 0.98$ & $0.68 \pm 0.27$ & $7.6 \pm 2.5$ \\
T27-A (3) & $1.45 \pm 0.55$ & $1.28 \pm 0.52$ & $7.3 \pm 2.4$ \\
T27S & $1.51 \pm 0.64$ & $1.23 \pm 0.55$ & $6.5 \pm 2.4$ \\
T29-3 (1) & $3.02 \pm 1.40$ & $0.61 \pm 0.30$ & $7.3 \pm 3.1$ \\
T29-3 (2) & $2.80 \pm 1.34$ & $0.66 \pm 0.33$ & $6.7 \pm 3.0$ \\
T32-1-L1 (1) & $1.56 \pm 0.66$ & $1.19 \pm 0.53$ & $13.8 \pm 4.8$ \\
T32-1-L1 (2) & $2.65 \pm 0.98$ & $0.70 \pm 0.28$ & $10.8 \pm 3.6$ \\
T32-1-L1 (3) & $2.43 \pm 0.92$ & $0.76 \pm 0.31$ & $7.8 \pm 2.8$ \\
T36-3 (1) & $1.17 \pm 0.91$ & $1.58 \pm 1.24$ & $2.2 \pm 2.2$ \\
T36-3 (2) & $2.27 \pm 1.03$ & $0.82 \pm 0.39$ & $7.2 \pm 3.4$ \\
T36-3 (3) & $1.93 \pm 0.62$ & $1.29 \pm 0.58$ & $4.9 \pm 1.9$ \\
T8-W & $0.87 \pm 0.41$ & $2.13 \pm 1.54$ & $3.9 \pm 1.5$ \\
\hline
\end{tabular}

TABLE 4.7: Pattern wavelength $\lambda$, dimensionless wavenumber $a$ and ridge height $h$ measured by TLS for most of the field sites at Owens Lake (filled squares) and Badwater Basin and Bristol Playa (open squares).

selected wavelength coarsens with time. Therefore, as polygons grow older, their ridge height and wavelength both increase.

\subsubsection{Summary}

Based on numerical simulations of the buoyancy driven convective dynamics, I have shown that in older systems the scaling of wavenumbers with Rayleigh numbers is weaker than the scaling predicted in Sec. 4.2.1 for the most unstable mode. The downwelling plumes tend to coarsen and, while small plumes with a higher wavenumber still originate at the top boundary, they quickly coalesce into a few larger plumes as they sink downwards. In both the experiment and the field I find wavenumbers in the same range that is associated with a significantly coarsened system at a relative age of $10 \tau_{S}$.

An overview of the wavenumbers measured in simulations, experiment and the field is shown in Fig. 4.23. I quantified the coarsening behavior with simulations up to $\tau=50 \tau_{S}$ and found that for a large range of $\mathrm{Ra}$, wavenumbers tend to cluster in 


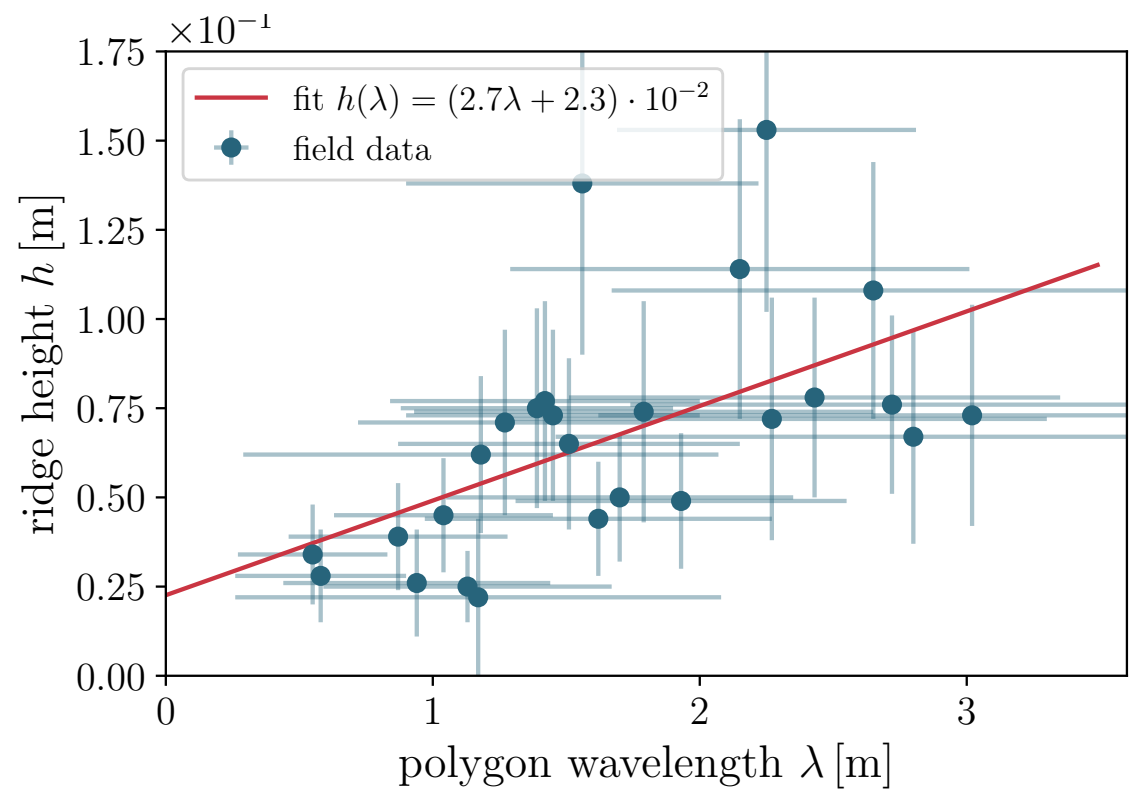

FIGURE 4.22: Ridge height and polygon wavelength measured by TLS in the field.

the range $a=0.2-2.5$. In the experiments, I measured wavenumbers in even older systems up to $\tau=5.3 \cdot 10^{4} \tau_{S}$ and found even smaller dimensionless wavenumbers in the range of $a=0.1-1.5$.

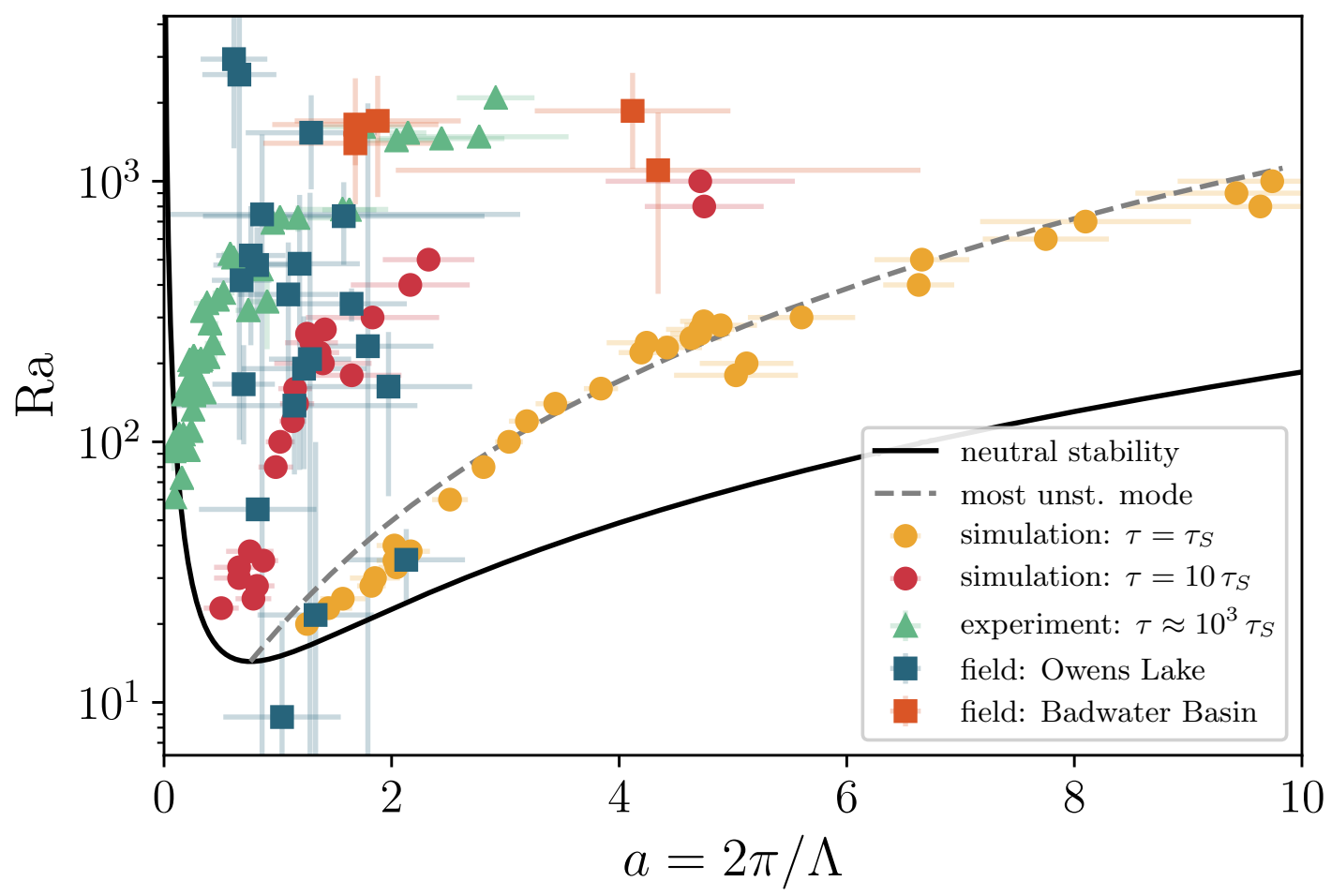

FIGURE 4.23: Overview plot of dimensionless wavenumbers measured in the field (blue squares, Owens Lake and Bristol Playa, orange squares Badwater Basin), the experiments (green triangles) and the simulations at early (yellow dots) and late (red dots) times. The lines indicate the theoretically predicted neutral stability curve (black solid line) and the most unstable mode (gray dotted line). 
Wavenumbers measured in the field at Owens Lake fall into the range of $a=0.5-2$. This places them in-between the wavenumbers measured at $\tau=10 \tau_{S}$ in the simulations and those measured in the experiments for very old systems. Wavenumbers at Badwater Basin show higher values between $a=2$ and $a=4.5$. This can be interpreted as a continuation of the $a(\mathrm{Ra})$ trend predicted by the theory for the most unstable mode, and the simulations at intermediate ages of $10 \tau_{S}$. On the other hand, the discrepancy between the wavenumbers measured at Owens Lake and Badwater Basin could also be due to some still unknown parameter that influences the selected wavelength in the field, and is different between the two field sites.

For Owens Lake, one natural time $T=D / E^{2}$ corresponds to about 675 days, if the adjusted diffusion coefficient as discussed in Sec. 4.3.4 is used. The median characteristic time for the field sites calculated based on their Rayleigh number and the scaling given in Eq. (4.9) is $\tau_{S}=0.05 \mathrm{~T}$. This corresponds to approximately 34 days in the field. Therefore, after a flooding event, the convective dynamics set in on a time scale that is comparable to other geomorphological processes such as dune development [172]. Nevertheless, the time scale is very low when compared to other processes that produce polygonal patterns, such as patterned ground [173], which takes thousands of years to form.

Both Owens Lake and Badwater Basin had recorded flooding events about one year prior to my visit. If the polygons I visited were approximately one year old, this would place their age at about $11 \tau_{S}$ and well within the range of wavenumbers I would expect in a system which had coarsened considerably. While my estimate for the age of the polygons based on their wavelength is very rough, it is consistent with the age estimate based on the flooding events.

Additional evidence for the coarsening of polygon wavelengths can be found in the work of [8]. Wavelengths of ridges were measured in two consecutive years at the Makgadikgadi Pans, Botswana. Wavelengths at almost all sites increased significantly over the course of a year. Wavelengths on average increased by a factor of approximately three over the course of a year. This indicates that, while initially ridges with a smaller wavelength may be formed, a larger wavelength is selected as the crust ages and only certain ridges continue to grow. Other ridges shrink again, due to weathering by wind.

As I showed in Sec. 4.4.2, high salinity plumes are co-located with ridges at the surface. Nevertheless in the simulations I found that the roots (i.e. the location where they touch the top boundary) of the large downwelling plumes slowly fluctuate in space as the system ages. This is seemingly contradictory with the need for the plumes to stay stationary below the ridges for long enough to support ridge growth. To resolve this apparent contradiction, in Sec. 4.6 I will introduce a feedback mechanism which is able to pin the plumes in space. 


\subsection{Feedback}

During inspection of the space-time diagrams of the simulations I observed spatial fluctuations of the high salinity plumes with time (see Fig. 4.17). This seemingly contradicts the need for regions of high salinity which remain stationary to support ridge growth over extended periods of time.

In the following section I will introduce a feedback mechanism between surface and subsurface dynamics which is able to pin the roots of the downwelling plumes in space long enough for patterns to grow on the surface. Since evaporation at the surface is driving the system and it is the system parameter which can most readily be modified by outside influence, I propose that the feedback mechanism acts as modulation on the evaporation rate $E(X)$, which is then varying spatially. One can imagine that the presence of a salt crust of varying thickness and porosity can modulate the evaporation through the surface. To identify, how the evaporation rate could be modulated, I relate this idea to research about evaporation from a porous medium. Among the mechanisms which have been identified to influence evaporation are $[174,175]$ :

- ambient conditions, e.g. temperature and humidity of the air over the porous medium,

- the type of the diffusing ion and its concentration and

- the presence, morphology and homogeneous or heterogeneous distribution of a salt crust.

From the concentration profiles measured in the field (see Sec. 4.4.2) I know that downwellings are aligned with ridges and suspect that the presence of a ridge locally reduces evaporation rate. While it is beyond the scope of this work to explore the exact mechanism of this modulation, in the following I will show how the underground dynamics behave if evaporation at the surface is not uniform anymore. At the end of this section I will also present some circumstantial evidence from the field, illustrating the ability of ridges to locally influence conditions which impact the evaporation rate.

\subsubsection{Non-uniform boundary condition}

As described in Sec. 3.1.7, I apply a sinusoidal modulation to the vertical velocity component in the simulations: $U_{Z}(X, 0)=U_{Z}^{0}-A_{\text {mod }} \cdot \cos \left(a_{\bmod } X\right)$. In Fig. $4.24 \mathrm{I}$ show spacetime diagrams of simulations at $\mathrm{Ra}=100$ with modulation wavenumbers of $a_{\text {mod }} \in\{0.79,1.59,2.36,3.14\} L$ and $A_{\text {mod }}=0.4$. It is apparent that the downwellings are initiated at the locations of the minima in the modulation. Roots of the downwellings stay aligned with the minima for some time $T_{A}$ and then start to fluctuate and coalesce into larger plumes again. The alignment time $T_{A}$ is defined 
as the time period the number of plumes is equal to the number of minima in the evaporation rate imposed at the top boundary (see Sec. 3.1.8 for details).

The length of $T_{A}$ depends on the modulation wavenumber $a_{\text {mod }}$. Even after the alignment breaks down, the origination and coalescence of small plumes and position of large plumes seem to be more regular and ordered as in a system with uniform flow at the top boundary. In the following I will quantify the alignment time $T_{A}$ and its dependence on the modulation amplitude $A_{\text {mod }}$ and wavenumber $a_{\text {mod }}$.

\subsubsection{Pinning}

I scanned $T_{A}$ for amplitudes in the range $A_{\text {mod }}=0.01-1.0$ and wavenumbers in the range of $a_{\text {mod }}=0.31-3.76$ and $\mathrm{Ra} \in[80,100,140,200]$. Results for $\mathrm{Ra}=100$ and a selection of amplitudes are shown in Fig. 4.25. The wavenumber of the most unstable mode $a_{\Omega} \approx$ 3.01 is indicated as dotted gray line.

It is apparent that $T_{A}$ increases as the strength of the modulation $A_{\text {mod }}$ increases. The alignment time has a pronounced maximum around $a_{\text {mod }}=1$, which shifts slightly towards higher wavenumbers for increasing amplitudes but is far smaller than the wavenumber of the most unstable mode $a_{\Omega}$ for $\mathrm{Ra}=100$. Most notably, $\mathrm{T}_{A} \geq 2 \mathrm{~T}$, even for very low modulation amplitudes $A_{\text {mod }}=0.01$ and $A_{\text {mod }}=$

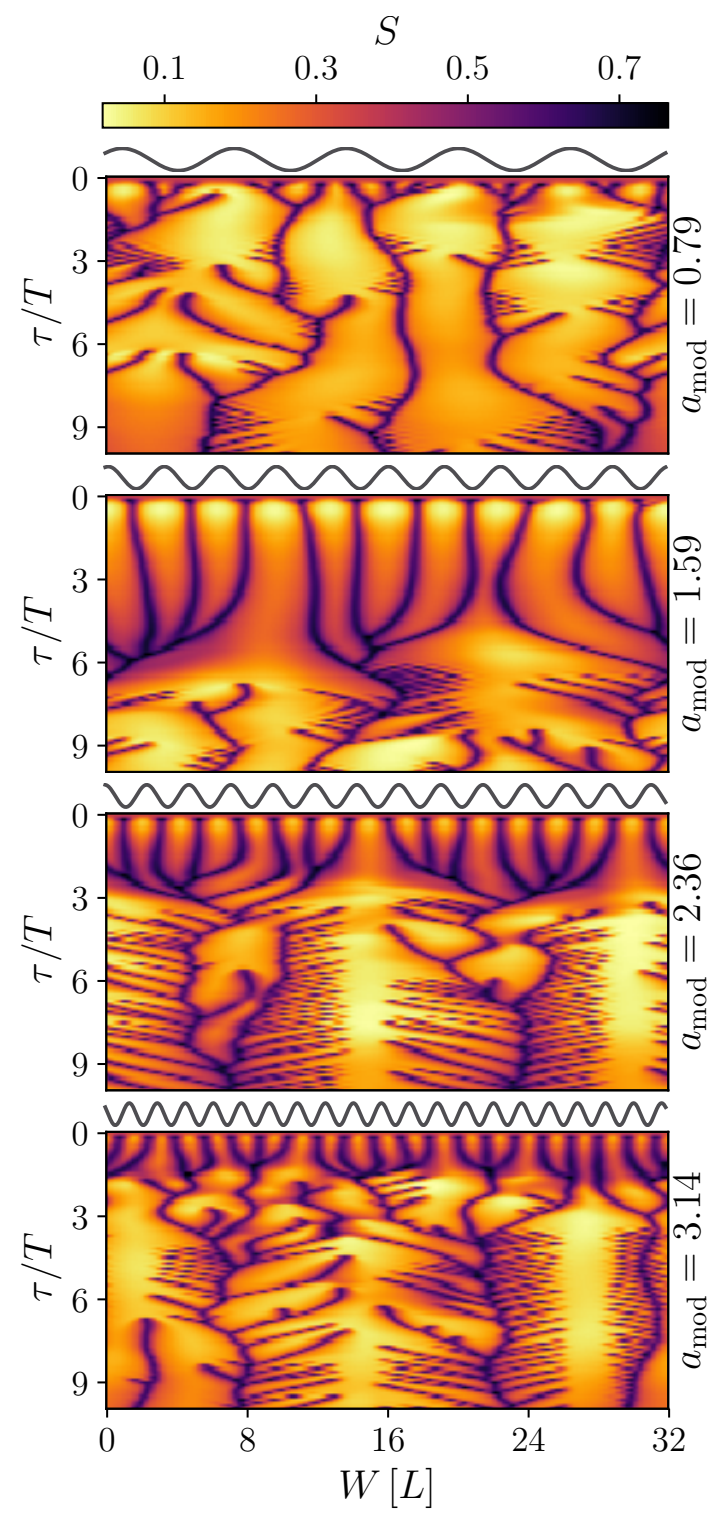

FIGURE 4.24: Salinity spacetime diagrams for simulations with nonuniform boundary $U_{Z}(X, Z=0)$ at $\mathrm{Ra}=100, A_{\mathrm{mod}}=0.4$ for different modulation wavenumbers.

Positions of the minima and maxima in the modulation are indicated above the diagrams in gray.

0.1 . This corresponds to about 1300 days in nature with $T=D / E^{2}$, using the modified diffusion coefficient of $D=1.25 \cdot 10^{-9} \mathrm{~m} / \mathrm{s}$ (as discussed in Sec. 4.3.4) and the evaporation rate of $E=0.4 \pm 0.1 \mathrm{~mm} /$ day measured by [44,37] at Owens Lake (see Sec. 3.3.4 for details). This would pin the roots of the downwellings long enough to enable ridges to grow to a significant height - given that erosion by wind is smaller than the growth rate.

In Fig. 4.26 I show a colormap of alignment times for a larger selection of amplitudes. Alignment times have a rather sharp peak in the range of $a_{\text {mod }}=0.6-1.3$ 
- the same wavenumber range where most of the wavenumbers measured in the field tended to cluster in. For larger amplitudes I also find significant alignment at larger wavenumbers. In the field, a modulation amplitude of $A_{\mathrm{mod}}=1$ would correspond to an evaporation rate of $E=0$ below ridges, which does not seem feasible. A reduction of evaporation by $1-10 \%$ however seems plausible.

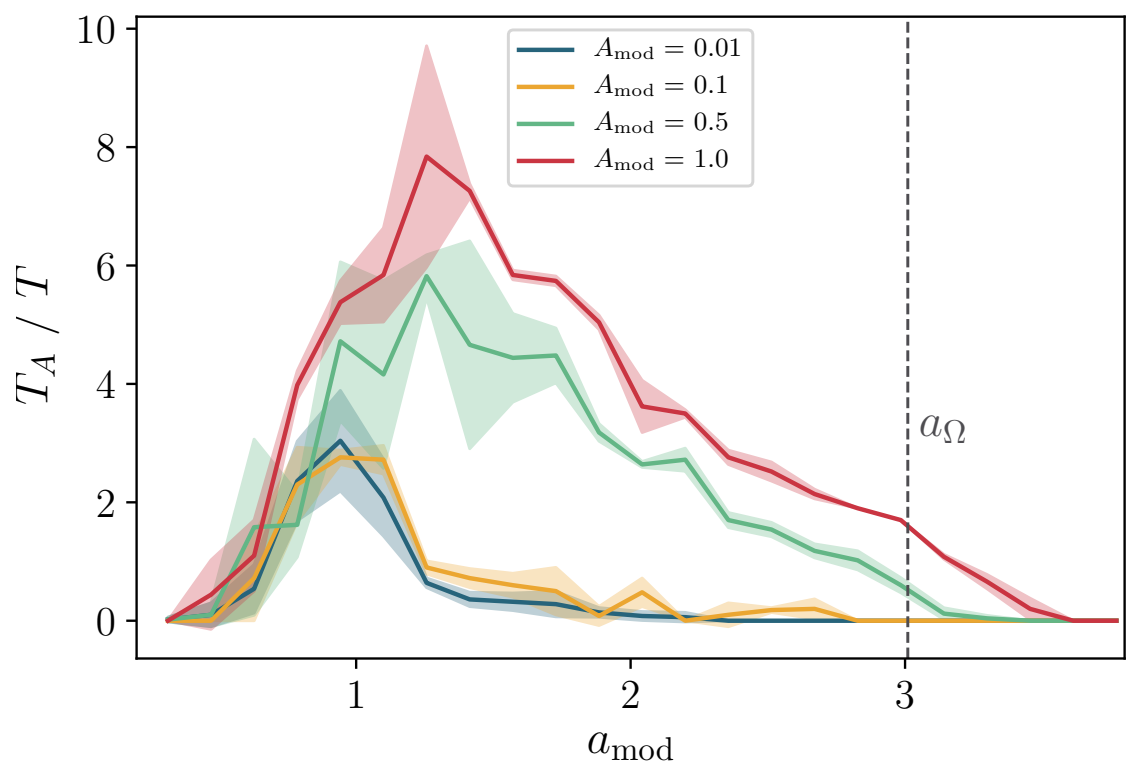

FIGURE 4.25: Alignment times $T_{A}$ over modulation wavenumbers $a_{\text {mod }}$ for simulations at $\mathrm{Ra}=100$, amplitudes $A_{\bmod } \in\{0.01,0.1,0.5,1.0\}$ and wavenumbers $a_{\text {mod }}=0.3-3.76$. Measurements are averages over ensembles of five simulations each, uncertainties are standard deviations. The wavenumber of the most unstable mode $a_{\Omega}(\mathrm{Ra}=100)$ is indicated as dotted gray line.

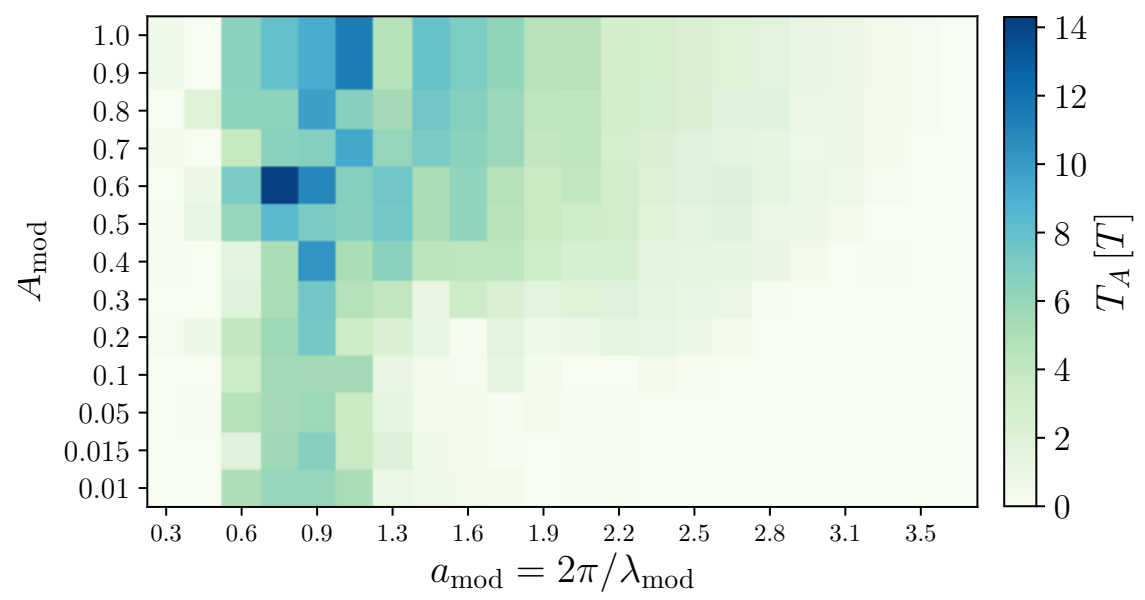

FIGURE 4.26: Alignment times $T_{A}$ over modulation wavenumbers $a_{\bmod }$ for simulations at $\mathrm{Ra}=100$, amplitudes $A_{\mathrm{mod}}=0.01-1.0$ and wavenumbers $a_{\text {mod }}=0.31-3.76 . T_{A}$ from short alignment (light green) to long alignment (dark blue). Data points are $T_{A}$ measurements for single runs for each set of $\left(A_{\text {mod }}, a_{\text {mod }}\right)$. Note that the modulation amplitudes $A_{\text {mod }}$ on the $y$-axis do not follow a linear scale. 


\subsubsection{Feedback in the field}

As determined by [174], the presence of a salt crust has next to no influence on the evaporation from the porous medium, since it is porous and wettable in itself and will conduct water to the surface similar to the porous medium. Ambient conditions and activity of the saline solution were determined to influence evaporation rate to a large degree through their influence on vapor pressure. Additionally, temperature also influences to which degree the evaporation rate will be reduced by an increased salt concentration $[174,37]$.

Temperature and humidity are possible parameters that can be influenced by the presence of ridges in the field, since they could shield the soil from direct solar radiation and hamper air circulation and therefore transport of vapor. To investigate the influence of ridges on temperature and humidity, measurements of these observables from within ridges and a direct comparison to measurements from the polygon center were deemed necessary.

In my first field study in late 2016 I recorded the temperature in the center of a single polygon as well as below a ridge over a week. Additionally I recorded the humidity in a ridge at two different sites. Unfortunately I do not have the direct comparison of center to ridge from a single site for the humidity. Nevertheless I can at least estimate the magnitude of the temperature change and find some indication for its modulation by the presence of a salt ridge.

In Fig. 4.27 (a) I show the temperature development over six days in late 2016 inside a salt ridge (dark red) and at a polygon center (light red) at Owens Lake, site T29-3 and the temperature difference (blue). Diurnal fluctuations of temperature between about $10^{\circ} \mathrm{C}$ at 2:00 $\mathrm{PM}$ and about $0{ }^{\circ} \mathrm{C}$ at 6:00 $\mathrm{AM}$ are clearly visible. Maximum temperatures during the day are similar between center and ridge. Temperatures inside the ridge however seem to drop faster and lower at night. The temperature difference is about $2{ }^{\circ} \mathrm{C}$. A decreased temperature would most likely also decrease the evaporation rate. This agrees with the results from my investigation of plume alignment with minima in the evaporation rate (see Fig. 4.24). However, these results have to be viewed with caution as the measurements were taken in winter. Temperatures at Owens Lake in summer are significantly higher (about $30-40^{\circ} \mathrm{C}$ at noon) and, as described in Sec. 4.7.2, polygon growth is possibly significantly faster during the hot season.

The melting temperature of Mirabilite into anhydrous sodium sulfate (Thenardite) and water starting at $8^{\circ} \mathrm{C}$ for a mixture of $8 \mathrm{wt} . \%$ Mirabilite and water is indicated as gray area (see Sec. 5.4.3 for details on Mirabilite melting). During the six days measurement period, temperatures at noon entered the region were Mirabilite melting is possible.

Figure 4.27 (b) shows the development of the relative humidity over six days in late 2016 inside a salt ridge at site T29-3 (red) and at a polygon center at site T36-3 (blue). The relative humidity measured inside the ridge is equal (on the first, third and sixth day) or by up to higher $15 \%$ higher (on the second, fourth and fifth day). 

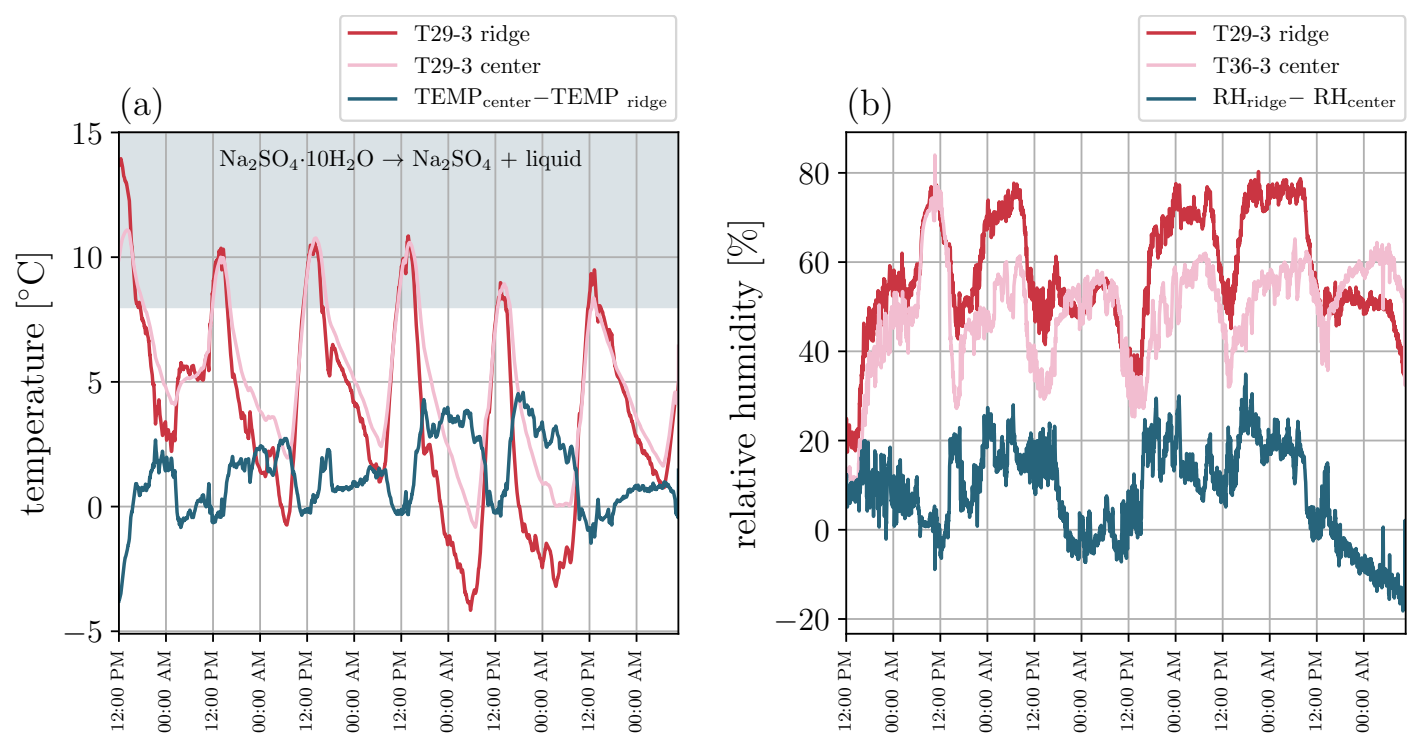

FIGURE 4.27: (a) Temperature recorded from two sensors placed inside a ridge (dark red) and in the middle of a polygon (light red) at Owens Lake, site T29-3 as well as temperature difference $\mathrm{TEMP}_{\text {center }}-\mathrm{TEMP}_{\text {ridge }}($ blue) and melting region of Mirabilite (gray area). (b) Relative humidity recorded below a ridge at site T29-3 and in the middle of a polygon at site T36-3 and relative humidity difference $\mathrm{RH}_{\text {center }}-\mathrm{RH}_{\text {ridge }}$ (blue). Measurements were taken every two minutes in a time period of six days between November 26th and December 2nd, 2016.

Humidity differences are most pronounced during nights and mornings but are are preserved to some extent over the course of the day. The two sites had a distance of approximately two kilometers, therefore I do not expect regionally different weather conditions. Nevertheless, since the measurements were not taken in close spatial proximity, my ability to draw reliable conclusions about the capacity of ridges to modulate the relative humidity above the soil surface remains very limited.

Nevertheless, both the temperature as well as the humidity measurements give an indication that the presence of a ridge is able to influence ambient factors such as temperature and relative humidity above the soil to a significant degree.

\subsubsection{Summary}

In my simulations I found that the roots of downwellings fluctuate in space as the system ages. To drive the growth of stationary ridges, underground dynamics need to stay aligned with the ridges on the surface. I investigated how feedback between the surface and underground dynamics affect the alignment of the downwelling plumes by modulating the evaporation rate at the surface. I find that even very low modulation amplitudes of $1-10 \%$ are sufficient to pin the downwellings for a time that corresponds to 1300 days in the field. Moreover for low modulation amplitudes only a small range of modulation wavenumbers, which are on the low end of the range of wavenumbers measured in the field, cause significantly long alignment. I also presented some evidence from temperature measurements in the field that reports a temperature difference at night of about $2{ }^{\circ} \mathrm{C}$ between polygon ridges and centers, with ridges showing a lower temperature. 


\subsection{Ridge growth}

While my primary interest in this work is the wavelength expressed by the polygonal patterns, their height $h$ is also an interesting observable. Ridge heights can be easily calculated based on the TLS scans of the surface relief and are listed for all field sites in Tab. 4.7. $90^{\text {th }}$ percentile values are in the range $h=2-15 \mathrm{~cm}$ while individual ridges reached heights of up to $30 \mathrm{~cm}$. It is a rather obvious conclusion that ridge height is an indicator for the age of a polygon, since ridges grow slowly as time passes and salt precipitates. As shown in Fig. 4.22, ridge height also correlates with polygon wavelength.

In the following I will present direct and indirect evidence of ridge growth as well as an estimate of growth rates. This will independently lead to a rough age estimate of the polygons which is consistent with the age estimate based on polygon wavelength and coarsening presented above.

\subsubsection{Indirect evidence for ridge growth}

At the tourist site at Badwater Basin about $500 \mathrm{~m}$ north of the sampling sites, the age of ridges was discernible based on ridge color. Older ridges were taller, wider and looked more weathered and brown, because of fine sand accumulating on them. Younger ridges were smaller, thinner and appeared in pristine white. Based on the knowledge that the site I saw was flooded about one year prior to my visit, I suggest that the tall brown ridges were not completely dissolved by the water during the flood but nevertheless covered by sediments carried with the water at the time of the flood. They could also have been covered by sand carried over the salt flats by the wind over time.
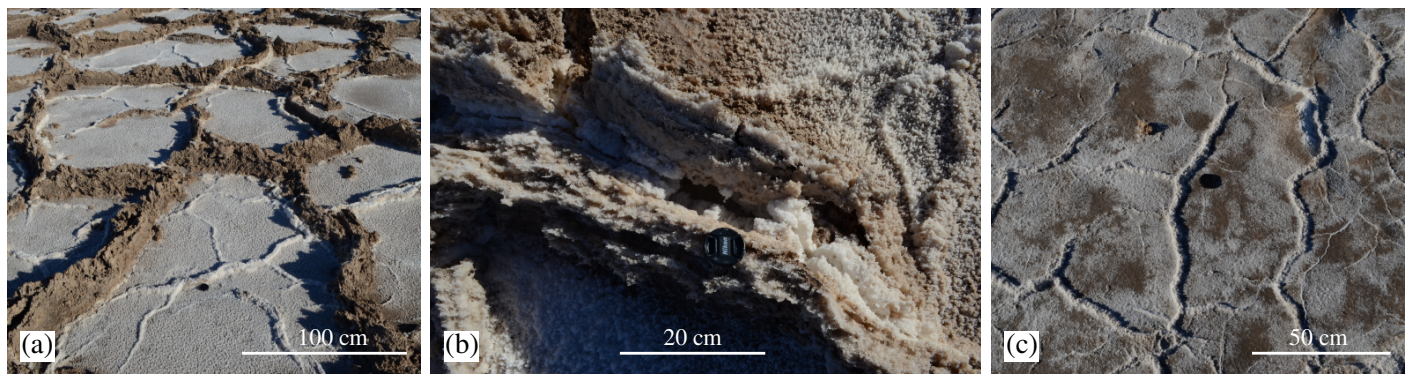

FIGURE 4.28: Images of salt polygons at Badwater Basin in winter 2016, about a year after a flood submerged the polygons. The black camera cap used as scale bar is about $5.5 \mathrm{~cm}$ in diameter. (a) Brown ridges are older (potentially from before the flooding of the previous year) and covered in sand. Ridges grow both from the inside of old ridges outwards (b) as well as in between older ridges (c).

Figure 4.28 (b) shows an old ridge, where salt crystals precipitated from the inside out and a gradient from white to brown is visible. Figure 4.28 (a) shows older (brown) polygons with a much larger length scale $(\lambda \approx 1 \mathrm{~m})$ than the younger, white ridges in their centers. Figure 4.28 (c) shows an area were only young ridges were present, which form a pattern with a length scale of $\lambda \approx 30 \mathrm{~cm}$, significantly smaller 
than the dominant pattern length scale of the old ridges. This could mean that polygons with a smaller length scale appear first and with time the dynamics in the underground coarsen and a larger length scale is selected. The flooding event maybe additionally selected for the tallest and therefore probably oldest ridges, if the water was not high enough to cover the ridges completely. Sadly, I was neither allowed to conduct a laser scan at the tourist site nor to collect samples, therefore my evidence for coarsening is based solely on visual inspection and remains circumstantial.

\subsubsection{Direct evidence for ridge growth}

During my first field campaign I recorded the overnight change at two sites by scanning the crust twice, once in the afternoon and again in the morning of the following day, after about 12 hours. Figure 4.29 shows a positive change of up to $0.6 \mathrm{~mm}$ in some places and a negative change of up to $-0.6 \mathrm{~mm}$ in other places. The overall change is neutral (Fig. 4.29 (a)) or positive (Fig. 4.29 (b)). The changes seem to occur predominantly at or around ridges and I therefore conclude that ridges are the most active part of the salt crust.
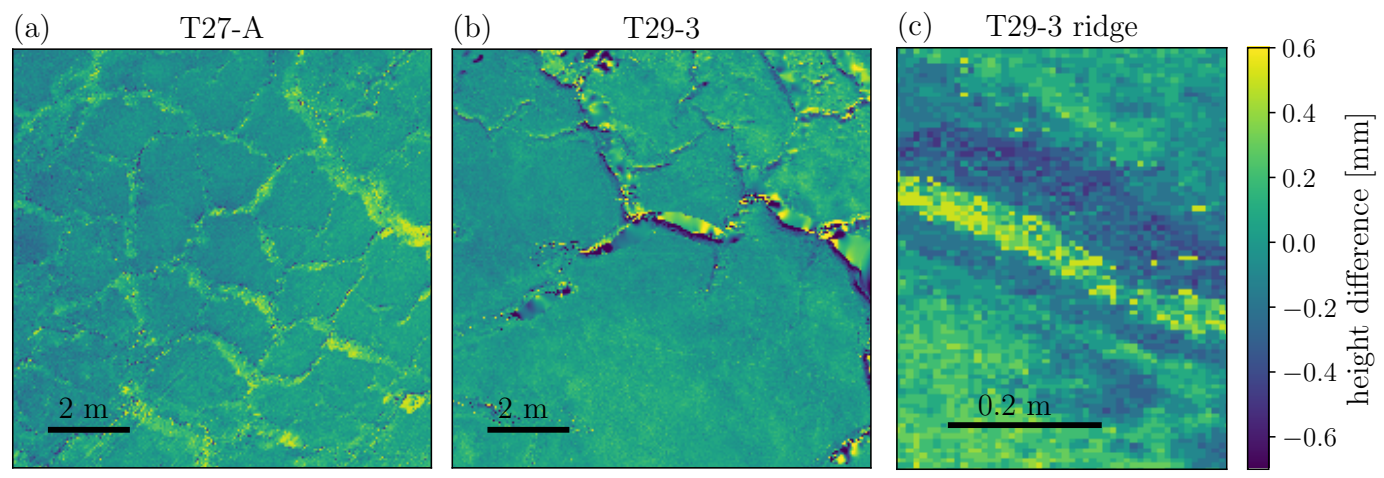

FIGURE 4.29: Overnight change from November 25th - 26th 2018. TLS scans were normalized by their mean value. (a) $11 \mathrm{~m} \times 11 \mathrm{~m}$ section of crust at cell T27-A, (b) $11 \mathrm{~m} \times 11 \mathrm{~m}$ section of crust at cell T29-3, (c) $0.4 \mathrm{~m} \times 0.8 \mathrm{~m}$ containing a single ridge from cell T29-3.

In Fig. 4.29 (c) I show the change along a single ridge: the ridge center seems to grow the most while the ridge sides seem to push inwards towards the center of the ridge. While this is only data for one night and two sites, it might shed some light on the mechanics of ridge growth itself. I conclude that ridge growth in winter is about $1 \mathrm{~mm} /$ day. This is a bit higher but comparable to the values of $0.44 \pm 0.41 \mathrm{~mm} /$ day and $0.23 \pm 0.14 \mathrm{~mm} /$ day reported by [8] for ridge growth in the Makgadikgadi Pans of Botswana in early August and from mid August to mid September respectively.

As described in Appendix B, the crusts at Owens Lake contain significant amounts of Thenardite. Since Thenardite turns into Mirabilite by integrating significant amounts of $\mathrm{H}_{2} \mathrm{O}$ into its crystal structure at low temperatures (during nights), and therefore increases its volume, height changes measured with the overnight scan might also be due to swelling of the crust at night, which was also observed by [8] in a similar field setting in the Makgadikgadi Pans of Botswana. 


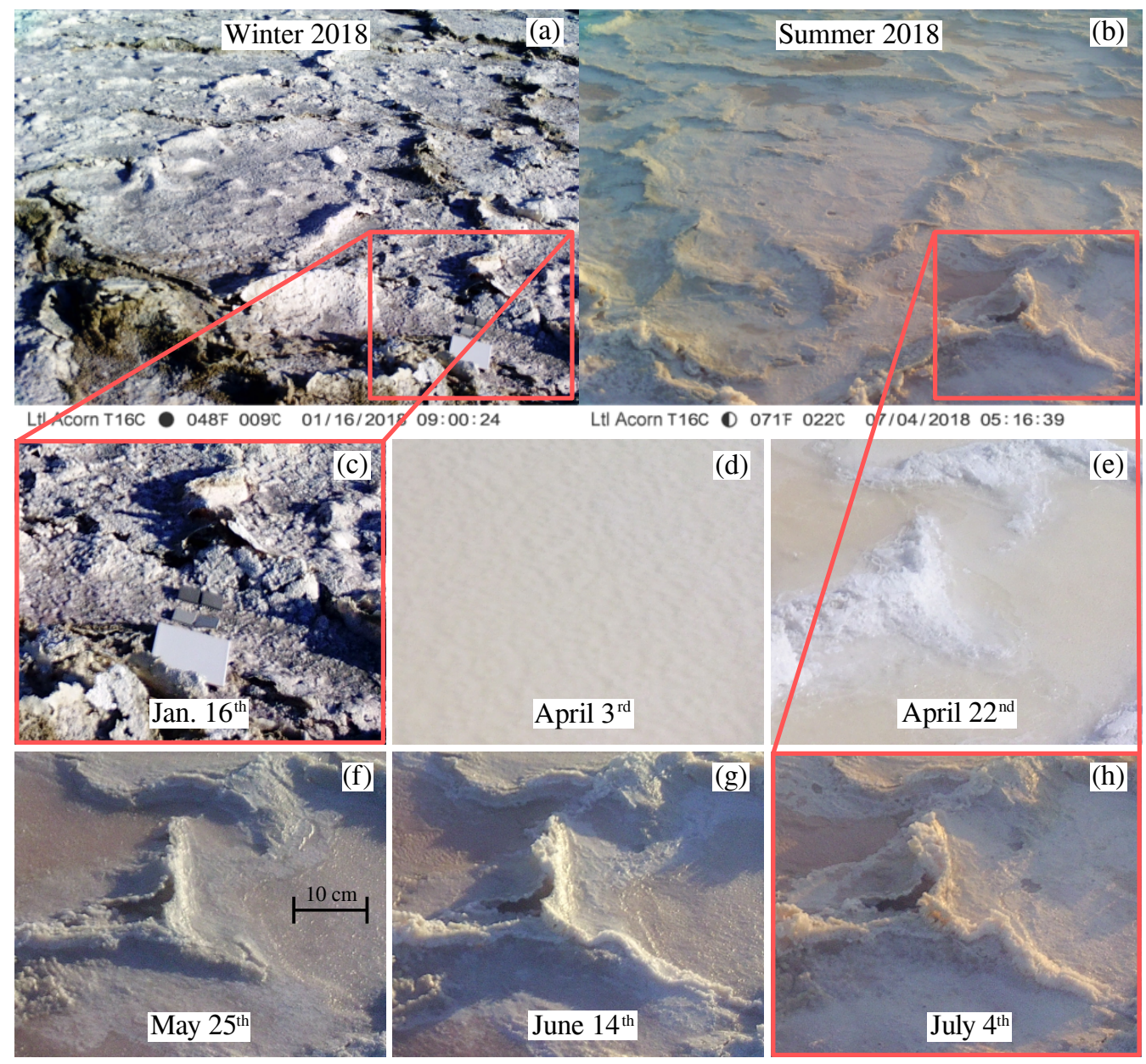

FIGURE 4.30: (a) and (b): Site T16 in January and July 2018. (c), (d) and (e): Zoom into bottom left field of view of the camera. (c) crust before flooding, (d) crust completely flooded, (e) ridge emerging from the water. (f), (g) and (h): Evidence for the growth of a polygon ridge by about $5 \mathrm{~cm}$ over the course of 20 days from May $25^{\text {th }}$ to July $4^{\text {th }} 2018$.

As described in Sec. 4.6.3 and shown in Fig. 4.27, temperatures at the field sites during the time of my visit were in the range were melting of Mirabilite is possible. It is unclear, whether the swelling leads to a permanently increased volume of the crust or if the ridges shrink again after the water molecules are expelled.

During my second field campaign I installed three cameras at cells T16, T10-3 and T2-5, as described in Sec. 3.3.2. The cameras recorded images for about half a year. Site T10-3 was flooded shortly after I left and did not dry until July. The camera at site T2-5 failed after about a month and did not record significant changes during the time it was operational. Site T16 was also flooded on January $20^{\text {th }}$ but dried in the end of April. No significant growth was visible in the 4 days before initial flooding.

In Fig. 4.30 (a) and (b) I show the crust in the field of view over the course of 6 months and after a flooding incident. Panel (c) shows a zoom into the bottom right part of (a) and contains white and gray tiles that were initially placed to provide a white balance and scale bar. Panels (d) and (e) shows the same magnified section in 
the beginning and end of April, completely flooded and mostly dried respectively. In panels (f) through (h) I show the magnified section at May $25^{\text {th }}$, June $14^{\text {th }}$ and July $4^{\text {th }}$. The crust is still humid but no standing brine is present anymore. The ridge shows significant vertical growth of about $3-5 \mathrm{~cm}$ over the course of 20 days. This corresponds to a growth of approximately $2 \mathrm{~mm} /$ day. This is consistent with the growth rate of $1 \mathrm{~mm} /$ day during a period of 12 hours measured via TLS scans in winter.

The increased growth rate can be readily explained by the higher temperatures and therefore increased evaporation during spring and summer. While the camera's temperature sensor is not very accurate, it still provides an estimate for the average temperatures during winter and summer: During winter the camera consistently recorded temperatures around $0{ }^{\circ} \mathrm{C}$ during the day, whereas during summer temperatures around $45^{\circ} \mathrm{C}$ were common. As expected for a desert climate, the temperature difference is substantial.

Fig. 4.31 (a) shows ridge positions (indicated in red) in the camera's field of view before the flooding while panel (b) shows ridge positions after the flooding (indicated in blue). The overlay of the two, shown in panel (c), indicates that ridges that grew after the flooding are somewhat but not completely aligned with the positions of ridges before the flooding.

\subsubsection{Summary}

As already reported by [8], the crusts in salt pans are very dynamic and grow significantly over
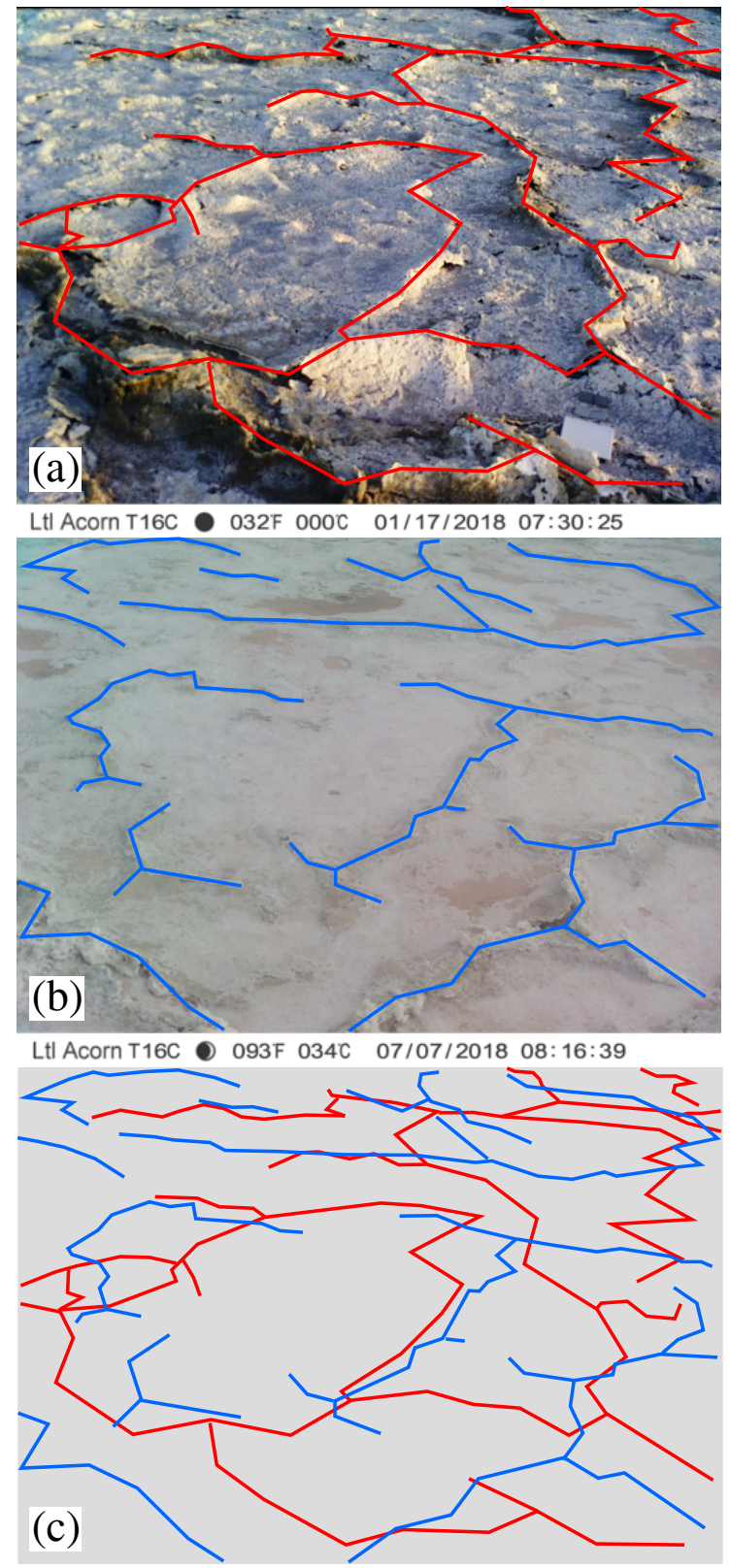

FIGURE 4.31: Images of a $2 \times 4$ section of salt crust at Owens Lake at (a) January $17^{\text {th }}$ and (b) July $7^{\text {th }}$. (c) Indication of ridge location before (red) and after (blue) flooding. 
the course of several weeks. Evi-

dence from repeated TLS scans and timelapse imaging of crusts at Owens Lake indicates that crusts grow with a rate of about $1-2 \mathrm{~mm} /$ day. Based on these measurements I would predict a ridge growth of about $30-60 \mathrm{~cm}$ over the course of a year. The maximum height of ridges I encountered at Owens Lake, Badwater Basin and Bristol Playa was about $30 \mathrm{~cm}$, measured with a folding rule. This is larger than the values in Tab. 4.7 , since these values only reflect $90^{\text {th }}$ percentile values. In Sec. 4.5.4 I estimated the age of the polygons to be around one year based on their coarsened wavelength and recorded flooding events. This seems to be consistent with my very rough approximation of ridge growth rate based on timelapse photography and overnight TLS scans, since I also expect significant influence of weathering caused by the strong winds in the area.

TLS scans as well as timelapse images and images from ridges colored by sand at Badwater Basin all indicate that crust growth is most active along ridges, and that the ridges are pushed up by the surrounding crust as they grow. This leads me to the conclusion that while salt precipitation is increased at the center of the ridges because of high salinity, the crust surrounding the ridges also continues to grow and exerts horizontal pressure on the ridges.

\subsection{Conclusion}

In the above chapter I was able to find evidence for the predictions I made in Sec. 1.4 about the advection-diffusion dynamics and its connection to the polygonal salt ridge patterns visible in salt deserts. In summary, these predictions and the corresponding evidence are:

Instability: I determined the critical Rayleigh number for the onset of instability by doing a linear stability analysis of the equations. I found two slightly different critical Rayleigh numbers for the "salt pan" (constant flow rate) and the "salt lake" (constant pressure) boundary conditions at the soil surface. I measured the parameters necessary to calculate the Rayleigh number in the field and determined, that all but one field sites show a value of $\mathrm{Ra}>\mathrm{Ra}_{c}$ and that the median Rayleigh number for Owens Lake, where I conducted most of my research, is $\mathrm{Ra}=237$. I conducted experiments in setups with Rayleigh numbers in the stable and unstable regime. As predicted, I found convective dynamics only in the setups where $\mathrm{Ra}>\mathrm{Ra}_{c}$.

Density stratification: If the system is driven by evaporation, a vertical salinity gradient will built up below the soil surface. I investigated the buildup of this gradient in the numerical simulations and characterized the length scale $L^{\prime}$ of the salinity gradient for systems at different Rayleigh numbers. I found evidence for vertical salinity gradients both in the analogue Hele-Shaw experiments as well as in nine of the field sites I investigated. 
Convection and co-location: If both a vertical density stratification is present and the system Rayleigh number is above the critical value, the systems become prone to a buoyancy driven convective instability. This leads to downwelling plumes of high salinity and therefore horizontal salinity gradients. I found downwelling plumes both in the experiment and the field setting. In the experiment the salinity within the plumes is increased over the background salinity by a factor of approximately two. In the field, the salinity is increased by a factor of 2-4 over the background salinity. In the field, the downwelling plumes of high salinity are co-located with the ridges emerging from the crust. This is a very strong indication that the buoyancy driven convective dynamics in the underground are directly linked to the patterns visible at the surface.

Pattern wavelength: The linear stability analysis allows for a prediction of the dependence of the wavenumber of the most unstable mode on the Rayleigh number. To investigate this scaling, I implemented numerical simulations of the advectiondiffusion dynamics and investigated the development of the wavenumber of the convective dynamics with Rayleigh number and relative age of the systems. While at the beginning simulations follow the predicted dependence of $a(\mathrm{Ra})$, I found significant coarsening behavior in older systems. The coarsened systems always tend to a range of wavenumbers between $a=0.2$ and $a=2.5$. I visualized the flows in analogue experiments and measured the wavelength of the convective dynamics and also found a weakened dependence of the wavenumber on the Rayleigh number. The experiments correspond to systems with a very high relative age and tended towards the low end of the wavenumber spectrum whereas the patterns observed in the field correspond to medium-aged systems and tend towards the middle of the wavenumber spectrum of $a=0.5-2.5$.

Feedback: The numerical simulations of the advection-diffusion dynamics show that the downwelling plumes are not stationary. This is an apparent contradiction with the need for stationary regions of high salinity to support pattern growth at the surface for an extended period of time. I therefore introduced a feedback mechanism between crust and underground dynamics which is able to pin the downwellings below the ridges. I proposed a feedback mechanism in the form of a modulation of the evaporation rate. Numerical simulations of systems with such a modulation showed pinning of the dynamics for long enough to support ridge growth in nature. In addition, the pinning also prefers a wavenumber in the same range as the coarsened dynamics and the wavenumbers measured in the field tend to. Last but not least I was able to find some evidence in the field, indicating an impact of ridges on local temperature and humidity, which could in turn be enough to lower the evaporation rate below ridges and pin the dynamics. 
Ride growth timescale: In addition to the evidence supporting my predictions, I presented direct evidence from the field, demonstrating the growth of ridges over the course of a day and several weeks. Growth rates calculated from terrestrial laser scans and time-lapse photography indicate, that ridges grow between 1-2 mm/day, leading to an estimation of ridge heights in the range of $30-60 \mathrm{~cm}$ after one year of growth, if erosion by wind is neglected. This is consistent with the maximum ridge height found in the field. Moreover, I found a clear correlation between ridge height and pattern wavelength- This demonstrates once again that the dynamics of pattern growth occur on rather short timescales compared to other geomorphological processes and that the polygonal ridge patterns are dynamical themselves. 



\section{Chapter 5}

\section{Discussion}

In this chapter I will discuss the reliability of my results under aspects of the approximations I made as well as the uncertainty of the various measurements. Additionally, I will discuss the importance of my results given the current state of literature in this field of research. As in Chapter 3, this chapter follows the structure and order of methods I used to investigate the phenomenon: theoretical considerations, numerical simulations, experiments and field research.

\subsection{Approximations}

In Sec. 1.3.1 I motivated many of the assumptions and simplifications underlying my model of the advection-diffusion dynamics of flows in a porous medium. Nevertheless, two simplifications warrant a more detailed discussion, since the assumptions which underly the simplifications break down for some parts of my work. In the following I will first discuss the impact of dispersion on diffusivity in connection with fluid velocities in the experiment and field. Secondly I will revisit the impact the dependence of the kinematic viscosity on fluid density has on the evolving dynamical process.

\subsubsection{Diffusion and Dispersion}

The diffusion tensor $\boldsymbol{D}$ contains the coefficients for diffusion along the dimensions of fluid flow. According to Taylor dispersion, diffusion can be enhanced by shear flows in the direction of fluid flow [176, 177]. In Sec. 2.3 I briefly mentioned that for my model of the advection-diffusion system, I choose to neglect dispersion. In the following I will estimate the contribution of dispersion to both the diffusivity in the experiment as well as the field setting, given the velocities measured in the system and argue, why it is still warranted to neglect dispersion in my model of the system.

The effective diffusion coefficient $D_{L}$ in the longitudinal direction of fluid flow in a porous medium, including a contribution from the molecular diffusion coefficient $D_{M}$ (discussed in Sec. 4.3.4) is modeled as $[58,178]$

$$
D_{L}=D_{M}\left[1+\frac{2}{105}\left(\frac{\mathrm{Pe}}{2}\right)^{2}\right] ; \quad \mathrm{Pe}=\frac{\langle u\rangle b}{D_{M}}
$$


Here Pe is the Péclet number, which describes the ratio of advective to diffusive transport in the fluid and $b$ is the spacing between the walls of a Hele-Shaw cell, which describes the length scale of the porous medium. Using $b$ is only warranted, if the Hele-Shaw setup is used to mimic a porous medium. In the case of my analogue experiments, the Hele-Shaw cells were filled with sand, and therefore the length scale of the porous medium is better described by the characteristic length scale of the sand. I use the Sauter diameter of the porous medium, as described in Sec. 3.2.2, which is $d_{S} \approx 150 \mu \mathrm{m}$ in the experiments, to calculate the Péclet number.

For Péclet numbers $\mathrm{Pe}<1$, dispersion is negligible compared to diffusive transport in the system. Péclet numbers calculated following Eq. (5.1) are listed in Tab. 5.1. Even for the highest evaporation rates in the experiment, Péclet numbers are of order $\mathcal{O}\left(10^{-1}\right)$, with Péclet numbers for the field setting being much lower and of order $\mathcal{O}\left(10^{-4}\right)$. I conclude that neglecting dispersion in the modeling of the dynamics is warranted.

\begin{tabular}{ccc} 
setting & $E 10^{-9}[\mathrm{~m} / \mathrm{s}]$ & Pe \\
\hline \multicolumn{3}{c}{ Experiment } \\
\hline$u_{\min }$ & $34 \pm 4$ & $(7 \pm 1) \cdot 10^{-3}$ \\
$u_{\text {avg }}$ & $362 \pm 260$ & $(8 \pm 6) \cdot 10^{-2}$ \\
$u_{\max }$ & $1172 \pm 75$ & $(2.5 \pm 0.2) \cdot 10^{-1}$ \\
\hline \multicolumn{3}{c}{ Field } \\
\hline Owens Lake & $4.63 \pm 1.16$ & $(2.8 \pm 2.9) \cdot 10^{-4}$ \\
Badwater Basin & $2.08 \pm 1.16$ & $(2.2 \pm 0.4) \cdot 10^{-4}$ \\
\hline
\end{tabular}

TABLE 5.1: Evaporation rate and Péclet number calculated following Eq. (5.1) for the minimum, average and maximum velocity in the experiment as well as Owens Lake and Badwater basin.

\subsubsection{Dynamic viscosity and evaporation rate}

As mentioned in Sec. 2.3, I chose to neglect the influence of density on the fluid's viscosity. The viscosity increases by a factor of about two between fresh water and concentrated brine [109]. Nevertheless, the activity of the fluid and therefore its evaporation rate also decreases as salinity increases [157, 179]. Since both the evaporation rate and the viscosity enter the Rayleigh number in the denominator ( $\mathrm{Ra}$ $=\kappa \Delta \rho g(E \mu \phi)$ and their respective changes with salinity are comparable, I expect the two effects to mutually cancel when only their effect on Ra is concerned. However, one has to keep in mind that for other effects such as planform selection of the fluid dynamics (see Sec. 5.5 for a discussion) and the feedback at the surface, both dependencies might have a considerable effect.

I can conclude that the assumption of constant viscosity and negligible dispersion is warranted for the scope of my work but need to be revisited for a more detailed experimental quantification of the dynamics as well as modelling of planform selection. 


\subsection{Simulation}

There is a rich selection of literature on the topic of porous media flow, a good review of which was compiled by [67] and [73]. The aim of the simulations conducted within the scope of this work was not to improve on the accuracy or extent of these simulations but to use them to inform the experimental and field research as well as to investigate the boundary conditions specific to the salt playa problem. In the following I will detail where our simulations are similar to existing approaches and were we implemented and investigated new ideas. Last but not least I will explain the limitations of the simulations where the investigation of the pattern formation in salt playa phenomenon is concerned.

\subsubsection{Comparison to literature}

What distinguishes my system from similar systems described in the literature is my choice of the uniform flux boundary condition, which reflects the salt pan setting of constant evaporation found in the field (see Sec. 2.6) for details). The dynamics I observed in my simulations of the buoyancy driven convection in porous media show a rich set of behaviors (see Sections 4.3.2, 4.5.1 and 4.6.1). In the following, I compare the dynamics I observed in my implementation to the known dynamics reported from similar systems.

A very detailed description of the dynamics of the system for constant dissolution flux - the rate at which solute dissolves at the top boundary and enters the system is provided by [73]. In their simulations the top boundary is in one case impermeable to fluid and in the other case semi-permeable. These boundary conditions resemble the application of $\mathrm{CO}_{2}$ sequestration into a porous medium and are slightly different than the boundary conditions I use for my simulations (see Sec. 2.6.1). They describe a total of six regimes in the dynamics, which occur one after the other from start to "shutdown":

1) Diffusive growth of the high salinity boundary layer.

2) Onset of instability and linear growth of the initial plumes.

3) Vertical acceleration of the downward flowing high salinity plumes, drawing much of the accumulated salt at the boundary layer downwards. [73] calls this the flux growth regime.

4) Once long enough, the downward flowing plumes start interacting with each other and enter the merging regime, where the dynamics coarsen.

5) As result of the coarsening, salinity can accumulate again to a larger extent at the top boundary and small plumes form again, which move horizontally into the larger plumes. This regime is called the re-initiation regime. 
6) Once the first plumes impact the bottom boundary of the system, the simulation domain starts to saturate with salt and will shut down eventually.

In my simulations I observe regimes two through six, since I already start my simulations with the steady state solution for the salinity distribution and therefore a salinity gradient (see Sec. 3.1.7). In all simulation runs, all measurements are completed before the shutdown regime to avoid unphysical results. In Fig. 5.1 I show exemplary snapshots of a simulation in the different regimes (see Fig. 4.16 for an analysis of the wavenumber in the different regimes). Panel (a) shows the onset of instability where plumes with a small wavelength start to form at the top boundary. In panel (b) I show the flux regime, where plumes accelerate downwards but the small wavelength is still preserved. In panel (c), plumes have started to coarsen and merge whereas in panel (d), small plumes start to emerge at the top boundary again, as the system enters the re-initiation regime. Panel (e) shows the last snapshot of the simulation at $\tau=125 \tau_{S}$. Plumes have reached the bottom boundary and a substantial amount of salinity has accumulated in the system. Since there are still plumes of high salinity originating at the top boundary (and plumes of low salinity originating at the bottom), the shutdown regime has not been reached yet.
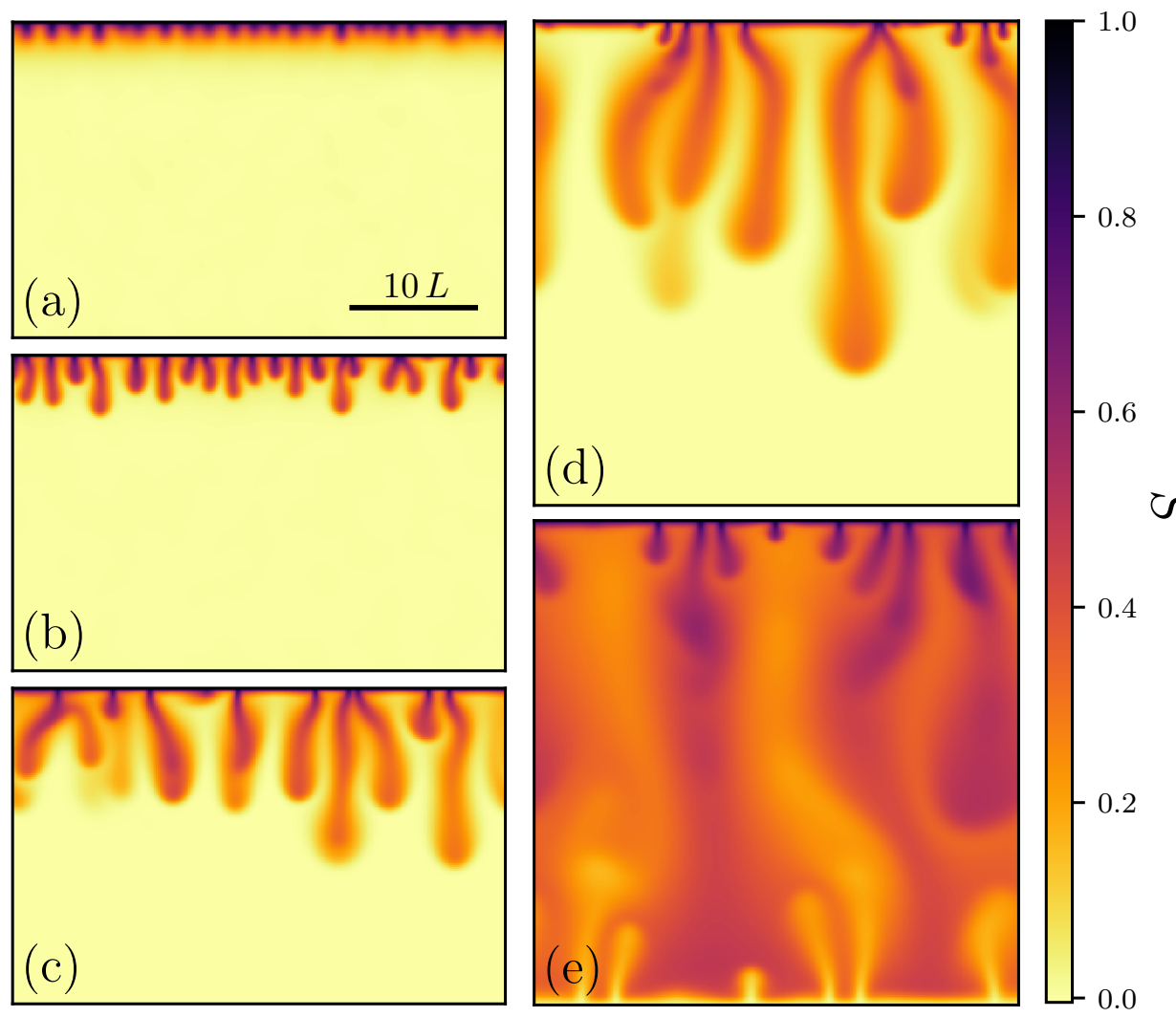

FIGURE 5.1: Illustration of the different regimes of the dynamics. The panels show the salinity field $S \in[0,1]$ with $S=0$ in yellow and $S=1$ in purple for a simulation at $\mathrm{Ra}=100$ with a system size of $40 \times 40 \mathrm{~L}$. (a) Onset of instability at $\tau=0.1 \tau_{S},(b)$ flux growth regime at $\tau=2 \tau_{S},(\mathrm{c})$ merging regime at $\tau=6.5 \tau_{S},(d)$ re-initiation regime at $\tau=15.5 \tau_{S}$ - note the small nascent plumes at the top boundary and (e) increasing saturation of the system at $\tau=125 \tau_{S}$. 
I quantified the linear growth regime, compared it to theoretical predictions for the dominant perturbative wavelength and reproduced the results known from literature $[58,180,181]$. The flux growth regime is of most interest for applications where flux of the dissolved quantity in the system is of importance, such as $\mathrm{CO}_{2}$ sequestration. Since this is not the case for my application to pattern formation in salt deserts, I did not quantify flux or the Nusselt number [182] or the system. I invested considerable effort to quantify the merging regime and characteristic time scale or age of the system, since it very likely is of great importance to the length scale and type of pattern which can be observed in nature. These results are, to my knowledge, new and might be of interest for other surface expressions where underground dynamics play a role. They might also allow for an inference about subsurface dynamics in places where only information from the surface is accessible. Finally I investigated the re-initiation regime by imposing a different, non-uniform boundary condition on the top boundary of the system. Onset of pinning and pinning times give an indication of the preferred length scale of the system dynamics and seem to resonate with the length scale which is selected in the field. So far I only tried sinusoidal modulations, but different kinds of modulations as well as non-periodic modulations and their impact on the dynamics could be an interesting target for further investigation.

\subsubsection{Limitations of the simulation}

The numerical approach and implementation is described in Sec. 3.1. Implementation of the simulation was done in Python. We used a sequential approach even though all of the computationally costly processing steps such as matrix multiplication and fast Fourier transforms can be parallelized on GPUs. This was due to lack of time for the implementation, since most of the work on the implementation was done by Marcel Ernst within the scope of his Master's thesis. As my project included significant contributions from both the field campaigns as well as the experiments, I did not prioritize further development of the simulations. As a result, the code was not streamlined and simulations of large system domains, high resolutions and very long run-times were challenging due to computational cost and simulation duration.

I chose the domain width and depth to roughly resemble the dimensions of the laboratory experiments, which was possible with the existing implementation. As with the experiments, boundary effects by the limited extent in horizontal direction can not be completely excluded. Boundary conditions are periodic but since in the coarsened state the domain only had space for 3-8 large downwelling plumes, a quantization of the emerging dynamics is unavoidable.

The limited depth of the system also limited my ability to investigate the coarsening dynamics at great depth. My main interest is in the dynamics near the surface, which can influence pattern formation. Nevertheless, the question of whether coarsening behavior continues indefinitely at depth, or at which rate it slows down is still a question of interest, which would warrant further investigation. Along the 
same lines, the limited duration of the simulations also limited by ability to investigate coarsening for longer times. Especially for simulations with high $\mathrm{Ra}$, which required high resolutions, only simulation lengths of about a relative age of approximately $10 \tau_{S}$ were achievable.

Last but not least, to find a link of the planforms assumed by the underground dynamics to the surface pattern, a full 3D simulation of the system including interactions with a crust forming at the top would be necessary. Since computational cost increases tremendously for full 3D systems, this would not by achievable with our inefficient implementation of the simulation. Nevertheless, future work using a more efficient implementation has been started (see Sec. 6.2.1).

\subsection{Experiments}

The development and implementation of the analogue implementation of the advection-diffusion dynamics in the experiment comprised a significant part of my work on this project. Methods and observables are described in Sec. 3.2. Experiments are an important part of the investigation of the phenomenon of hexagonal salt ridge patterns in salt deserts, since they can both be used to develop protocols for the field investigations as well as to quantify the dynamics under controlled circumstances. As with direct hydrological evidence from the field, I tried to reproduce the circumstances found in the field as closely as possible. For this reason I used Hele-Shaw cells filled with sand instead of Hele-Shaw cells with a narrow gap between the plates, which could have simulated a porous medium as well. This complicated observation of the dynamics but allowed for the direct inclusion of the porous medium in the experiments.

Initially I did not expect coarsening of the dynamics to happen to such a significant extent. Therefore most of the protocols and setups I developed were not designed to accommodate a strong dependence of the system on age. In the following I will detail how this impacts the results of my work. Moreover I will explain why a direct observation of growing salt polygons in the laboratory remains elusive.

\subsubsection{Cell dimensions}

Depending on the evaporation rate imposed at the top, natural lengths in the experiments ranged from about $1 \mathrm{~mm}$ to $5 \mathrm{~cm}$. I performed most of the experiments in cells with dimensions of $40 \times 20 \mathrm{~cm}$. Therefore the observable domain in the experiments ranged between $8 \times 4 L$ to $400 \times 200 L$. I also conducted some experiments in cells with dimensions of $60 \times 20$ centimeters and the measured wavelength of the plumes did not change compared to the experiments conducted in the smaller setups.

Coloring experiments were performed at a depth of about $8 \mathrm{~cm}$, corresponding to a depth of $2-80 \mathrm{~L}$. While performing the individual coloring experiments, I had to keep the depth at which color was injected constant, due to limitations in the 
setup (the coloring tube had to be embedded at a given depth before the experiment was started). Additionally, I choose to embed the coloring tube at the same height of $8 \mathrm{~cm}$ in each experimental setup. Since I changed evaporation rate between coloring experiments, the measurement depth in terms of natural length scale was not constant for all experiments. In almost all cases coloring depth was far away from the top boundary and measurements were not impacted by re-initiating smaller plumes.

During many experiments I observed that the boundaries of the cell tended to "pin" downwellings: in about $90 \%$ of experiments, both boundaries had a downwelling next to them. An explanation for this observation could be the increased viscosity of fluid with higher salinity - similar to the discussion of pattern planform selection in Sec. 5.5 below: fluid with a higher salinity will have a higher viscosity and density and therefore sink downwards. Since the dynamics of the fluid will also minimize internal drag due to its own viscosity, fluid with a higher viscosity will preferentially move downwards at locations where drag is minimal. This could be the case at the walls of the experiment, since pores might be larger due to the solid boundary and the drag from moving against the solid, stationary polycarbonate wall will be lower than moving against a column of upwelling fluid.

While this pinning behavior of the dynamics can probably not be avoided in any experimental setup with a limited spatial extent, its effect should be taken into account. The dynamics in the observed domain showed between 5 and 8 downwellings (and therefore between 4 and 7 upwellings). If I assume that the presence of the solid walls at the cell sides are able to stretch or squeeze the dynamics by up to a wavelength, I expect to over or underestimate the wavelength of the dynamics by $10-25 \%$. Standard deviations of measured wavelengths are in the range of $20 \%$, therefore the error introduced by boundary effects could explain parts of the significant spread in experimentally observed wavelengths in Fig. 4.20.

\subsubsection{Direct observation of crust growth}

As described in Sec. 3.2, I focused on quasi-2D experiments for quantitative investigations of the scaling, complemented by a few 3D experiments for a qualitative assessment of the flows. While the results show very consistent results and comprise a clear indication of the link of subsurface dynamics to surface patterns, a direct observation of the growth of polygonal salt ridge patterns in the laboratory (as was possible at one of the field sites, as described in Sec. 4.7.2) would add to the quantitative analysis of the advection-diffusion dynamics.

During my work I experimented with the idea of growing artificial salt crusts. My goal was to observe the initial crystallization of salt in patches which, in the best case, should already exhibit a characteristic length scale. Nevertheless, the direct observation of growing salt crust patterns in the laboratory remains challenging. This is to some extent due to the long duration of the experiments, which make direct observations of the precipitation processes difficult. 
To a much greater extent this lack of direct evidence of ridge growth is due to the peculiarities of the crystallizing salt itself: since in the laboratory I only had access to finite size setups, boundary effects came into play. The fluid contained in the setup tended to creep onto edges and boundaries of the experiment, forming a thin film which evaporated quickly and cause salt to effloresce. These initial seed crystals of precipitated salt acted as a wick for further salty water and salt crusts grew from the initial precipitation locations. This resulted in salt crusts growing from the boundaries inwards in the 3D setup. In the Hele-Shaw setups, the salt precipitated first only at the edges of the cell and then grew in arches over the cell top. I tried to counter this effect by a hydrophobic coating of the cell walls but was not successful.

Salt crystallization experiments in three dimensional setups of similar size were conducted by [183]. Nevertheless, since the aim of their experiments was the study of crust erosion and not the subsurface dynamics, crusts were created by spraying saline water in small quantities on the top of the experiments and no observation of the impact of the subsurface dynamics on the crust growth was possible. Crusts observed by [183] also do not show any ridge patterns. Other experiments investigating the precipitation of salt in connection with porous media have been performed by [184, 185, 186]. Unfortunately, these experiments all investigated salt efflorescence on the surface and within a drying porous medium. Therefore the water front was constantly receding within the porous medium and buoyancy driven convective dynamics of the fluid in the porous medium were not observed.

I drove the experiments with evaporation rates far larger than the ones observed in the field to achieve results within manageable amounts of time. This in turn lead to small natural length scales $L$, often in the range of millimeters. Since I used artificial sand with a bead diameter of $100-200 \mu \mathrm{m}$ for most experiments, at these small natural length scales the continuum assumption for the porous matrix will start to break down. Any additional roughness at the surface will start to affect the initial locations of salt precipitation. If experiments to directly observe growth of salt crust and hexagons in the laboratory are repeated in the future, this effect has to be taken into account by driving the experiments with a suitable evaporation rate.

\subsection{Field results}

While literature on simulations and experiments investigating subsurface flow dynamics in porous media is plentiful, direct hydrological evidence for these flows from the field is rare and a connection between subsurface dynamics and surface expression is absent. Closest to direct evidence are field electrical resistance tomography (ERT) measurements of waste spillage and saline plumes [88, 152, 187]. Especially the study by [152] is interesting, since they investigated plumes of saline water below islands in the Okawango Delta in Botswana. The setting of a saline plume propagating in a porous medium in the field is quite similar to the salt lake 
problem. Nevertheless, the results presented by [152] investigate dynamics which occur on a timescale of several hundreds of years and on length scales of several hundreds of meters. This illustrates the severe drawbacks of limited spatial resolution. In addition, conditions in the Okawango Delta are most likely more forgiving and fragile ERT instruments less likely to fail than on the salt flats, where temperature gradients are large and winds are strong. Moreover, when using ERT, there is a lack of information about the underground characteristics such as grain size distributions and mineral species. With this work I close the gap in direct field evidence that allows for a thorough investigation of the characteristics of the porous medium, connects the subsurface dynamics with the surface expression and overcomes the limitations of ERT in the field.

The collection of evidence from the field is time-consuming and expensive. Nevertheless, I believe it is crucial to investigate these phenomena in nature, as there might be numerous influences on the dynamics in nature which cannot be modeled correctly in simulations or experiments. Results from this work can be used to calibrate models and lead to a number of questions, as detailed in Sec. 6.2, which can be investigated using both experimental and numerical as well as field research approaches.

In the following I will explain the significance and reliability of the results I obtained from my two field campaigns and quantify the errors of the measurements. I will also provide a more detailed description of the minerals found at the field sites and the methods used to quantify them. Last but not least I will briefly discuss the biological content of the samples collected in the field.

\subsubsection{Instability and salinity profiles}

In Sec. 4.2.2 I estimated the Rayleigh number in the field based on samples from 27 sites originating from three different salt pans in central California. Rayleigh numbers for all but one site lie well within the unstable region predicted by theory. The one outlier with $\mathrm{Ra}=9 \pm 4<\mathrm{Ra}_{c}=14.35$ could be explained by two different approaches: (1) the uncertainty of measurements and estimated quantities and (2) different boundary conditions. Especially the evaporation rate in the field is a large source of uncertainty which can significantly affect the Rayleigh number. The prediction of $\mathrm{Ra}_{c}=14.35$ is based on a calculation for a uniform flux boundary condition at the top. This corresponds to a porous medium saturated with salty water. Nevertheless, the managed sites at Owens Lake are known to be flooded with brine, which then slowly evaporates until the brine pool has completely dried, at which point a crust will form. For this "brine-pool" condition, a boundary condition of constant pressure (as listed in Tab. 4.1) would be more fitting. Repeating the calculation with this boundary condition would yield a slightly lower critical value of $\mathrm{Ra}_{c}=6.95$, which would suffice to place the one outlier within the unstable region in which convection can occur as well. 
I was not able to dig trenches at all sites, due to very wet conditions at most sites. In retrospect I discovered that the sampling protocol using the Dutch gauge corer contaminated the samples to an extent that made it impossible to recover the horizontal salinity signal (as discussed in Sec. 4.4.2). Therefore I only had access to samples from a very limited amount of polygon cross sections collected from trenches. The three salinity profiles measured at these sites are shown in Sec. 4.4.2. The number of profiles is arguably small and does not warrant a meaningful comparison of for example background salinities or salinity and spatial extent of the downwelling plumes between them.

Nevertheless, these results were obtained from previously undisturbed areas in the field which showed the surface patterns and show a statistically significant difference in the distribution of salt concentration below ridges as compared to the middle of the polygon. Moreover, I ensured that the origin of the signal is indeed the variation in salinity and not an artifact of the sampling or analysis protocol. In the days before I arrived at Owens Lake in early 2018, the region was subject to an unusually strong rainfall event (see Sec. 3.3.4). Since I expect an influence of the crust on the evaporation rate, the presence of ridges could also have influenced the rate at which the precipitated water seeps into the underground.

To invalidate this concern, I investigated the water content of the samples. Figure 5.2 (a) shows the salinity profile from Fig. 4.11 for reference. Panel (b) shows the water content in for each sample for an exemplary site (T27-S). Water content was calculated as $m_{\text {water }} /\left(m_{\text {sand }}+m_{\text {water }}\right)$, where the $m_{i}$ are the masses of water and sand in the sample respectively. There is no horizontal signal in the water content. Therefore there is no preferential input of water into the soil across the surface. A vertical signal in the water content is noticeable, as different layers of soil were comprised of grains with different grain sizes and therefore different porosities and contained varying amounts of water. Especially the layers at $70 \mathrm{~cm}$ and $80 \mathrm{~cm}$ depth show a greatly increased water content, which can be attributed to their proximity to the water table at a depth of approximately $80 \mathrm{~cm}$ and high clay content. For details of the stratigraphy of site T27-S, see Tab. A.19.

To exclude the possibility that the detected salinity signal is a product of human error and a varying implementation of the sampling protocol below the ridges, I also show the amount of sand per sample in panel (c) as proxy for the sample volume. Except for the bottom two lines, which reproduce the high water proportion signal there is no signal in the distribution of sample mass. The mass of the collected samples seems to follow a normal distribution.

\subsubsection{Redundant samples and measurements}

An additional measure to ensure the reliability of samples collected in the field and results obtained from their analysis is the collection of redundant samples and measurements. In some cases, I deliberately took samples from locations in close spatial proximity, analyzed them independently and later compared results. 


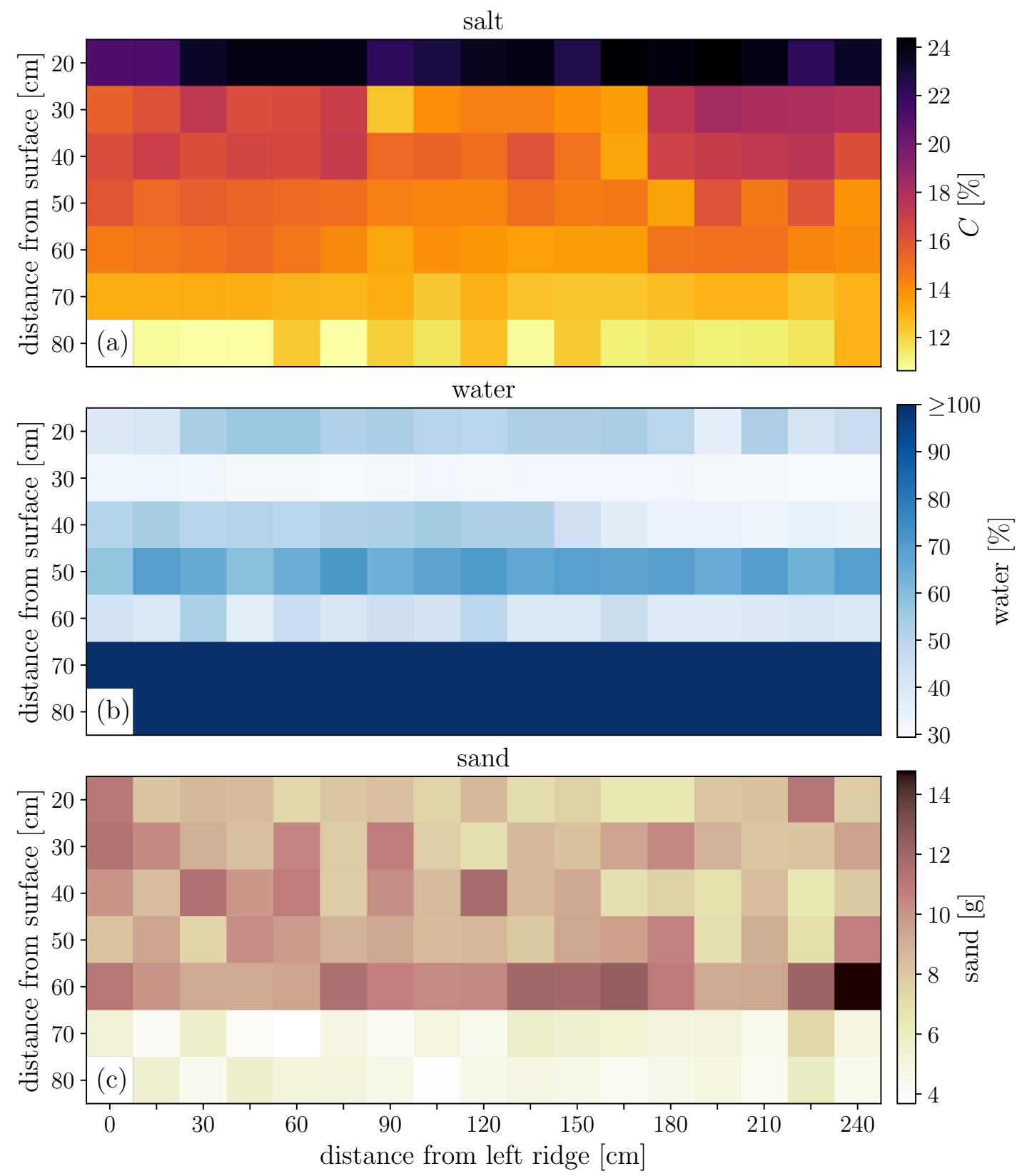

FIGURE 5.2: Content of samples taken at site T27-S. Panel (a) shows the salinity. Panel (b) shows pore water content in wt.\%. Panel (c) shows sand content per sample in grams.

Tab. 5.2 shows measurements of the Sauter diameter $d_{S}$ and salinity for samples collected from two cores drawn at a distance of approximately $5 \mathrm{~cm}$ from each other. Samples originate from site T16 and were collected during my second field campaign. Sauter diameters are very similar between the two samples. Since I used the Dutch gauge corer to collect samples, contamination of samples with brine and crust pieces is expected. This explains the rather large variation in measured salinity values between the two cores as well as the high salinity values in the top three samples, which exceed maximum saturation by up to a factor of two.

To investigate the reliability of the gravimetric measurement protocol for salinity, 


\begin{tabular}{c|cc|cc} 
depth $[\mathrm{cm}]$ & $d_{S}[\mu \mathrm{m}]$ & $d_{S}[\mu \mathrm{m}](\mathrm{V})$ & $C[\%]$ & $C[\%](\mathrm{V})$ \\
\hline 2 & 4.7 & 4.7 & 47 & 31 \\
10 & 4.7 & 6.0 & 32 & 61 \\
20 & 4.2 & 5.0 & 27 & 46 \\
30 & 12.0 & 13.3 & 21 & 19 \\
\hline
\end{tabular}

TABLE 5.2: Redundant samples collected at site T16 by coring. (V) indicates measurements from samples taken for validation purposes.

I used both redundant measurements of salinity as well as one site where I split the samples into two equal parts before processing. The two panels of Fig. 5.3 show the the salinity profiles of the two batches of samples. Samples were collected from site T36-3 (3) during my first field campaign. Samples were also collected with a Dutch gauge corer, but since the samples were only split after collection, contamination by brine from the surface should be the same between both parts of every sample. Salinity profiles are very similar between the two batches with the main variations in the first two rows of samples. This could be due to contamination in the form of larger salt crystals, which were not split between the samples but ended up in one or the other part of the sample and were later dissolved during further processing and measurement.

The amplitude of the salinity signal in the redundantly analyzed samples is $A_{S}=A_{\max }-A_{\min } \approx 26 \mathrm{wt} . \%$. Including the first two rows of samples, the maximum deviation between the two batches is about $5.7 \mathrm{wt} . \%$ or $0.22 A_{S}$. Excluding the first two rows, the maximum deviation is about $2.5 \mathrm{wt} . \%$ or $0.09 A_{S}$ and the mean deviation is about $1 \mathrm{wt} . \%$ or $0.04 A_{S}$. From experiments I know that the expected salinity signal is in the range of about $8 \mathrm{wt} . \%$ - although given a salinity gradient between background and maximum salinity which is about twice as large as the gradient measured in the field. Therefore I can conclude that my processing accuracy is good enough to detect any signal present in the samples collected in the field with reasonably high confidence.

As described in Sec. 3.2.4, for each gravimetric measurement of salt content I measured the weight of the salt both directly (as precipitated minerals in a separate crystallization dish) as well as indirectly (as weight missing from the remaining solid sample components). These two measurements were very consistent most of the time, with the indirect measurement being a little more reliable due to the tendency of the drying salt to trap water.

For samples collected during the first field campaign I also measured the salinity by measuring conductivity of the minerals in solution in a known amount of water. These measurements reproduced the same trends as the gravimetric measurements but were significantly less reliable. This was probably due to the fact that conductivity measurements not only depend on the total amount of dissolved salts but also the type of the ions, which is rather variable.

While the number of salinity profiles with a high enough accuracy to detect horizontal variations in salinity is small, the individual gravimetric measurements are 


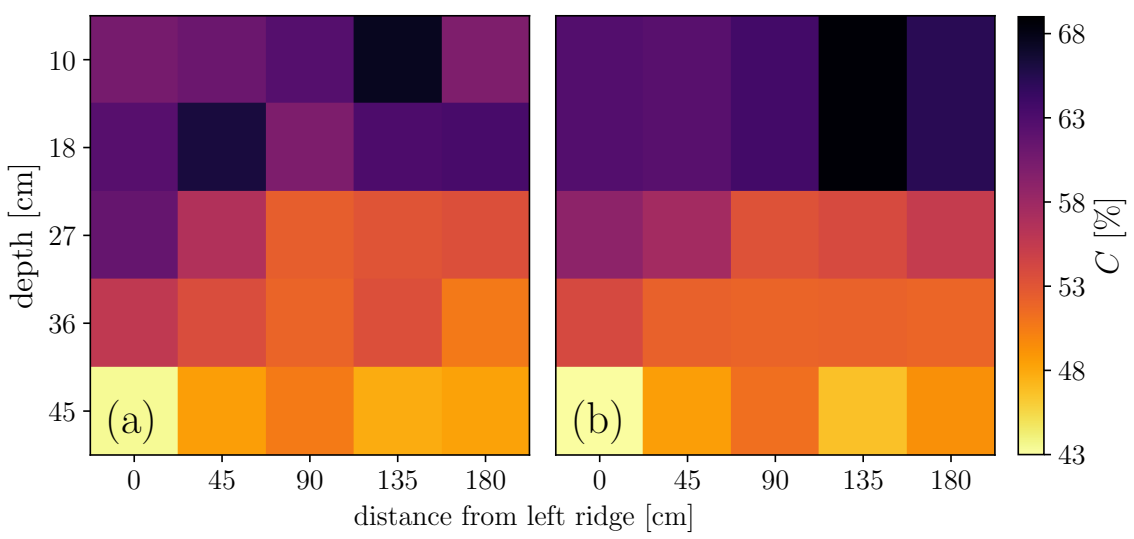

FIGURE 5.3: Samples from site T36-3 (3) collected during the first field campaign in 2016 were split into two equal parts before being processed in the laboratory. Panels (a) and (b) show the salinity values measured for each of the two sample groups and allow for a comparison of the accuracy of salinity measurements. Color code indicates salinity in wt.\% of pore water.

very reliable, statistically significant and consistent with the experimental and numerical results as well as the theoretical predictions. I conclude that it is warranted to use them as strong evidence for the existence of density driven convection below polygonal salt ridge patterns visible at the surface.

\subsubsection{Salt crusts}

Finding the link between subsurface dynamics and crust formation and growth most certainly also includes specifics about the crystallization of salt. In terms of the crystallization and volume of the precipitated crust, different salts show vastly different behaviors. Most prominently among them, Mirabilite [188] (sodium sulfate decahydrate) is a hydrated crystal that binds 10 molecules of $\mathrm{H}_{2} \mathrm{O}$ per molecule of $\mathrm{Na}_{2} \mathrm{SO}_{4}$ at low temperatures. At approximately $13^{\circ} \mathrm{C}$, a binary system of $10 \mathrm{wt} . \%$ Mirabilite + ice will transition to anhydrous sodium sulfate + liquid [189, 190]. Estimating the exact proportion of Mirabilite in the crust is difficult but a proportion between 8 wt. $\%$ and $15 \mathrm{wt} . \%$ seems warranted, given my analysis of the minerals present in the crust (see Appendix B). I encountered the phase change of Mirabilite while present at the field sites as temperatures rose from below $0^{\circ} \mathrm{C}$ in the morning to about $20^{\circ} \mathrm{C}$ around mid-day. Effects like this have to be taken into account when modeling crust growth: for example the volumetric growth of ridges is not necessarily directly relatable to a salt flux to the surface, since ridges could be significantly more porous, due to repeated swelling and shrinking during diurnal temperature cycles. Therefore, an accurate description of crust mineral composition as well as experiments investigating crust growth with mixtures of different salts are of interest.

While it is beyond the scope of this work to model crust mechanics and chemistry and its connection to the subsurface dynamics, I did a thorough quantification of the minerals present in the crust and pore water. Results and interpretations based 
on quantitative XRD and ICP-OES analysis (as described in Sec. 3.3.3) are given in Appendix B.

\subsubsection{Biological sample content}

While measuring salinity content of the samples collected in the field, I noticed signs of organic components. This makes sense in the light of the visible traces of microorganisms such as Dunaliella Salina at the sites (see Sec. 3.3.3 for more details). Images of microorganisms cultivated from the samples taken at Owens Lake are also shown in Appendix C.

I assessed the amount of organic matter in a selection of samples which were among the samples appearing to have the highest proportion of organic components (see Sec. 3.3.3 for protocol details). Results for samples from site T32-1-L1 (1) are shown in Fig. 5.4. Organic components are given as ratio of organic component mass to salt mass or sand mass of the sample respectively.

I note that organic components seem to decrease with depth. This seems consistent, since most microorganisms including Dunaliella Salina require light to photosynthesize and thrive, which will only be accessible close to the crust. Moreover, the amount of organic matter seems to be significantly higher in the salt proportion of the sample. This means that most of the organic materials were dissolved dur-

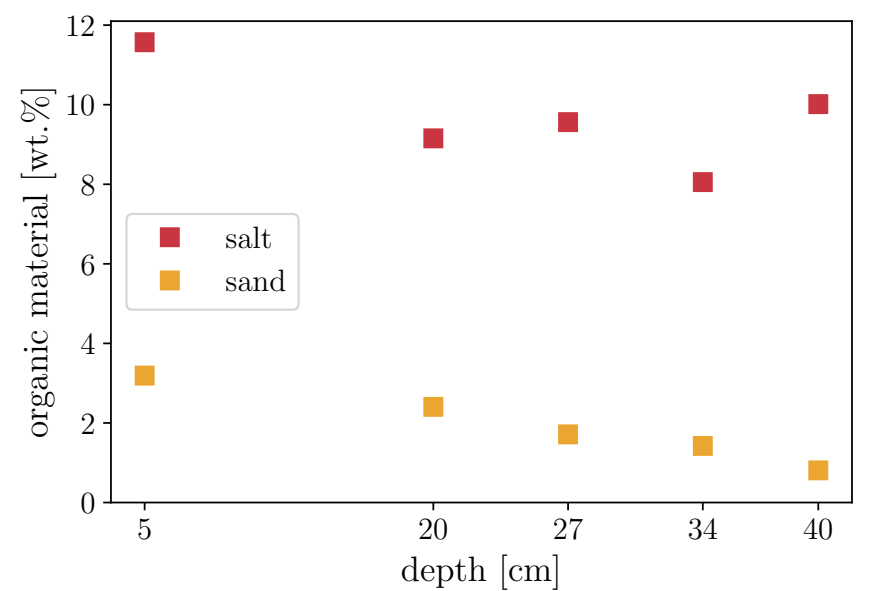

FIGURE 5.4: Organic matter proportions for the salt (red) and sand (orange) part of samples from site T32-1-L1 (1) over depth.

ing the dilution phase of the salinity measurement protocol and subsequently separated from the solid (sand) part. This also means that organic components probably contribute naturally to the density of the fluid and I do not have to treat them separately when modeling the fluid flow. Modeling phenomena such as bioconvection or chemo/phototaxis of microorganisms is outside the scope of this work. Given the overall small amount of organic components in the samples measured, it seems warranted to neglect their influence on the overall dynamics.

\subsection{Why hexagons?}

So far I have tried to show why instability has to occur in a given field setting and what wavelength the instability will select, given feedback from the crust. During my investigation, I have always reduced the system to quasi-2D to facilitate analytical, numerical and experimental treatment. Nevertheless, a big part of the iconic 
appearance of these patterns in nature is their regular and hexagonal shape. This aspect is lost in a $2 \mathrm{D}$ representation of the system.

The question of the planform selection of a convective processes has been addressed in the literature before. Especially for a system which is a very close relative to the salt playa system, the Rayleigh-Bénard convection in a porous medium, a selection of analytical and experimental investigations of planforms exist [191, 192, 193]. Rayleigh-Bénard convection is driven by a temperature gradient between a hot plate at the bottom and a cold plate at the surface. As temperature increases, density and viscosity of the fluid decrease. In my system, the equivalent to temperature is salinity, which is high at the top and low at the bottom. As salinity increases, density and viscosity increase as well. Therefore both temperature and salinity gradient driven systems are expected to show qualitatively similar behavior. A numerical investigation in a porous medium, which allows for an inspection of the selected planforms, was conducted by [194] and [102]. The latter also reported significant coarsening throughout the simulation.

As fluid starts to rise/fall because of density differences, strain in the fluid builds because of viscous drag between the rising/falling flows. This strain is minimized if fluid flows minimize the circumference-to-area ratio of their envelope [195]. Here the envelope can be understood as regions in-between upwards and downwards fluid motion, where $U_{Z}=0$. The circumference-to-area ratio would be minimal for a circular envelope of the flows. Circles are not space-filling, therefore hexagons are selected, since they are the space-filling geometric form with the best circumference to area ratio.

The question of flow orientation remains: will the downwelling be located in the center or at the edges of a hexagonal cell? This question can be answered by the direction of the dependence of fluid kinematic viscosity on density. For both the temperature as well as the salinity driven system, viscosity increases with density. Since the center of the hexagon is the region of highest strain in the flow system, fluid with the lowest viscosity will select the center, whereas fluid with the highest viscosity will select the edges of the hexagon [195]. For the salt playa system this means that theory predicts upwellings in the hexagon centers and downwellings at the edges. This is consistent with my findings in the field, presented in Sec. 4.4.2.

\subsection{Summary}

In this chapter I revisited the assumptions made during modeling of the system, specifically the assumption of constant viscosity and the unimportance of dispersion for the salt playa problem. I related the model to the parameters measured in the field and illustrated, why the assumptions are warranted and the associated effects negligible for my model. 
I discussed the limitations of the simulations due to their two-dimensional nature, which does not enable me to investigate the planforms of the simulated dynamics. I related the results of my simulations to behavior found in similar systems, which has been described in the literature. Most importantly, the different regimes of initial instability, plume growth, coarsening and re-initiation of plumes at the surface have been reported before, especially in $\mathrm{CO}_{2}$ sequestration literature, and my results are consistent with these findings.

For the analogue experiments, I discussed the limitations of the setup due to the limited size of the setups. Limitations include possible effects on the measured wavelengths of the convective dynamics due to quantization of the dynamics. Moreover, the direct observation of salt crusts forming ridges in the experiment was challenging, due to boundary effects in the experiments. A comparison to the existing literature on salt precipitation on the surface of a porous medium shows, that to this date, no observation of polygonal salt ridge patterns in the laboratory was possible. In general, existing literature on experiments either only investigates subsurface dynamics or the salt precipitation on the crust. A connection of the both phenomena is lacking.

I discussed my results from the field study under the perspective of the limited number of data points I collected. I described the measures I implemented to assure reliability and accuracy of the collected data and exclude any effect by human or systematic bias or error. I also discuss the importance of different minerals present in the field for the formation and behavior of salt crusts. Specifically, I discuss the particularities of Mirabilite which swells and shrinks with diurnal temperature changes. I also included a short discussion on the significance of biological components in the samples collected in the field, which leads me to the conclusion that their impact is negligible.

Finally, I revisited the question of why the patterns observed in nature are hexagons. I drew from literature on the investigation of planforms in Rayleigh-Bénard convection to illustrate, why hexagonal forms are preferred to minimize the internal strain in the fluid. For this process, the density dependence of the fluid's viscosity is important, since it will directly influence the strain. Following these considerations I predicted that downwellings will occur along the ridges where the viscosity and therefore density is highest, whereas upwellings will occur in the polygon centers, where viscosity is low. This is consistent with my findings of plumes of high salinity in the field, which are co-located with the salt ridges visible at the surface. 


\section{Chapter 6}

\section{Conclusion}

With this chapter I conclude my investigation of the mechanism driving the emergence of hexagonal salt ridge patterns in in salt deserts. I summarize my hypothesis formulated in section 1.3.1 and the evidence I presented in chapter 4. In the second part of this chapter I provide an outlook of possible research questions that my work has inspired.

\subsection{Summary}

In the introduction to this work I present my hypothesis for the driving mechanism underlying the pattern formation process in salt deserts: convective dynamics in the ground below the ridge patterns. I model the system as an advection-diffusion process in a porous medium, which interacts with the crust that precipitates at the surface. The often hexagonal ridge patterns at the surface are located at the edges of the convection cells, where water of high salinity flows downwards. With this model I extend the growing list of geomorphological phenomena such as fairy circles, columnar joints or ice wedge patterns, which were successfully explained as a result of an instability in a dynamical systems.

\subsubsection{Instability}

The first prerequisite for a successful explanation of the pattern formation process as outcome of an instability in a dynamical process is to verify, that the conditions for instability are fulfilled in nature.

I first adapt existing models for convection in porous media to fit the boundary conditions found in the field. The system is described by the governing equations of the mass and momentum balance for fluid and salt transport. Nondimensionalization allows me to scale the resulting system by a single, dimensionless number - the Rayleigh number - which describes the force balance between buoyancy and dissipation in the system. The Rayleigh number is comprised of ratio of the permeability, the density gradient and gravity to the evaporation rate, the viscosity and the porosity of the system. I employ a linear stability analysis of the governing equations to find the critical Rayleigh number above which a perturbation in the system does not decay anymore, and at which the system becomes unstable and starts to exhibit 
buoyancy driven convection. Alongside the critical Rayleigh number I also find the wavenumber of the most unstable perturbative mode.

To assess whether the situation in the field shows a higher than critical Rayleigh number, I conducted two field campaigns to salt deserts exhibiting polygonal patterns at the West coast of the USA. During these campaigns at Owens Lake, Badwater Basin and Bristol Playa, I collected samples to measure the subsurface salt concentration, the density gradient, the diffusivity, the permeability and the porosity of the soil below the salt ridge patterns. Using literature values for the evaporation rates, I calculate the Rayleigh numbers for 27 individual field sites. All but one site show a Rayleigh number above the critical value necessary for the onset of instability. Often, the sites were several orders of magnitude above the expected onset conditions.

\subsubsection{Density stratification}

To compare systems at different Rayleigh numbers, I introduce a timescale $\tau_{S}$, defined as the time it takes until the salinity at depth $Z=-1$ reaches a value of $S \geq 0.5$, which is characteristic for a system at a given Rayleigh number. The relative system age $\tau_{S}$ scales as a power law with $\mathrm{Ra}-\mathrm{Ra}_{c}$ and an exponent of approximately -1.3 . Systems with different Ra but the same relative age show the same state of coarsening.

The steady state solution of the salinity distribution allows me to predict a length scale $L^{\prime}$ of the salinity decay with depth at the onset of convection. I measure this length scale in simulations which show convective dynamics and find that $L^{\prime}$ depends both on time as well as on the system Rayleigh number.

I find salinity gradients which correspond to an exponential decay with a length scale in the range of $L^{\prime}=0.7-2.4$ both in an analogue experiment conducted in a Hele-Shaw cell, as well as at nine different field sites. The existence of a vertical density gradient allows the conclusion that density gradient driven convective dynamics should occur both in the experimental setting as well as in the field.

\subsubsection{Convection}

To directly show the existence of convective dynamics in the field, I collected samples along horizontal cross-sections below polygons to compile salinity profiles. I find statistically significant evidence for plumes of high salinity at all three field sites which I sampled with a high enough resolution to detect horizontal salinity gradients. The high salinity plumes are located directly below the ridges visible at the surface. This is consistent with the prediction of downwellings at regions of high salinity, since salinity increases the viscosity of the fluid.

Additionally, I observe convective dynamics in a number of analogue experiments conducted in Hele-Shaw cells and find that all experiments with $\mathrm{Ra}>\mathrm{Ra}_{c}$ show convective dynamics. I confirm that the convective dynamics are indeed driven 
by salinity gradients by visualizing the flow dynamics and measuring the salinity at up- and downwellings of the flow.

\subsubsection{Scaling}

I find that while the theory correctly predicts a scaling of the wavenumber with the Rayleigh number directly after the onset of instability, this dependence diminishes as the system ages and coarsens. As previously found in simulations and experiments described in the literature of related systems, the dynamics are subject to pronounced coarsening with time.

I trace the coarsening behaviour in numerical simulations for relative system ages between 1-99 $\tau_{S}$ and in experiments up to $5 \cdot 3^{4} \tau_{S}$. Coarsening slows down as the system ages and approaches the critical wavenumber predicted by the linear stability analysis in very old systems. Wavenumbers for systems with advanced coarsening cluster in a range of $a=0.2-2.5$.

The measurement of the pattern wavelengths using terrestrial laser scanning in the field allows me to directly compare the selected wavelength in nature with the wavelengths predicted by theory, and found in numerical simulations of the system and experiments. Wavelengths measured in the field lie in between the wavelengths measured for younger and older system in the simulations and also cluster in the wavenumber range of $a=0.5-2$. I find a clear correlation between ridge height and pattern wavelength, which I correlate with polygon age. The expressed coarsened wavelength places the estimated age of the patterns found in nature at $\tau \geq 10 \tau_{S}$. For the patterns in nature, flooding events, which happen roughly every 1-3 years and diminish ridge heights or wipe out patterns completely, have been recorded. An age of one year for a system with the median Rayleigh number found in the field corresponds to an age of $11 \tau_{S}$, which is consistent with the possible ages predicted based on the measured wavelengths.

The coarsening behavior and selection of wavenumbers in a very narrow range can explain, why these salt polygons have roughly the same size all over the world, independent of local circumstances. The necessary critical Rayleigh number for the onset of convection is achieved rather easily by soils which are not too fine-grained - a condition which is fulfilled in many areas with fluvial deposits. After onset, the dynamics are very robust to changes in the Rayleigh number, always coarsen with time and approach the same critical wavenumber $a_{c}$. The higher the Rayleigh number, the faster the coarsening process will place the selected wavelength of the patterns in the range of preferred wavenumbers.

\subsubsection{Feedback}

Next to the coarsening, the numerical simulations also reveal a regime of plume re-initiation after the initial onset of instability and first generation of downwelling plumes. In this regime, small plumes originate at the surface and move horizontally 
until they are drawn into one of the roots of the larger downwelling plumes. The roots of the large plumes also fluctuate slowly in space as time advances. Both phenomena would hinder the emergence of well-defined patterns at the surface, since the dynamics in the underground would need to stay stationary for a sufficiently long period of time to support crust growth at the surface. To overcome this apparent contradiction, I introduce a potential feedback mechanism between the crust at the surface and the underlying dynamics.

The feedback mechanism acts on the evaporation rate and modulates the initially uniform flow at the surface. I find evidence in the field, pointing to a temperature and humidity modulation below ridges, which in turn could influence evaporation rate. I connect these findings to evidence in literature [174] which indicates that evaporation from a porous medium can be influenced significantly by changes in ambient temperature and humidity.

I investigate the feedback mechanism in numerical simulations and find that even an evaporation rate modulation with a very low amplitude is able to pin the positions of the downwelling plumes for a considerable amount of time, if the wavelength of the modulation is selected correctly. For low amplitudes I found pinning only for wavenumbers in the window of $a=0.5-1.5$, which is the same wavenumber range selected by the patterns in the field. Moreover, the pinning is also able to suppress the re-initiation regime for a prolonged period of time. Pinning lasts for a period of time which corresponds to approximately 1300 days in nature - long enough to support the growth of pronounced salt ridges and polygonal patterns, which reach considerable height over the course of 3-6 months, as indicated by timelapse photography I acquired at one of my field sites.

\subsubsection{Concluding remarks}

With my work I am able to explain the driving mechanism of salt ridge patterns found in salt deserts, which I find to be buoyancy driven convection in the soil beneath the patterns. I find the necessary conditions for onset and scaling of the convection and explain the timescales on which the patterns grow. Such patterns can be found in Chile, Iran, the Dead Sea and at the West coast of the US, with my model I am able to explain, why these patterns always have similar dimensions.

\subsection{Outlook}

\subsubsection{Simulations}

Extension to 3D: As mentioned in Sec. 5.5 and Sec. 5.2.2, a two-dimensional representation of the dynamical system is not able to express the three-dimensional patterns which emerge in nature. The full extent of the dynamical process including 
planform selection is only accessible in a three-dimensional representation. An investigation of the selected planforms would allow for a comparison of pattern statistics such as coordination numbers and numbers of edges of polygons with the surface relief scans from the field. A recent collaboration with Cédric Beaume, who has access to more sophisticated numerical tools, has allowed for a preliminary investigation of the full three-dimensional dynamics of the system. In Fig. 6.1 (a) I show a horizontal cross-section through such a simulation at $Z=-1$ shortly after the onset of convection. In panel (b) I show the coarsened early stage pattern assumed by the downwelling, strongly resembling the patterns observed in nature. Panels (c) and (d) show late stages of the simulations where the convection cells coarsened further and assumed quadratic forms. This could be due to the small extent of the system in combination to the periodic boundary conditions, which could cause the boundaries of the convection cells to align with the symmetry of the domain. The quadratic form could also be due to the fact that the density dependence of the viscosity in the simulated system was neglected and therefore geometric forms with sharper edges than hexagons, such as triangles and squares, are not penalized by the internal strain of the fluid.

Once a quantification of the planforms in the simulation is possible, surface reliefs measured in the field could be quantified as well and compared to the results from the simulation. This could be easily achieved by vectorizing the surface relief scans and representing the ridges as networks and graphs, as has been demonstrated for example by [196] for different networks. If a robust measure of the similarities between planforms selected by a certain dynamical process and the patterns expressed in the surface is found, this could be used to draw conclusions about subsurface processes in regions were only surface information is accessible.

Coupled simulation of crust and convective dynamics: Additionally, a simulation of the dynamics of the growing crust, including surface precipitation as well as dissolution of salt and an exchange with the underlying convecting fluid could further contribute to the understanding of the feedback mechanism between subsurface and surface. Such a dynamic boundary condition could be used to inform additional experiments in the field but also to bring our understanding of the system to a point where more detailed analogue laboratory experiments which directly demonstrate pattern growth are possible.

Hysteresis and targeted convection: In the literature, there is evidence for hysteresis in an advection-diffusion system similar to the one described in this work [118]. Specifically, they found that lowering and increasing the Rayleigh number during a simulation yields different wavenumbers of the convective dynamics at the same Rayleigh number. In the timelapse images of growing polygons I recorded in the field, I noticed a certain preferential selection of locations for ridge growth after an 

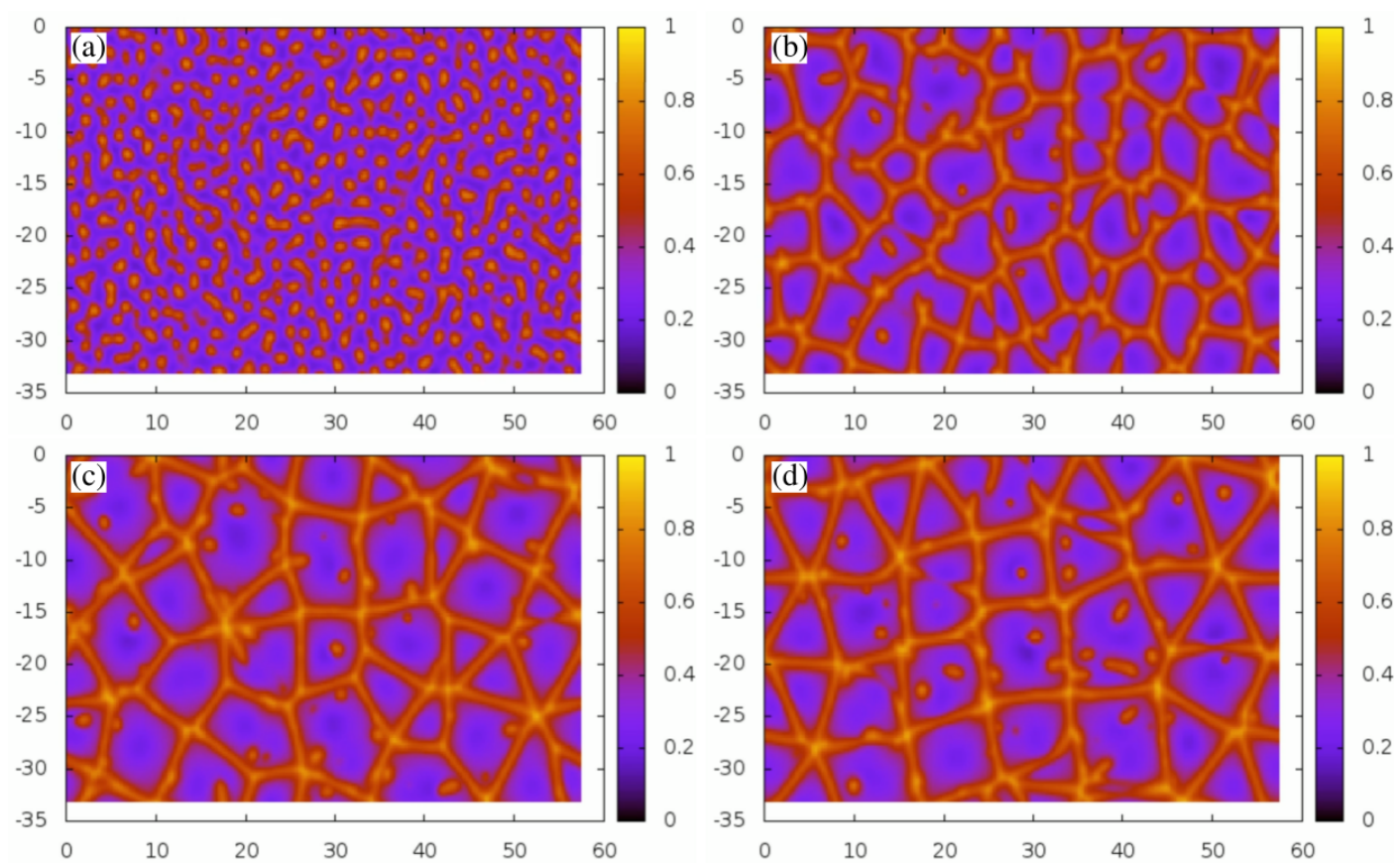

FIGURE 6.1: Snapshots of planforms at $Z=-1$ in three-dimensional simulations of the buoyancy driven porous media convection at $\mathrm{Ra}=100$, conducted by Cédric Beaume. The colormap indicates salinity $S \in[0,1]$. (a) Early stage of the simulation shortly after the onset of convection. (b) Simulation after some coarsening showing convection cells very similar to the ridge patterns observed in nature. (c) and (d) late stages of the simulation, showing a transition to square convection cells. Images courtesy to Cédric Beaume, University of Leeds.

inundation event where ridges had previously existed. This could be both an indicator for hysteresis as well as for an interaction between the surface and subsurface dynamics. Hysteresis could be easily studied by changing simulation parameters throughout a simulation run and quantifying the ensuing dynamics.

Top boundary condition: I found that a modulation of the top boundary condition with a low amplitude is enough to significantly influence the spatial properties of the subsurface dynamics. Apart from a spatial modulation, a temporal modulation could also be investigated to model diurnal and seasonal temperature changes and investigate the selection of pattern wavelengths under conditions of increased noise. It would be interesting to clarify whether the effect also persists for an advection-diffusion system without the porous medium and for immiscible flows. If this is the case, the fact that rather small and local modulations are capable of producing long-range effects should be considered in the modeling of other systems of similar dynamics, such as the convective dynamics of the atmosphere and sea.

Targeted convection: An effect similar to the modulated top boundary could probably also be achieved by a non-uniform permeability distribution throughout the porous medium. The targeted control over the position of the downwellings in the dynamics could be used to develop applications which are able to reliably 
control and shape convective dynamics with relatively little effort. The ability to target specific locations for the downwellings could be of use for applications in $\mathrm{CO}_{2}$ sequestration, where certain geological formations are of more interest than others. Along the same lines, targeted up- and down-flow of air could also be of interest for the design of room ventilation systems and findings could be used to inform architectural decisions.

\subsubsection{Experiment}

Control over system age: To improve on the quantification of the dynamics in the experiment, a new round of experiments with better control over the system age could be conducted. In addition, circular cells would allow for the mitigation of boundary effects in the system. I already started to implement circular experiments, one example is visualized in Fig. 6.2 (a), but did not finish collecting results at the point where this work was written.

Flow visualization: Visualization of flows in a porous medium by injection of a thin line of color into the experiment is, to my knowledge, a novel approach. Several extensions of the visualization protocol are imaginable. For example, coarsening could be quantified by embedding coloring tubes at different heights in the same experimental setup and quantifying the wavelength dependence on system depth.

Conductance based salinity measurements: Since in the experiments complete saturation of the porous medium with fluid is always guaranteed and the type of diffusing ion can be controlled, conductance based measurements of the salinity are feasible. By attaching a fine grid of conductance sensors onto one of the walls of the HeleShaw cells, a better spatial and temporal resolution of the salinity distribution could be measured. This would also reduce the potential interference with the convective dynamics caused by the

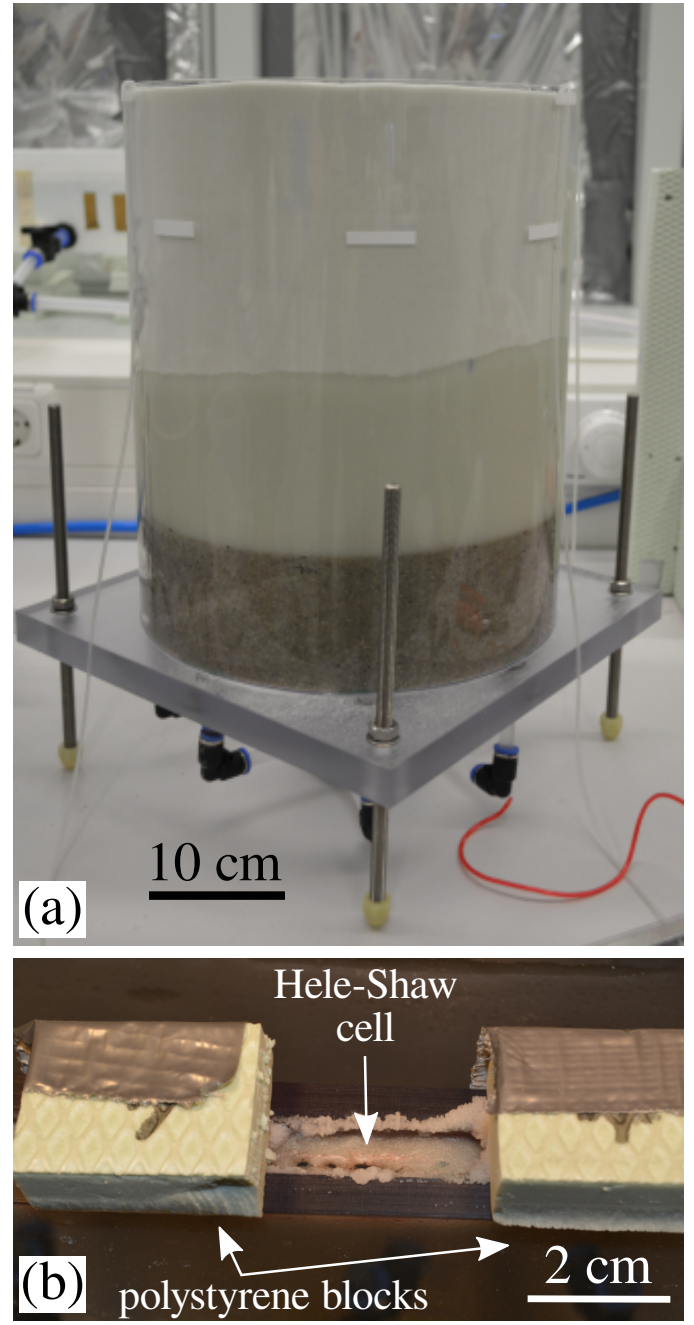

FIGURE 6.2: Ideas for improved and novel experimental setups. (a) A circular Hele-Shaw cell and (b) modulation of the evaporation rate with polystyrene blocks. 
presence of the coloring tube and dependence on the diffusivity of the tracer color. Moreover, higher system ages would be accessible to sustained monitoring of the dynamics, because saturation of the setup with color could be avoided.

Feedback in the experiment: To implement the feedback idea developed in the numerical simulations as modulation of the top boundary condition, evaporation could be locally reduced or altered. A proof-of-principle test of this idea was attempted by blocking evaporation locally with polystyrene plugs (see Fig. 6.2 (b)) but I was not able to successfully match the plug distance to the preferred system wavelength.

Different salts: Since different minerals such as sulfates play a large role in salt deserts in nature, incorporating them into the experimental setups seems warranted as well. A very simple extension of the experiments would be a comparison of the existing data on the selected wavelengths with experiments where sodium chloride is replaced with sodium sulfate, which has a molecular diffusion coefficient which is about three times smaller than that of sodium chloride.

Large-scale 3D experiments: Apart from experiments in a Hele-Shaw cell, experiments which try to directly reproduce polygonal ridge pattern growth in the laboratory are imaginable. For this purpose, experiments would need to be large enough to mitigate the influence of boundaries on the precipitation process. This would require significant effort in building the experiment and a significant amount of materials and energy. Nevertheless, equipped with sensors, such a setup could be a worthwhile addition to field experiments.

\subsubsection{Field}

Investigation of crust mechanics: The next step for an investigation of the dynamics in the field would be the clarification of the exact interplay between crust and subsurface dynamics. Parameters such as temperature and humidity should be measured below ridges and compared to measurements in the polygon centers. Additionally, timelapse images of crust growth for an extended period of time could clarify, how fast and during which season ridges grow and if the pattern coarsens further once the first ridges have grown. Additionally, the minerals forming the crust might also have a significant influence on its morphology. Especially sodium sulfate is of interest here, since it switches between Thenardite and Mirabilite as temperature decreases, significantly increasing its volume. Given the large diurnal temperature variations in deserts, this could play a significant role in crust dynamics.

Coloring experiments in the field: Timelapse photography would also allow for a direct observation of flows in the field, if done for a long enough period of time. For a known diffusion coefficient and evaporation rate, the natural length scale is 
$L=D / E \approx 0.3 \mathrm{~cm}$. The time it takes the fluid to travel one natural length scale is $T=D / E^{2} \approx 600$ days. One could imagine a coloring experiment, where color is introduced at depth and observed as it re-surfaces with the flow. This could give an indication of the flow patterns in the underground. An observation of any significant flow movement would take a long-term observation effort of the crust over the course of a year.

Conductance based real time flow investigation: Real-time imaging of underground flows, especially at very wet sites, could also be achieved with electrical resistance tomography. Recent advances in the technology make measurements with the required spatial resolutions possible [153]. For measurements over extended periods of time under field conditions, robustness of the methodology is of great importance. Nevertheless, this engineering challenge could probably be overcome.

Other field sites: Last but not least, an investigation of different field sites would be of interest. While I have data from three field sites in the US, these patterns are know to emerge in salt deserts on different continents and in very different climates. A comparison between the patterns and conditions from the different salt deserts of the world could give an indication of possible mechanisms influencing pattern formation which are not yet known to us or demonstrate the universality of the pattern wavelengths. To this extent, a collaboration with Jo Nield from the University of Southampton, who lead multiple research campaigns to salt flats in Namibia, which exhibit similar patterns, could be pursued. 



\section{Appendix A}

\section{Field site descriptions}

\section{A.1 Field site locations}

\begin{tabular}{|c|c|c|c|c|c|}
\hline Location & Site & Latitude & Longitude & Year & \# Samples \\
\hline Death Valley & Badwater (1) & $36^{\circ} 13.651^{\prime}$ & $-116^{\circ} 46.723^{\prime}$ & 2016 & 25 \\
\hline Death Valley & Badwater (2) & $36^{\circ} 13.674^{\prime}$ & $-116^{\circ} 46.735^{\prime}$ & 2016 & 25 \\
\hline Death Valley & Badwater (3) & $36^{\circ} 13.665^{\prime}$ & $-116^{\circ} 46.820^{\prime}$ & 2016 & 25 \\
\hline Death Valley & Badwater (4) & $36^{\circ} 13.660^{\prime}$ & $-116^{\circ} 46.903^{\prime}$ & 2016 & 25 \\
\hline Death Valley & Badwater (5) & $36^{\circ} 13.654^{\prime}$ & $-116^{\circ} 47.036^{\prime}$ & 2016 & 25 \\
\hline Mojave & Bristol Playa & $34^{\circ} 27.762^{\prime}$ & $-115^{\circ} 44.573^{\prime}$ & 2016 & 25 \\
\hline Owens Lake & T10-3 & $36^{\circ} 23.147^{\prime}$ & $-117^{\circ} 56.772^{\prime}$ & 2018 & 118 \\
\hline Owens Lake & T16 & $36^{\circ} 23.953^{\prime}$ & $-117^{\circ} 56.454^{\prime}$ & 2018 & 144 \\
\hline Owens Lake & T2-4 & $36^{\circ} 20.803^{\prime}$ & $-117^{\circ} 58.642^{\prime}$ & 2016 & 25 \\
\hline Owens Lake & T2-5 (1) & $36^{\circ} 21.055^{\prime}$ & $-117^{\circ} 58.824^{\prime}$ & 2016 & 25 \\
\hline Owens Lake & T2-5 (2) & $36^{\circ} 20.895^{\prime}$ & $-117^{\circ} 58.740^{\prime}$ & 2016 & 35 \\
\hline Owens Lake & T2-5 (3) & $36^{\circ} 20.877^{\prime}$ & $-117^{\circ} 58.711$ & 2018 & 144 \\
\hline Owens Lake & T25-3 (1) & $36^{\circ} 27.039^{\prime}$ & $-117^{\circ} 54.510^{\prime}$ & 2018 & 112 \\
\hline Owens Lake & T25-3 (2) & $36^{\circ} 28.383^{\prime}$ & $-117^{\circ} 54.957^{\prime}$ & 2018 & 342 \\
\hline Owens Lake & T27-A (1) & $36^{\circ} 29.302^{\prime}$ & $-117^{\circ} 55.953^{\prime}$ & 2016 & 25 \\
\hline Owens Lake & T27-A (2) & $36^{\circ} 29.061^{\prime}$ & $-117^{\circ} 55.602^{\prime}$ & 2016 & 25 \\
\hline Owens Lake & T27-A (3) & $36^{\circ} 29.112^{\prime}$ & $-117^{\circ} 55.804^{\prime}$ & 2018 & 88 \\
\hline Owens Lake & T27-S & $36^{\circ} 28.549^{\prime}$ & $-117^{\circ} 54.994^{\prime}$ & 2018 & 153 \\
\hline Owens Lake & T29-3 (1) & $36^{\circ} 29.955^{\prime}$ & $-117^{\circ} 55.999^{\prime}$ & 2016 & 35 \\
\hline Owens Lake & T29-3 (2) & $36^{\circ} 29.960^{\prime}$ & $-117^{\circ} 55.962^{\prime}$ & 2016 & 25 \\
\hline Owens Lake & T32-1-L1 (1) & $36^{\circ} 53.897^{\prime}$ & $-117^{\circ} 57.209^{\prime}$ & 2016 & 35 \\
\hline Owens Lake & T32-1-L1 (2) & $36^{\circ} 32.354^{\prime}$ & $-117^{\circ} 57.218^{\prime}$ & 2018 & 128 \\
\hline Owens Lake & T32-1-L1 (3) & $36^{\circ} 32.337^{\prime}$ & $-117^{\circ} 57.204^{\prime}$ & 2018 & 126 \\
\hline Owens Lake & T36-3 (1) & $36^{\circ} 29.953^{\prime}$ & $-117^{\circ} 58.505^{\prime}$ & 2016 & 25 \\
\hline Owens Lake & T36-3 (2) & $36^{\circ} 30.050^{\prime}$ & $-117^{\circ} 58.518^{\prime}$ & 2016 & 25 \\
\hline Owens Lake & T36-3 (3) & $36^{\circ} 29.724^{\prime}$ & $-117^{\circ} 57.916^{\prime}$ & 2016 & 25 \\
\hline Owens Lake & T8-W & $36^{\circ} 22.522^{\prime}$ & $-117^{\circ} 57.256^{\prime}$ & 2018 & 144 \\
\hline
\end{tabular}

TABLE A.1: Sites visited in 2016 were consistently sampled with a lower resolution but more sites were covered. The second campaign focused on fewer experiments but with a much higher resolution. 


\section{A.2 Site description Badwater Basin (1) ${ }^{1}$}
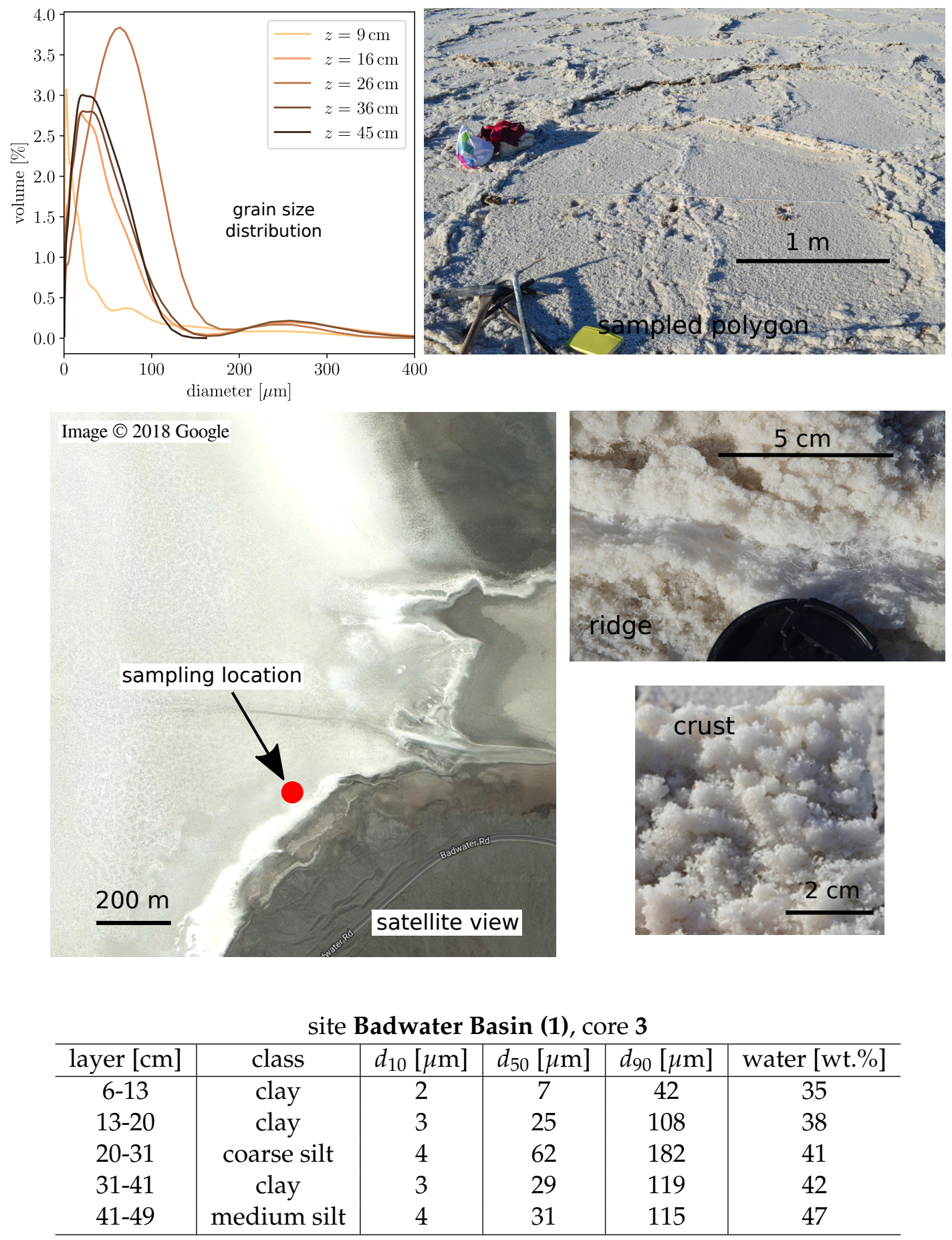

TABLE A.2: Particle size and water content for site Badwater Basin (1). Layer is specified as distance from surface. 


\section{A.3 Site description Badwater Basin (2) ${ }^{2}$}
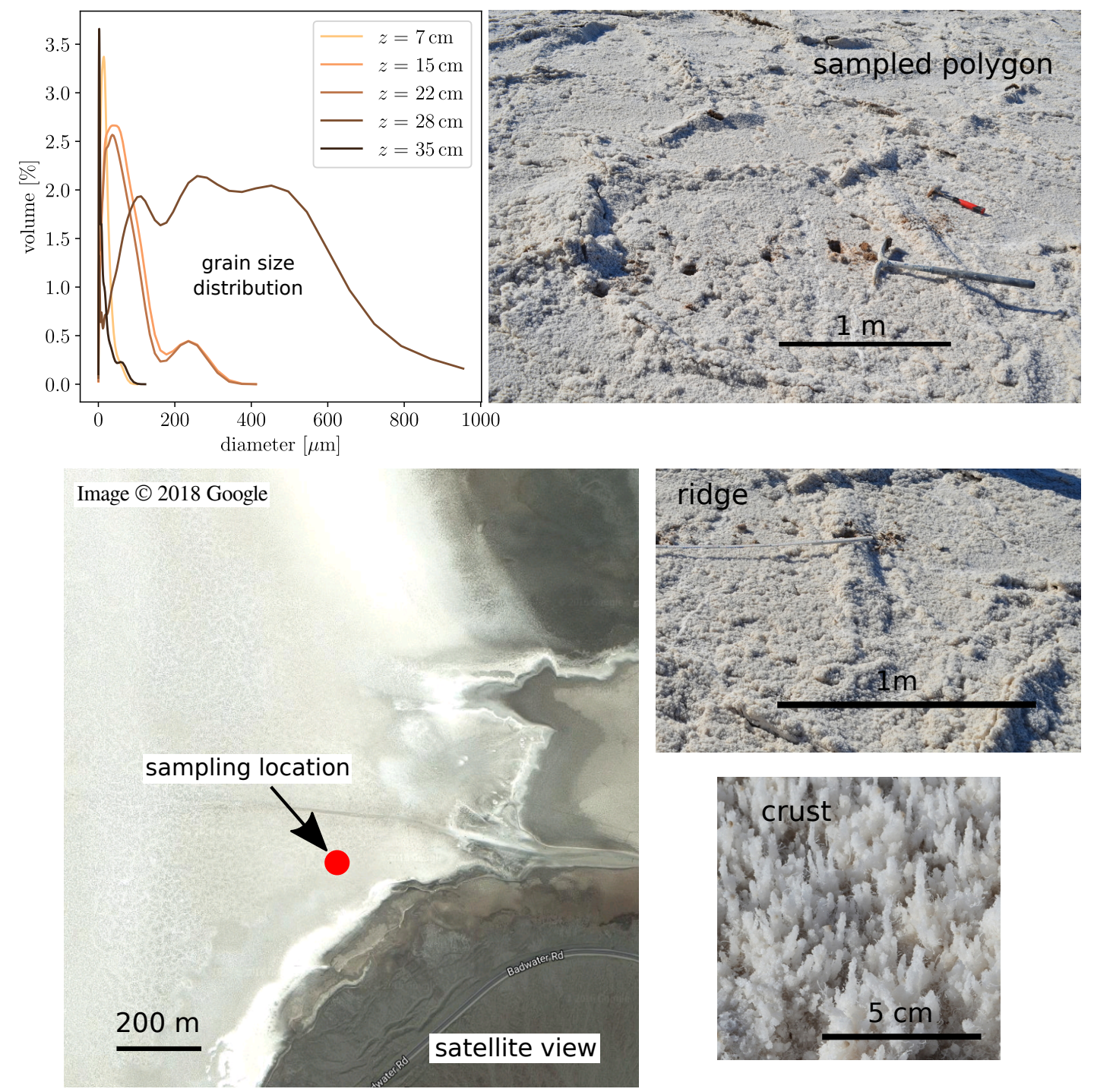

site Badwater Basin (1), core 3

\begin{tabular}{c|c|c|c|c|c}
\hline layer $[\mathrm{cm}]$ & class & $d_{10}[\mu \mathrm{m}]$ & $d_{50}[\mu \mathrm{m}]$ & $d_{90}[\mu \mathrm{m}]$ & water [wt.\%] \\
\hline $3-11$ & clay & 2 & 13 & 45 & 33 \\
$11-19$ & medium silt & 4 & 41 & 178 & 37 \\
$19-25$ & clay & 3 & 33 & 159 & 36 \\
$25-32$ & clay & 2 & 112 & 849 & 38 \\
$32-38$ & clay & 1 & 5 & 25 & 73 \\
\hline
\end{tabular}

TABLE A.3: Particle size and water content for site Badwater Basin (1). Layer is specified as distance from surface. 


\section{A.4 Site description Badwater Basin (3)}
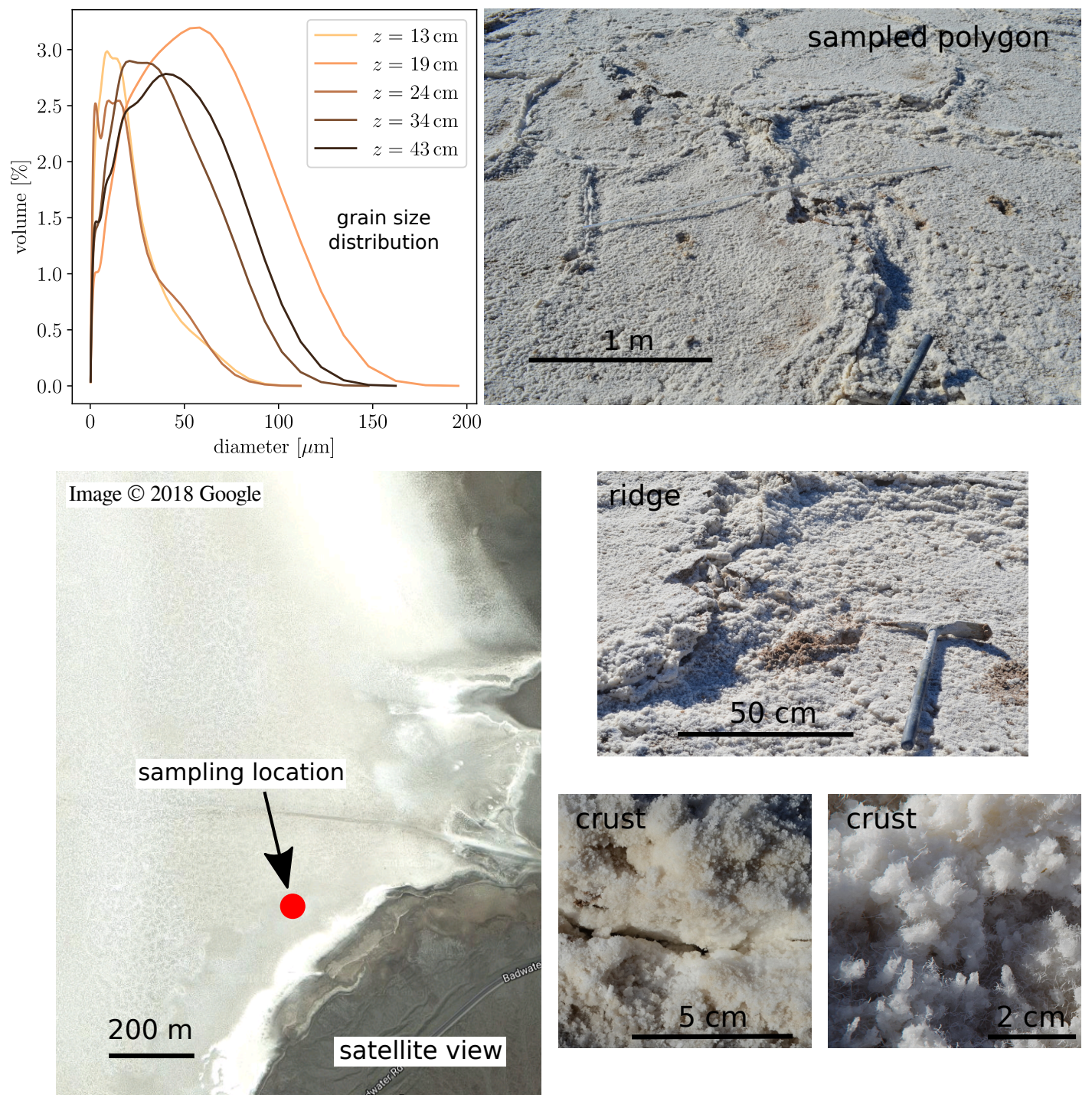

site Badwater Basin (1), core 3

\begin{tabular}{c|c|c|c|c|c}
\hline layer [cm] & class & $d_{10}[\mu \mathrm{m}]$ & $d_{50}[\mu \mathrm{m}]$ & $d_{90}[\mu \mathrm{m}]$ & water [wt.\%] \\
\hline $10-16$ & clay & 2 & 13 & 49 & 32 \\
$16-22$ & coarse silt & 3 & 45 & 157 & 32 \\
$22-29$ & clay & 2 & 10 & 49 & 30 \\
$29-39$ & clay & 3 & 27 & 105 & 31 \\
$39-47$ & clay & 3 & 29 & 124 & 35 \\
\hline
\end{tabular}

TABLE A.4: Particle size and water content for site Badwater Basin (1). Layer is specified as distance from surface. 


\section{A.5 Site description Badwater Basin (4)}
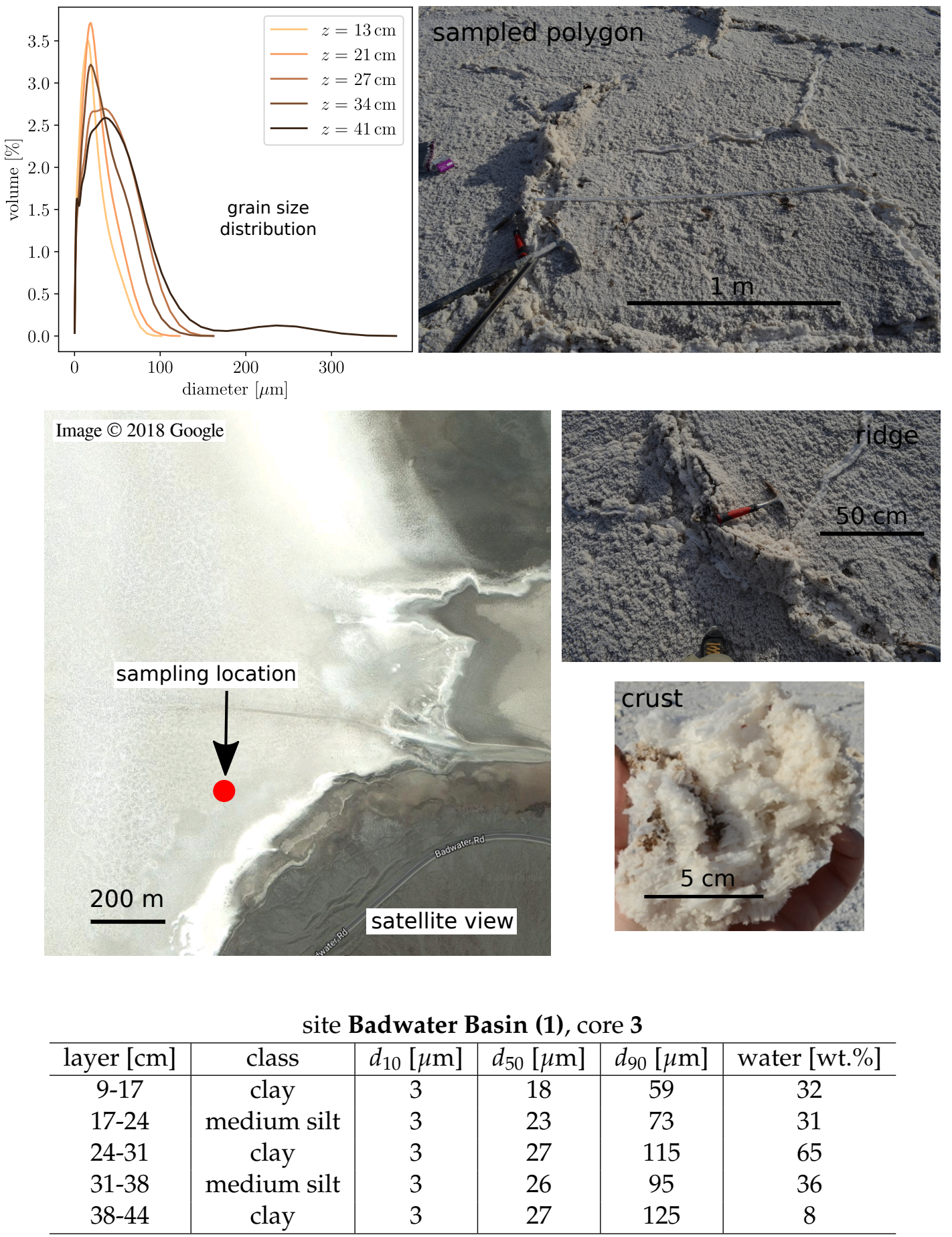

TABLE A.5: Particle size and water content for site Badwater Basin (1). Layer is specified as distance from surface. 


\section{A.6 Site description Badwater Basin (5)}
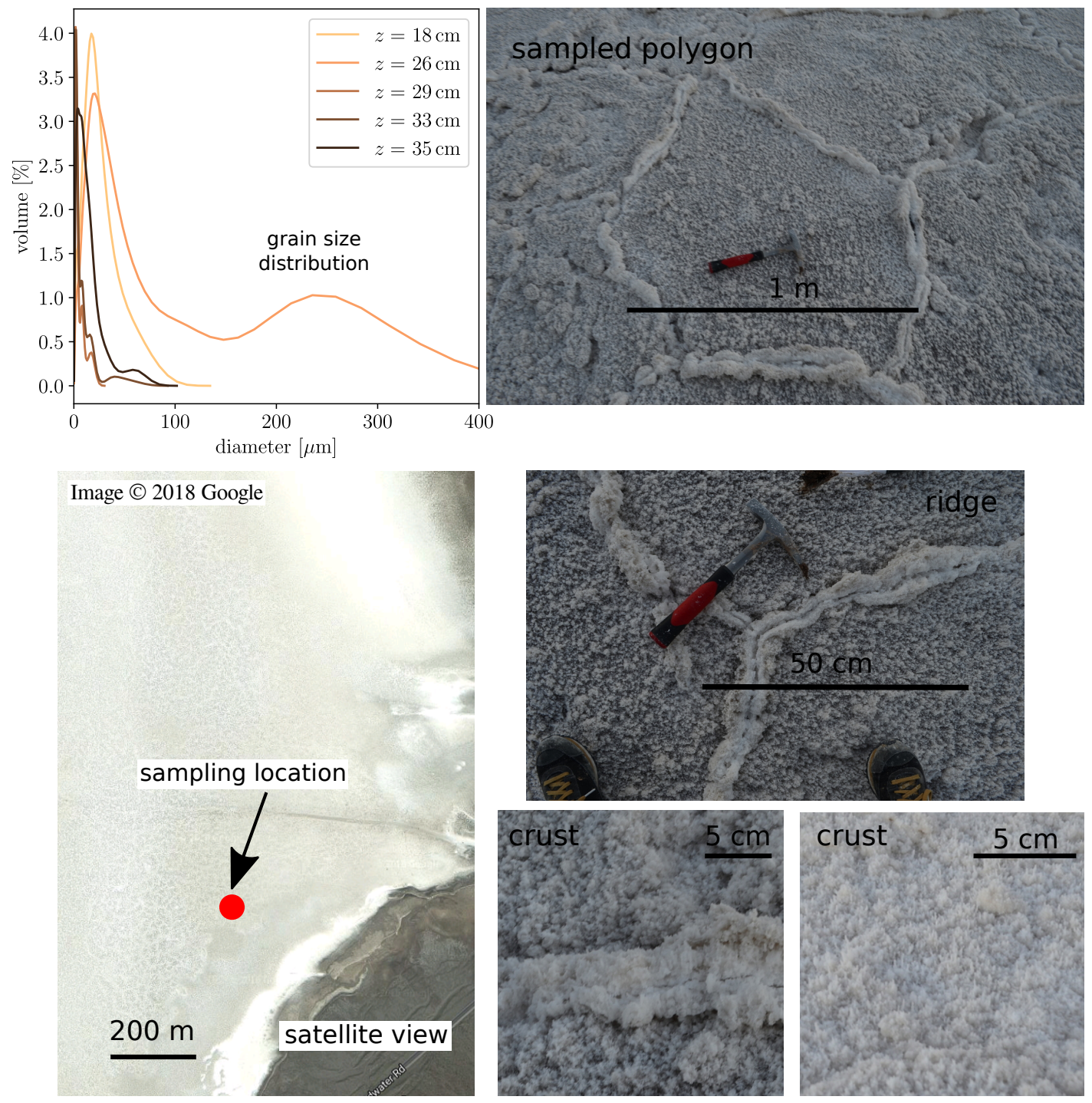

site Badwater Basin (1), core 3

\begin{tabular}{c|c|c|c|c|c}
\hline layer $[\mathrm{cm}]$ & class & $d_{10}[\mu \mathrm{m}]$ & $d_{50}[\mu \mathrm{m}]$ & $d_{90}[\mu \mathrm{m}]$ & water [wt.\%] \\
\hline $14-22$ & medium silt & 3 & 22 & 67 & 19 \\
$22-28$ & medium silt & 4 & 35 & 210 & 21 \\
$28-31$ & clay & 1 & 3 & 9 & 26 \\
$31-34$ & clay & 1 & 3 & 14 & 30 \\
$34-36$ & clay & 2 & 8 & 32 & 28 \\
\hline
\end{tabular}

TABLE A.6: Particle size and water content for site Badwater Basin (1). Layer is specified as distance from surface. 


\section{A.7 Site description Bristol Playa ${ }^{6}$}
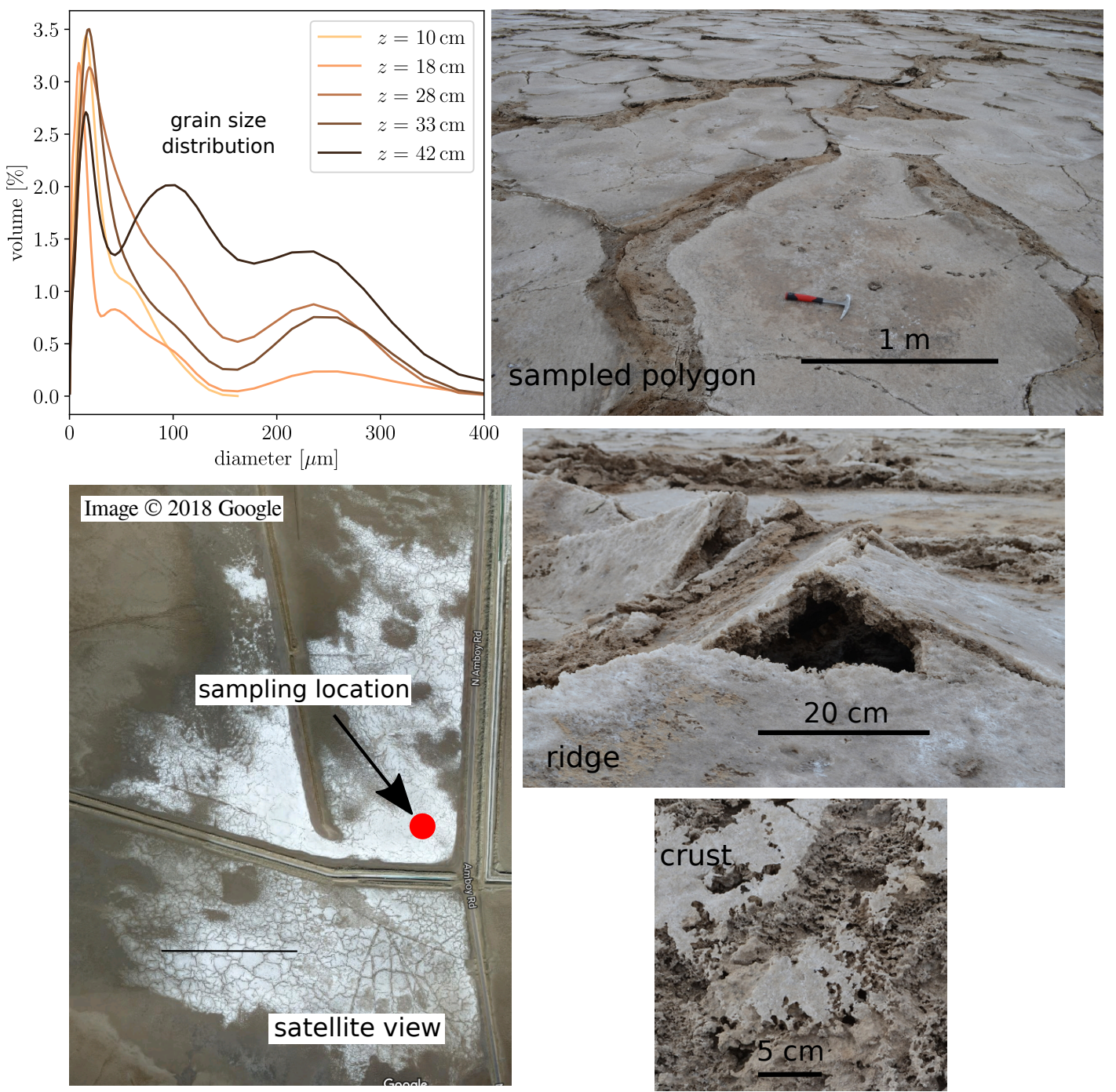

site Bristol Playa, core 3

\begin{tabular}{c|c|c|c|c|c}
\hline layer $[\mathrm{cm}]$ & class & $d_{10}[\mu \mathrm{m}]$ & $d_{50}[\mu \mathrm{m}]$ & $d_{90}[\mu \mathrm{m}]$ & water [wt.\%] \\
\hline $6-14$ & clay & 3 & 20 & 79 & 25 \\
$14-23$ & clay & 2 & 13 & 71 & 14 \\
$23-31$ & medium silt & 4 & 36 & 191 & 24 \\
$31-38$ & medium silt & 4 & 29 & 137 & 33 \\
$38-46$ & medium silt & 5 & 39 & 328 & 16 \\
\hline
\end{tabular}

TABLE A.7: Particle size and water content for site Bristol Playa. Layer is specified as distance from surface. 


\section{A.8 Site description Owens Lake T10-3}
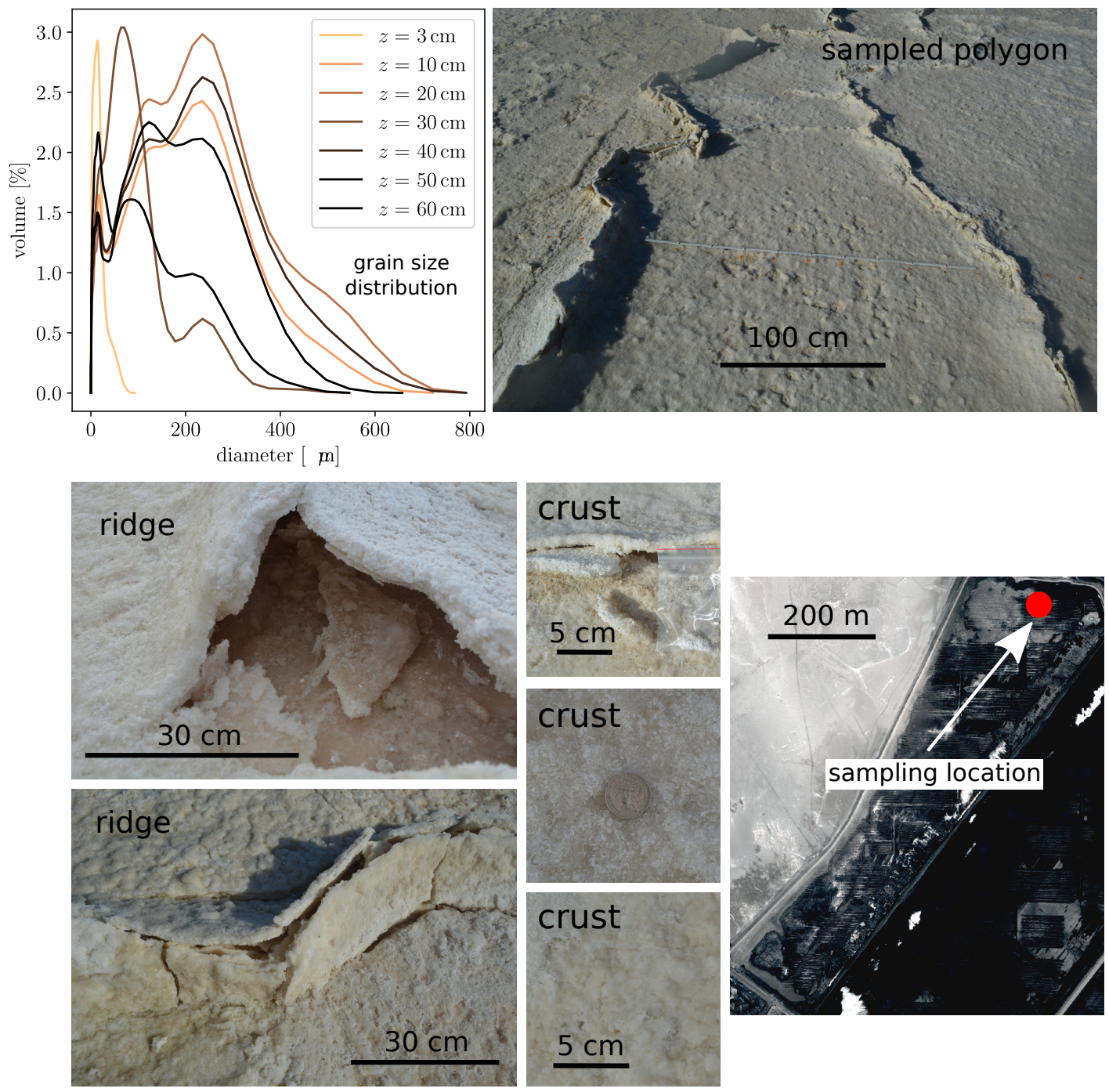

T10-3, core 8

\begin{tabular}{c|c|c|c|c|c}
\hline layer $[\mathrm{cm}]$ & class & $d_{10}[\mu \mathrm{m}]$ & $d_{50}[\mu \mathrm{m}]$ & $d_{90}[\mu \mathrm{m}]$ & water [wt.\%] \\
\hline $0-7$ & clay & 1 & 10 & 39 & 49 \\
$7-15$ & clay & 3 & 52 & 493 & 44 \\
$15-25$ & fine sand & 4 & 119 & 582 & 48 \\
$25-35$ & very fine sand & 3 & 48 & 210 & 36 \\
$35-45$ & clay & 3 & 80 & 546 & 58 \\
$45-55$ & clay & 2 & 52 & 458 & 55 \\
$55-65$ & clay & 2 & 27 & 254 & 62 \\
\hline
\end{tabular}

TABLE A.8: Particle size and water content for site T10-3. Layer is specified as distance from surface.

\footnotetext{
${ }^{7}$ Satellite image courtesy Grace Holder, LADWP
} 


\section{A.9 Site description Owens Lake T16}
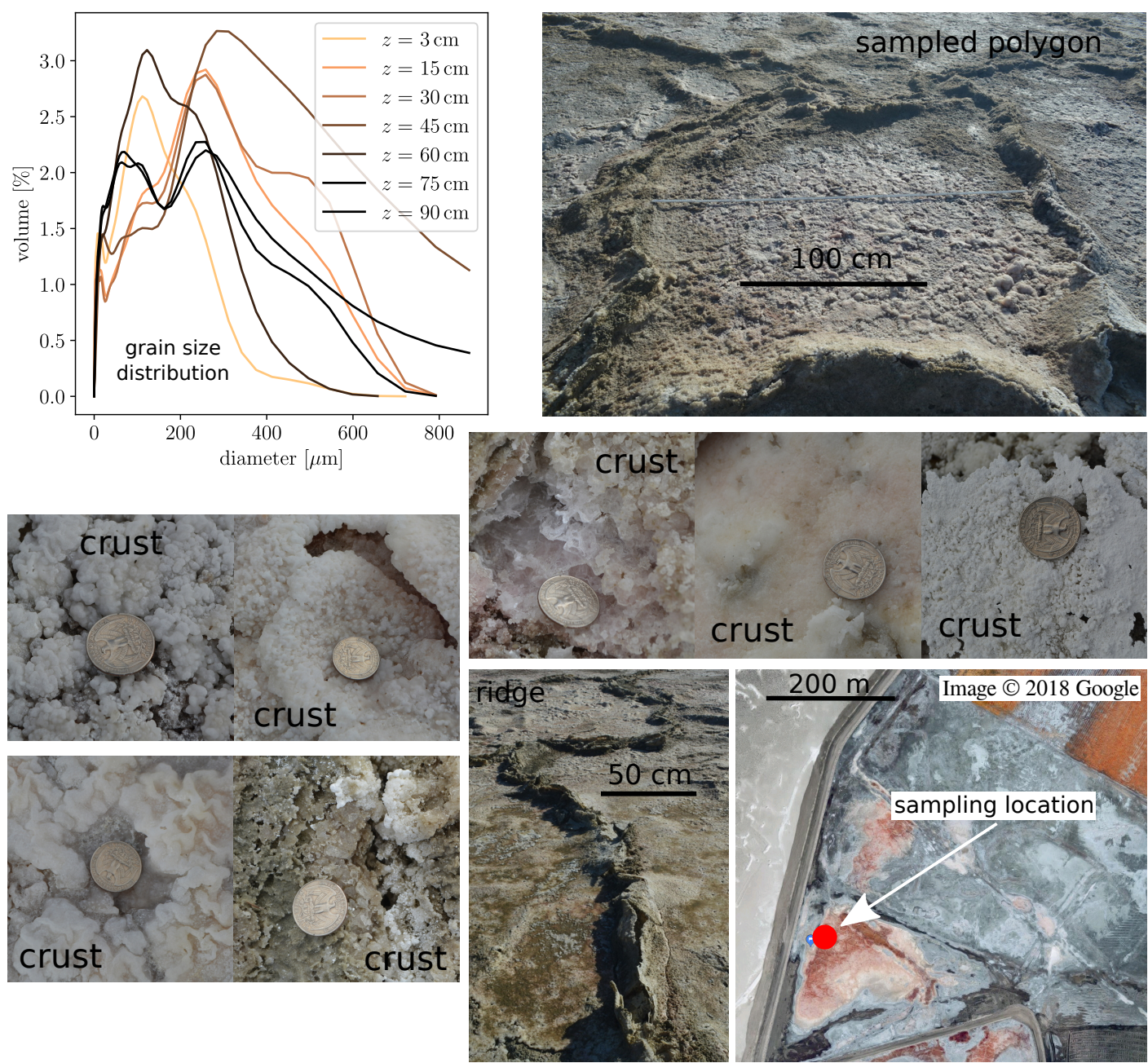

site $\mathbf{T} 16$, core 11

\begin{tabular}{c|c|c|c|c|c}
\hline layer $[\mathrm{cm}]$ & class & $d_{10}[\mu \mathrm{m}]$ & $d_{50}[\mu \mathrm{m}]$ & $d_{90}[\mu \mathrm{m}]$ & water [wt.\%] \\
\hline $0-9$ & clay & 3 & 56 & 368 & 67 \\
$9-23$ & clay & 3 & 102 & 682 & 86 \\
$23-38$ & clay & 3 & 117 & 772 & 98 \\
$38-53$ & medium sand & 15 & 247 & 1096 & 48 \\
$53-68$ & very fine sand & 8 & 121 & 473 & 114 \\
$68-83$ & very fine sand & 8 & 108 & 702 & 149 \\
$83-98$ & very fine sand & 6 & 91 & 581 & 161 \\
\hline
\end{tabular}

TABLE A.9: Particle size and water content for site T16. Layer is specified as distance from surface. 


\section{A.10 Site description Owens Lake T2-4}
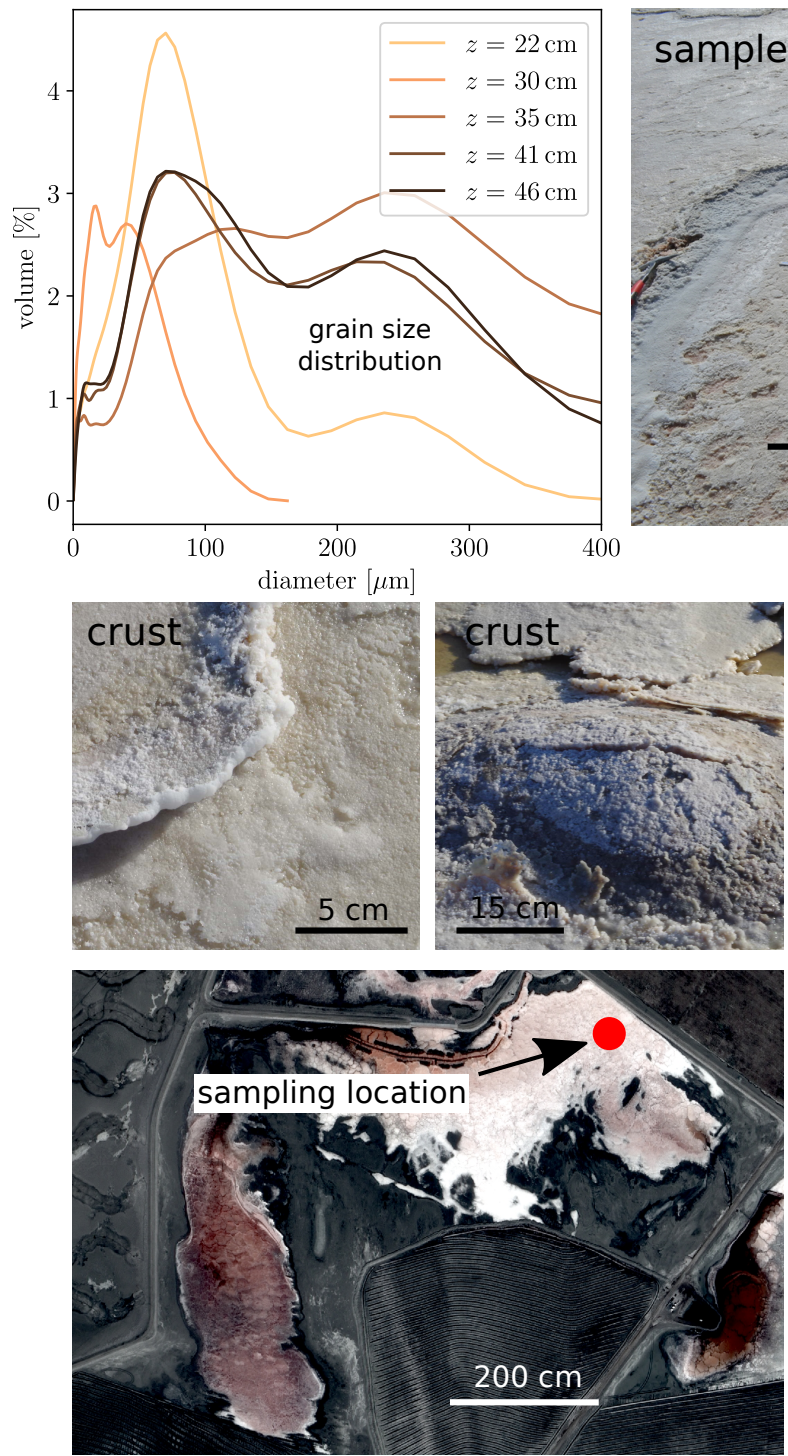

site $\mathbf{T} 2-4$, core 3

\begin{tabular}{c|c|c|c|c|c}
\hline layer $[\mathrm{cm}]$ & class & $d_{10}[\mu \mathrm{m}]$ & $d_{50}[\mu \mathrm{m}]$ & $d_{90}[\mu \mathrm{m}]$ & water [wt.\%] \\
\hline $18-26$ & very fine sand & 5 & 87 & 232 & 25 \\
$26-32$ & medium silt & 3 & 28 & 112 & 43 \\
$32-38$ & very fine sand & 6 & 179 & 729 & 19 \\
$38-44$ & very fine sand & 7 & 124 & 529 & 20 \\
$44-48$ & very fine sand & 9 & 126 & 511 & 18 \\
\hline
\end{tabular}

TABLE A.10: Particle size and water content for site T2-4. Layer is specified as distance from surface.

\footnotetext{
${ }^{9}$ Satellite image courtesy Grace Holder, LADWP
} 


\section{A.11 Site description Owens Lake T2-5 (1)}
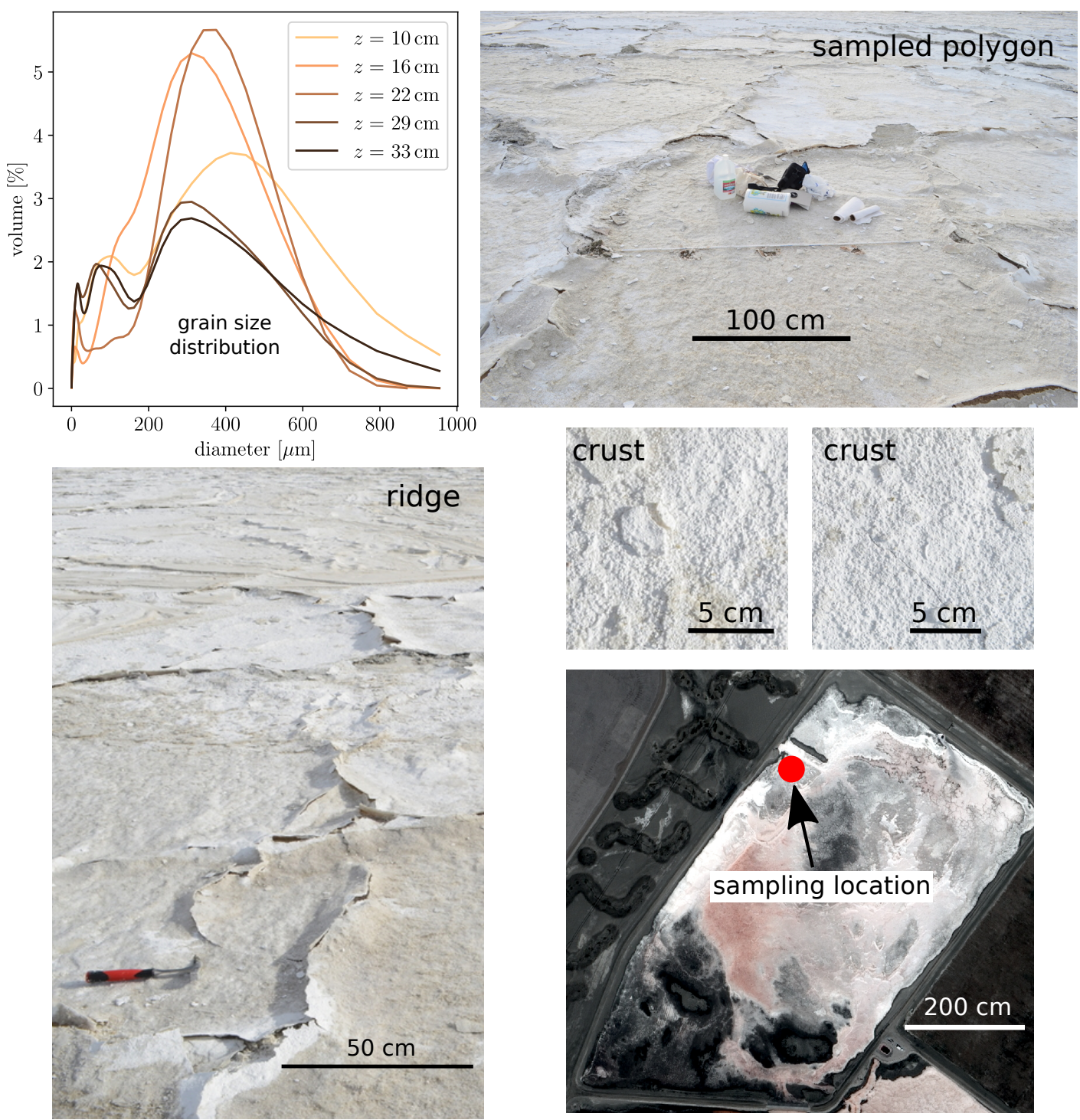

site T2-5 (1), core 3

\begin{tabular}{c|c|c|c|c|c}
\hline layer $[\mathrm{cm}]$ & class & $d_{10}[\mu \mathrm{m}]$ & $d_{50}[\mu \mathrm{m}]$ & $d_{90}[\mu \mathrm{m}]$ & water [wt.\%] \\
\hline $7-13$ & medium sand & 21 & 300 & 1113 & 47 \\
$13-19$ & medium sand & 17 & 422 & 927 & 26 \\
$19-26$ & medium sand & 8 & 422 & 942 & 15 \\
$26-31$ & medium sand & 10 & 126 & 814 & 15 \\
$31-35$ & medium sand & 8 & 136 & 874 & 21 \\
\hline
\end{tabular}

TABLE A.11: Particle size and water content for site T2-5 (1). Layer is specified as distance from surface. 


\section{A.12 Site description Owens Lake T2-5 (2) ${ }^{11}$}
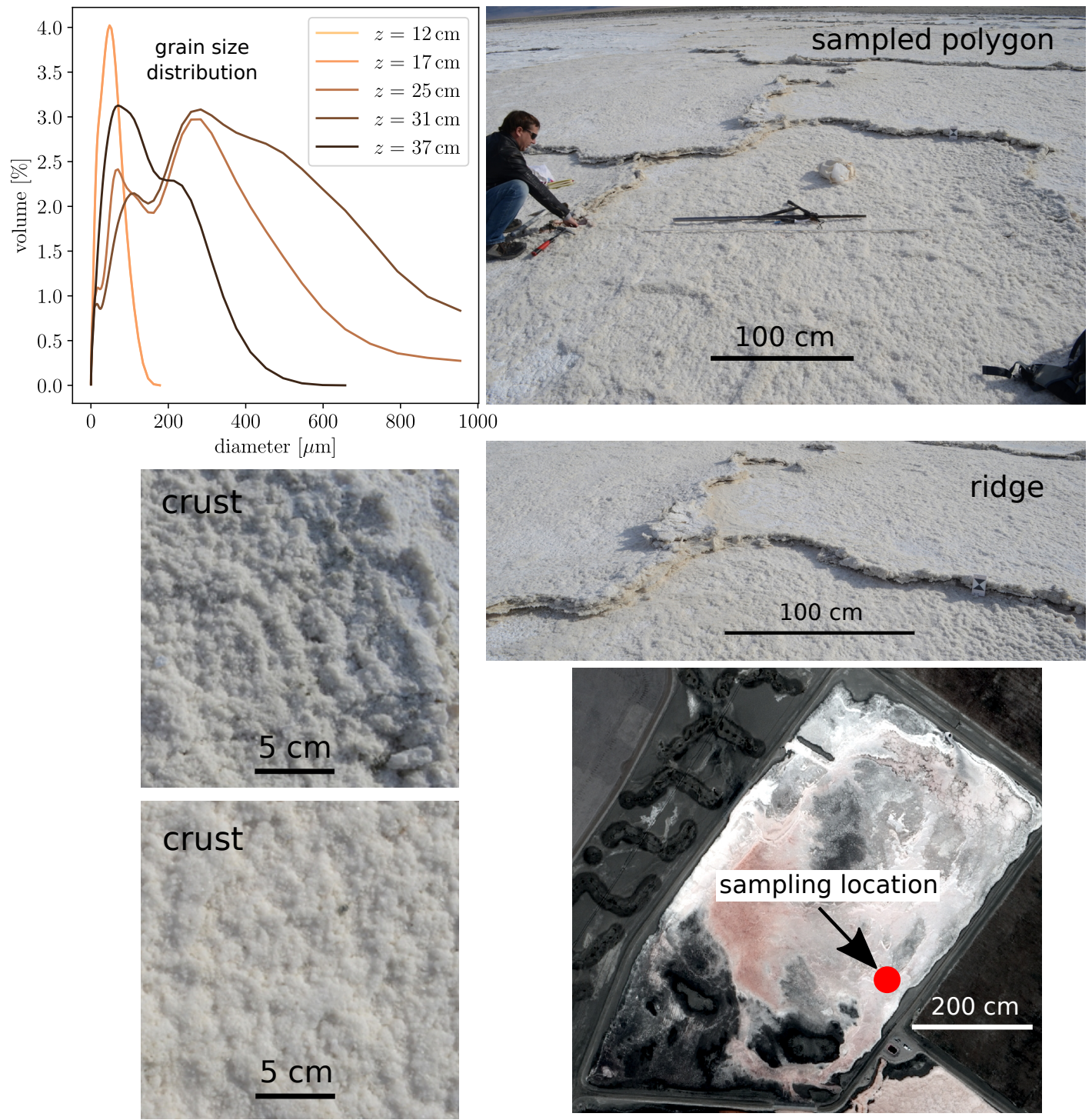

site T2-5 (2), core 3

\begin{tabular}{c|c|c|c|c|c}
\hline layer $[\mathrm{cm}]$ & class & $d_{10}[\mu \mathrm{m}]$ & $d_{50}[\mu \mathrm{m}]$ & $d_{90}[\mu \mathrm{m}]$ & water [wt.\%] \\
\hline $10-15$ & coarse silt & 8 & 53 & 145 & 28 \\
$15-21$ & nan & 11 & 126 & 775 & 16 \\
$21-28$ & medium sand & 8 & 156 & 762 & 28 \\
$28-34$ & medium sand & 13 & 264 & 1096 & 21 \\
$34-40$ & very fine sand & 14 & 117 & 446 & 18 \\
\hline
\end{tabular}

TABLE A.12: Particle size and water content for site T2-5 (2). Layer is specified as distance from surface. 


\section{A.13 Site description Owens Lake T2-5 (3) ${ }^{12}$}
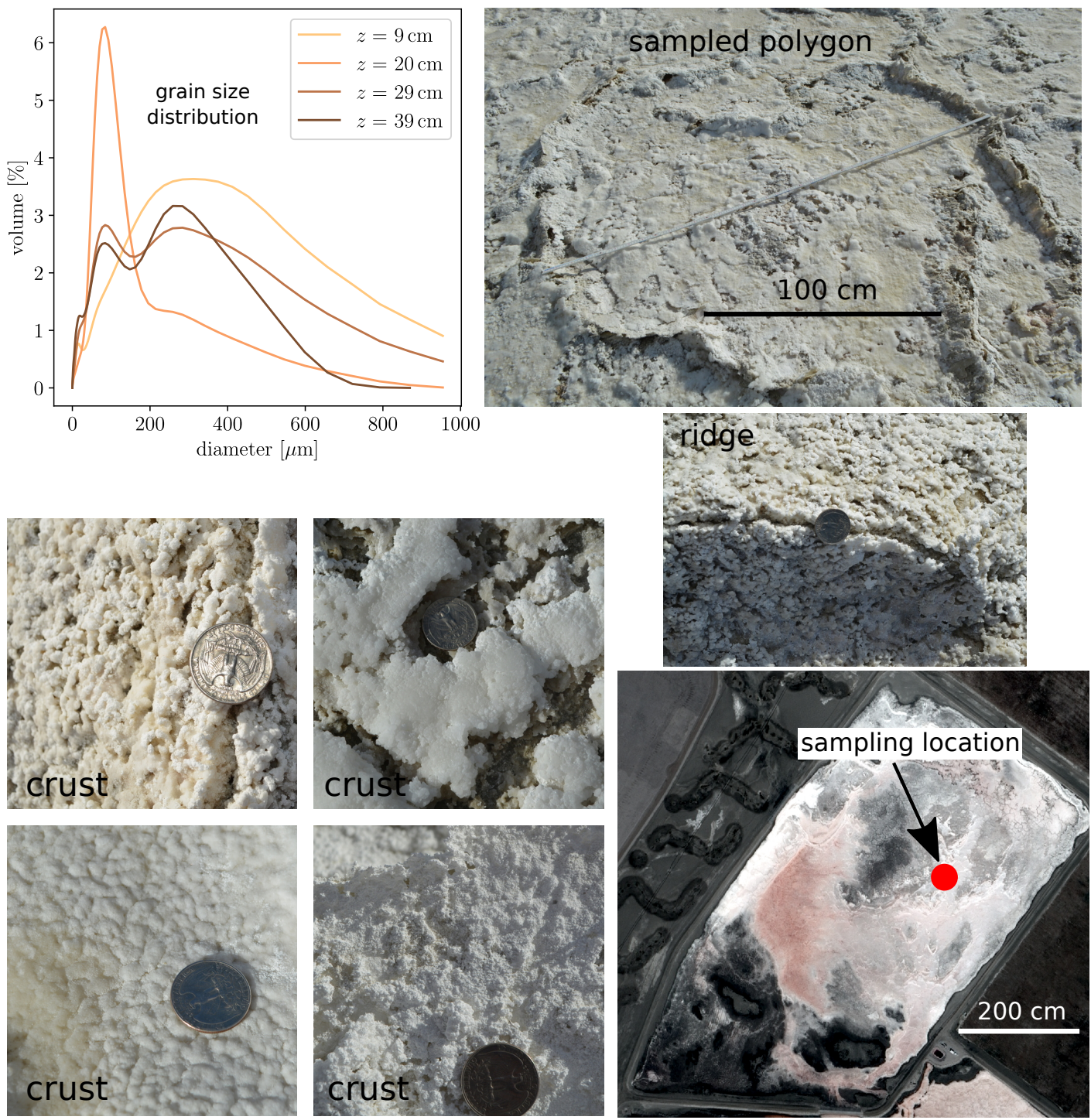

site T2-5 (3), core 7

\begin{tabular}{c|c|c|c|c|c}
\hline layer $[\mathrm{cm}]$ & class & $d_{10}[\mu \mathrm{m}]$ & $d_{50}[\mu \mathrm{m}]$ & $d_{90}[\mu \mathrm{m}]$ & water [wt.\%] \\
\hline $4-14$ & medium sand & 24 & 381 & 1138 & 24 \\
$14-25$ & very fine sand & 46 & 163 & 481 & 27 \\
$25-34$ & very fine sand & 21 & 213 & 935 & 31 \\
$34-44$ & medium sand & 11 & 167 & 717 & 30 \\
\hline
\end{tabular}

TABLE A.13: Particle size and water content for site T2-5 (3). Layer is specified as distance from surface. 


\section{A.14 Site description Owens Lake T25-3 (1) ${ }^{13}$}
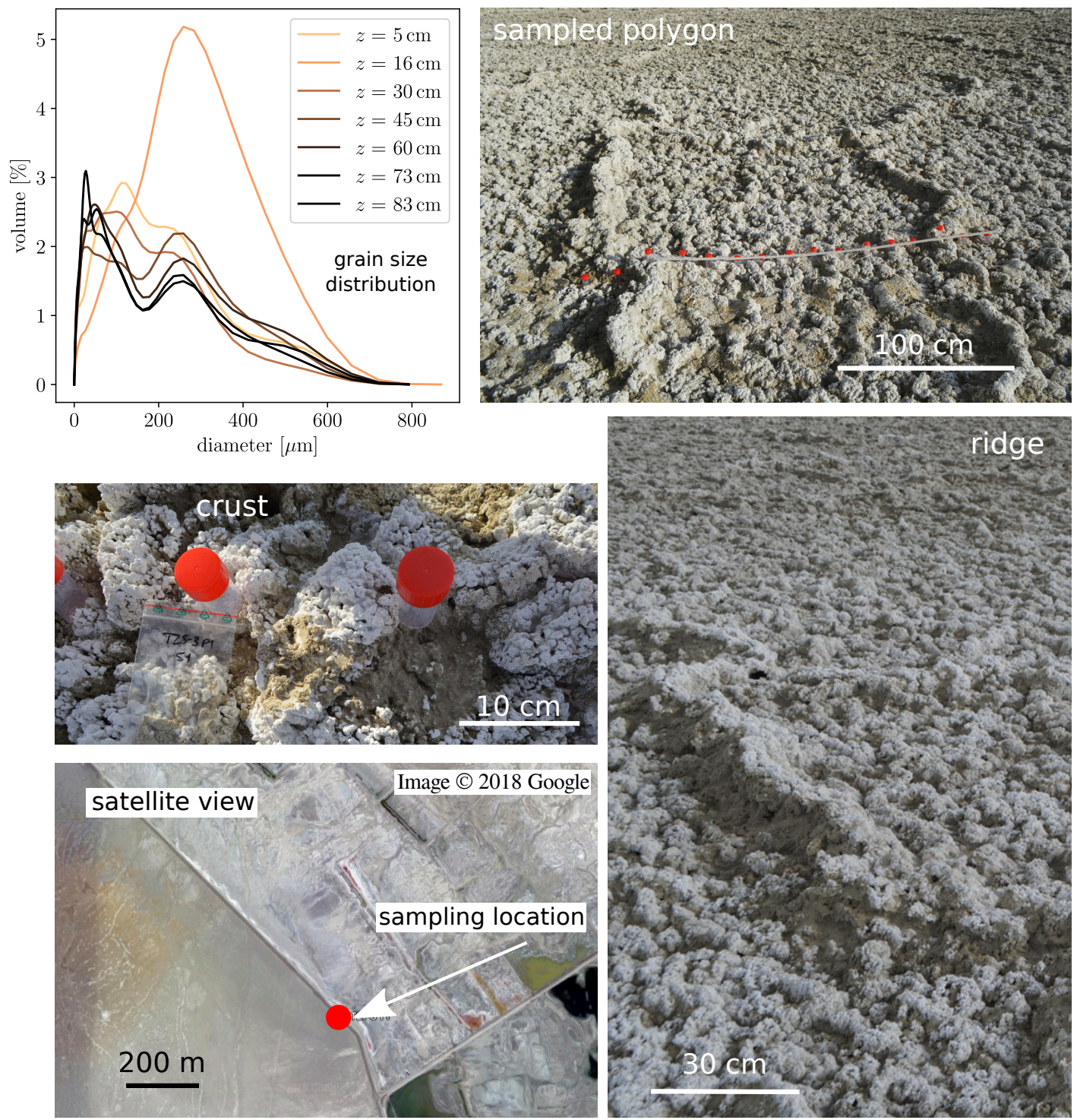

site T25-3 (1), core 8

\begin{tabular}{c|c|c|c|c|c}
\hline layer $[\mathrm{cm}]$ & class & $d_{10}[\mu \mathrm{m}]$ & $d_{50}[\mu \mathrm{m}]$ & $d_{90}[\mu \mathrm{m}]$ & water [wt.\%] \\
\hline $0-11$ & very fine sand & 6 & 117 & 506 & 20 \\
$11-23$ & medium sand & 19 & 339 & 782 & 22 \\
$23-38$ & very fine sand & 8 & 87 & 442 & 116 \\
$38-53$ & medium silt & 6 & 69 & 533 & 80 \\
$53-67$ & coarse silt & 8 & 81 & 525 & 148 \\
$67-78$ & medium silt & 8 & 62 & 455 & 137 \\
$78-88$ & medium silt & 6 & 62 & 445 & 152 \\
\hline
\end{tabular}

TABLE A.14: Particle size and water content for site T25-3 (1). Layer is specified as distance from surface. 


\section{A.15 Site description Owens Lake T25-3 (2) ${ }^{14}$}
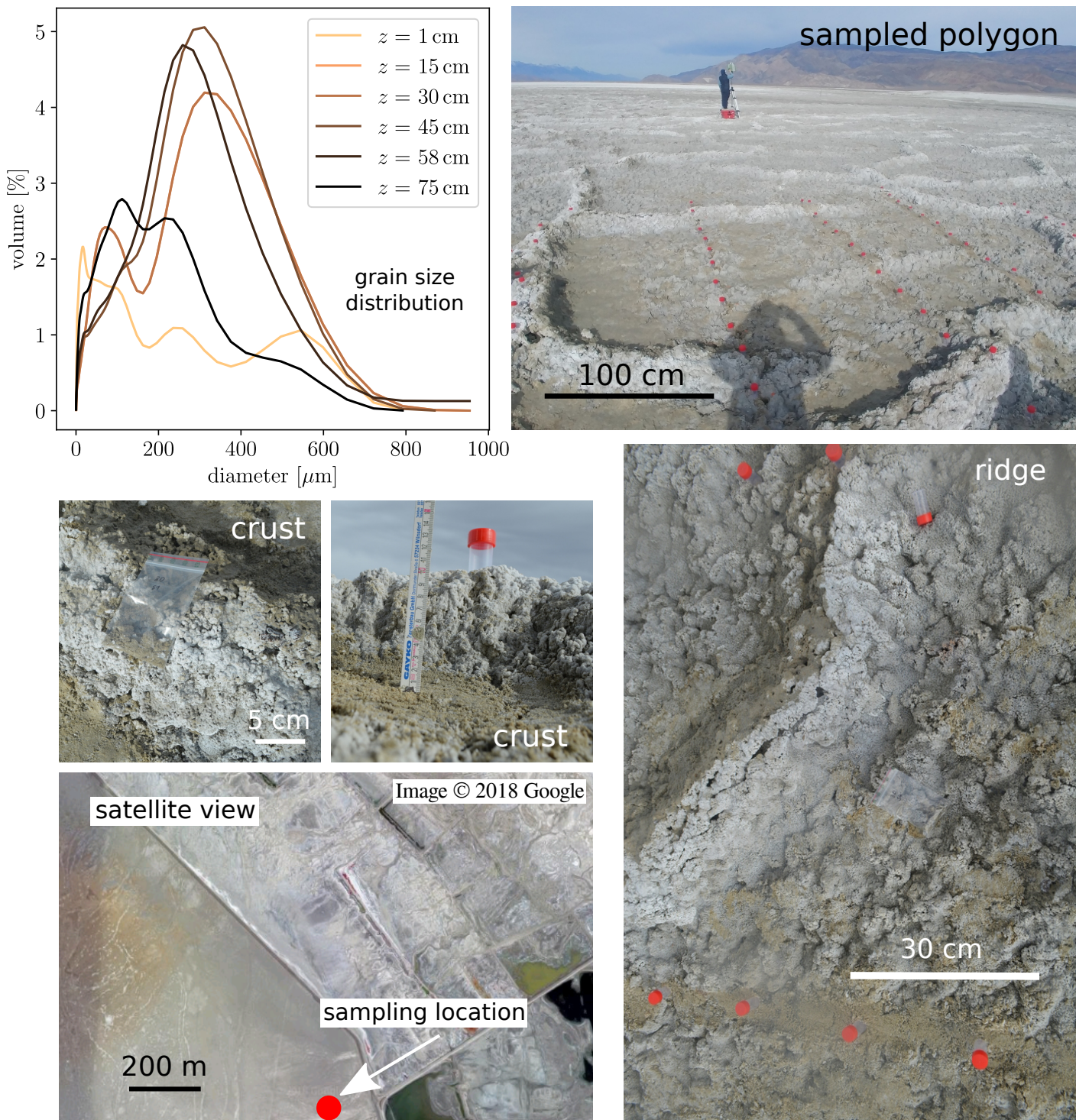

site T25-3 (2), core 25

\begin{tabular}{c|c|c|c|c|c}
\hline layer $[\mathrm{cm}]$ & class & $d_{10}[\mu \mathrm{m}]$ & $d_{50}[\mu \mathrm{m}]$ & $d_{90}[\mu \mathrm{m}]$ & water [wt.\%] \\
\hline $1-9$ & clay & 4 & 40 & 451 & 42 \\
$9-27$ & medium sand & 21 & 224 & 849 & 36 \\
$27-39$ & 0 & 0 & 0 & 0 & 47 \\
$39-54$ & medium sand & 16 & 343 & 842 & 50 \\
$54-60$ & medium sand & 13 & 292 & 763 & 67 \\
$60-80$ & very fine sand & 8 & 117 & 519 & 214 \\
\hline
\end{tabular}

TABLE A.15: Particle size and water content for site T25-3 (2). Layer is specified as distance from surface.

\footnotetext{
${ }^{14}$ No exact GPS coordinates available. Location in close vicinity to T25-3 (1)
} 


\section{A.16 Site description Owens Lake T27-A (1) ${ }^{15}$}
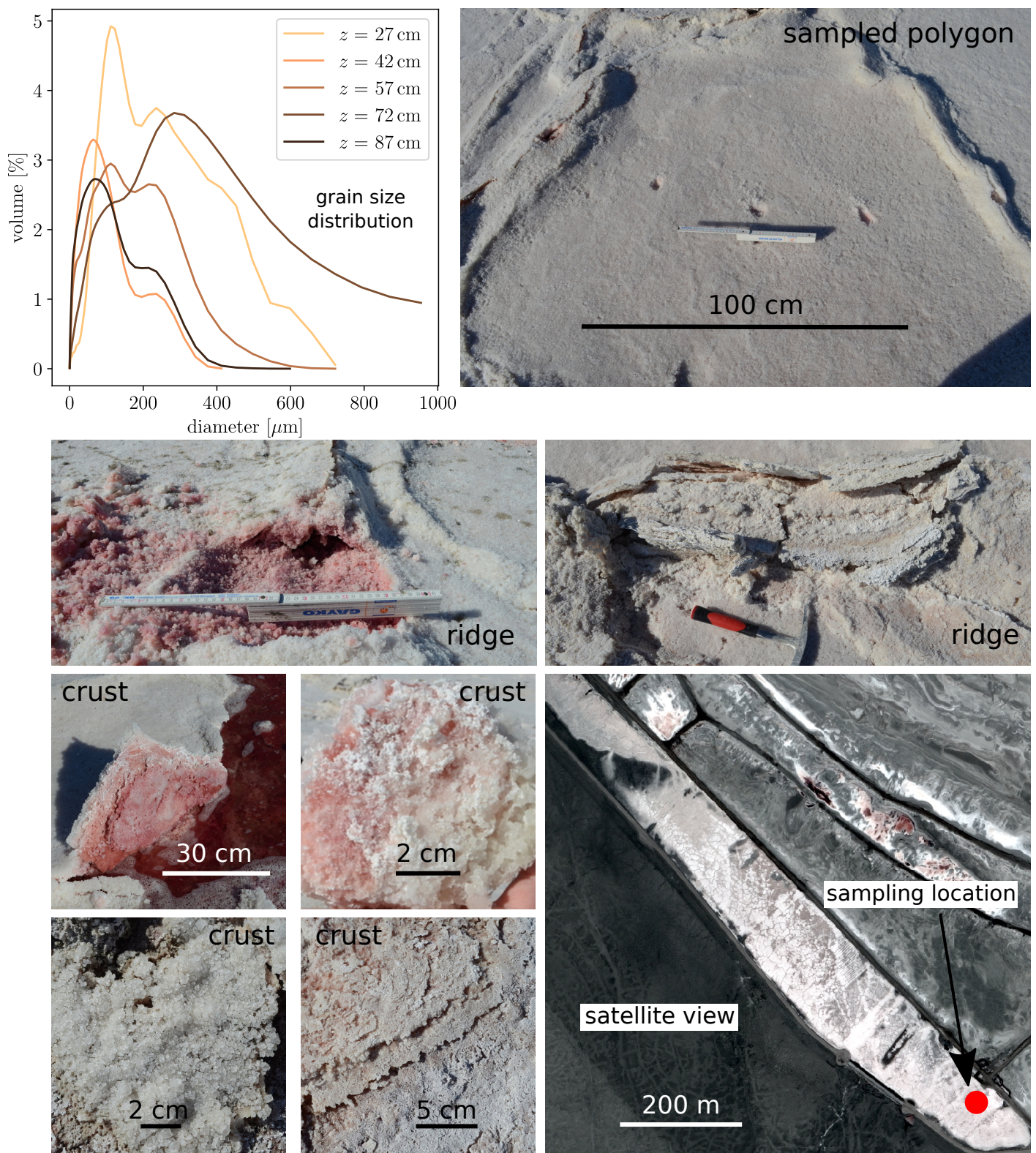

site T27-A (1), core 3

\begin{tabular}{c|c|c|c|c|c}
\hline layer $[\mathrm{cm}]$ & class & $d_{10}[\mu \mathrm{m}]$ & $d_{50}[\mu \mathrm{m}]$ & $d_{90}[\mu \mathrm{m}]$ & water [wt.\%] \\
\hline $20-35$ & very fine sand & 81 & 267 & 758 & 24 \\
$35-50$ & very fine sand & 10 & 77 & 273 & 38 \\
$50-65$ & very fine sand & 10 & 122 & 487 & 101 \\
$65-80$ & medium sand & 32 & 319 & 1043 & 128 \\
$80-95$ & very fine sand & 8 & 71 & 321 & 125 \\
\hline
\end{tabular}

TABLE A.16: Particle size and water content for site T27-A (1). Layer is specified as distance from surface.

${ }^{15}$ Satellite image courtesy Grace Holder, LADWP 


\section{A.17 Site description Owens Lake T27-A (2) ${ }^{16}$}
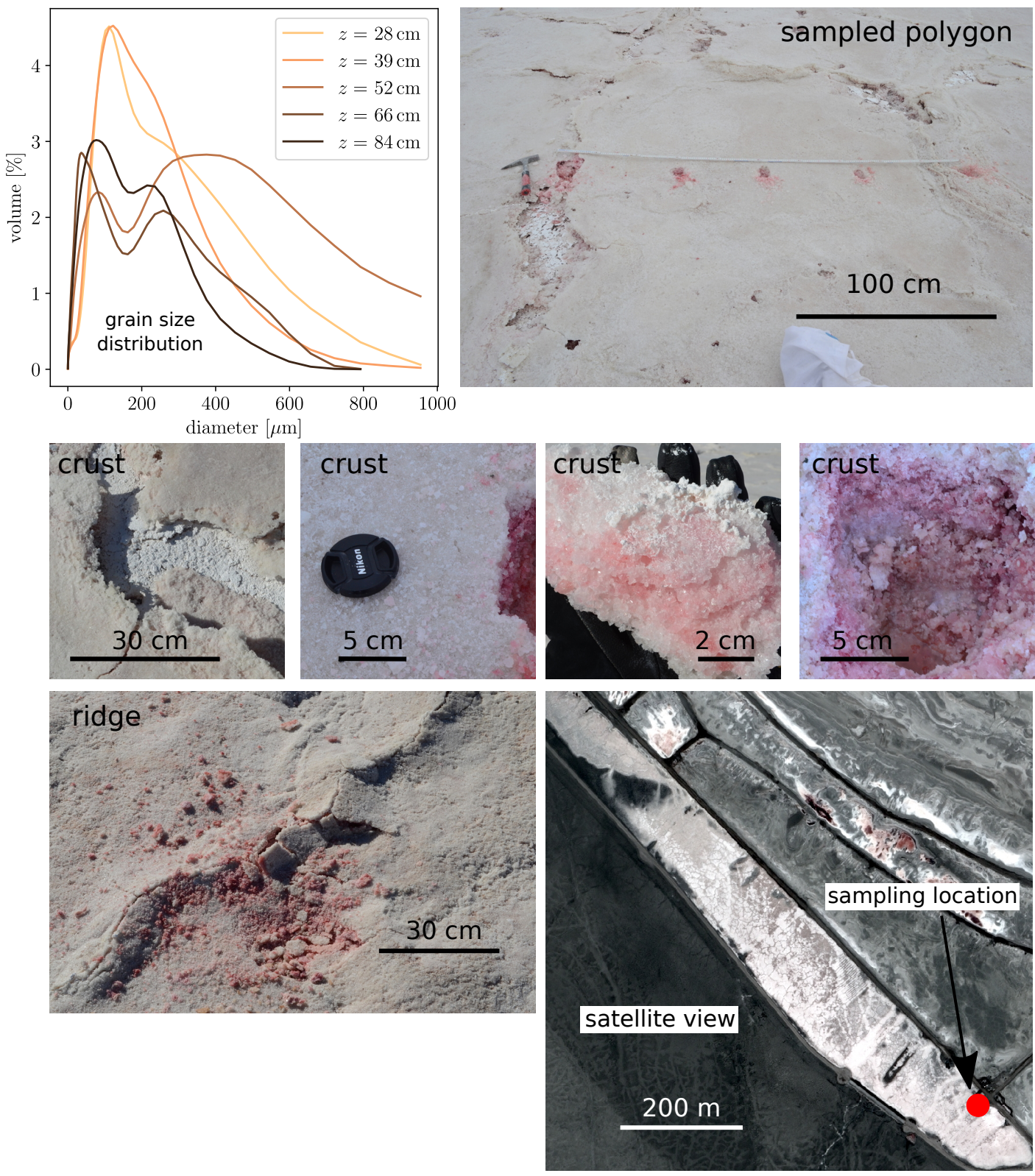

site T27-A (2), core 3

\begin{tabular}{c|c|c|c|c|c}
\hline layer $[\mathrm{cm}]$ & class & $d_{10}[\mu \mathrm{m}]$ & $d_{50}[\mu \mathrm{m}]$ & $d_{90}[\mu \mathrm{m}]$ & water [wt.\%] \\
\hline $23-34$ & very fine sand & 44 & 247 & 790 & 16 \\
$34-46$ & very fine sand & 49 & 236 & 626 & 15 \\
$46-59$ & medium sand & 27 & 232 & 1107 & 45 \\
$59-75$ & coarse silt & 14 & 97 & 589 & 72 \\
$75-93$ & very fine sand & 15 & 123 & 492 & 77
\end{tabular}

TABLE A.17: Particle size and water content for site T27-A (2). Layer is specified as distance from surface.

\footnotetext{
${ }^{16}$ Satellite image courtesy Grace Holder, LADWP
} 


\section{A.18 Site description Owens Lake T27-A (3) ${ }^{17}$}
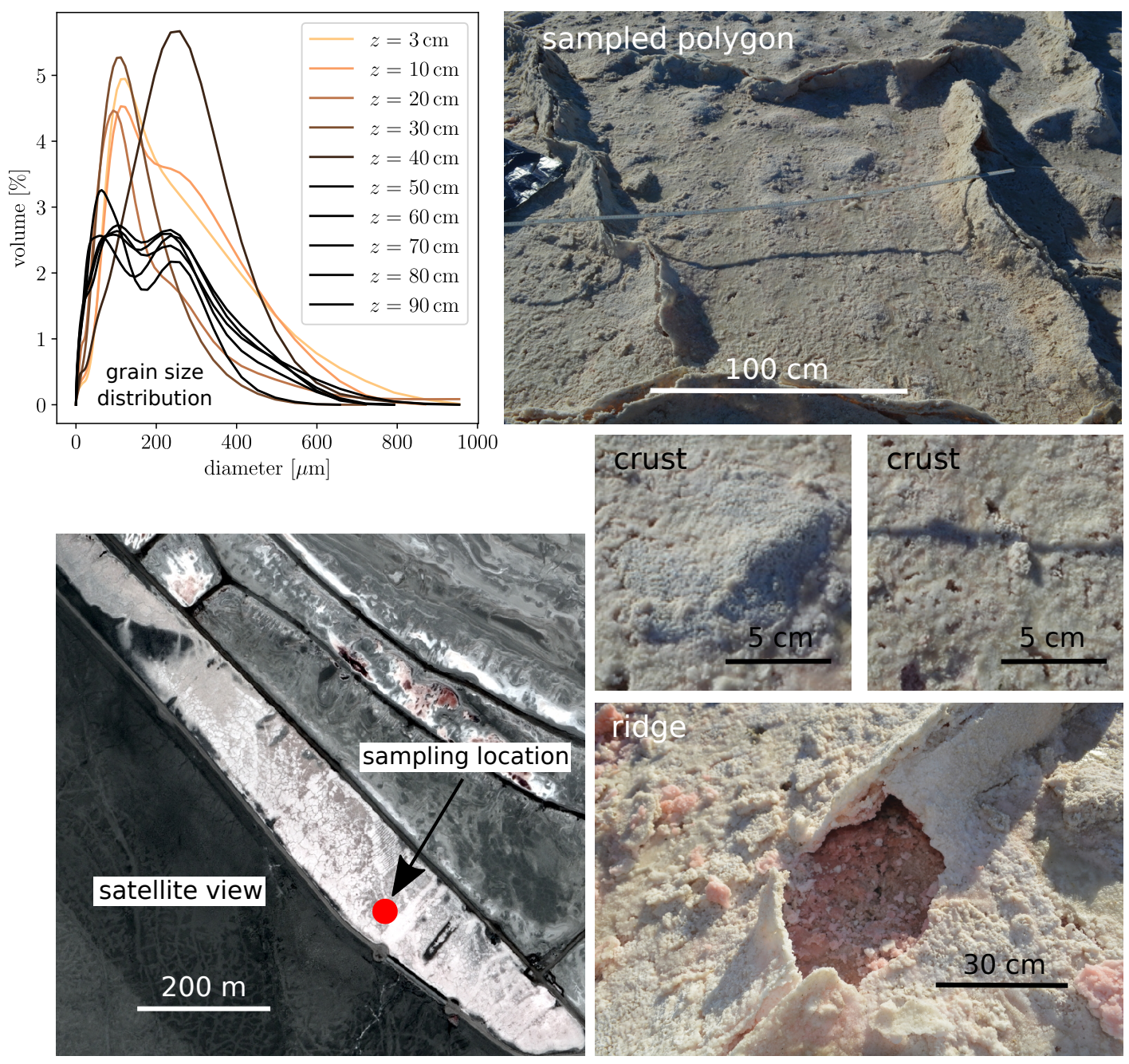

site T27-A (3), core 7

\begin{tabular}{c|c|c|c|c|c}
\hline layer $[\mathrm{cm}]$ & class & $d_{10}[\mu \mathrm{m}]$ & $d_{50}[\mu \mathrm{m}]$ & $d_{90}[\mu \mathrm{m}]$ & water [wt.\%] \\
\hline $0-7$ & very fine sand & 65 & 257 & 725 & 24 \\
$7-15$ & very fine sand & 44 & 257 & 716 & 19 \\
$15-25$ & very fine sand & 20 & 153 & 458 & 21 \\
$25-35$ & very fine sand & 42 & 181 & 414 & 21 \\
$35-45$ & fine sand & 35 & 344 & 716 & 22 \\
$45-55$ & very fine sand & 12 & 122 & 557 & 61 \\
$55-65$ & very fine sand & 10 & 133 & 561 & 50 \\
$65-75$ & very fine sand & 9 & 117 & 523 & 52 \\
$75-85$ & very fine sand & 12 & 113 & 570 & 67 \\
$85-95$ & very fine sand & 9 & 107 & 462 & 62 \\
\hline
\end{tabular}

TABLE A.18: Particle size and water content for site T27-A (3). Layer is specified as distance from surface. 


\section{A.19 Site description Owens Lake T27-S ${ }^{18}$}
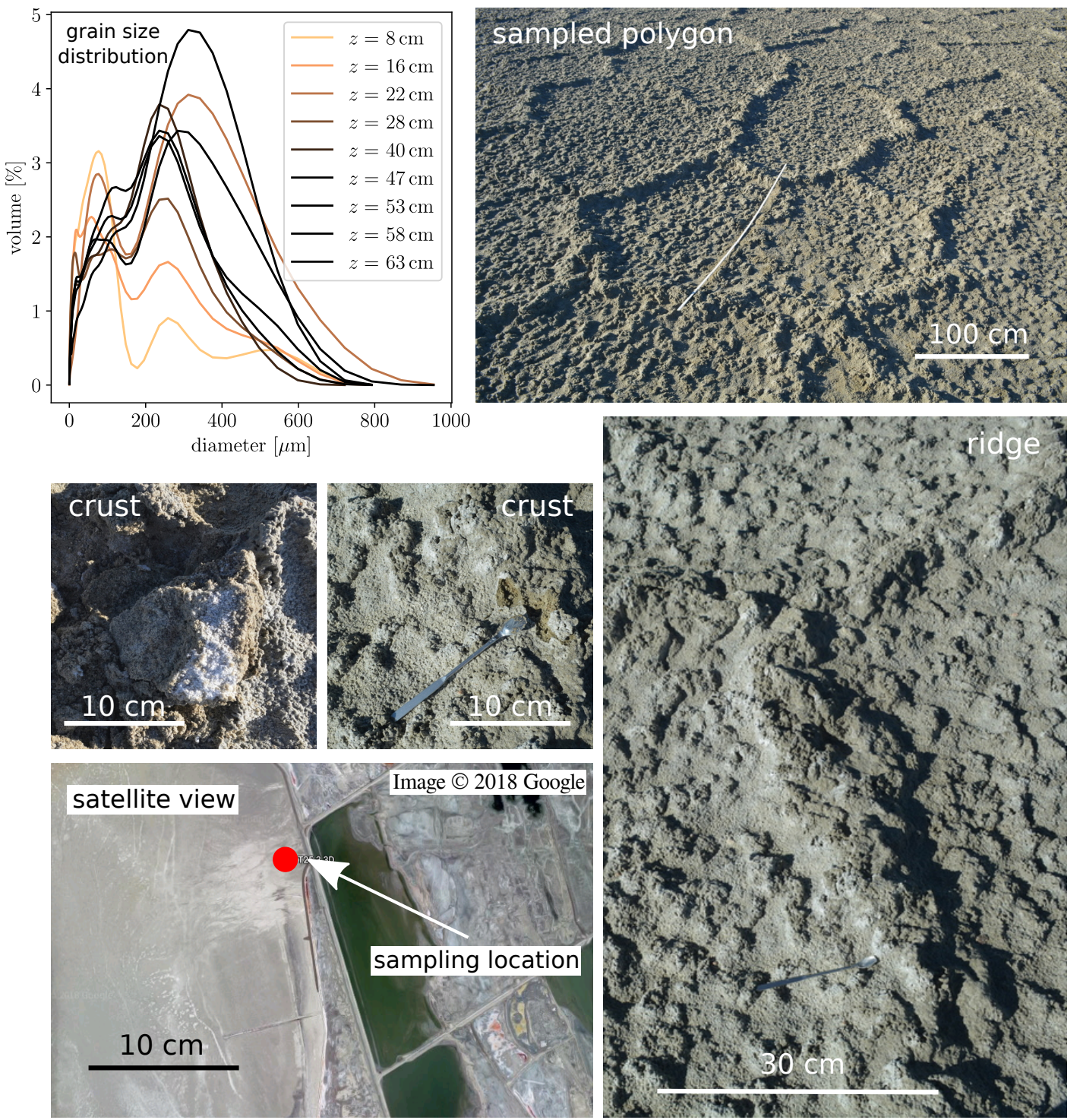

site T27-S, core 15

\begin{tabular}{c|c|c|c|c|c}
\hline layer $[\mathrm{cm}]$ & class & $d_{10}[\mu \mathrm{m}]$ & $d_{50}[\mu \mathrm{m}]$ & $d_{90}[\mu \mathrm{m}]$ & water [wt.\%] \\
\hline $5-10$ & very fine sand & 7 & 64 & 248 & 34 \\
$10-16$ & medium silt & 6 & 63 & 484 & 40 \\
$16-22$ & medium sand & 38 & 229 & 864 & 44 \\
$22-28$ & very fine sand & 6 & 71 & 548 & 29 \\
$28-47$ & fine sand & 8 & 141 & 592 & 42 \\
$28-47$ & medium sand & 12 & 163 & 784 & 60 \\
$47-57$ & medium sand & 12 & 305 & 832 & 40 \\
$47-57$ & fine sand & 11 & 163 & 606 & 206 \\
$57-63$ & fine sand & 9 & 149 & 633 & 228 \\
\hline
\end{tabular}

TABLE A.19: Particle size and water content for site T27-S. Layer is specified as distance from surface.

\footnotetext{
${ }^{18}$ Source of satellite image Google Earth, 2018-11-24
} 


\section{A.20 Site description Owens Lake T29-3 (1) ${ }^{19}$}
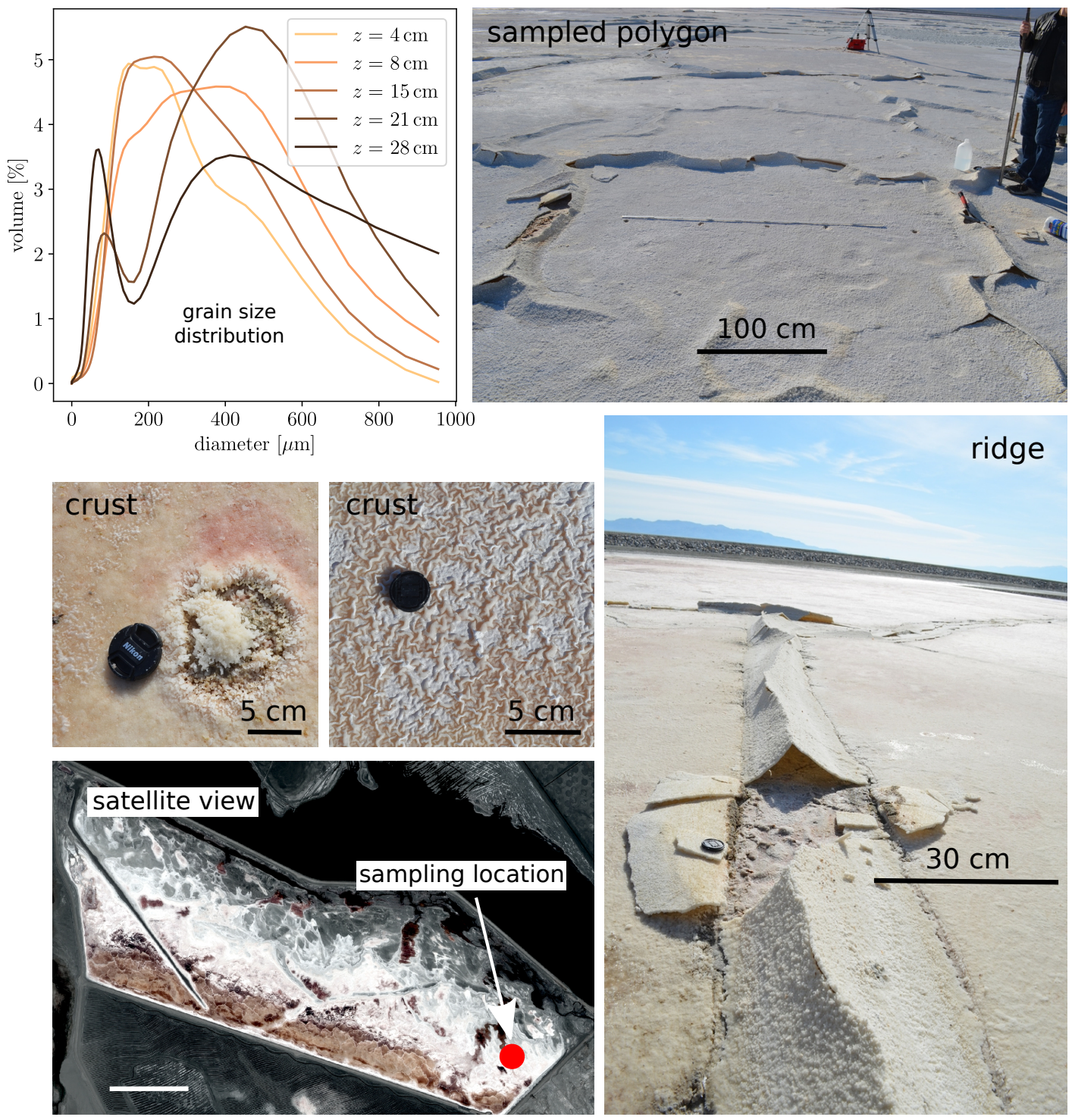

site T29-3 (1), core 3

\begin{tabular}{c|c|c|c|c|c}
\hline layer $[\mathrm{cm}]$ & class & $d_{10}[\mu \mathrm{m}]$ & $d_{50}[\mu \mathrm{m}]$ & $d_{90}[\mu \mathrm{m}]$ & water [wt.\%] \\
\hline $2-6$ & fine sand & 128 & 360 & 914 & 23 \\
$6-12$ & medium sand & 167 & 493 & 1157 & 18 \\
$12-18$ & fine sand & 181 & 429 & 1008 & 21 \\
$18-25$ & medium sand & 132 & 624 & 1311 & 19 \\
$25-31$ & medium sand & 82 & 358 & 1345 & 19 \\
\hline
\end{tabular}

TABLE A.20: Particle size and water content for site T29-3 (1). Layer is specified as distance from surface. 


\section{A.21 Site description Owens Lake T29-3 (2) ${ }^{20}$}
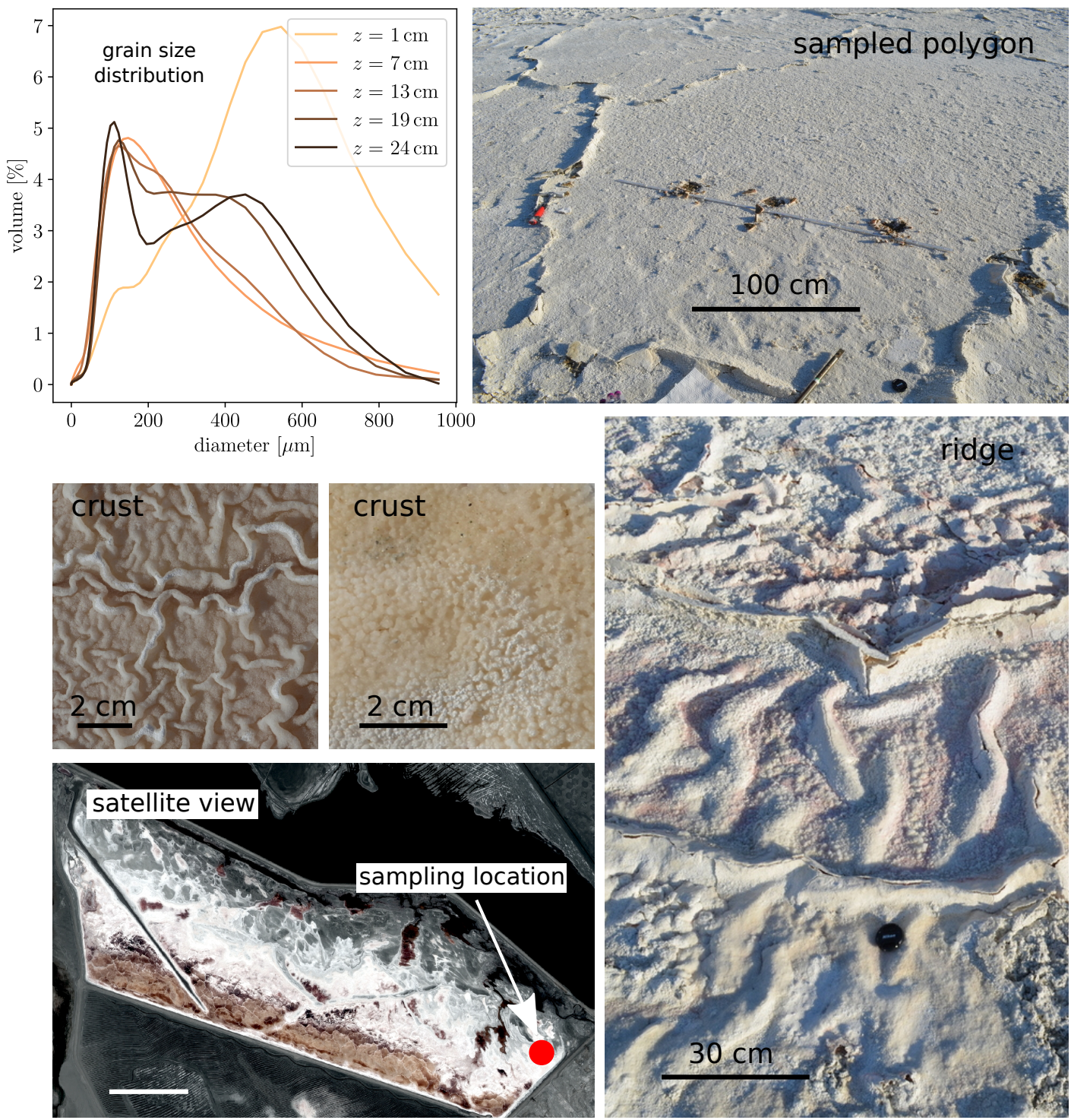

site T29-3 (2), core 3

\begin{tabular}{c|c|c|c|c|c}
\hline layer $[\mathrm{cm}]$ & class & $d_{10}[\mu \mathrm{m}]$ & $d_{50}[\mu \mathrm{m}]$ & $d_{90}[\mu \mathrm{m}]$ & water [wt.\%] \\
\hline $0-4$ & medium sand & 178 & 789 & 1446 & 32 \\
$4-10$ & fine sand & 84 & 292 & 784 & 20 \\
$10-16$ & very fine sand & 103 & 291 & 782 & 19 \\
$16-22$ & medium sand & 136 & 347 & 949 & 16 \\
$22-26$ & very fine sand & 133 & 318 & 1025 & 18 \\
\hline
\end{tabular}

TABLE A.21: Particle size and water content for site T29-3 (2). Layer is specified as distance from surface. 


\section{A.22 Site description Owens Lake T32-1-L1 (1)}

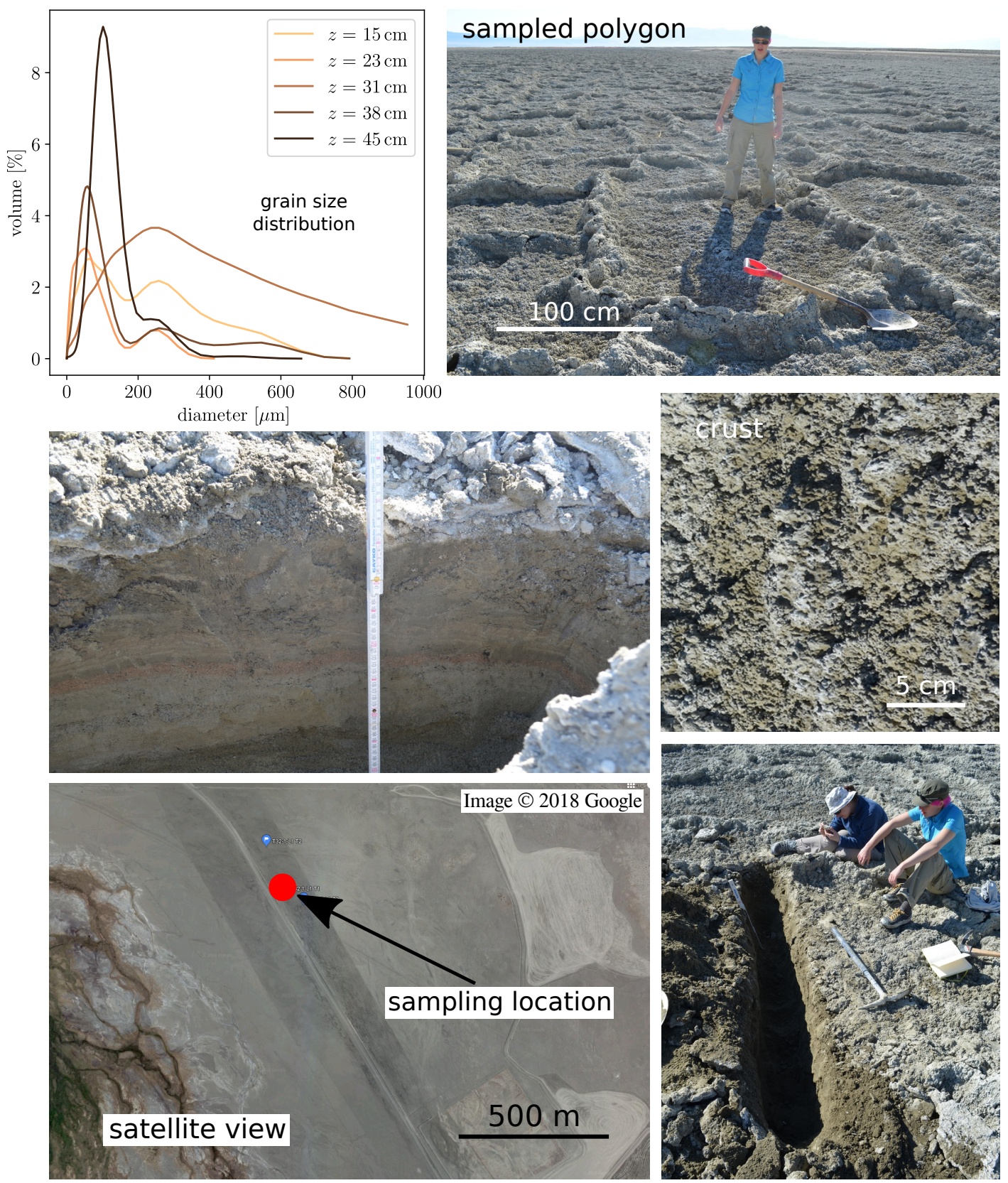

site T32-1-L1 (1), core 4

\begin{tabular}{c|c|c|c|c|c}
\hline layer $[\mathrm{cm}]$ & class & $d_{10}[\mu \mathrm{m}]$ & $d_{50}[\mu \mathrm{m}]$ & $d_{90}[\mu \mathrm{m}]$ & water [wt.\%] \\
\hline $12-18$ & very fine sand & 12 & 107 & 567 & 24 \\
$18-30$ & medium silt & 6 & 50 & 190 & 25 \\
$30-33$ & medium sand & 37 & 338 & 1090 & 16 \\
$33-39$ & coarse silt & 14 & 96 & 263 & 17 \\
$39-48$ & very fine sand & 100 & 193 & 325 & 9 \\
\hline
\end{tabular}

TABLE A.22: Particle size and water content for site T32-1-L1 (1). Layer is specified as distance from surface.

${ }^{21}$ Source of satellite image Google Earth, 2018-11-24 


\section{A.23 Site description Owens Lake T32-1-L1 (2) ${ }^{22}$}
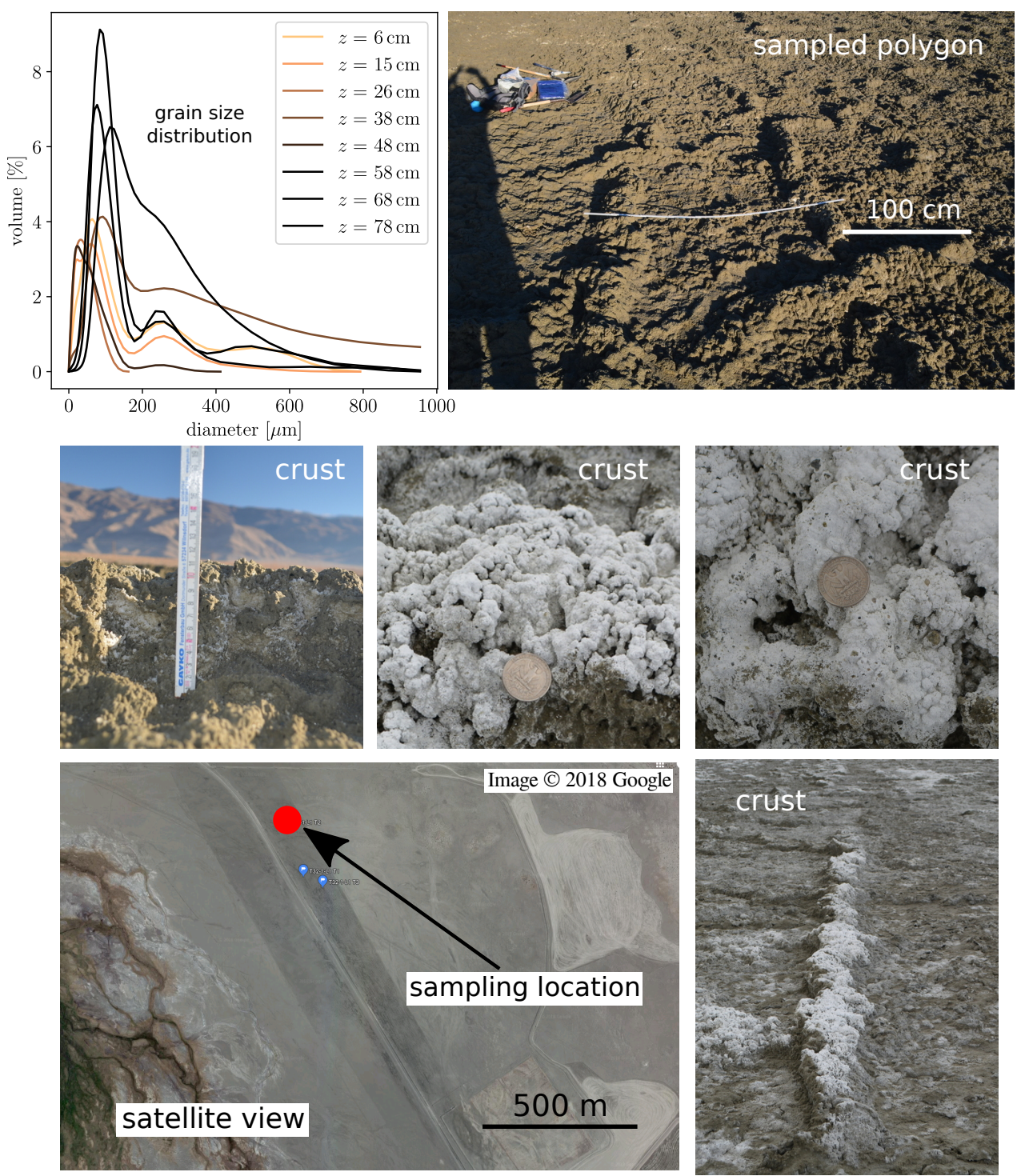

site T32-1-L1 (2), core 9

\begin{tabular}{c|c|c|c|c|c}
\hline layer [cm] & class & $d_{10}[\mu \mathrm{m}]$ & $d_{50}[\mu \mathrm{m}]$ & $d_{90}[\mu \mathrm{m}]$ & water [wt.\%] \\
\hline $1-11$ & very fine sand & 13 & 99 & 423 & 24 \\
$11-21$ & medium silt & 14 & 65 & 232 & 28 \\
$21-32$ & medium silt & 6 & 41 & 125 & 42 \\
$32-43$ & very fine sand & 28 & 195 & 844 & 13 \\
$43-53$ & medium silt & 7 & 43 & 151 & 31 \\
$53-63$ & very fine sand & 68 & 153 & 455 & 29 \\
$63-73$ & very fine sand & 102 & 177 & 355 & 31 \\
$73-83$ & very fine sand & 146 & 282 & 674 & 29 \\
\hline
\end{tabular}

TABLE A.23: Particle size and water content for site T32-1-L1 (2). Layer is specified as distance from surface.

\footnotetext{
${ }^{22}$ Source of satellite image Google Earth, 2018-11-24
} 


\section{A.24 Site description Owens Lake T32-1-L1 (3) ${ }^{23}$}
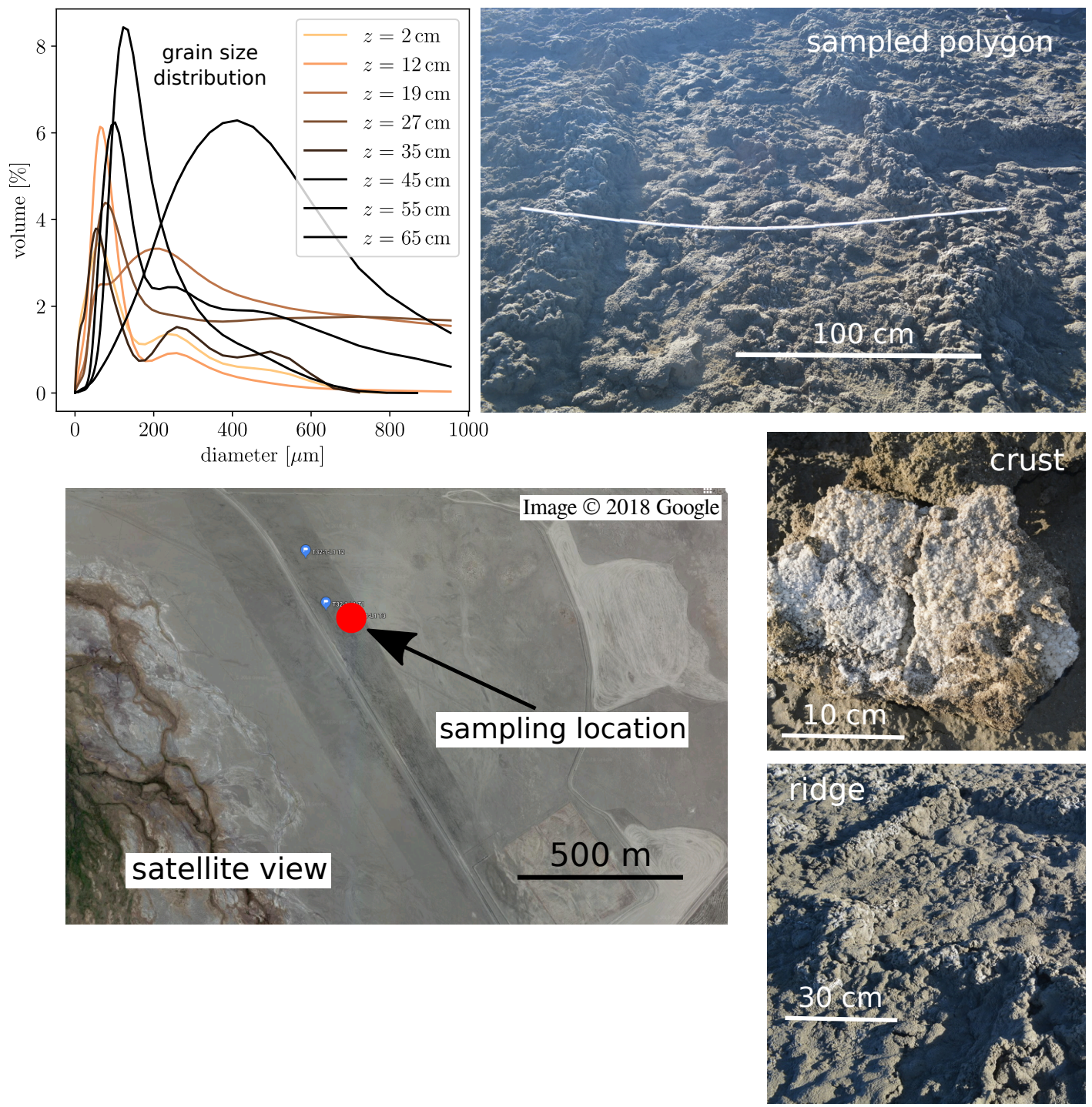

site T32-1-L1 (3), core 10

\begin{tabular}{c|c|c|c|c|c}
\hline layer $[\mathrm{cm}]$ & class & $d_{10}[\mu \mathrm{m}]$ & $d_{50}[\mu \mathrm{m}]$ & $d_{90}[\mu \mathrm{m}]$ & water [wt.\%] \\
\hline $0-18$ & very fine sand & 11 & 88 & 417 & 32 \\
$0-18$ & very fine sand & 25 & 120 & 287 & 25 \\
$18-22$ & fine sand & 59 & 300 & 1163 & 15 \\
$27-33$ & very fine sand & 67 & 204 & 1166 & 16 \\
$39-41$ & coarse silt & 9 & 89 & 498 & 19 \\
$46-65$ & fine sand & 155 & 273 & 569 & 18 \\
$46-65$ & very fine sand & 119 & 241 & 938 & 30 \\
$65-70$ & medium sand & 233 & 674 & 1322 & 30 \\
\hline
\end{tabular}

TABLE A.24: Particle size and water content for site T32-1-L1 (3). Layer is specified as distance from surface. 


\section{A.25 Site description Owens Lake T36-3 (1) ${ }^{24}$}
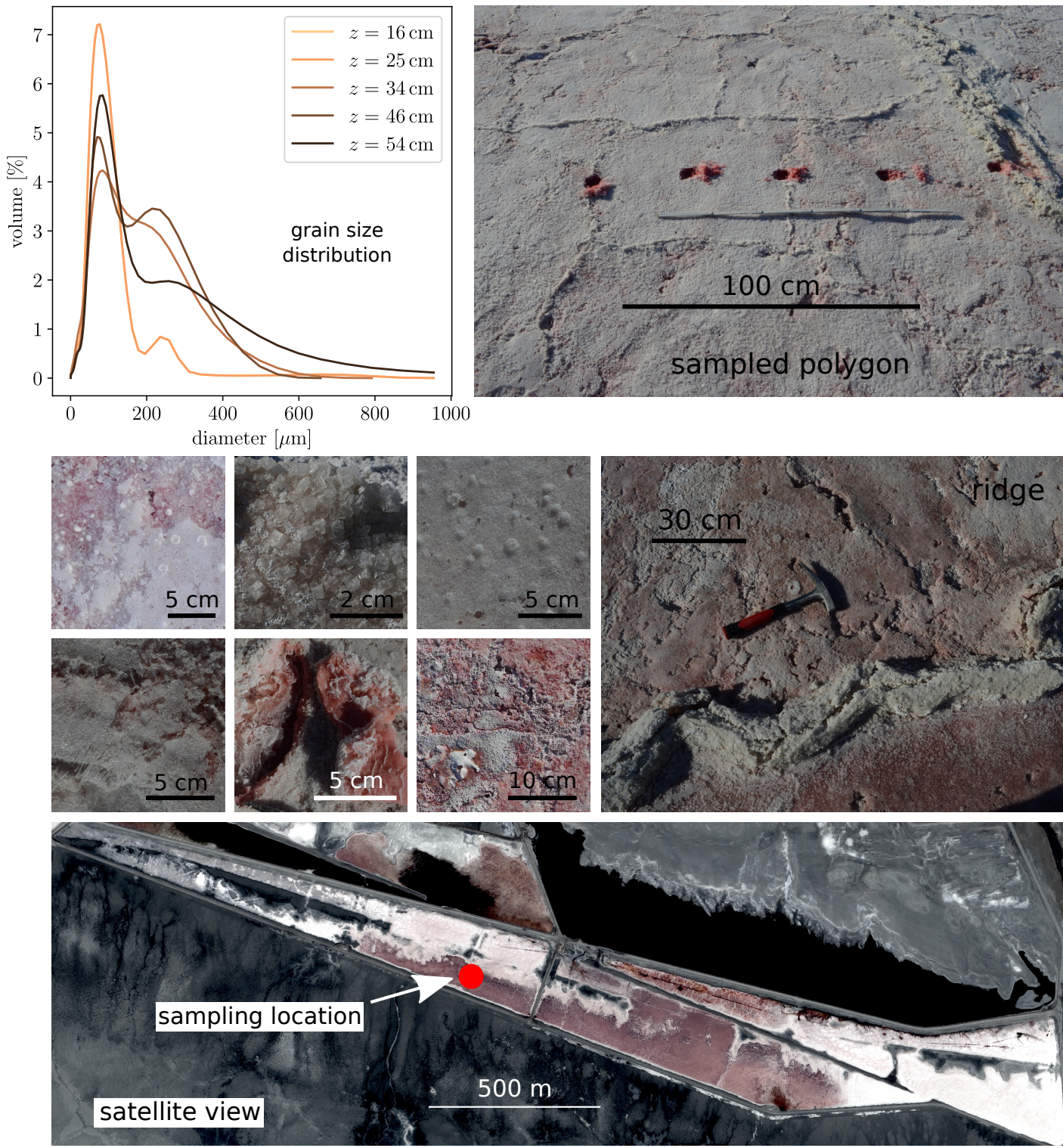

site T36-3 (1), core 3

\begin{tabular}{c|c|c|c|c|c}
\hline layer $[\mathrm{cm}]$ & class & $d_{10}[\mu \mathrm{m}]$ & $d_{50}[\mu \mathrm{m}]$ & $d_{90}[\mu \mathrm{m}]$ & water [wt.\%] \\
\hline $12-21$ & 0 & 0 & 0 & 0 & 56 \\
$21-30$ & very fine sand & 46 & 138 & 257 & 24 \\
$30-40$ & very fine sand & 35 & 176 & 521 & 35 \\
$50-58$ & very fine sand & 64 & 185 & 548 & 19 \\
$58-65$ & very fine sand & 63 & 177 & 611 & 24 \\
\hline
\end{tabular}

TABLE A.25: Particle size and water content for site T36-3 (1). Layer is specified as distance from surface.

\footnotetext{
${ }^{24}$ Satellite image courtesy Grace Holder, LADWP
} 


\section{A.26 Site description Owens Lake T36-3 (2) ${ }^{25}$}
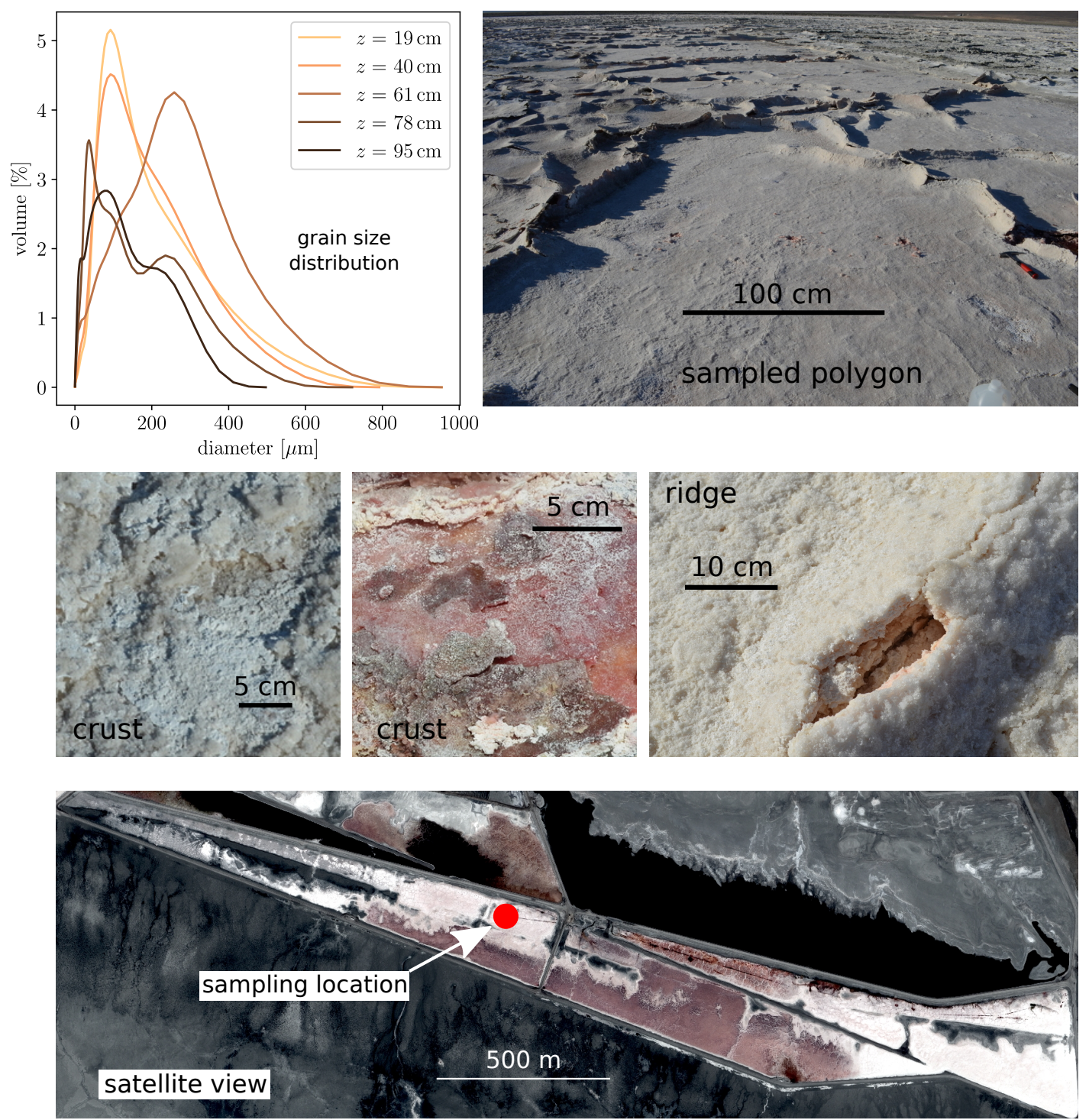

site T36-3 (2), core 3

\begin{tabular}{c|c|c|c|c|c}
\hline layer $[\mathrm{cm}]$ & class & $d_{10}[\mu \mathrm{m}]$ & $d_{50}[\mu \mathrm{m}]$ & $d_{90}[\mu \mathrm{m}]$ & water [wt.\%] \\
\hline $0-30$ & very fine sand & 63 & 195 & 555 & 21 \\
$30-51$ & very fine sand & 47 & 190 & 538 & 17 \\
$51-70$ & medium sand & 13 & 242 & 716 & 56 \\
$70-87$ & coarse silt & 15 & 90 & 451 & 84 \\
$87-103$ & very fine sand & 9 & 82 & 363 & 79 \\
\hline
\end{tabular}

TABLE A.26: Particle size and water content for site T36-3 (2). Layer is specified as distance from surface. 


\section{A.27 Site description T36-3 Owens Lake (3) ${ }^{26}$}
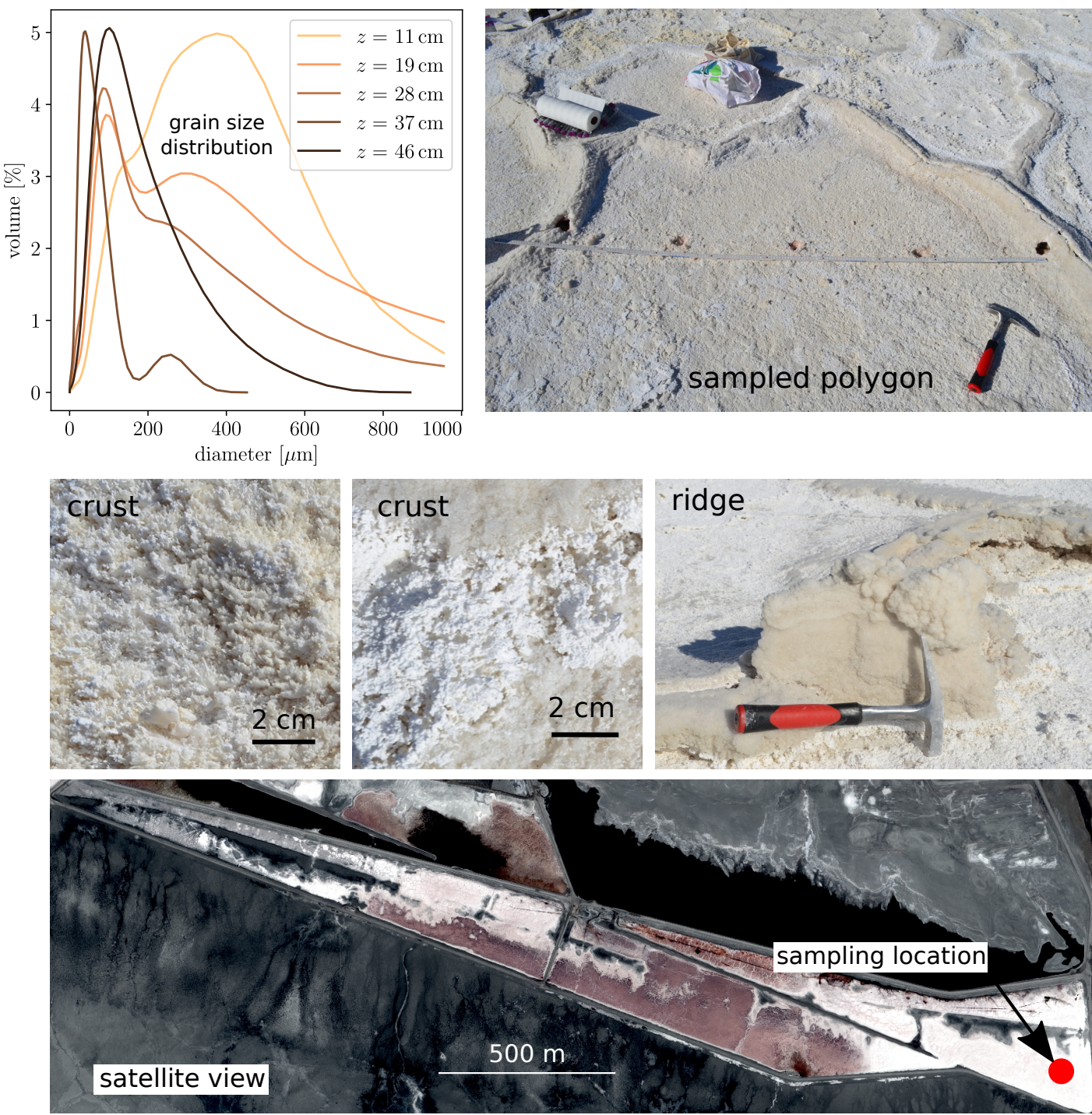

site T36-3 (3), core 3

\begin{tabular}{c|c|c|c|c|c}
\hline layer $[\mathrm{cm}]$ & class & $d_{10}[\mu \mathrm{m}]$ & $d_{50}[\mu \mathrm{m}]$ & $d_{90}[\mu \mathrm{m}]$ & water [wt.\%] \\
\hline $0-15$ & medium sand & 149 & 504 & 1126 & 18 \\
$15-24$ & very fine sand & 81 & 283 & 1059 & 19 \\
$24-33$ & very fine sand & 40 & 193 & 754 & 17 \\
$33-42$ & coarse silt & 23 & 76 & 185 & 20 \\
$42-50$ & very fine sand & 69 & 199 & 518 & 21 \\
\hline
\end{tabular}

TABLE A.27: Particle size and water content for site T36-3 (3). Layer is specified as distance from surface. 


\section{A.28 Site description Owens Lake T8-W ${ }^{27}$}
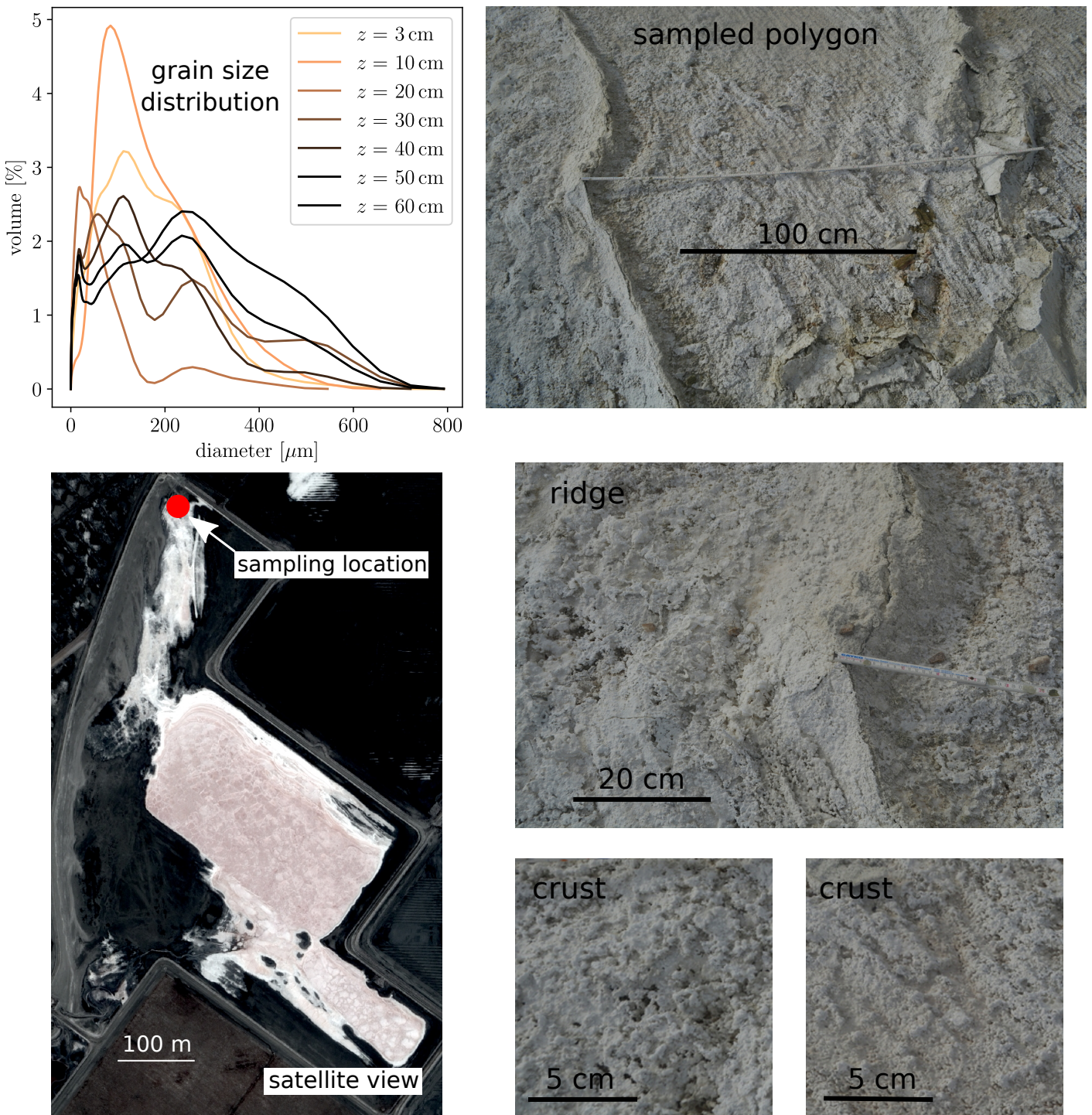

site T8-W, core 8

\begin{tabular}{c|c|c|c|c|c}
\hline layer $[\mathrm{cm}]$ & class & $d_{10}[\mu \mathrm{m}]$ & $d_{50}[\mu \mathrm{m}]$ & $d_{90}[\mu \mathrm{m}]$ & water [wt.\%] \\
\hline $0-7$ & very fine sand & 8 & 118 & 437 & 27 \\
$7-15$ & very fine sand & 32 & 171 & 463 & 24 \\
$15-25$ & clay & 3 & 30 & 140 & 30 \\
$25-35$ & clay & 4 & 62 & 453 & 33 \\
$35-45$ & very fine sand & 4 & 62 & 381 & 47 \\
$45-55$ & clay & 3 & 58 & 519 & 50 \\
$55-65$ & clay & 3 & 70 & 647 & 58 \\
\hline
\end{tabular}

TABLE A.28: Particle size and water content for site T8-W. Layer is specified as distance from surface.

\footnotetext{
${ }^{27}$ Satellite image courtesy Grace Holder, LADWP
} 


\section{Appendix B}

\section{Mineral quantification}

\section{B.1 Pore water minerals}

I used quantitative XRD (methodology described in section 3.3.3) to analyze the composition of minerals in the pore water. The results are listed in table B.2 and show that Halite is the main component of almost all brines with proportions between $38.3 \%$ to $83.1 \%$ mass.

The quantification of the scattering data showed large amorphous components. I interpreted these amorphous components as Therandite $\left(\mathrm{Na}_{2} \mathrm{SO}_{4}\right)$ which had drawn significant amounts of crystal water and transformed into Mirabilite $\left(\mathrm{Na}_{2} \mathrm{SO}_{4} \cdot 10 \mathrm{H}_{2} \mathrm{O}\right)$, which did not show a proper crystal structure anymore. This also makes sense in the context of my field observations. Especially at Owens Lake I frequently observed the expulsion of crystal water as temperatures increased during the day. Other salts present in the sampled brine also indicate a substantial presence of sulfur in the area. Before XRD analysis, samples were dried at $80^{\circ} \mathrm{C}$ and should have expelled all crystal water. Nevertheless since the quantitative XRD scans of the powder surfaces took several hours per sample, the crystal powder had enough time to draw water from the humidity in the room air. See section B.3 for a further discussion of this interpretation.

\begin{tabular}{|l|c|}
\hline Mineral & chem. composition \\
\hline Albite & $(\mathrm{NaCa}) \mathrm{Al}(\mathrm{SiAl})_{3} \mathrm{O}_{8}$ \\
Bassanite & $2(\mathrm{Ca}) 2\left(\mathrm{SO}_{4}\right) \cdot\left(\mathrm{H}_{2} \mathrm{O}\right)$ \\
Buchwaldite & $\mathrm{NaCaPO}_{4}$ \\
Burkeite & $\mathrm{Na}_{6}\left(\mathrm{CO}_{3}\right)\left(\mathrm{SO}_{4}\right)_{2}$ \\
Calcite & $\mathrm{CaCO}_{3}$ \\
Glauberite & $\mathrm{CaNa}_{2} \mathrm{SO}_{4}$ \\
Halite & $\mathrm{NaCl}$ \\
Huntite & $\mathrm{Mg}_{3} \mathrm{Ca}\left(\mathrm{CO}_{3}\right)_{4}$ \\
& $(\mathrm{NaCa})_{3}\left(\mathrm{MnFe}_{3}\right.$ \\
Janhaugite & $(\mathrm{TiZrNb})_{2} \mathrm{Si}_{4} \mathrm{O}_{15}(\mathrm{OHFO})_{3}$ \\
& $\mathrm{Na}_{3} \mathrm{SO}_{4} \mathrm{~F}$ \\
Kogarkoite & $\mathrm{Na}_{2} \mathrm{SO}_{4} \cdot 10 \mathrm{H}_{2} \mathrm{O}$ \\
Mirabilite & $\mathrm{NaHCO}_{3}$ \\
Nahcolite & $\mathrm{Na}_{2} \mathrm{CO}_{3}$ \\
Natrite & $\mathrm{KSi}_{3} \mathrm{AlO}_{8}$ \\
Orthoclase & $\mathrm{KFe}_{2} \mathrm{Al}_{2}\left(\mathrm{Al}_{2} \mathrm{Si}_{2}\right) \mathrm{O}_{1} \mathrm{O}\left(\mathrm{FOH}_{2}\right)$ \\
Siderophyllite & $\mathrm{K}_{2} \mathrm{Ca}_{2} \mathrm{H}_{2} \mathrm{O}$ \\
Syngenite & $\mathrm{Na}_{2} \mathrm{SO}_{4}$ \\
Thenardite & $\mathrm{Na}_{2} \mathrm{CO}_{3} \mathrm{H}_{2} \mathrm{O}$ \\
Thermonatrite & $\mathrm{Na}_{3}\left(\mathrm{HCO}_{3}\right)\left(\mathrm{CO}_{3}\right) 2 \mathrm{H}_{2} \mathrm{O}$ \\
Trona &
\end{tabular}

TABLE B.1: List of minerals found in saline pore water and salt crust, their chemical composition, solubility at $20^{\circ} \mathrm{C}$ and diffusion rate for single ion diffusion at $20^{\circ} \mathrm{C}$ and infinite dilution. 
Pore water minerals

\begin{tabular}{l|ll|lc|lc|lc}
\hline Site / sample & mineral 1 & {$[\%]$} & mineral 2 & {$[\%]$} & mineral 3 & {$[\%]$} & mineral 4 & {$[\%]$} \\
\hline T27-A / W1 & Halite & 56.4 & Mirab. & 30.1 & Natrite & 5.9 & Burkeite & 4.7 \\
T27-A / W2 & Halite & 44.2 & Mirab. & 30.1 & Sylvite & 8.3 & Burkeite & 8.2 \\
T29-3 / W1 & Halite & 49.6 & Mirab. & 35.6 & Burkeite & 6.2 & - & - \\
T29-3 / W2 & Halite & 52.3 & Mirab. & 35.1 & Burkeite & 5.3 & Sylvite & 2.9 \\
T32-1-L1 & Halite & 53.0 & Mirab. & 35.7 & Buchwaldite & 3.8 & Sylvite & 3.3 \\
T36-3 / W1 & Halite & 43.1 & Mirab. & 31.1 & Kogar. & 8.7 & Burkeite & 7.0 \\
T36-3 / W2 & Halite & 64.1 & Mirab. & 24.9 & Burkeite & 3.4 & Sylvite & 3.7 \\
T36-3 / W3 & Halite & 62.4 & Mirab. & 23.0 & Burkeite & 5.6 & Sylvite & 3.7 \\
T2-5 & Therm. & 42.9 & Halite & 38.3 & Natrite & 11.2 & Mirabilite & 5.8 \\
T2-4 & Halite & 49.0 & Mirab. & 20.2 & Buchwald. & 8.3 & Sylvite & 5.1 \\
Badwater / W1 & Halite & 83.1 & Glaub. & 15.3 & Syngen. & 1.5 & Mirab. & -1.4 \\
Badwater / W2 & Halite & 105.75 & Bassan. & 3.6 & - & - & Mirab. & -9.3 \\
\hline
\end{tabular}

TABLE B.2: Analysis of minerals in the pore water samples using quantitative $\mathrm{XRD}$, proportions are given in mass \%. One to three samples per area / management cell were analyzed. Only the four most common minerals are listed. Minerals with a proportion $<1 \%$ are not listed. Amorphous proportions were interpreted as Mirabilite.

Other commonly found components are Burkeite, Glauberite, Sylvite and Thermonatrite. The analysis also shows traces of phosphate salts, potassium salts, nitrates and carbonates such as Trona, which is also mined in the area. A table of the chemical composition of all relevant minerals can be found in the table B.1.

Salt species analysis for both Badwater Basin sites yields negative results for the amorphous part and, in one case, proportions of $\mathrm{NaCl}$ greater than $100 \%$ (both indicated in red). This result is not physical and points to an inability of the program AutoQuan used for the quantification to assign correct proportions to the measured diffraction peaks. Nevertheless, I did not observe any "melting" salt crusts while working at Badwater Basin and salt crystals mostly visually resembled the cubic crystalline structure of Halite. Therefore assuming a very high proportion of sodium chloride in the salt mixture from Badwater Basin seems warranted.

Analysis of XRD data with respect to reliability and a comparison with an ion quantification using ICP-OES (inductively coupled plasma optical emission spectrometry) is given in section B.3.

\section{B.2 Crust minerals}

The salt crust visibly separated into different types of crystallization patterns and salt species as shown in Fig. 3.18. I collected crust salt samples from patches of crust that looked differently to analyze the present minerals and compare to each other as well as the minerals present in the pore water. Table B.3 shows a quantification of the mineral components of the crust from a quantitative XRD analysis (as described in 3.3.3). A table of the chemical composition of all relevant minerals can be found in table B.1.

When compared to the minerals present in the pore water listed in table B.2, the different composition immediately becomes apparent: 
- For most samples, Trona is the most prevalent mineral in the crust.

- For almost all samples, Halite is only contained to a very low percentage in the crust. On the other hand, two samples are pure Halite.

- Carbonates such as Albite, Burkeite and Nahcolite are significantly more prevalent in the crust.

- Sulfur is present in various forms, such as Burkeite and Therandite.

These observations are consistent with the fact that silicates and carbonates have a significantly lower solubility than Halite and Therandite and will tend to precipitate first as the salinity of the brine reaches maximum. This region-wise separation of precipitates, so called "bull's-eyes" patterns have been described before for saline lakes [197]. Trace materials such as Manganese, Fluoride and Aluminum are present in very low quantities both in the pore water and crust (data not shown in tables).

Crust minerals

\begin{tabular}{l|lc|lc|lc|lc}
\hline Site / sample & mineral 1 & {$[\%]$} & mineral 2 & {$[\%]$} & mineral 3 & {$[\%]$} & mineral 4 & {$[\%]$} \\
\hline T2-5 / C1 & Albite & 35 & Calcite & 35 & Quartz & 15 & Orthoc. & 11 \\
T2-5 / C2 & Burkeite & 52 & Nahcolite & 23 & Trona & 15 & Halite & 6 \\
T2-5 / C3 & Trona & 46 & Nahcolite & 29 & Burkeite & 20 & Halite & 4 \\
T2-5 / C4 & Trona & 72 & Burkeite & 15 & Sideroph. & 8 & Halite & 4 \\
T2-5 / C5 & Trona & 76 & Burkeite & 15 & Huntite & 5 & Halite & 4 \\
T2-5 / C6 & Trona & 47 & Halite & 25 & Therandite & 17 & Janhaug. & 9 \\
T10-3 / C1 & Halite & 33 & Trona & 31 & Burkeite & 24 & Therand. & 12 \\
T10-3 / C2 & Halite & 100 & - & - & - & - & - & - \\
T10-3 / C3 & Halite & 100 & - & - & - & - & - & - \\
T10-3 / C4 & Trona & 40 & Therandite & 30 & Burkeite & 24 & Halite & 6 \\
\hline
\end{tabular}

TABLE B.3: Analysis of minerals from samples taken from the crust using qualitative XRD, proportions are given in \% mass. Only samples from site T2-5 and T10-3 were analyzed to compare results with the pore water minerals listed in table B.2. Only the four most common minerals for each sample are listed. Minerals with a proportion $<1 \%$ are not listed.

\section{B.3 Quantification of dissolved ions}

Among the observables entering the Rayleigh number, the diffusion coefficient and therefore the mixture of ions in the fluid remains the most elusive variable - next to the evaporation rate, where I had to base estimates entirely on measurements from literature. While the uncertainty in Ra is most certainly dominated by the uncertainty in $E$, an accurate assessment of the mineral composition is also of interest for the resident scientists and inhabitants, given the past concerns about the toxicity of the precipitated salts, and warrants additional effort.

I had concerns about the reliability of the mineral quantification obtained by quantitative XRD (described in section B.1 and section B.1), because they showed high proportions of amorphous materials which could not be quantified by XRD. 
I interpreted these amorphous sample proportions as Mirabilite which appeared amorphous due to the crystal water. To secure this interpretation I used used ICPOES to quantify the ions in the samples and compare the results to the XRD measurements (see section. 3.3.3 for a description of the measurement protocol). Results of the ICP-OES measurements for the ions $\mathrm{Na}, \mathrm{S}, \mathrm{Ca}, \mathrm{K}$ and As for several sites at Owens Lake and Badwater Basin are listed in table B.4 (subscript "I"). Next to the results from the ICP-OES I show the results from the XRD measurements converted into ion masses (subscript "X").

Values indicated in red surpassed the calibration values of the ICP-OES. Since the calibration curve saturates for very high values, prevalence of these ions will be underestimated significantly the farther away the true value is from the maximum calibration point. I note that within the limitations of the measurement apparatus, measurements yield comparable and consistent results for all sites except T2-5. I suspect that samples from this site were accidentally swapped or mis-labeled in the process. Also noticeable are the high amounts of arsenic for the Owens Lake sites, which range between 2 and $154 \mathrm{mg} / \mathrm{L}$. While this is far above the maximum levels for arsenic in potable water, it is in the range of tolerable values for arsenic in soil in Germany (as of November 2018).

\begin{tabular}{l|cc|cc|cc|cc|c} 
Site / sample & $\mathrm{Na}$ [g/L] & $\mathrm{Na}_{I}[\mathrm{~g} / \mathrm{L}]$ & $\mathrm{S}_{X}[\mathrm{~g} / \mathrm{L}]$ & $\mathrm{S}_{\mathrm{I}}[\mathrm{g} / \mathrm{L}]$ & $\mathrm{Ca}$ [ $\mathrm{mg} / \mathrm{L}]$ & $\mathrm{Ca}_{\mathrm{I}}[\mathrm{mg} / \mathrm{L}]$ & $\mathrm{K}_{X}[\mathrm{~g} / \mathrm{L}]$ & $\mathrm{K}_{\mathrm{I}}[\mathrm{g} / \mathrm{L}]$ & $\mathrm{As}_{\mathrm{I}}[\mathrm{mg} / \mathrm{L}]$ \\
\hline T27-A / W1 & 12.9 & 14.6 & 1.6 & 1.3 & 0 & 3 & 0.6 & 1.3 & 19 \\
T27-A / W2 & 22.7 & 26.8 & 4.4 & 2.0 & 0 & 2 & 4.6 & 2.9 & 37 \\
T29-3 / W1 & 8.6 & 11.1 & 1.4 & 0.9 & 0 & 7 & 0.8 & 0.7 & 11 \\
T29-3 / W2 & 173.9 & 48.8 & 27.3 & 10.1 & 0 & 27 & 16.6 & 7.6 & 154 \\
T32-1-L1 & 2.2 & 2.8 & 0.3 & 0.2 & 79 & 2 & 0.2 & 0.3 & 2 \\
T36-3 / W1 & 29.3 & 31.6 & 5.8 & 1.2 & 0 & 7 & 4.9 & 1.4 & 35 \\
T36-3 / W2 & 21.5 & 22.3 & 2.3 & 1.5 & 0 & 2 & 1.9 & 1.6 & 19 \\
T36-3 / W3 & 7.5 & 6.7 & 0.6 & 0.4 & 0 & 4 & 0.5 & 0.5 & 5 \\
T2-5 & 18.3 & 32.2 & 0.3 & 1.4 & 1328 & 5 & 0.1 & 1.2 & 16 \\
T2-4 & 18.7 & 19.3 & 2.2 & 2.2 & 0 & 3 & 1.7 & 2.2 & 45 \\
Badwater / W1 & 8.7 & 6.8 & 0.9 & 1.6 & 537 & 794 & 0.2 & 0.6 & 0 \\
Badwater / W2 & 53.2 & 34.6 & 1.1 & 1.2 & 1375 & 1254 & 0.0 & 0.2 & 0 \\
\hline
\end{tabular}

TABLE B.4: Total dissolved salts analyzed by a) quantitative XRD (subscript "X") and b) ICP-OES (subscript "I"). Values in red exceeded the calibration value of the ICP-OES and could potentially be significantly underestimated. Concentrations of Arsenic as measured with the ICP-OES are added to the table, since they are of interest as potential health hazard. 


\section{Appendix C}

\section{Owens Lake microbiology}
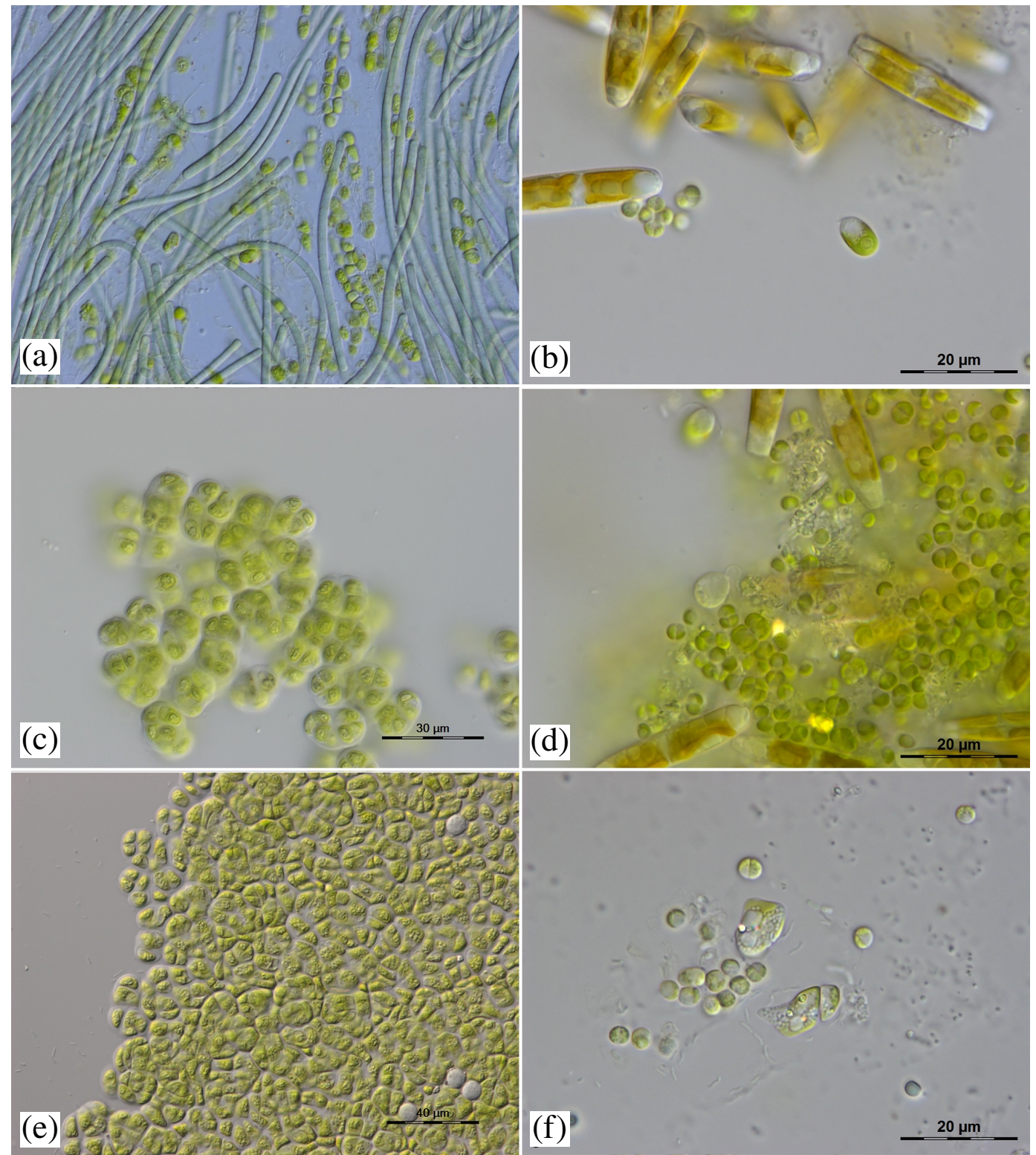

FiguRE C.1: Eucaryotic Algae and Cyanobacteria: (a) Filamentous Cyanobacteria of genus Leptolyngbya (Leptolyngbya sp.) and green algae of genus Dunaliella (Dunaliella sp.) (b) Diatoms of genus Nitzschia (Nitzschia sp.); Green Algae Dunaliella sp. and Nannochloris sp. (c) Dunaliella sp. and Nitzschia sp. (Bacillariophyta) (d) Nannochloris sp., Dunaliella sp. and Nitzschia sp. (e) Dunaliella sp. (f) Nannochloris sp. and Dangeardinella sp. Images courtesy Maike Lorenz, Göttingen Algae Collection. 

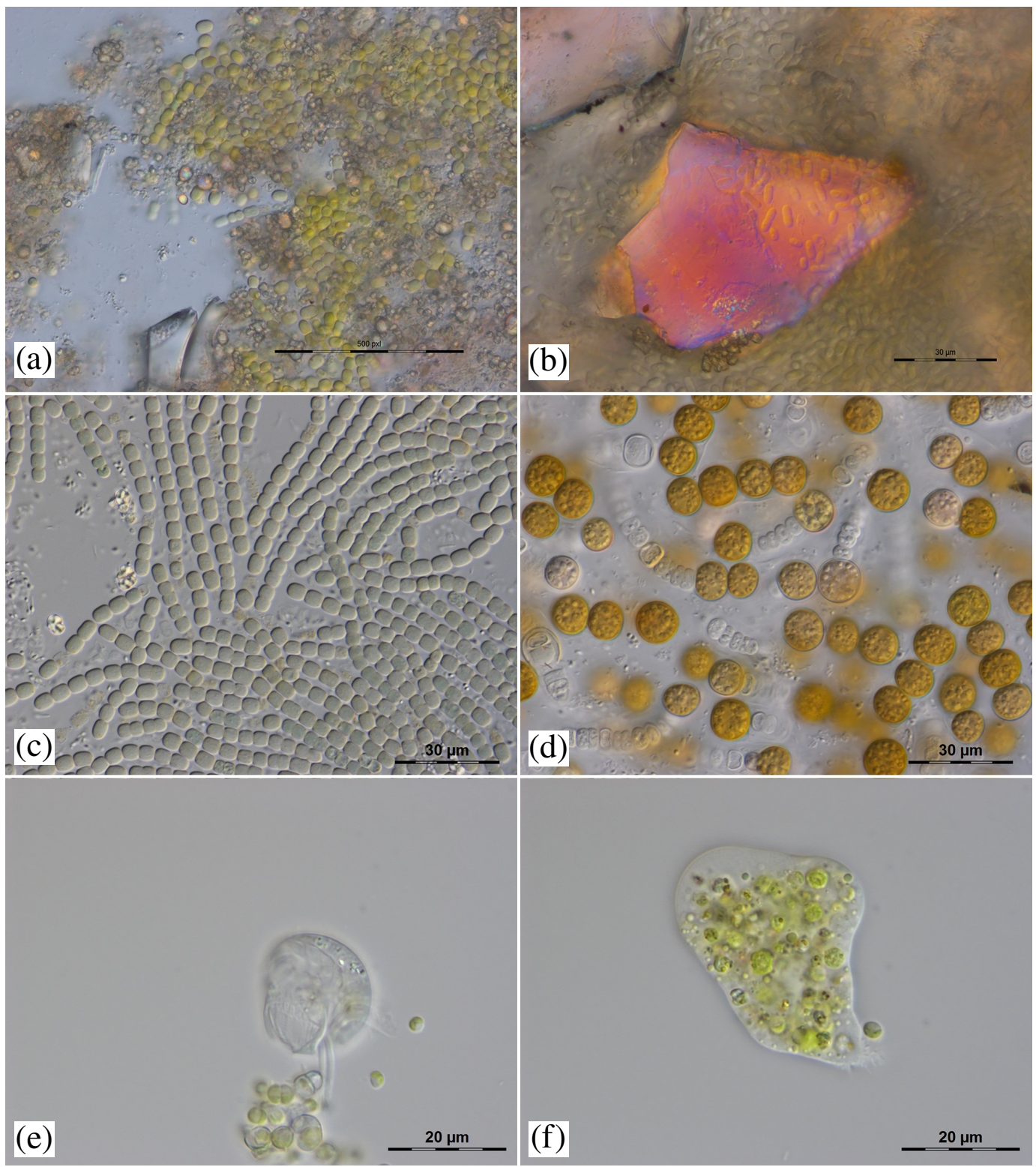

FIGURE C.2: Eucaryotic Algae, Cyanobacteria and Protistan predators: (a) Halothece californica (Cyanobacteria) (b) Synechococcus sp (Cyanobacteria) (c) Halothece Californica (d) Cysts of cyanobacterium Nostoc sp. (e) Ciliophora (formerly called Ciliata (Wimpertierchen)) feeding on algae (f) Amoeba feeding on algae. Images courtesy Maike Lorenz, Göttingen Algae Collection. 


\section{Bibliography}

[1] P. R. Briere. "Playa, playa lake, sabkha: Proposed definitions for old terms". In: Journal of Arid Environments 45.1 (2000), pp. 1-7. DOI: 10.1006/jare . 2000. 0633.

[2] Y. Yechieli and W. W. Wood. "Hydrogeologic processes in saline systems: playas, sabkhas, and saline lakes". In: Earth-Science Reviews 58.3 (2002), pp. 343365. DOI: https://doi .org/10.1016/S0012-8252(02)00067-3.

[3] K. J. Hollet et al. Geology and water resources of Owens Valley California. Tech. rep. 1991. DOI: $10.3133 /$ wsp2370b.

[4] H. P. Eugster and L. A. Hardie. "Saline Lakes". In: Lakes. Springer New York, 1978, pp. 237-293. DOI: 10.1007/978-1-4757-1152-3_8.

[5] M. R. Rosen. The importance of groundwater in playas: A review of playa classifications and the sedimentology and hydrology of playas. 1994. DOI: 10.1130/SPE289.

[6] C. J. Talbot et al. "Epitaxial salt reefs and mushrooms in the southern Dead Sea". In: Sedimentology 43.6 (1996), pp. 1025-1047. DOI: 10 . 1111 / j . 1365 3091.1996.tb01517.x.

[7] G. Wadge, D. J. Archer, and A. C. Millington. "Monitoring playa sedimentation using sequential radar images". In: Terra Nova 6.4 (1994), pp. 391-396. DOI: $10.1111 / j .1365-3121.1994$.tb00512.x.

[8] J. M. Nield et al. "The dynamism of salt crust patterns on playas". In: Geology 43.1 (2015), pp. 31-34. DOI: 10.1130/G36175.1.

[9] R. L. Reynolds et al. "Dust emission from wet and dry playas in the Mojave Desert, USA". In: Earth Surface Processes and Landforms 32.12 (2007), pp. 18111827. DOI: 10.1002/esp. 1515.

[10] R. G. Bryant and M. P. Rainey. "Investigation of flood inundation on playas within the Zone of Chotts, using a time-series of AVHRR". In: Remote Sensing of Environment 82.2-3 (2002), pp. 360-375. DOI: 10 . 1016 / s0034-4257 (02) 00053-6.

[11] G. R. North, R. F. Cahalan, and J. A. Coakley. “Energy balance climate models". In: Reviews of Geophysics 19.1 (1981), p. 91. DOI: 10 .1029/rg019i001p00091.

[12] A. J. Pitman. "The evolution of, and revolution in, land surface schemes designed for climate models". In: International Journal of Climatology 23.5 (2003), pp. 479-510. DOI: 10.1002/joc. 893. 
[13] T. E. Gill. "Eolian sediments generated by anthropogenic disturbance of playas: human impacts on the geomorphic system and geomorphic impacts on the human system". In: Geomorphology 17.1 (1996). Response of Aeolian Processes to Global Change, pp. 207-228. DOI: https : / / doi . org / 10 . 1016 / 0169 555X (95) 00104-D.

[14] R. Washington et al. "Dust-Storm Source Areas Determined by the Total Ozone Monitoring Spectrometer and Surface Observations". In: Annals of the Association of American Geographers 93.2 (2003), pp. 297-313. DOI: 10 .1111/14678306.9302003.

[15] J. M. Prospero. "Environmental characterization of global sources of atmospheric soil dust identified with the NIMBUS 7 Total Ozone Mapping Spectrometer (TOMS) absorbing aerosol product". In: Reviews of Geophysics 40.1 (2002). DOI: 10.1029/2000rg000095.

[16] M. R. Raupach, D. A. Gillette, and J. F. Leys. "The effect of roughness elements on wind erosion threshold". In: Journal of Geophysical Research: Atmospheres 98.D2 (1993), pp. 3023-3029. DOI: 10.1029/92jd01922.

[17] B. Marticorena and G. Bergametti. "Modeling the atmospheric dust cycle: 1. Design of a soil-derived dust emission scheme". In: Journal of Geophysical Research 100.D8 (1995), pp. 16415-16430. DOI: 10.1029/95jd00690.

[18] K. A. Koehler et al. "Potential impact of Owens (dry) Lake dust on warm and cold cloud formation". In: Journal of Geophysical Research 112.D12 (2007). DOI: 10.1029/2007jd008413.

[19] A. Seaton et al. "Particulate air pollution and acute health effects". In: The Lancet 345.8943 (1995), pp. 176-178. DOI: 10.1016/s0140-6736 (95) 90173-6.

[20] A. Robock and J. Mao. "The Volcanic Signal in Surface Temperature Observations". In: Journal of Climate 8.5 (1995), pp. 1086-1103. DOI: 10.1175/1520$0442(1995) 008<1086$ : tvsist $>2.0 . c 0 ; 2$.

[21] A. Lacis, J. Hansen, and M. Sato. "Climate forcing by stratospheric aerosols". In: Geophysical Research Letters 19.15 (1992), pp. 1607-1610. DOI: 10 . 1029 / 92g101620.

[22] J. M. Prospero. "Long-range transport of mineral dust in the global atmosphere: Impact of African dust on the environment of the southeastern United States". In: Proceedings of the National Academy of Sciences 96.7 (1999), pp. 33963403. DOI: 10.1073/pnas.96.7.3396.

[23] I. Y. Fung et al. "Iron supply and demand in the upper ocean". In: Global Biogeochemical Cycles 14.1 (2000), pp. 281-295. DOI: 10.1029/1999gb900059.

[24] V. A. Pretti and B. W. Stewart. "Solute sources and chemical weathering in the Owens Lake watershed, eastern California". In: Water Resources Research 38.8 (2002). DOI: $10.1029 / 2001$ WR000370. 
[25] S. Zhong et al. "Climatology of High Wind Events in the Owens Valley, California". In: Monthly Weather Review 136.9 (2008), pp. 3536-3552. DOI: 10 . 1175/2008MWR2348.1.

[26] J. Ryu et al. "Characterization of Redox Processes in Shallow Groundwater of Owens Dry Lake, California". In: Environmental Science \& Technology 38.22 (2004), pp. 5950-5957. DOI: 10.1021/es0306112.

[27] T. E. Gill et al. "Elemental geochemistry of wind-erodible playa sediments, Owens Lake, California". In: Nuclear Instruments and Methods in Physics 189.1 (2002), pp. 209-213. DOI: https : //doi .org/10.1016/S0168-583X(01) 010448.

[28] J. Ryu et al. "Arsenic distribution, speciation and solubility in shallow groundwater of Owens Dry Lake, California". In: Geochimica et Cosmochimica Acta 66.17 (2002), pp. 2981-2994. DOI: https : / / doi . org / 10 . 1016 / S0016 7037 (02)00897-9.

[29] J. S. Reid et al. "Local meteorological, transport, and source aerosol characteristics of late autumn Owens Lake (dry) dust storms". In: Atmospheric Environment 28.9 (1994), pp. 1699-1706. DOI: https : / / doi .org/10 . 1016/13522310 (94) 90315-8.

[30] M. C. Reheis. “Dust deposition downwind of Owens (dry) Lake, 1991-1994: Preliminary findings". In: Journal of Geophysical Research: Atmospheres $102 . D 22$ (1997), pp. 25999-26008. DOI: 10.1029/97JD01967.

[31] T. E. Gill. “Dust Generation Resulting from Desiccation of Playa Systems: Studies on Mono and Owens Lakes, California". PhD thesis. University of California, Davis., 1995. URL: http://adsabs . harvard . edu/abs/1995PhDT . .....114G.

[32] T. A. Cahill et al. "Saltating particles, playa crusts and dust aerosols at Owens (dry) Lake, California". In: Earth Surface Processes and Landforms 21.7 (1996), pp. 621-639. DOI: 10 . 1002 / (sici ) 1096 - 9837 (199607) $21: 7<621:$ : aid esp661>3.0.co;2-e.

[33] M. David. “The Owens Lake Project”. In: Niemand Reports 59.1 (2005), pp. 1719.

[34] D. P. Groeneveld and D. D. Barz. "Remote Monitoring of Surfaces Wetted for Dust Control on the Dry Owens Lakebed, California". In: Open Journal of Modern Hydrology 03.04 (2013), pp. 241-252. DOI: 10 .4236/ojmh. 2013. 34028.

[35] L. Nicholas and B. Andy. "Influence of vegetation cover on sand transport by wind: field studies at Owens Lake, California". In: Earth Surface Processes and Landforms 23.1 (1997), pp. 69-82. DOI: 10 . 1002 / (SICI) 1096-9837 (199801) 23:1<69: :AID-ESP823>3.0.CO;2-G.

[36] O. L. M. P. Committee. Owens Lake Master Project. 2013. URL: https : //www . ladwp.com/ladwp/faces/ladwp/aboutus/a-water/a-w-losangelesaqueduct. 
[37] D. Groeneveld, J. Huntington, and D. Barz. "Floating brine crusts, reduction of evaporation and possible replacement of fresh water to control dust from Owens Lake bed, California". In: Journal of Hydrology 392.3 (2010), pp. 211218. DOI: https://doi.org/10.1016/j.jhydrol.2010.08.010.

[38] R. Lorand. Aesthetic Order: A Philosophy of Order, Beauty and Art (Routledge Studies in Twentieth-Century Philosophy). Routledge, 2000.

[39] K. Kennedy, R. Bergman, and R. Johnson. Star Wars: the last Jedi. Walt Disney Pictures, Lucasfilm. 2017.

[40] M. A. Borowitzka and C. J. Siva. "The taxonomy of the genus Dunaliella (Chlorophyta, Dunaliellales) with emphasis on the marine and halophilic species". In: Journal of Applied Phycology 19.5 (2007), pp. 567-590. DOI: 10 . 1007/s10811-007-9171-x.

[41] R. A. Wooding, S. W. Tyler, and I. White. "Convection in groundwater below an evaporating Salt Lake: 1. Onset of instability". In: Water Resources Research 33.6 (1997), pp. 1199-1217. DOI: 10.1029/96WR03533.

[42] Public weather records for Lone Pine, CA. https://weatherspark.com/y/1764/ Average - Weather - in - Lone - Pine - California - United - States - Year Round. Accessed: 2018-11-30.

[43] Public weather records for Furnace Creek, CA. https: //www . worldweatheronline. com / furnace - creek - weather - history / california / us . aspx. Accessed: 2018-11-30.

[44] S. Tyler et al. "Estimation of groundwater evaporation and salt flux from Owens Lake, California, USA". In: Journal of Hydrology 200.1 (1997), pp. 110135. DOI: https://doi .org/10.1016/S0022-1694(97)00007-3.

[45] C. R. Handford. Estimated Ground-Water Discharge by Evapotranspiration from Death Valley, California, 1997-2001. Tech. rep. 2003. DOI: 10.3133/wri034254.

[46] S. Getzin et al. "Discovery of fairy circles in Australia supports self-organization theory". In: Proceedings of the National Academy of Sciences 113.13 (2016), pp. 35513556. DOI: $10.1073 /$ pnas. 1522130113.

[47] B. Werner. "Modeling Landforms as Self-Organized, Hierarchical Dynamical Systems". In: Prediction in Geomorphology. American Geophysical Union, 2013, pp. 133-150. DOI: $10.1029 / 135$ gm10.

[48] L. Goehring. "Evolving fracture patterns: columnar joints, mud cracks and polygonal terrain". In: Philosophical Transactions of the Royal Society A 371.2004 (2013), pp. 20120353-20120353. DOI: 10.1098/rsta.2012. 0353.

[49] P. Pina et al. "Analog Studies of Ice-wedge Polygons in Svalbard: 2011 Field Campaign, Topology and Geometry". In: Lunar and Planetary Science Conference. Vol. 43. Lunar and Planetary Inst. Technical Report. 2012, p. 2353. URL: http://adsabs . harvard. edu/abs/2012LPI . . . 43.2353P. 
[50] K. Thomas et al. "Formation of Kinneyia via shear-induced instabilities in microbial mats". In: Philosophical Transactions of the Royal Society A 371.2004 (2013), pp. 20120362-20120362. DOI: 10.1098/rsta.2012.0362.

[51] F. Engelund. "Instability of erodible beds". In: Journal of Fluid Mechanics 42.02 (1970), pp. 225-244. DOI: 10.1017/s0022112070001210.

[52] N. Juergens. "The Biological Underpinnings of Namib Desert Fairy Circles". In: Science 339.6127 (2013), pp. 1618-1621. DOI: 10.1126/science. 1222999.

[53] L. F. Dellwig. "Significant Features of Deposition in the Hutchinson Salt, Kansas, and Their Interpretation". In: Geological Society of America Special Papers. Geological Society of America, 1968, pp. 421-428. DOI: 10.1130/spe88p421.

[54] R. M. Tucker. "Giant polygons in the Triassic salt of Cheshire, England; a thermal contraction model for their origin". In: Journal of Sedimentary Research 51.3 (1981), pp. 779-786. DOI: 10.1306/212F7DA6-2B24-11D7-8648000102C1865D.

[55] F. W. Christiansen. "Polygonal Fracture and Fold Systems in the Salt Crust, Great Salt Lake Desert, Utah". In: Science 139.3555 (1963), pp. 607-609. DOI: 10.1126/science.139.3555.607.

[56] S. Lokier. "Development and evolution of subaerial halite crust morphologies in a coastal sabkha setting". In: Journal of Arid Environments 79 (2012), pp. 32-47. DOI: https ://doi .org/10.1016/j. jaridenv. 2011.11.031.

[57] B. Li et al. "Mechanics of morphological instabilities and surface wrinkling in soft materials: a review". In: Soft Matter 8.21 (2012), p. 5728. DOI: 10.1039/ c2sm00011c.

[58] R. A. Wooding. "Rayleigh instability of a thermal boundary layer in flow through a porous medium". In: Journal of Fluid Mechanics 9.02 (1960), p. 183. DOI: $10.1017 / \mathrm{s} 0022112060001031$.

[59] G. M. Homsy and A. E. Sherwood. "Convective instabilities in porous media with through flow". In: AIChE Journal 22.1 (1976), pp. 168-174. DOI: 10.1002/ aic. 690220121.

[60] R. A. Wooding et al. "Convection in groundwater below an evaporating Salt Lake: 2. Evolution of fingers or plumes". In: Water Resources Research 33.6 (1997), pp. 1219-1228. DOI: 10.1029/96WR03534.

[61] A. L. Hales. "Convection Currents in Geysers." In: Geophysical Journal International 4.s1 (1937), pp. 122-131. DOI: 10.1111/j.1365-246x.1937. tb00414.x.

[62] C. W. Horton and F. T. Rogers. "Convection Currents in a Porous Medium". In: Journal of Applied Physics 16.6 (1945), pp. 367-370. DOI: 10.1063/1.1707601.

[63] E. R. Lapwood. "Convection of a fluid in a porous medium". In: Mathematical Proceedings of the Cambridge Philosophical Society 44.4 (1948), pp. 508-521. DOI: 10.1017/S030500410002452X. 
[64] R. A. Wooding. "Steady state free thermal convection of liquid in a saturated permeable medium". In: Journal of Fluid Mechanics 2.3 (1957), pp. 273-285. DOI: $10.1017 /$ S0022112057000129.

[65] J. W. Elder. "Steady free convection in a porous medium heated from below". In: Journal of Fluid Mechanics 27.01 (1967), p. 29. DOI: 10.1017/s0022112067000023.

[66] R. A. Wooding. "Growth of fingers at an unstable diffusing interface in a porous medium or Hele-Shaw cell". In: Journal of Fluid Mechanics 39.3 (1969), pp. 477-495. DOI: 10.1017/S002211206900228X.

[67] H. J. G. Diersch and O. Kolditz. "Variable-density flow and transport in porous media: approaches and challenges". In: Advances in Water Resources 25.8 (2002), pp. 899-944. DOI: https://doi .org/10.1016/S0309-1708(02)00063-5.

[68] C. T. Simmons. "Variable density groundwater flow: From current challenges to future possibilities". In: Hydrogeology Journal 13.1 (2005), pp. 116-119. DOI: $10.1007 / \mathrm{s} 10040-004-0408-3$.

[69] J. P. Heller. "Onset of Instability Patterns between Miscible Fluids in Porous Media". In: Journal of Applied Physics 37.4 (1966), pp. 1566-1579. DOI: 10 . 1063/ 1.1708569 .

[70] W. R. Schowalter. "Stability criteria for miscible displacement of fluids from a porous medium". In: AIChE Journal 11.1 (1965), pp. 99-105. DOI: 10 . 1002/ aic. 690110122 .

[71] P. C. Wankat and W. R. Schowalter. "Stability of Combined Heat and Mass Transfer in a Porous Medium". In: The Physics of Fluids 13.9 (1970), pp. 24182420. DOI: $10.1063 / 1.1693253$.

[72] P. G. Saffman. "Viscous fingering in Hele-Shaw cells". In: Journal of Fluid Mechanics 173.-1 (1986), pp. 73-94. DOI: 10.1017/s0022112086001088.

[73] A. C. Slim. "Solutal-convection regimes in a two-dimensional porous medium". In: Journal of Fluid Mechanics 741 (2014), pp. 461-491. DOI: 10.1017/jfm. 2013. 673.

[74] J. W. Elder. "The unstable thermal interface". In: Journal of Fluid Mechanics 32.1 (1968), pp. 69-96. DOI: 10.1017/S0022112068000595.

[75] T. J. Kneafsey and K. Pruess. "Laboratory Flow Experiments for Visualizing Carbon Dioxide-Induced, Density-Driven Brine Convection". In: Transport in Porous Media 82.1 (2010), pp. 123-139. DOI: 10.1007/s11242-009-9482-2.

[76] M. Soroush et al. "Affecting Parameters in Density Driven Convection Mixing in CO2 Storage in Brine". In: SPE Europec/EAGE Annual Conference. Society of Petroleum Engineers, 2012. DOI: 10.2118/154901-ms.

[77] C. W. MacMinn et al. "Spreading and convective dissolution of carbon dioxide in vertically confined, horizontal aquifers". In: Water Resources Research 48.11 (2012). DOI: $10.1029 / 2012$ WR012286. 
[78] A. C. Slim et al. "Dissolution-driven convection in a Hele-Shaw cell". In: Physics of Fluids 25.2 (2013), p. 024101. DOI: 10.1063/1 . 4790511.

[79] C. W. MacMinn and R. Juanes. "Buoyant currents arrested by convective dissolution". In: Geophysical Research Letters 40.10 (2013), pp. 2017-2022. DOI: 10.1002/grl.50473.

[80] P. A. Tsai, K. Riesing, and H. A. Stone. "Density-driven convection enhanced by an inclined boundary: Implications for geological CO2storage". In: Physical Review E 87.1 (2013). DOI: 10.1103/physreve.87.011003.

[81] R. Outeda et al. "Experimental study of linear and nonlinear regimes of densitydriven instabilities induced by $\mathrm{CO}_{2}$ dissolution in water". In: Chaos 24.1 (2014), p. 013135. DOI: $10.1063 / 1.4868040$.

[82] T. F. Faisal et al. "Quantitative and qualitative study of density driven $\mathrm{CO}_{2}$ mass transfer in a vertical Hele-Shaw cell". In: International Journal of Heat and Mass Transfer 81 (2015), pp. 901-914. DOI: https ://doi .org/10.1016/j . ijheatmasstransfer.2014.11.017.

[83] A. Vreme et al. "Gravitational instability due to the dissolution of carbon dioxide in a Hele-Shaw cell". In: Physical Review Fluids 1.6 (2016). DOI: 10 . 1103/physrevfluids.1.064301.

[84] J. Ching, P. Chen, and P. A. Tsai. “Convective mixing in homogeneous porous media flow". In: Physical Review Fluids 2.1 (2017). DOI: 10 . 1103/physrevfluids . 2.014102 .

[85] C. Thomas, S. Dehaeck, and A. D. Wit. "Convective dissolution of $\mathrm{CO}_{2}$ in water and salt solutions". In: International Journal of Greenhouse Gas Control 72 (2018), pp. 105-116. DOI: https://doi.org/10.1016/j.ijggc.2018.01.019.

[86] F. M. Sutton. "Onset of Convection in a Porous Channel with Net Through Flow". In: The Physics of Fluids 13.8 (1970), pp. 1931-1934. DOI: 10 . 1063/1. 1693188.

[87] S. M. Hassanizadeh and T. Leijnse. "On the modeling of brine transport in porous media". In: Water Resources Research 24.3 (1988), pp. 321-330. DOI: 10 . 1029/WR024i003p00321.

[88] A. Kemna et al. "Imaging and characterisation of subsurface solute transport using electrical resistivity tomography (ERT) and equivalent transport models". In: Journal of Hydrology 267.3 (2002), pp. 125-146. DOI: https : / / doi . org/10.1016/S0022-1694(02)00145-2.

[89] R. A. Wooding. "Instability of a viscous liquid of variable density in a vertical Hele-Shaw cell". In: Journal of Fluid Mechanics 7.04 (1960), p. 501. DOI: 10 . $1017 / \mathrm{s} 0022112060000256$. 
[90] P. Saffman and F. Taylor. "The penetration of a fluid into a porous medium or Hele-Shaw cell containing a more viscous liquid". In: Proceedings of the Royal Society of London A 245.1242 (1958), pp. 312-329. DOI: 10 . 1098/rspa . 1958. 0085.

[91] J. D. Chen. "Radial viscous fingering patterns in Hele-Shaw cells". In: Experiments in Fluids 5.6 (1987), pp. 363-371. DOI: 10.1007/BF00264399.

[92] J. Ennis-King, I. Preston, and L. Paterson. "Onset of convection in anisotropic porous media subject to a rapid change in boundary conditions". In: Physics of Fluids 17.8 (2005). DOI: 10.1063/1.2033911.

[93] X. Xu, S. Chen, and D. Zhang. "Convective stability analysis of the longterm storage of carbon dioxide in deep saline aquifers". In: Advances in Water Resources 29.3 (2006), pp. 397-407. DOI: https : / / doi . org / 10 . 1016 / j . advwatres.2005.05.008.

[94] S. Rapaka et al. "Onset of convection over a transient base-state in anisotropic and layered porous media". In: Journal of Fluid Mechanics 641 (2009), pp. 227244. DOI: $10.1017 /$ S0022112009991479.

[95] J. Otero et al. "High-Rayleigh-number convection in a fluid-saturated porous layer". In: Journal of Fluid Mechanics 500 (2004), pp. 263-281. DOI: 10 . 1017 / S0022112003007298.

[96] M. Boufadel, M. Suidan, and A. Venosa. "Numerical modeling of water flow below dry salt lakes: effect of capillarity and viscosity". In: Journal of Hydrology 221.1 (1999), pp. 55-74. DOI: https : / / doi . org / 10 . 1016/ S0022 1694 (99) 00077-3.

[97] E. Shahraeeni, P. Lehmann, and D. Or. "Coupling of evaporative fluxes from drying porous surfaces with air boundary layer: Characteristics of evaporation from discrete pores". In: Water Resources Research 48.9 (2012). DOI: 10 . $1029 / 2012 \mathrm{wr} 011857$.

[98] E. Hamann et al. "Numerical investigation of coupled density-driven flow and hydrogeochemical processes below playas". In: Water Resources Research 51.11 (2015), pp. 9338-9352. DOI: 10.1002/2015WR017833.

[99] A. E. Charola. "Salts in the Deterioration of Porous Materials: An Overview". In: Journal of the American Institute for Conservation 39.3 (2000), pp. 327-343. DOI: $10.1179 / 019713600806113176$.

[100] K. U. Mayer, E. O. Frind, and D. W. Blowes. "Multicomponent reactive transport modeling in variably saturated porous media using a generalized formulation for kinetically controlled reactions". In: Water Resources Research 38.9 (2002), pp. 13-1-13-21. DOI: 10.1029/2001wr000862.

[101] J. L. Beck. "Convection in a Box of Porous Material Saturated with Fluid". In: The Physics of Fluids 15.8 (1972), pp. 1377-1383. DOI: 10.1063/1.1694096. 
[102] X. Fu, L. Cueto-Felgueroso, and R. Juanes. "Pattern formation and coarsening dynamics in three-dimensional convective mixing in porous media". In: Philosophical Transactions of the Royal Society of London A 371.2004 (2013). DOI: 10.1098/rsta.2012.0355.

[103] D. R. Hewitt, J. A. Neufeld, and J. R. Lister. "High Rayleigh number convection in a three-dimensional porous medium". In: Journal of Fluid Mechanics 748 (2014), pp. 879-895. DOI: 10.1017/jfm.2014. 216.

[104] M. Prat. "Recent advances in pore-scale models for drying of porous media". In: Chemical Engineering Journal 86.1-2 (2002), pp. 153-164. DOI: 10 . 1016 / s1385-8947 (01) 00283-2.

[105] S. Whitaker. "Flow in porous media I: A theoretical derivation of Darcy's law". In: Transport in Porous Media 1.1 (1986), pp. 3-25. DOI: 10.1007/BF01036523.

[106] G. Łukaszewicz and P. Kalita. Navier-Stokes Equations. Springer International Publishing, 2016. DOI: 10.1007/978-3-319-27760-8.

[107] "An analytical derivation of the Darcy equation". In: Eos, Transactions American Geophysical Union 37.2 (1956), pp. 185-188. DOI: 10 .1029/TR037i002p00185.

[108] A. W. Herbert, C. P. Jackson, and D. A. Lever. "Coupled groundwater flow and solute transport with fluid density strongly dependent upon concentration". In: Water Resources Research 24.10 (1988), pp. 1781-1795. DOI: 10.1029 / WR024i010p01781.

[109] R. C. Weast. CRC Handbook of Chemistry and Physics. CRC Press Inc.,U.S., 1975.

[110] M. Ernst. "Numerical simulation of polygonal patterns in salt playa". Master's Thesis. Georg-August University Göttingen, 2017. URL: http : / /hdl . handle.net/21.11116/0000-0002-16A7-9.

[111] D. D. Gray and A. Giorgini. "The validity of the boussinesq approximation for liquids and gases". In: International Journal of Heat and Mass Transfer 19.5 (1976), pp. 545-551. DOI: https ://doi .org/10 .1016/0017-9310(76)90168$\mathrm{X}$.

[112] D. R. Hewitt, J. A. Neufeld, and J. R. Lister. "Stability of columnar convection in a porous medium". In: Journal of Fluid Mechanics 737 (2013), pp. 205-231. DOI: $10.1017 / \mathrm{jfm} .2013 .559$.

[113] J. J. Hidalgo et al. "Scaling of Convective Mixing in Porous Media". In: Physical Review Letters 109.26 (2012). DOI: 10.1103/physrevlett.109. 264503.

[114] C. Güler and G. D. Thyne. "Hydrologic and geologic factors controlling surface and groundwater chemistry in Indian Wells-Owens Valley area, southeastern California, USA". In: Journal of Hydrology 285.1 (2004), pp. 177-198. DOI: https://doi.org/10.1016/j.jhydrol.2003.08.019. 
[115] M. Ruith and E. Beiburg. "Miscible rectilinear displacements with gravity override. Part 1. Homogeneous porous medium". In: Journal of Fluid Mechanics 420 (2000), pp. 225-257. DOI: 10.1017/s0022112000001543.

[116] A. Riaz and E. Meiburg. "Three-dimensional miscible displacement simulations in homogeneous porous media with gravity override". In: Journal of Fluid Mechanics 494 (2003), pp. 95-117. DOI: 10.1017/s0022112003005974.

[117] C.-Y. Chen. Topics in miscible porous media flows. University of Southern California, 1998.

[118] Y. Xie et al. "Effect of transient solute loading on free convection in porous media". In: Water Resources Research 46.11 (2010). DOI: 10 .1029/2010WR009314.

[119] G. de Josselin de Jong. "Singularity distributions for the analysis of multiplefluid flow through porous media". In: Journal of Geophysical Research 65.11 (1960), pp. 3739-3758. DOI: 10.1029/jz065i011p03739.

[120] C.-Y. CHEN and E. MEIBURG. "Miscible porous media displacements in the quarter five-spot configuration. Part 2. Effect of heterogeneities". In: Journal of Fluid Mechanics 371 (1998), pp. 269-299. DOI: 10 .1017/S0022112098002201.

[121] S. K. Lele. "Compact finite difference schemes with spectral-like resolution". In: Journal of Computational Physics 103.1 (1992), pp. 16-42. DOI: 10 . 1016 / 0021-9991 (92) 90324-r.

[122] J. G. Tyler. "Analysis and implementation of high-order compact finite difference schemes". In: (2007). URL: http://hdl . lib. byu . edu/1877/etd2177.

[123] C. Canuto et al. Spectral Methods in Fluid Dynamics. Springer Berlin Heidelberg, 1988. DOI: 10.1007/978-3-642-84108-8.

[124] W. H. Press et al. Numerical Recipes 3rd Edition: The Art of Scientific Computing. Cambridge University Press, 2007.

[125] A. C. Slim and T. S. Ramakrishnan. "Onset and cessation of time-dependent, dissolution-driven convection in porous media". In: Physics of Fluids 22.12 (2010), p. 124103. DOI: $10.1063 / 1.3528009$.

[126] H. S. Hele-Shaw. "The Flow of Water". In: Nature 58.1489 (1898), pp. 34-36. DOI: $10.1038 / 058034 \mathrm{a} 0$.

[127] S. Backhaus, K. Turitsyn, and R. E. Ecke. "Convective Instability and Mass Transport of Diffusion Layers in a Hele-Shaw Geometry". In: Physical Review Letters 106.10 (2011). DOI: 10.1103/physrevlett.106.104501.

[128] J. S. Turner. "Double-Diffusive Phenomena". In: Annual Review of Fluid Mechanics 6.1 (1974), pp. 37-54. DOI: 10.1146/annurev.f1.06.010174.000345.

[129] C. A. Cooper, R. J. Glass, and S. W. Tyler. "Effect of buoyancy ratio on the development of double-diffusive finger convection in a Hele-Shaw Cell". In: Water Resources Research 37.9 (2001), pp. 2323-2332. DOI: 10 . 1029/2001WR000343. 
[130] R. A. Wooding. "Variable-density saturated flow with modified Darcy's law: The salt lake problem and circulation". In: Water Resources Research 43.2 (2007). DOI: $10.1029 / 2005 \mathrm{wr} 004377$.

[131] C. Simmons, M. Pierini, and J. Hutson. "Laboratory Investigation of VariableDensity Flow and Solute Transport in Unsaturated-Saturated Porous Media". In: Transport in Porous Media 47.2 (2002), pp. 215-244. DOI: 10.1023/ A : 1015568724369.

[132] J. A. Neufeld et al. "Convective dissolution of carbon dioxide in saline aquifers". In: Geophysical Research Letters 37.22 (2010). DOI: 10 .1029/2010gl044728.

[133] C. Thomas et al. "Convective dissolution of $\mathrm{CO}_{2}$ in reactive alkaline solutions: Active role of spectator ions". In: International Journal of Greenhouse Gas Control 53 (2016), pp. 230-242. DOI: https : // doi .org/10 . 1016/ j . ijggc . 2016.07 .034$.

[134] S. Mojtaba et al. "Experimental study of density-driven convection effects on $\mathrm{CO}_{2}$ dissolution rate in formation water for geological storage". In: Journal of Natural Gas Science and Engineering 21 (2014), pp. 600-607. DOI: https : //doi . org/10.1016/j.jngse.2014.09.020.

[135] T. Birte. "Experiments for the investigation of convection of salt water in porous media". Bachelor's Thesis. Georg-August University Göttingen, 2016.

[136] "Wentworth scale". In: Encyclopedia of Soil Science. Springer Netherlands, pp. 830830. DOI: $10.1007 / 978-1-4020-3995-9 \_634$.

[137] J.Sauter. “Die Grössenbestimmung der in Gemischnebeln von Verbrennungskraftmaschinen vorhandenen Brennstoffteilchen". In: VDI-Forschungsheft 279 (1928).

[138] D. Wang and L. S. Fan. "2 - Particle characterization and behavior relevant to fluidized bed combustion and gasification systems". In: Fluidized Bed Technologies for Near-Zero Emission Combustion and Gasification. Woodhead Publishing, 2013, pp. 42-76. DOI: https ://doi .org/10.1533/9780857098801.1. 42.

[139] J. Kozeny. “Ueber Kapillare Leitung der Wasser in Boden”. In: Royal Academy of Science, Vienna, Proc. Class I 136 (1927), pp. 271-306.

[140] P. Carman. "Fluid flow through granular beds". In: Chemical Engineering Research and Design 75 (1997), S32-S48. DOI: 10. 1016/s0263-8762(97)80003-2.

[141] X. Garcia et al. "Numerical study of the effects of particle shape and polydispersity on permeability". In: Physical Review E 80.2 (2009). DOI: 10 .1103/ physreve.80.021304.

[142] M. A. Knackstedt and X. Zhang. "Direct evaluation of length scales and structural parameters associated with flow in porous media". In: Physical Review E 50 (1994), pp. 2134-2138. DOI: 10.1103/PhysRevE.50.2134. 
[143] S. Torquato. Random Heterogeneous Materials. Springer New York, 2002. DOI: 10.1007/978-1-4757-6355-3.

[144] A. Revil and L. M. Cathles. "Permeability of shaly sands". In: Water Resources Research 35.3 (1999), pp. 651-662. DOI: 10.1029/98WR02700.

[145] D. Marion et al. "Compressional velocity and porosity in sand-clay mixtures". In: Geophysics 57.4 (1992), pp. 554-563. DOI: 10.1190/1.1443269.

[146] D. C. Beard and P. Weyl. "Influence of Texture on Porosity and Permeability of Unconsolidated Sand". In: The American Association of Petroleum Geologists Bulletin 57 (1973), pp. 349-369.

[147] V. Loodts et al. "Control of Convective Dissolution by Chemical Reactions: General Classification and Application toCO2Dissolution in Reactive Aqueous Solutions". In: Physical Review Letters 113.11 (2014). DOI: 10.1103/physrevlett . 113.114501.

[148] I. Friedman, G. I. Smith, and K. G. Hardcastle. "Studies of quaternary saline lakes-II. Isotopic and compositional changes during desiccation of the brines in Owens Lake, California, 1969-1971". In: Geochimica et Cosmochimica Acta 40.5 (1976), pp. 501-511. DOI: https : / / doi .org/10 . 1016/0016 - 7037 (76) 90218-0.

[149] J. Ryu et al. "Sulfur biogeochemistry and isotopic fractionation in shallow groundwater and sediments of Owens Dry Lake, California". In: Chemical Geology 229.4 (2006), pp. 257-272. DOI: https : //doi . org/10 . 1016/j . chemgeo . 2005.11 .001$.

[150] A. Binley, S. Henry-Poulter, and B. Shaw. "Examination of Solute Transport in an Undisturbed Soil Column Using Electrical Resistance Tomography". In: Water Resources Research 32.4 (1996), pp. 763-769. DOI: 10.1029/95WR02995.

[151] D. J. LaBrecque et al. "Small-Scale Electrical Resistivity Tomography of Wet Fractured Rocks". In: Groundwater 42.1 (2004), pp. 111-118. DOI: 10.1111/j . 1745-6584.2004.tb02456.x.

[152] P. Bauer et al. "Geoelectrical imaging of groundwater salinization in the Okavango Delta, Botswana". In: Journal of Applied Geophysics 60.2 (2006), pp. 126141. DOI: https://doi .org/10.1016/j . jappgeo. 2006.01.003.

[153] C. Power et al. "Improved time-lapse electrical resistivity tomography monitoring of dense non-aqueous phase liquids with surface-to-horizontal borehole arrays". In: Journal of Applied Geophysics 112 (2015), pp. 1-13. DOI: https : //doi.org/10.1016/j.jappgeo.2014.10.022.

[154] J. M. Nield et al. "Estimating aerodynamic roughness over complex surface terrain". In: Journal of Geophysical Research: Atmospheres 118.23 (2013), pp. 1294812961. DOI: 10.1002/2013JD020632. 
[155] Y. Goda. Random Seas and Design of Maritime Structures. World Scientific, 2010. DOI: $10.1142 / 7425$.

[156] J. S. Johnson et al. "Effects of firing temperature on the fate of naturally occurring organic matter in clays". In: Journal of Archaeological Science 15.4 (1988), pp. 403-414. DOI: 10.1016/0305-4403(88)90038-6.

[157] A. B. N. and E. Peter. "Estimation of evaporation from the Dead Sea". In: Hydrological Processes 13.17 (1999), pp. 2743-2750. DOI: 10.1002/(SICI) 10991085 (19991215) 13:17<2743: : AID-HYP845>3 . 0 . CO;2-U.

[158] S. M. Shokri-Kuehni et al. "Impact of type of salt and ambient conditions on saline water evaporation from porous media". In: Advances in Water Resources 105 (2017), pp. 154-161. DOI: https : //doi .org/10.1016/j . advwatres . 2017. 05.004.

[159] R. P. Sharp and A. F. Glazner. Geology Underfoot in Death Valley and Owens Valley. Mountain Press, 1997.

[160] G. Wilkerson et al. Roadside Geology and Mining History: Owens Valley and Mono Basin. Buena Vista Museum of Natural History, 2007.

[161] C. B. Hunt et al. Hydrologic basin, Death Valley, California. 1966. DOI: 10.3133/ pp494b.

[162] H. C. Robertson. "Sedimentology and evaporite genesis in a Holocene continentalsabkha playa basin-Bristol Dry Lake, California". In: Sedimentology 29.2 (1982), pp. 239-253. DOI: 10.1111/j.1365-3091.1982.tb01721.x.

[163] A. Pellew and F. Southwekk. "On maintained convective motion in a fluid heated from below". In: Proceedings of the Royal Society of London A 176.966 (1940), pp. 312-343. DOI: 10.1098/rspa.1940.0092.

[164] R. Charlton. Fundamentals of Fluvial Geomorphology. Routledge, 2007.

[165] P. C. Hohenberg and B. I. Halperin. "Theory of dynamic critical phenomena". In: Reviews of Modern Physics 49.3 (1977), pp. 435-479. DOI: 10 . 1103 / revmodphys. 49.435 .

[166] L. Onsager. "Reciprocal Relations in Irreversible Processes. I." In: Physical Review 37.4 (1931), pp. 405-426. DOI: 10.1103/physrev .37. 405.

[167] O. Annunziata et al. "Mutual Diffusion Coefficients and Densities at 298.15 $\mathrm{K}$ of Aqueous Mixtures of $\mathrm{NaCl}$ and $\mathrm{Na}_{2} \mathrm{SO}_{4}$ for Six Different Solute Fractions at a Total Molarity of $1.500 \mathrm{~mol} \cdot \mathrm{dm}^{-3}$ and of Aqueous $\mathrm{Na}_{2} \mathrm{SO}_{4}{ }^{\prime \prime}$. In: Journal of Chemical \& Engineering Data 45.5 (2000), pp. 936-945. DOI: 10.1021/ je000134s.

[168] H. L. Weissberg. "Effective Diffusion Coefficient in Porous Media". In: Journal of Applied Physics 34.9 (1963), pp. 2636-2639. DOI: 10.1063/1.1729783. 
[169] B. P. Boudreau. "The diffusive tortuosity of fine-grained unlithified sediments". In: Geochimica et Cosmochimica Acta 60.16 (1996), pp.3139-3142. DOI: 10.1016/ 0016-7037 (96) 00158-5.

[170] B. P. Boudreau. Diagenetic Models and Their Implementation: Modelling Transport and Reactions in Aquatic Sediments. Springer, 2011.

[171] L. Sachs. Angewandte Statistik. Springer Berlin Heidelberg, 2002. DOI: 10 . 1007/978-3-662-05745-2.

[172] G. Kocurek, M. Townsley, and K. E. Yeh. "Dune and Dune-Field Development on Padre Island, Texas, with Implications for Interdune Deposition and Water-Table-Controlled Accumulation". In: SEPM Journal of Sedimentary Research Vol. 62 (1992). DOI: 10 . 1306/d4267974-2b26-11d7-8648000102c1865d.

[173] B. Hallet, R. Sletten, and K. Whilden. "Micro-relief development in polygonal patterned ground in the Dry Valleys of Antarctica". In: Quaternary Research 75.02 (2011), pp. 347-355. DOI: 10.1016/j · yqres . 2010.12.009.

[174] S. M. S. Shokri-Kuehni et al. "New insights into saline water evaporation from porous media: Complex interaction between evaporation rates, precipitation, and surface temperature". In: Geophysical Research Letters 44.11 (2017), pp. 5504-5510. DOI: 10.1002/2017g1073337.

[175] S. Gupta et al. "Paradoxical drying of a fired-clay brick due to salt crystallization". In: Chemical Engineering Science 109 (2014), pp. 204-211. DOI: https : //doi.org/10.1016/j.ces.2014.01.023.

[176] G. Taylor. "Dispersion of soluble matter in solvent flowing slowly through a tube". In: Proceedings of the Royal Society of London A: Mathematical, Physical and Engineering Sciences. Vol. 219. 1137. 1953, pp. 186-203.

[177] R. Aris. "On the Dispersion of a Solute in a Fluid Flowing through a Tube". In: Proceedings of the Royal Society A: Mathematical, Physical and Engineering Sciences 235.1200 (1956), pp. 67-77. DOI: 10.1098/rspa.1956. 0065.

[178] R. N. Horne and F. Rodriguez. "Dispersion in tracer flow in fractured geothermal systems". In: Geophysical Research Letters 10.4 (1983), pp. 289-292. DOI: 10. 1029/g1010i004p00289. URL: https://doi .org/10.1029/g1010i004p00289.

[179] M. Norouzi Rad and N. Shokri. "Nonlinear effects of salt concentrations on evaporation from porous media". In: Geophysical Research Letters 39.4 (2012). DOI: $10.1029 / 2011$ GL050763.

[180] C. J. van Duijn et al. "Stability criteria for the vertical boundary layer formed by throughflow near the surface of a porous medium". In: Environmental Mechanics: Water, Mass and Energy Transfer in the Biosphere. American Geophysical Union, 2002, pp. 155-169. DOI: 10.1029/129gm15. 
[181] A. Riaz et al. "Onset of convection in a gravitationally unstable diffusive boundary layer in porous media". In: Journal of Fluid Mechanics 548 (2006), pp. 87-111. DOI: 10.1017/S0022112005007494.

[182] Y. A. Cengel. Heat Transfer: A Practical Approach. Mcgraw-Hill (Tx), 2002.

[183] J. M. Nield et al. "Evaporative sodium salt crust development and its wind tunnel derived transport dynamics under variable climatic conditions". In: Aeolian Research 23 (2016), pp. 51-62. ISSN: 1875-9637. DOI: https : / / doi . org/10.1016/j.aeolia.2016.09.003.

[184] M. N. Rad et al. "Effects of Grain and Pore Size on Salt Precipitation During Evaporation from Porous Media". In: Transport in Porous Media 110.2 (2015), pp. 281-294.

[185] M. Bergstad et al. "The influence of $\mathrm{NaCl}$ concentration on salt precipitation in heterogeneous porous media". In: Water Resources Research 53.2 (2017), pp. 1702-1712. DOI: 10.1002/2016wr020060.

[186] M. Bergstad et al. "Evaporation Dynamics and $\mathrm{NaCl}$ Precipitation on CapillarityCoupled Heterogeneous Porous Surfaces". In: Water Resources Research 54.6 (2018), pp. 3876-3885. DOI: $10.1029 / 2018 w r 022614$.

[187] D. Bevc and H. F. Morrison. "Boreholetosurface electrical resistivity monitoring of a salt water injection experiment". In: Geophysics 56.6 (1991), pp. 769777. DOI: $10.1190 / 1.1443094$.

[188] C. Palache, H. Berman, and C. Frondel. "Dana's System of Mineralogy. 7. Ed". In: Geologiska Föreningen i Stockholm Förhandlingar 74.2 (1952), pp. 218219. DOI: $10.1080 / 11035895209453366$.

[189] H. E. A. Brand et al. "The thermal expansion and crystal structure of mirabilite $\left(\mathrm{Na}_{2} \mathrm{SO}_{4} \cdot 10 \mathrm{D}_{2} \mathrm{O}\right)$ from 4.2 to $300 \mathrm{~K}$, determined by time-of-flight neutron powder diffraction". In: Physics and Chemistry of Minerals 36.1 (2008), pp. 2946. DOI: $10.1007 / \mathrm{s} 00269-008-0256-0$.

[190] A. Adamson. A Textbook of Physical Chemistry. Academic Press, 2012.

[191] M. Bestehorn and H. Haken. "A calculation of transient solutions describing roll and hexagon formation in the convection instability". In: Physics Letters A 99.6-7 (1983), pp. 265-267. DOI: 10 .1016/0375-9601 (83) 90880-0.

[192] J. R. de Bruyn et al. "Apparatus for the study of Rayleigh-Bénard convection in gases under pressure". In: Review of Scientific Instruments 67.6 (1996), pp. 2043-2067. DOI: 10.1063/1.1147511.

[193] M. D. Shattuck et al. "Convection and flow in porous media. Part 1. Visualization by magnetic resonance imaging". In: Journal of Fluid Mechanics 332 (1997), pp. 215-245. DOI: 10.1017/s0022112096003990. 
[194] G. S. Pau et al. "High-resolution simulation and characterization of densitydriven flow in $\mathrm{CO}_{2}$ storage in saline aquifers". In: Advances in Water Resources 33.4 (2010), pp. 443-455. DOI: https : / / doi . org/10 . 1016/ j . advwatres . 2010.01 .009$.

[195] R. M. Clever and F. H. Busse. "Hexagonal convection cells under conditions of vertical symmetry". In: Physical Review E 53.3 (1996), R2037-R2040. DOI: 10.1103/physreve.53.r2037.

[196] J. Lasser and E. Katifori. "NET: a new framework for the vectorization and examination of network data". In: Source Code for Biology and Medicine 12.1 (2017). DOI: $10.1186 /$ s13029-017-0064-3.

[197] G. Einsele. Sedimentary Basins. Springer Berlin Heidelberg, 1992. DOI: 10 . 1007/978-3-642-77055-5. 